

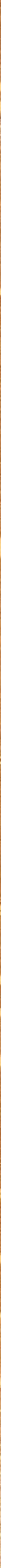









\section{VOYAGE}

\section{AUTOUR DU MONDE}

SUR I.A FREGATE

LA VÉNUS

ZOOLOGIE 
PARIS - INPIIUERIE J, CLAIE

PUE EAINT-BESOIT. 7. 


\section{VOYAGE}

\section{AUTOUR DU MONDE}

SIK LA HHEGATE.

\section{LA VÉNUS}

(1) $111 \mathrm{~A} \times \mathrm{N}) \mathrm{E}$

\section{PAR AREL DU PETIT-TEOUARS}

Cappitame de vaisseau, Commandeur de la Legion d'honmeu

$$
\text { ZOOLOGIE }
$$

WAMMIELES, OISEIUX, REPTIES ET DOISSONS

\section{3}

\section{PARIS}

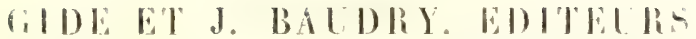

I: IE THXALAHED

$18: 3$ 


$$
\text { Fon is : is: }
$$




\section{MAMMUFERS.}

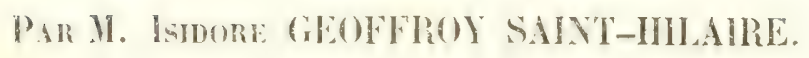

La mammalogie, ordinairement si négligée dans les voyages de circumnavigation, doit à l'expédition de la Vénus plusieurs acquuisitions intéressantes. M. le contre-amiral Du Petit-Thouars, au milieu des devoirs et des soins multipliés du commandement; MI. le docteur Néboux, malgré ses occupations de tous les instants comme chirurgien-major de la frégate, ont su trouver, dans leur zèle éclairé pour la science, les moyens d'enrichir les collections de la Vénus de divers représentants mammalogiques des contrées successivement visitées par l'expédition. L'un et l'autre ont recueilli un assez grand nombre d'éléments et de matériaux, précieux pour l'histoire spéciale de la première classe du règne animal, en nème temps que pour la géographie zoologique.

Invité par M. le contre-amiral Du Petit-Thouars à me charger du soin de mettre en oeuvre ces élćments et ces matériaux, je n'ai pas hésité à accepter ce deZoologie. 
voir. Afin de m'en acquitter d'une manière plus digne de l'importance de l'expielition et plus fructuense pour la science, j'ai cru devoir suivre de nouveau ici le plan que je m’étais tracé dans plusieurs publications analogues ${ }^{1}$, et dont l'expérience m’a démontré de plus en plus les avantages. In dicrivant les espèces nouvelles ou imparfaitement connues qui ont été rapportées par la Ténus, je les rapprocherai de leurs congénères, soit déjic connus, soit récemment découverts et encore inćdits; je considérerai les uns et les autres sous un point de vue comparatif; et, par--là même, je chercherai à faire nettement ressortir ce que les espèces établies dans ce travail ont en elles de spécial et de caractéristique, et ce qu'elles peuvent offrir de neuf el d'intéressant pour la science.

Parfois même, lorsque les familles el les genres auxquels se rapportent ces espèces, n’auront point encore été étudiés d'une manière aussi complèto que permet de le faire l'élat actuel de la science, je présenterai aussi sur eux quelques considérations générales, et je ne croirai pas sortir de mon sujet en donnant l'indication succincte de toutes les espèces congénères anciennement connues ou qu'il m'aura été possible de déterminer par mes propres observations. L'immense avantage des travaux monographiques

1 Dans les parties erpétologique et ichthyologique du grand Ourrage sur l'W F ple, 1827 ; dans la partie mammalogique de IOuragé sur la Moréc, 1833 ; mais surtout dans les parties mammalogiques du Voinge aux Indes Orientales de Bélanger, $1830-$

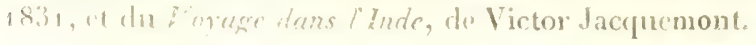


v'est, aujourd'hui, conteste par persome; mais peutêtre la science recevrait-elle un accroissement plus rapide encore, si les naturalistes de chaque pays, principalement ceux qui se trouvent placés près des grankes collections, atoptaient l'usage d'ajouter it la description de's especes nouvelles qu'ils publient, le tableatr des esperes anciennement décrites, dont ils ont par eux-mêmes constaté l'existence. Sil en était ainsi, plusieurs de celles qui restent douteuses encore, seraient bientot delinitivenent ctablies dans la science; et d'autres au contraire ne tarderaient pas à être recomnes pour nominales on ctablies seulement sur de doubles emplois, et à être retranchées du systema. 



\title{
PREMIERE SEGTION.
}

\author{
PRIMATES.
}

Parmi les quatre familles qui composent l'ordre des P'rimates, trois, celles des Lémuridés, des Tarsidés et des Cheiromy dés, hałitent exclusivement des rígions que n'a point visitces l'expédition, et je ne m'arrèterai pas ici sur clles. La famille des Singes compte au contraire des représentants plus ou moins nombreux sur plusieurs points de l'itinćraire de la $V$ énus, et notamment au cap de Bonne-Espérance et au Brésil. Parmi les Singes de l'Afrique australe, je décrirai une espèce lepuis longtemps connue, mais non distinguće par les meilleurs auteurs eux-mêmes, le Cercopithègue Delalande, dont jéclaircirai et compléterai la détermination par un synopsis des espèces congénères. De mème, à l'occasion de quelques Singes du Brésil, je chercherai ì ćclaircir l'histoire, encore fort embrouillée, des Singes anćricains, à queue faiblement prenante, et particulièrement à montrer que les Saïmiris, réunis par presque tous les auteurs aux Callitriches, constituent en réalité l'un des genres les plus distincts et les plus remarquables de la grande famille des Singes. 


\section{PREMIERE PARTIE.}

Descriprox de CERCOPTTHEQUE DELALANDE, Cercopithecus Lalandii, Is. Geoff.; ex Synorsis du gevre Cercopishìoue.

\$1. - Remarques préliminaires sur les Singes du Cap de Bonne-Espérance.

T'andis qu'en Amérique, les Singes, si abondamment répandus dans la région centrale, disparaissent au sud et au nord à une distance très-considérable des extrémités de ce continent, l'Afrique, que l'équateur coupe exactement par son milieu, et dont les extrémilés au sud et au nor l ne dépassent les tropiques que d'un perit nombre de degrés, possède, dans toute son étendue, des représentants de la grande famille des Singes. Il existe toutefois une très-grancle différence entre te nombre des especes qui peuplent l'Afrique contrale et le nombre de celles qui habient les deux extrémités de l'Afrique. Ainsi, dans l'Afrique centrale, les contrées équatoriales, telles que la Guinće et le Congo, ont foumi déjà et fournissent charue jour une mullitude d'respèces, appartenant aux sept genres Troglodyle, Colobe, Miopithèque, Cercopithèque, Cercocèbe ou Mangabey, 'Théropithèque el Cynocéphale : vers les tropiques au contraire, plusieurs de ces genres sont deja sans représentants, ou ne sont plus représentés que par un très-petit nombre d'es- 
pèces; et tout-à-fait vers l'extrémité, au-delà du trentième degré, nous ne trouvons plus au nord, en Barbarje, au sud, au cap de Bonne-Espérance, qu'un très-petit nombre de Singes, tous dépourvus de ces couleurs vives et de ces développements du pelage qui rendent si remarquables presque toutes les espèces de la région équatoriale de l'Afrique.

Au cap de Bonne-Espérance, en particulier, on ne comnait que deux espèces, un Cynocéphale et un Cercopithèque ${ }^{1}$.

La première est le Cynocephalus porcarius des auteurs, le Choak-Kama des Hottentots; espèce généralement connue sous le nom de Chacma, et que caractérisent suffisarmment sa grande taille et son pelage d'un brun-verdàtre, sans crinière. Tous les voyageurs qui ont exploré l'Afrique australe, particulièrement Kolbe, dont les récits, presque toujours mêlés de fables, ne peuvent d'ailleurs être acceptés qu’en partie, Sparman, Levaiban, Thumberg ${ }^{2}$, Delalande ,

a Sparman mentionne, il est vrai, dans son Voyage au Cap dc Bonne-Espérance (traduct. de Letourneur, t. 1, p. 306), des Singes qui sembleraient n'être ni le Cynocéphale ni le Cercopitheque que nous connaissons; car il les dit noirs comme le charbon, et de la grosscur à peu près d'un chat ordinaire. Mais Sparrman n'avait vu ces Singes que de loin, et la courte indication qu'il donne, n'est d'aucune valeur.

"Sous le nom inexact de Simia sphynx. Voyez les Mém. de l'Acad. imp. de Saint-Pélersbouror, t. I11, p. 301, 1811.

- Les faits très-intéressants qu'a recueillis Delalande sur le Cynocephalus porcartus ont été publiés, d'après les notes prises par lui sur les lieux, par Desmoulins, dans l'article Cynocéphale du Dictionnaire classique d'histoire naturelle, l. V, p. 257 et 260. 
Burchell ', nous ont transmis des renseignements plus ou moins nombreux sur cette espèce qui, d'ailleurs, a vécu plusieurs fois en Europe dans les ménageries, dont il existe de nombreux individus dans toutes les grandes collections, et que l'on peut considérer aujourd"hui comme bien déterminée et bien connue.

Il en serait de même du Cercopithèque de l'Afrique australe, si l'on devait en croire les auteurs. Tous s'accordent ì désigner comme habitant le cap de Bonne-Espérance avec le Cynocephalus porcarius, l'espèce que M. Frédéric Cuvier, dans la première édition de son ouvrage sur les Mammifères, a décrite et établie en 1821 sous le nom de Vervel, Simia pygerythra; puis décrite de nouveau, quelques années plus tard, dans la seconde édition du même ouvrage, sous le nom de Cercopithecus prgerythrus. Les mêmes indications ${ }^{2}$ se trouvent, à l'égard de cette prétendue espèce du Cap, dans tous les ouvrages généraux sur les mammiferes, tels que la Manmalogie de M. Desmarest ${ }^{3}$, le Synopsis Mammalium de J.-B.

1 Sous le nom de Cercopithecus ursinus dans plusieurs passages de ses Travels in the interior of southern Africa.

2 Seulement l'espèce est nommée tantôt Simia pyegerythra ou Cercopithecus pygerythrus, tantôt $S$. erythropyga (nom employé par G. Cuvier, qui l'attribue à tort à Fr. Cuvies), tantôt Cercopithecus ou Cercoccbus pygerythraus, ou S. pygeryethaa. C'est Desmarest qui a introduit cette dernière modification, afin de rectifier un nom qu'il croyait irrégulièrement formé. Il se trompait à cet égard: on dit également Éovopós et Épupä́os. Il n'y a donc aucun motif pour ne pas adopter le nom le plus ancien.

3 Dans le Supplément. L'espèce n'était pas comnue lorsque M. Desmarest a fait paraître la première partie de son ouvrage. 
Fischer, le Physical history of Man and Quadrumana de M. Limneus Nartin, le Hanuel de Hammalogie et le Species des Mammifere's bimanes et quadrumanes de M. I.esson, et un grand nombre d'autres.

On les trouve également reproduites dans les ouvrages spéciaux sur la Faume d'Afrique ou sur les Mammifères du cap de Bonne-Espérance.

Ainsi, le docteur A. Smith, dans son African zoo$\log )^{1}$, place à la suite des Cercopithecus sabceus et $C$. viridis (qu'il nomme génériquement Cercocebus),

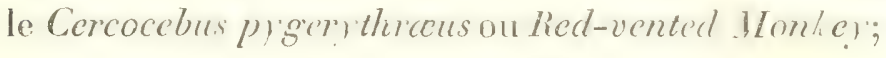
et il ajonte: "Inhabits south Africa, and troops... "are often seen in woods and thickets, at no great " distance from Cape Town."

De même, dans un ouvrage plus spécial encore, et composé avec beaucoup de soin, Enumeratio Mammalium Capensium², Smuts, selon l'exemple des auteurs qui l'ont précédé, place à la tête des mammiferes de l'Afrique australe le Cercopithecus pygerythrceus, dont il donne ainsi l'habitat: "Degunt " in plagis magis syluestribus, nec prope urbem " principem Promontorï occurrunt."

Si l'on ne savait avec quelle facilité une erreur une fois introduite dans la science, y prend peu à peu racine, et finit par y être universellement admise, j'oserais à peine dire, après tant d'assertions positives

' Insérée dans The south African quarterly' journal, t. II, Cap? de Boune-Esperance, 1835.

- In-4. Leyde, $183 \%$. 
inances de zoologistes distingués ondinaiternent dignes de toute confiance, que la patrie du Cercopithecus p) gerythrus reste aujourd'hui encore tout-à-fait inconnue; et même si je puis affirmer quelque chose ì son égard, c'est que cette patrie n'est pas le cap de Bonne-Espérance. Il est hors de toute vraisemblance que le C.pygerythrus, s'il avait l'habilat qu'on lui attribue, eût pu échapper aux recherches de Delalande, de ses neveux et si dignes continuateurs, MM. Verreaux, et de tant d'autres. Il est d'ailleurs à remarquer que la presque identité du pelage du $C$. pygerythrus avec celui du C. Sabceus qui habite le Sénégal, en celui du C. griseo-viridis, qui habite la Nubie et le sud de l'Egypte, indique à elle seule une analogie très-grande dans la zone d'habitat de ces trois espèces; considération qui, au premier aspect, semble de per d'importance, mais qui empruntera quelque valeur de l'examen que je vais faire tout-àl'heure de l'ćtat du pelage chez le véritable Cercopithèque du Cap, le Cercopithèque Delalande.

Les détails dans lesquels je vais entrer feront voir aussi que toutes les assertions inexactes que j'ai dû rappeler plus hatil, ont leur source dans la confusion qui a ćté faite, d'abord par M. Fr. Cuvier, puis, à son exemple, par les autres zoologistes, entre le $C$. pjgerythrus et le C. Lalandii; espèce voisine, mais distincte du C. pygerythrus, et qui m'est aujourd'hui connue par une belle série d'individus des deux sexes et de tout îge. Je me plais, du reste, à reconmaitre que l'erreur que je relève ici, était à peu près inevilit- 
ble à l'époque où elle a été commise, et lorsqu'on ne possedait encore quime trés-faible partis: des matériaux dont je puis aujourd'hui disposer.

\section{SII.-Historique et synonymie.}

L'histoire ei la synonymie du Cereopithèque Dela-

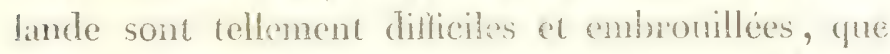
quelques remarques explicatives me paraissent devont nécessairement précóder la deseription de l'espéce.

La Cereopitheque Delalamle a dit successivement wonsidere comme spécifiguenent le mème que le C: sabcus des auteurs (Simia saboea, Lrvo), comme identique avec le $C$. py'gerythrus de M. Fr. Cuvier, et comme constituant une espèce distincte, principalement caractérisée par la petitesse de sa taille. Ces trois opinions sont également erronées.

Le C. pygerythrus est, en réalité, une espèce exactement de même taille que la plupart de ses congénères, et se distinguant par des différences de pelage et non de dimensions.

C'est Thunberg qui, ayant l'un des premiers observé de Cercopihique Delalande, l'a considere' comme ne différant pas du C. sabceus. Cette erreur était naturelle à une époque où l'on n'avait point encore introduit, dans la détermination des espèces, cette précision, parfois un pen minutieuse, mais indispensable, yui caractirise la zoologie actuelle. Il suftisait pres-

1 Loc. cul., 1811. 
que alors que le Cercopithèque nouvellement observe eût les formes et les proportions du C. sabceus, et le pelage généralement verdâtre, pour qu'on crût reconnaitre en lui celte espèce, si souvent désignée sous le nom de Callitriche ou Singe-vert. Aujourd'hui il serait superflu d'insister sur les caractères differentiels du $C$. sabceus, espèce aussi commune qu'elle est facile à reconnaître, et par suite depuis longtemps et complétement déterminée.

En rectifiant l'erreur commise par 'Thunberg, les zoologistes récents en ont eux-mêmes laissé échapper une autre. Dans les immenses collections faites dans l'Afrique australe par Delalande, se trouvaient quelques individus de l'espéce précédenment observée par Thunberg, et avant lui par Levaillant; mais ces individus étaient tous fort jeunes. Lor's de l'arrivée en France des collections de Delalande, MI. Fr. Cuvier crut trouver dans ces jeunes Singes le premier âge d'un Cercopithèque qui vivait alors à la ménagerie du Muséum, et dont ce savant zoologiste ${ }^{1}$ a fait le type de son $C$. py'gerythrus. C'était une erreur, mais une erreur à laquelle il était alors difficile d'échapper : les affinités qui existent entre le $C$. pygerythrus et l'espèce de Levaillant, de Thumberg et de Delalande, sont tellement intimes, qu'ayant sous les yeux, d'un côté, l'état adulte du premier sans son jeune âge, et, de l'autre, le très-jeme inge de la seconde sans son état adilte, or devait ctre portó it atribues it de simples différence- 
l'áge les diflérences que l'on apercerait mutro le pelage de l'une et celui de l'autre. M. Frédéric Cuvier n'hésita donc pas à les réunir, et c'est par suite de cette confusion, qu'il donna l'Afrique australe pour patrie au C. pygerythrus : espèce n'ayant en réalité pour type qu'un individu, acheté de marchands qui ignoraient entièrement son origine, et dont la véritable patrie reste aujourd'hui même inconnue.

Les auteurs ont généralement suivi M. Frédéric Cuvier, aussi bien pour la caractéristique qu'il assigne au C. p. gery'thrus, que pour la patrie qu'il lui aturibue. Il en est ainsi en particulier de Desmoulins; dans l'article étendu qu'il a publić en 1825 sur le genre Cercopithèque ou Guenon ", ce zoologiste termine la description du C. pygerythrus en disant, d'après Fr. Cuvier, que Delalande a rapporté beaucoup d'individus de cette espèce, du Cap où elle peuple les forêts. Mais, dans un autre passage, le même auteur, sans s'apercevoir qu'il fait un double emploi, cite de nouveau les Singes rapportés par Delalande, et les décrit comme constituant une espèce distincte, ayant le pelage grisàtre, et seulement 9 à 10 pouces (énvirou $\left.0^{\mathrm{m}}, 25\right)$ du bout du museau ì l'origine de la queue. En raison de cette taille prétendue naine, Desmoulins donne à l'espèce le nom, d'ailleurs très-irrégulièrement formé, de Guenon naine Delalande, $C$. pusillus Delalande. C'est sous ce double nom spécifique, formé contrairement aux règles de la nomenclature

I Dans le Dictionn. class. d'hist. natur., t. VII, p. 560 et suiv. 
linncenne, que l'on trouve l'espece pour la premiere fois introduite dans la science ${ }^{2}$ comme distincte. La place que lui assigne l'auteur, n'est pas moins singulière que son nom: le C. pusillus Delalande, décrit comme ayant le pelage d'un gris-cendré, compose, avec le $C$. ruber ou Patas, dont le pelage est rouge, et le C. Tatibarbatus ${ }^{2}$, dont le pelage, dit Desmoulins, est noir à l'état adulte, et roux dans le jeune âge, la section des Singes-verts; section dont ne font partie, au contraire, ni le C. snbaus ou Singe-vert des auteurs, ni les C. cynosurus, $C$. griseo-viridis et $C$. pygerythrus, espèces qui ont toutes le pelage vert.

Toutes ces contradictions avaient déjà fixé mon attention, et j'avais acquis la certitude que Desmoulins avait pris de très-jeunes sujets pour des adultes, lorsque, dans divers envois, et d'abord dans la grande

- Dans ses Reisen in südlichen Africa, dont la publication remonte à 1811 , l’un des zoologistes les plus distingués de l'Allemagne, M. Lichtenstein, paraît, il est vrai, avoir désigné cette espèce sous le nom de $C$. glaucus. Mais ce nom n'est accompagné ni d'une description, ni même d'une caractéristique sommaire; et c'est pourquoi cette indication a été considérée comme non avenue par tous les auteurs. Voici le passage tout entier de M. Lichtenstein ( t. I, p. 507); passage que l'on a négligé jusqu'à présent de citer: "In den Gebiischen durch welche dann und wamn die a Pieise ging, fand man of eine grosse Menge Affen vou der lang* geschwänzten grïnen Art, die dieser Gegend des südlichen "Africa eigen ist. Einzelue der Colonisten besassen eine besona dere Geschiklichkeit darin, diesen niedlichen Thieren behende, * auf die Bäume nach zukletterm, und sie einzufangen.»

a Ce dernier n'est, d'ailleurs, pas un véritable Cercopithèque, mais un Semnopithèque. 
collection rapportée du Cap par MM. Verreaux, je irouvai le $C$. pusillus Delalande, avec tous les caxactères du pelage que je lui connaissajs, mais avec la taille des autres espèces à pelage vert ou verdàtre. Dès-lors il devenait évident que les jeunes Cercopithèques de M. Delalande avaient été à tort rapportés par M. Frédéric Cuvier, et d'après lui, par tous les auteurs, M. Desmoulins excepté, au C. pJ'gerythrus, et qu'ils doivent être considérés, en rectifiant la détermination de celui-ci, comme appartenant à une espèce distincte, plus voisine d'ailleurs qu'aucune autre du C.pygerythrus.

En rectifiant la détermination de M. Desmonlins et la place qu'il assignait à celte espèce, j’ai cru devoir aussi rectifier, mais non abandonner, le nom qu'il lui donnait; nom qui rappelle l'un des plus beaux voyages zoologiques qui aient jamais été exécutés. J'ai donc proposé, d'abord dans mes cour's, puis dans l'article C'ercopithicque du Dictionnaire d'Histoire Nulurelle, de domer a l'espece le nom de Cereopithèque Delalande, $C$. Lalandii.

\section{\$III.-Description.}

Le Cercopitheque Delalaude appartient a la section dont le C. sabceus est le type, section que caractérisent un museau un peu plus allongé que chez les C. petaurista, C. ceplitus et les autres espèces voisines, des formes généralement moins sveltes que chez celles-ci, et un pelage plus ou moins verdâtre. 
Le caractère par lequel on peut le distinguer dès le premier aspect de ses congénères, est la teinte générale du pelage qui n'est pas vert, même sum la tête et sur le dos, mais d'un gris-verdàtre.

Cing couleurs, dont la distribution est caractéristique, se remarquent dans le pelage de cette espèce : le gris-verdatre, le gris-pur, le blanc sale, le noir et le roux.

Le gris-verdàtre occupe le dessus de la tête, du cou, du dos junquái quelques centimètres de l’origine de la queue, les épaules et les flancs. Dans toutes ces parties, il existe deux sortes de poils : des poils laineux, longs et abondants, d'un gris clair à la base, d'un gris foncé dans le reste de leur étenclue; des poils soyeux beaucoup plus longs encove (de 3 à 5 centimètres), colorés, par anneaux d'une assez grande étendue, de noir et de jaune-verdâtre. De là résulte une teinte générale d'un gris sale, légèrement verdàtre, tiqueté de blanchâtre.

La face externe de l'avant-bras, de la cuisse et de la jambe, la croupe et les trois premiers quarts environ de la queue, sont d'un gris tiqueté. La longueur des poils est moindre dans ces parties, mais leur mode de coloration est le même; seulement les poils sont annelés de noir et d'une couleur blanchàtre à peine teintée de jaune.

Le blanc sale occupe la face interne des membres, le dessous du corps et la partie inférieure et latérale de la tête. De plus, il existe un assez large bandeau de même couleur ì la partie antérieure du front, im- 
médiatement derrière les sourcils. Ce bandeau, composé de poils presque ras, se confond à ses deux extrémités avec le blanc de la région latérale de la tête, où lespoils, dirigés en arrière, sont au contraire tres-longs.

Le bandeau blanc frontal et les poils blanes des parties inférieures et latérales de la tête, encadrent la face et le menton qui sont, au contraire, noirs. Les quatre mains et le dernier quart de la queue sont aussi de cette dernière couleur.

Enfin, le roux forme une tache très-remarquable autour de l'anus; tache qui s'étend aussi sous la base de la queue. C'est l'existence d'une tache rousse anale, ubserve d'abord par N. Frédéric Cuvier, chez le Vervet, c'est ce caractère considéré par lui comme propre à ce Singe (d'où le nom de $C$. pygerythrus), qui ont entraîné ce célèbre zoologiste à confondre avec le Vervet, l'espèce dont je viens de décrire les couleurs. J'ai recomnu depuis que, non-seulement la tache analo existe dans deux espèces d'álleurs très-distinctes, mais qu'elle est représentée par quelques poils roux, en d'autres termes, qu'elle existe aussi, mais beau. coup plus petite et moins distincte, dans deux autres espèces, le $C$. cynosurus ou Malbrouck des auteurs, et le C.rufo-viridis.

Le mâle, d'après les renseignements que j'ai recueillis, aurait le scrotum d'un bleu-verdàtre. On sai par les observations de M. Frédéric Cuvier, que cette partie est verdâtre chez le $C$. sabceus, vert-de-gris chez les $C$. griseo-viridis et C. pygerythrus, d'un bleu lapis chez le C. cynosurus. 
Les dimensions prises, ainsi que les caractères précédents, sur l'adulte, sont les suivantes:

Longueur du bout du museau à l'origine de la queue. 0,45

Longueur de la quene. . . . . . . . . . 0,55

J'ai pu comparer aux individus adultes plusieurs autres sujets de différents âges. Les différences de pelage qu'ils m'ont présentées, sont relatives, les unes aux mains, que j'ai trouvées tantôt grisâtres, tantôt noiràtres, mais non noires; les autres à la couleur générale, qui, chez les jeunes sujets on bon état ${ }^{1}$, est plus lavée de vert, et se rapproche beaucoup de la conleur du Macaque ordinaire.

Cette espèce, dont j'ai déjà donné sommairement les caractères dans le Dictionnaire universel d'histoire nalurelle ${ }^{2}$, mais qui n'avait encore été ni décrite en détail, ni figurée, habite exclusivement l'Afrique australe. C'est à MM. Verreaux que l'on doit le plus grand nombre des individus existant au Musée de Paris : ils avaient été tués dans la vaste forêt de l'Auteniquoi, qu'ils habitent par bandes de vingt à trente individus.

' Si, au contraire, les jeunes ne sont pas en bon état, si une: partie des poils soyeux a disparu, le pelage paraît de même nuance que chez les adultes, ou meme encore plus gris, parce que l's poils laineux deviennent plus ou moins apparents.

2 Article Cercopihéque. Voyez t. III, p. 305, 1842.-Voyez aussi un mémoire qui paraîra prochainement dans les Archives du Muséum d'hist. nat., t. II, sous le titre suivant: Description des mammifères nouveaux de la collection du Muséum. Premier memoire. Famille des Singes. 


\section{\$IV. Synopsis du genre Cercopithèque.}

Je ne comprends, dans ce genre, ni les Mangabers qui, d'après divers caractères récemment observés, ne sont pas de véritables Cercopithèques, et pour lesquels il convient de rétablir le genre Cercocebus; ni le Talapoin, dont j'ai fait récemment le type d'un genre nouveau, sous le nom de Miopithecus ${ }^{1}$; ni, à plus forte raison, les Semnopithèques, le Nasique et les Colobes, que personne, depuis vingt ans, ne confond plus, avec les Cercopithèques.

Les travaux par lesquels ont été fondés ces cinq genres, ont enlevé au groupe des Cercopithèques près de trente espèces ${ }^{2}$; et cependant, après toutes

1 Voyez les Comptes rendus hebdom. de l'Acad. des sciences, t. XV, p. 720 et 1037 , et les travaux cités dans lat note précédente.

2Savoir : $1^{\circ}$ deux Cercocèbes : 1. Cencoceudos fuligroosus, Geoff. S.-H.; 2. C. жтиноr, Geoff. S.-H. (Simia cethiops, L.)

$2^{\circ}$ Un Miopithèque : Mropituechus talapoin, Isid. Geoff. $(S$. talapoin, L.)

$3^{\circ}$ Seize Semnopithèques : 1 . Semopitmecus nerzus, Fr. Cuv. (le Douc de Buffon); 2. S. Leucoprymes, Desmar. (Cercop. leucoprgmnus, Otto); 3. S. catibarbatus, Isid. Geoff. (Sim. latibarbata, 'Tem.); 4. S. onscurus, Reid. et Mart. ; 5. S. cucullatus, Isid. Geoff.; 6. S. Dussumeri, Isid. Geoff. ; 7. S. exteluos, Fr. Cuv. (Sim.entellus, Dufresne); 8. S. aunatus, Desmoul. (Cerc. auratus, Geoff. S.-H.); 9. S. cristatus, Mart. (Sim. crislata, Raffl.); 10. S. waunus, F. Cuv. (Sim. maura, Schreb.); 11. S. femoralis, Horsf.; 12. S. Mitratus, Sal, Mull. et Schleg. (Presbytis mitrata, Eschsch.); 13. S. nigrmanus, Isid. (reoff.; 14. S. flayinanus, Is. Geoff.; 15. S. mrialophos, Fr. Cuv. (Sim. 
ces éliminations successives, il en reste encore dans ce genre plus de vingt, toutes habitant, soit le continent africain, soit les îles d'Afrique, telles que les îles du Cap-Vert et Fernando-Po, soit l'Arabie et la Syrie, contrées que les géographes comprennent dans l'Asie, mais qui sont essentiellement, par leurs productions naturelles, de véritables dépendances de l'Afrique.

En raison du nombre des espèces connues de Cercopitheques, et aussi de quelques différences organiques assez marquées entre les premières é les dernières, j'ai cru devoir ${ }^{1}$ diviser les Cercopithèques en

melalophos, Raffl.); 16. S. ruericurdus, Sal. Mull. et Schleg (Dans un travail récent, oì sont décrites plusieur's de ces espèces, la Mammalogie du Voragge de Victor Jacquemont, j'ai montré que: le $S$. albo-cinereus et le $C$. fulvo-griseus de quelques anteurs modernes sont des espèces purement nominales; c'est pourquoi ces.s deux Singes ne figurent point dans ce tableau des Semnopitheques.)

$4^{\circ}$ Un Nasique: Nasalis ingatus, Geolf. S.-H. (Sim. nasica. S. nasalis ou 5 . rostrata des auteurs ).

$5^{\circ}$ Enfin, neufColobes (dont plusieurs restent malheureusement très-mal connus ou même douteux): 1. Conones venenosus, Isid. Geoff. (quej'avais d'abord décrit sous le rom de $5 \mathrm{cmn}$. ve\%. lerosus, et qui est devenu depuis le Scmn. bicolor de M. Wesmael et le Col. leucomeros de M. Ogilby; 2. C. guereza, Rupp.; 3. C. pouxconos, Geoff. S.-H. (Sim. polycomos, Schreb.); 4. C. ursivus, Ogilb. ; 5. C. satanas, Waterh.; 6. C. Fuliginosus, Og.; 7. C. renuugrosus, Geoff. S.-H. (Sim. ferruginea, Sh.); 8. C. pennaxti, Waterh.; 9. C. venus, Van Beneden.

1 Dans un travail resté inédit, mais qui a servi de base à la rédaction de l'article Cercopithéque du Dictionnaire universel d'histoire naturelle. 
deux sections et en quelques petits groupes secondaires dont l'admission me semble très-propre à faciliter la distinction et la détermination des espèces.

Dans le Synopsis que je vais donner des Cercopithèques, j'insisterai surtout sur ceux qui, ainsi que le Cercopithèque Delalande, appartiennent à la seconde section. Ce sont les seuls, en effet, avec lesquels on puisse être exposé à confondre ce dernier.

\section{PREMLRE SECTIOX}

C'ercopithèques à museau plus court et à formes plus svelles.

Ces espèces ont le naturel plus calme et plus doux que celles de la seconde section, et se rapprochent davantage des Miopithèques et des Semnopithèques.

\section{A. - Espèces à nez velu et blanc.}

Esw. 1. Le CERCOPITHÈQUE HOCHEUR, Cercopithecus nictitans, Erxter.

Synonymie. Guevon a nez blanc proéminent, Buff., Suppl., VIII, Sima nictitans, puis C. nictitans de tous les auteurs méthodiques, Illiger excepté, qui, croyant à tort cette espèce dénuée de callosités ischiatiques, en faisait un Lasiopya. - Hochevr, Audeb., Singes.

Caractéristique. Pelage d'un noir très-liquctć de jaune pâle olivâtre, sur les parties supérieures et latérales de la tête (y compris les pommettes) et du corps; d'un gris noirâtre un peu tiqueté inférieurement: le menton blanchâtre; les membres ceta queue noirs. 
Les poils du devant de la tête et des joues s'allongent et se relèvent un peu chez les individus complétement adultes.

IIabitat. La Guinée.

Esp. 2. Le C. BLANC-NEZ, C. petaurista, Erxueb.

Syn. Buanc-nez, Allamand, Additions à l'Hist. nat. de Buffon;

Buffon, Suppl., VII. - S. petaerista, puis C. Pexaurista des auteurs méthodiques. - Blanc-nez et Ascagne, S. petaunista et S. ascanius, Audeb.-C. Petaurista et C. ascanius, Latr., Singes.

Car. Pelage supérieurement d'un vert plus ou moins lavé de roux, et tiqueté de noir; parties inférieures d'un blanc qui est très-pur sous la gorge et sous le menton; membres, en dehors, et queue, en dessus, d'un gris foncé, tiqueté d'olivâtre; le dedans des membres cendré; le dessous de la queue blanc.

Les poils du dessus de la tète sont allongés et un peu redressés chez les adultes.

Hab. La Guinée.

B. - Espèces a longs poils sur les parties latérales et inférieures de la face.

Esp. 3. Le C. A LONGUE BARBE, C. pogonias, Bennett.

Syn. C. pogonras, Bennett, Procecdings zool. Soc. of London, part. I, p. 67, ann. 1833 ; Lesson, Compl. de Buffon, deuxième édit., et Species; Ogilb., Monkeys.; Martin, Quadrum., fig.

Car. Une très-longue barbe d'un blanc jaunâtre, couvrant toutes les parties latérales et inféricures de la bouche, et tombant jusque sur le col. Pelage noirâtre, tiqueté de blanc, passant au noir sur le milieu du dos, la croupe, le dessus et l'extrémité de la queue; front et face externe des cuisses, jaunâtre tiqueté de noir; parties inférieures du corps et de la queue, et face externe des membres, jaune roussâtre.

Hab. Fernando-Po. 
C. - Espèces à queue d'un roux vif́.

Esp. 4. Le C. MOUSTAC, C. cephus, EnxL.

Sin. Moustac, Buff., XIV. - Moustache monkex, Penn. - Sim. ceprus, puis C. ceprus des auteurs méthodiques.

Malgré le nom spécifique qu’il porte dans tous les ouvrages, ce Singe n'est pas le Cephus des anciens.

Car. Tour de la bouche noir, avec du blanc immédiatement audessus du noir; dessus de la tête d'un vert lavé de roussâtre et tiqueté de noir ; des poils allongés formant une tache jaune assez étendue entre les yeux et les oreilles; dessus du corps et face externe des membres, d'un roux légèrement verdâtre, et tiqueté de noir ; mains, noirâtres ; corps et dessous du corps, blancs; face externe des membres, cendrée, de même que la région anale; queue, près de son origine, grise en dessous, d'un brun roux tiqueté, en dessus : le reste d'un roux vif, plus clair en dessous et vers l'extrémité.

La tache jaune des joues est d'un jaune doré chez les adultes, d'un jaune verdâtre chez les jeunes.

Hab. La Guinée.

Esr. 5. Le C. A OREILLES ROUGES, C. erythrolis, Wateri. Syn. S. erxtrinotis, Waterh., Proceed.zool. Soc. of London, VI, 58, ann. 1838 ; Mart.

Car. Pelage gris, les poils étant annelés de jaune et de noir ; joues et gorge, blanches; bras, noirâtres ; orcilles et région anale, rousses; queue d'un roux vif, avec sa ligne médiane supérieure et son extrémité, noirâtres.

Hab. Fernado-Po.

D. - Espèces ayant la queue de couleur variable, mais terne, et sans bande surcilière blanche ou blanchâtre.

Esp. 6. Le C. AUX LEVRES BLANCHES, C. labialus, Is. GEofF.

Syn. C. zabratus, Is. Geoff., art. Cercopithèque déjà cité, 1842 , et Mém. sur les Singes, dans les Arch. du Mus., 1. I1. 
Car. Pelage supérieurement long, bien foumi, d'un gris fonce, très-tiqueté de jaune pâle, olivâtre; une tache noire sur la face, au-dessus de la commissure des lievres; le reste du tour de la bouche, blane; gorge et parties inférieures, d'un blanc sale; les quatre mains et la face interne des membres de devant, noires; tour de l'anus et dessous de la queue dans une assez grande étendue, d'un fauve sale; dessus de la queue dans la même portion, varié de roux et de noir ; le reste de cet appendice, noir.

Mah. La côte occidentale d'Afrique. (?)

Esp. 7. C. CAMPBELL, C. Campbelli, Waterir.

Syn. C. Campelli, Waterh, loc.cil., part. VI, p. 61, ann. 1838; Mart.

Car. Pelage très-long; de longs poils sur les joues; poils se partageani et divergeant su le milieu du dos; parties supéricures, d'un gris olivâtre, les poils étant annelés de noir et de jaune; parties postérieures du corps et cuisses, d'un gris ardoisé, avec de très-petits anneaux; parties inférieures du corps et externes des membres, blancs; queue variée, en dessus, de noir et de jaune sale, en dessous, de noir et de blane brunâtre; les poils de l'extrémité, plus longs et noirs.

Hab. Sierra-Leone.

Esp. 8. Le C. MARTIN, C. Martini, Wateru.

Syn. C. Martin, Wat., ibid., p. 58 ; Mart.

Car. Poils assez longs; parties supérieures, grisâtres, les poils ètant annelís de noir et de blane jaunâtre ; dessus de la tête, bras et quene, noirâtres; poitrine, d'un blanc sale; abdomen et face exlerne des cuisses, brunaitres; des poils d'un brun rougeâtre a la base de la queue. (Espèce encore imparfaitement connuc.) ILab. Fernando-Po.

Esp. 9. Le C. 'TEMMINK, C. 'Temminckii, Ognus.

Syn. C. Тенmincki, Os., Mont., déc. 1838.

Cur. l'elage, superieurement, d'un cendré tiqueté de blane, avec: 
les membres noirs, le menton et la poitrine d'un blane pur, et le ventre cendré : la couleur de la queue n'est pas connue. Hab. La côte de Guinée.

Observation. Cette espèce doit être considérée comme très-douteuse : elle ne repose que sur un individu du Musée de Leyde, qui est en fort mauvais état, et n'a pu même être complétement derrit.

Esi. 10. Le C. MONOIDE, C. monoïdes, ls. Geoff.

$S_{1 n n}$. C. monoides, Is. Geoff. article Cercopithéque, 1842 , et $M \dot{c}^{-}$ moire sur les Singes, avec fig.

Car. Dessus de la tête et nuque, d'un vert olivâtre, tiqueté de noir; dessus du col, longs poils sur les joues, parlies inférieures des flancs, d'un gris tiqueté; dos et parties supérieures des flancs, d'un roux tiqueté, légèrement verdâtre; épaules, face externe des bras, avant-bras, les quatre mains, une partie des cuisses et la plus grande partie de la queue, d'un noir pur; devant de la poitrine et gorge, blancs; oreilles garnies supérieurement, à la face interne, de poils blancs, assez longs.

Hab......

E. - Especes ayant la queue de conleur variable, mais terne, et une bandc surcilière blanche ou blanchâtre.

Esr. 11. Le C. MONE, C. mona, Erxleb.

Syn. Mone et mova, Butf., Hist. nat., t. XIV, et Supplim., VII. -S. nona, puis C. mona des auteurs.

Mone, Mona, Monina, Mounina (d'où dérive vraisemblablement Monkey), est, d'après Buffon, le nom des Guenons dans plusieurs langues méridionales.

Car. Pelage olivâtre sur la tête, olivâtre clair sur les joues; une tache noire allongée de la partie supérieure de l'orbite à l'oreille, et une ligne d'un blanc verdâtre sur le devant du front ; dos, épaules, flancs, d'un roux tiqueté de noir ; croupe? noire, à l'exception de deux taches cllipliques, blanches, placées à droite et à gauche de l'origine de la queue; mains et 
face entière des membres, noires ; parties inférieures du corps et internes des membres, d'un blanc pur, qui, sur ceux-ci, tranche avec le noir de la région externe; queue variée de jaune et de noir, avec l'extrémité noire.

Il est à remarquer que les jeunes ont le dos roux olivâtre, et que le gris plus ou moins foncé remplace le noir sur les membres. Par ce dernier caractère, les jeunes se rapprochent de l'espèce précédente : mais on les reconnaîtra immédiatement par la lunule frontale, d'un blanc verdâtre, par les taches blanchâtres de la région fessière, et mieux encore par le système de coloration des membres, sur lesquels la couleur foncée des parties externes tranche nettement avec le blanc des parties internes.

Hab. La Guinée.

\section{Esp. 12. Le C. ROLOW AY, C. Roloway, Enxleb.}

Syn. Palatin ou Roloway, Allam., Additions à l'Hist. natur. de Buffon; Buff., Supplém., VII. - S. Rolowax, Schreb.; J.-B. Fisch.-C. Roloway, Erxl.; Zimmerm.; Less., Spec.

Car. Dos brun, trés-foncé, presque noir; tête, flanes, cuisses, jambes, d'un gris obscur, les poils étant terminés par une pointe blanchâtre; ligne sur le devant du front et barbe pointue, blanches; poitrine, ventre et face externe des cuisses, de couleur blanche, tirant sur l'orangé (quand l'animal est vivant).

Hab. La Guinée.

Observation. Cette espèce a été confondue par presque tous les auteurs avec l'espèce suivante, sous le nom de $C$. Diuna, qui appartient en propre à celle-ci. Ces auteurs se sont appuyés, pour réunir le $C$. Roloway et le $C$. Diana, sur l'insuffisance des caractères différentiels signalés par ceux qui ont admis comme distinctes les deux espèces. Les deux principaux de ces caractères sovit la disposition de la lusule blanche frontale, et la barbe bifide; et il est incontestable que ces caractères sont absolument 
sans valeur. Mais la couleur très-foncée et presque noire du dos (marron ou roux vif chez la Diane) et la couleur blanche du ventre (noir chez la Diane) distinguent bien le Roloway.

$$
\text { Esp. 13. Le C. DIANE, C. Diana, L. }
$$

Syn. Exoquma des habitants de quelques parties de la Guinée.-

Exequina, C. barbatus guineensis, Marcgr.-S. Diana, Lin., Mém. de l’Acad. de Stockholm pour 1754, avec fig. C. Diana, Erxleb.

Observation. Un grand nombre d'auteurs ont repris le nom de $S$. ou $C$. Diana, mais en l'appliquant en même temps à cette espèce et au C. Roloway. M. Fr. Cuvier l'a appliqué, au contraire, au $C$. Diana et au $C$. leucampyx, dont la description va suive.

Car. Parties latérales de la face, poils du menton, lesquels forment une barbe pointue et assez longue, gorge, poitrine, partie interne et antérieure de l'épaule et du bras, d'un blanc pur; une ligne blanche, étroite, à la partie antérieure du frout; milieu du dos, marron; ventre, noirâtre; flancs, d'un gris foncé, tiqueté de blanc; la queue noire, ainsi que les membres, sauf le dedans de la cuisse, qui est roux ou roussâtre, et une ligne longitudinale jaunâtre sur sa face entière.

Le Muséum d'histoire naturelle possède deux individus de cette espèce : l'un, nouvellement acquis, à cuisses intérieurement d'un roux vif (selon la description de Linné), et à ligne jaune sur la cuisse. Chez l'antre individu, conservé depuis fort longtemps, cette dernière ligne est faiblement indiquée, et le dedans de la cuisse est jaune. Cette dernière différence serait-elle un effet de la décoloration?

Hab. La Guinée, le Congo, Fernando-Po.

Esp. 14. Le C. A DIADÊEM, C. lcucampyx, MART.

Syn. Diane, vali, Fr. Cuv., Mamm., 1824. - Sim. leucamrux, J.-B. Fisch., 1829.-G. A uradême, C. diademalus, Is. Geoff., Zool. du Voyage de Bélanger, 1830-31;Less., Spec.-C. DIzophos, Ogilb., Monk., 1838.-Diadem Monkey, C. Ieucaupyx, Mart., 1841 . 
On voit par cette synonymic que M. J.-B. Fischer le premier, puis moi peu de temps après, nous avons déterminé comme une espèce distincte la prétendue variété de la Diane clécrite par M. Fr. Cuvier. Le nom spécifique lencampyx (mème signification que diadematus ) doit être préféré comme ayant l'antériorité d'une année.

Car. Dessus du corps et joues, d'un gris olivâtre tiqueté de noir; une tache en forme de croissant sur le front : cette tache est blanche, ainsi que le dessous du menton (mais non toute la gorge et la poitrine); queue non tiquctée de blanc; le reste, noir.

Hab. La côte occidentale d'Afrique (région encore indétruminée).

Obscrsation. Parmi les trois espèces que l'on avait confondues sous le nom de Diane, on voit que la première a toutes les parties inférieures blanches, tandis que cette couleur occupe seulement la gorge et la poitrine chez la seconde, et le menton chez la troisième : celle-ci, en outre, n'a point de barbe, et la lunule frontale est beaucoup plus grande.

\section{SECONDE SECTION.}

Cercopitheques à museau plus allongé et à formes moins sirelies.

Ces espèces ont le naturel plus pétulant et plus irascible que celles de la première section, et se rapprochent davantage des Cercocèbes (Mangabeys) et des Macaques.

\section{F. - Espèces a pelage gris verdâtre ou vert.}

On a désigné plusieurs de ces espèces sous le nom commun de? Singes verts.

\section{Esi. 15. Le C. DELALANDE, C. Lalandii, Noв.}

Syn. G. nane, de Lalande; C. pusizus, de Lalande, Desmoul., Dict.class. d'hist. nal., article Guenon, 1825.-C. Lalandi, Is. Geoft., article Cercopithèque, et Mém. sur les "Singes. Est-ce le $C$. slaucus, Lichtenst., loc. cit.? (simple mention, sans description, et mème sans phrase caractéristique). 
Car. Une bande blanche au-devant du front. Pelage long, d'un gris légèrement olivâtre sur le dos et les flanes; parties inférieures du corps et externes des membres, blanchâtres; la face, le menton et les quatre mains, noirs; queue grise, avec l'extrémité noire; l'anus cnlouré de poils ras, d'un roux vif. (Voyez. plus haut, p. 15, la description détaillée.)

Hab. L'Afrique australe, spécialement la forêt de l'Auteniquoi.

Oliserwation. Le $C$. Lalandii est distinct de toutes les espèces suivantes, en ce quil n'est véritablement pas vert, même sur le: dos et la tête, mais d'un gris à peine teinté de vertou d'olivâtre.

Esp. 16. Le C. VERVET, C. pyerythrus, Fr. Cuv.

Syn. Vervet, S. rygentura, Fr. Cuv., Mamm., 183 I; Desmoul., J.-B. Fisch.-C. Pygerytrir eus, Desmar., Suppl. i la Mamm., 1822.-Jard.-C. enxтropra, G. Cuv., Règ.an., deuxième édit. (nom que G. Cuvier attribae à tort à son fière).- C. r'gerxturos, Fr. Cuv., Mamm., deuxième édit.; Geoff. S.-H., Cours; Less.; Ogilb.; Mart. - Cercocebus pygeritureus, Smith, loc. cit.

Car. Une bande blanche au devant du front. Pelage d'un vert jaunâtre, tiqueté de noir sur la tête, le dos, les épaules, les flancs et le dessus de la queue; gris sur la face externe des membres. Parties inférieures du corps et de la queue et internes des membres, blanches; la face, le monton, les quatre mains dans leur totalité, le bout de la queue, noirs; tour de l'anus, d'un roux vif.

Hab. L'Afrique, région encore indéterminée. (V. plus haut, p. 13)

Observation. Très-distincte de la précédente par son pelago vort jaunâtre et non gris, cette espèce l'est de toutes les suivantes par la coloration du tour de l'anus, des mains, et surtout du menton; dernier caractère généralement omis d'aprẻs les descriptions antérieures. Le scrotum est vert-de-gris.

La description que je viens de donner, d'après l'individu type de l'espèce, est fort différente de celle des auteurs les plus récents. Parmi ceux-ci, les uns ont attribué au $C$. pygerythrus la plupart 
des caractères du C. Lalandii, qu'ils avaient seul observé, et qu'ils croyaient être le $C$. pygreryłhrus; les autres ont mélangé les traits des deux espèces, décrivant le C.pyggerythrus en partie d'après leurs propres observations, en partie d'après M. Fr. Cuvier.

Esp. 17. Le C. MALbrOUCK, C. cynosurus, Georf. S.-H.

$S_{j} n$. Sim. cyrosunus, Scopoli, Delic. flore et faun.; Schreb.; J.-B. Fisch.-Malmouck, Buff,, XIV (?); Fr. Cuv., Mammalogie.-C. MaLmouk, Latr.-C. crnosurus, Geoff. S.-H., Tableau des quadrumanes; Desmar.; Less., Complément et Spec.; Mart. - Cercocebus cynosurus, Jard.-Cercoc. Malbrouck, Less., Man. (et non Geoff. S.-H.") - C. TEPIrops, Benn., Procecd.zool. Soc. Lond., 1833, p. 109.-C. Faunus, Ogilb., Monkeys.

La plupart des auteurs rapportent aussi à cette espèce, mais sans motifs suffisants, le C. manbatus prous, Clusius; Sim. Faunus, Lin.; C. faunus, Erxleb.

Car. Une bande blanche au devant du front ( plus large, mais moins nettement caractérisée que dans les espèces précédentes et que dans la suivante). Pelage d'un vert très-jaunâtre, tiqueté de noir sur la tête, le dos, les épaules et les flanes; gris sur la face externe des membres. Parties inférieures du corps, $y$ compris le menton, et internes des membres, blanches; la face noirâtre, avec le tour des yeux livide; mains noires ou noirâtres, avec l'extrémité des doights plus claire; queue d'un gris noirâtre en dessus, blanchâtre en dessous; quelques poils roux autour de l'anus, ne formant pount une tache bien circonscrite. Mab. L'Afrique occidentale.

Observation. Cette espèce a, comme on le voit par ce qui précède, le bandeau blane plus large, le pelage plus jaune que le C. pygerythrus, et elle a le menton blanc; la face est, en outre, entièrement colorée; enfin, dans les mâles, le scrotum est d'un bleu lapis, et non vert-de-gris.

Quant à la différence de coloration du pourtour de l'anus, elle n’existe pas telleque l'a dit M. Frédéric Cuvier. Selon lui, le Ver- 
vet, nommé pour cette raison même $C$. pyergerthrus, aurait seul le pourtour de l'anus et le dessous de la base de la queue, roux; non-seulement ce même caractère existe, et tout aussi manifeste, chez le C. Lalandii, mais j'ai constaté aussi, sur un grand nombre de Malbroucks l'existence de poils roux à droite et à gauche de l'anus, et souvent aussi sous l'origine de la queue. 'Tantôt ces poils forment une tache distincte, mais beaucoup moins étendue que dans les deux espèces précédentes; tantôt ils se perdent sous les poils blancs un peu plus longs des parties environnantes, et il faut quelque attention pour les découvrir. Un seul Malbrouck ne m'en a présenté aucune trace, et encore est-ce un individu en mauvais élat, et ayant perdu une partie des poils de la région anale.

Esr. 18. Le C. GRIVET, C. grisco-viridis, Desmar.

Syn. Tota des Abyssins, et Aberzax des habitants du Sennaar (d'après Ruppell). - Grivet, Fr. Cuv., Mamm., 1819. - C. gritseo-virinis, Desmar., Mamm., 1820 ; Jard.; Mart.-Cencocebus griseo-vinidis, Less., Man., Jard. - Smia surviridis, Desmoul., 1825; J.-B. Fisch.-C. grisevs, Fr. Cuv., Mammif., deuxième édit.; Less., Compl. et Spec.; Ogilb., Monk.

Observation. Il est très-douteux que l'on doive rapporter à cette espèce le Six. exgrtutia de Hermann, Obs. zool.

Car. Une bande blanche, étroite, au devant du front; sur les joues, de longss poils blancs dirigés en arrière. Pelage d'un vert jaunâtre tiqueté sur la tête, le dos, les épaules et les flancs, d'un gris pur, liqueté de blanc, sur la face externe des membres; parties inférieures du corps, y compris le menton, et de la queue, et parties internes des membres, blanches; la queue, en dessus, d'un gris qui devient de plus en plus foncé, et passe au noir vers l'extrémité; la face noire, avec mains noirâtres. Point de poils roux autour de l'anus.

Le mâle a le scrotum vert-de-gris.

Hab. L'Abyssinie, la Nubie, le Kordofan, le Sennaax, l'Egypte. Observation. D'après la description sommaire qui précède, le 
C. griseo-viritis, quoique très-semblable aux deux espèces précédentes par l'ensemble de sa coloration, se distingue, $1^{\circ}$ du $C . p y-$ gerythrus, en ce qu'il n'a ni le menton et les mains dans leur totalité, noirs, ni la tache rousse de l'anus; $2^{\prime \prime}$ du $C$. cynosurus, par les longs poils de ses joues, par l'absence complète de poils roux à l'anus, autour duquel sont, au contraire, des poils blancs assez longs; par la bande blanche frontale, plus étroite et plus nette; et par la couleur verte du scrotum. Ces caractères de coloration sont sans doute de peu d'importance, et peuvent paraître insuflisants; mais ils concordent avee des différences très-marquées dans la forme de la tête, plus ronde et à museau plus gros chez. le $C$. cynosurus, pyramidale et à museau plus fin chez le $C$. grisco-viridis. Ce dernier est aussi généralement plus svelte ${ }^{1}$.

L'existence de poils orangés autour du scrotum n'est point un caractère constant.

Cette espèce est l'une de celles qui ont élé connues des anciens. On la trouve figurée sur plusieurs monuments égyptiens.

Esr. 19. Le C. CAllitriche, C. sabcus, Desmar.

Syn. Vulgairement Singe vent ou Sivge de saint Jacours.-Calnitriche, Buff., XIV.-S. sabza, Lin.; Schreb.; J.-B. Fisch. -C. samea, Erxleb., Latr.-Cencoceeus sabeus, Geoff. S.-H., Tabl. des quadr.; Less., Man.; Jard. - C. sabeus, Desmar., et tous les auteurs modernes.

Car. Bande blanche, peu distincte, au devant du front; face entièrement noire. Pelage d'un vert doré, passant au gris sur la face externe des membres et une partie de la queue; celle-ci tcrminée par un flocon de poils jauncs. Parties inférieures, blanches.

Le pelage plus vert die cente espèce, et la coloration de si

1 On woit qu'il 'est nécessaire de recourir, pour la distinction des C. cynosums et grisco-viridis, et generalement de toutes les especes de ce groupe, a des earacteres presque toujours négligés par les anciens auteurs; aussi la synonymie offre-t-elle ici les plus grandes difficultés, et reste-t-elle fort obseme of bouteuse, quelque soin que l'on porte dans l'interprétation des auteurs. 
quene, rendent sa distinction très-facile: coeš d'ailleurs le plus commun de Cercopithèques.

Observation. Le mâle a le scrotum vert, entouré de poils tantôt blancs', tantôt jaunâtres ou orangés ( mais non constamment orangés).

Hah. Le Sénégal et les îles du Cap-Vert.

Esp. 20. Le C. 'TANTALE, C. Thanalus, Ogiln.

S.n. C. Taxtalos, Ogilb., Proced. Soc. zool. of London, amn. 1841, p. 33.

Car. Une bande surcilière blanche. Pelage d'un vert olivâtre en dessus, cendré sur la face externe des membres; queue brunc, terminée par des poils jaunes; face noirâtre, avec le tour des yeux livide (espèce imparfaitement connue).

Hab.....

Esr. 21. Le C. ROUX-VERT, C. rufo-virilis, Is. G LOFE.

Syn. C. rufo-vinidis, Is. Geoff, article Cercopithéque, at Mém. sur les Singes, avec fig.

Car. Une bande surciliere blanche, dont la couleur contraste ave: celle de la face, qui est entièrement noire. Pelage d'un vert olivâtre sur la tête, d'un roux vert sur le corps, et d'un roux pur, à peine tiqueté de noir, sur les flanes, depuis les membres antérieurs jusqu'aux postérieurs. Les épaules et les cuisse’s, d'un gris verdâtre; le reste de la face externe des membres, gris; les mains antérieures, d'un noir tiqueté; les postérieules, grisâtres. Les parties inférieures du corps et internes des membres, blanches; la queue d'un gris foncé en dessus, ' d'un gris blanchâtre en dessous .

Hab. L'Afrique occidentale (?).

$$
\text { G. - Especes à pelage roux. }
$$

Esp. 22. Le C. PA'TAS, C. ruber, Geoff. S.-H.

Syn. Vulgairement Singe Rouge, - Patas a bandead norn et P. A bavdeau blanc, Buff., XIY. - S. liubra, Lin., Gm.-S. putas

ZOOLOGIE。 
et S. nufa, Schrels. -- C. nuna, Erxleb. - C. ratas, Larr. - C. rubra, Geoff. S.-H.; et tous les auteurs modernes.

Car. Pelage roux en dessus, blanc en dessous, d'un fauve grisâtre sur la partie inféricure des membres. $N e z$ noir.

Hab. Le Sénégal.

Esp. 23. Le C. A DOS ROUGE, C.pyrrhonotus, Ens. et EnR.

Syn. Nisnas des Ethiopiens. - C. pYrrnonotus, Ehrenb.; V hand. Gesellsch. naturs. Freunde, t. I, p. 183, 1829 ; Hemprich et Ehrenb., Symb. phys., 1830.

Car. Pelage roux en dessus, blanc en dessous et sur la partie interne des membres, partie postérieure du dos et dessus de la queue, d'un roux plus foncé que le reste du pelage; nez blanc. Hab. La Nubie.

Obscrvation. Cette espèce se distingue aussi de la précédente par la couleur des épaules et de la face externe des bras, qui sont roux comme le corps (et non gris ou d'un fauve grisâtre). L'une et l'autre ont d'ailleurs, à l'état adulte, exactement la même tailte; lassertion contraire n'a été émise que parce qu'on avait compare: le C. pyrrhonotus parfaitement adulte à des Patas encore jeunes '.

1 Bien que j’aie cru devoir admettre dans ce Synopsis quelques espèces encore fort imparfaitement connues, et même une qui reste douteuse, le $C$. Temminckii, je laisse entièrement en dehors du cadre de ce travail une espèce dont l'existence est parfaitement authentique, mais dont les rapports d'aflinité et la place naturelle sont encore tout-à-fait indéterminés; je veux parler du Semmopithecus albugularis de M. Sykes (Proceed. Zool. Soc. of Lond. 1850-51), reporté depuis par cet auteur lui-même et par MIt. Ogilby et Martin dans de genre Cercopithecus, près des C. Cynosurus et C. griseo-viridis, et considere aussi par quelques zoologistes comme faisant le passage des Semnopithèques aux Cercopitheques. Nayant jamais eu oceasion d'observer cette espece, ne la connaissant que par les descriptions des auteurs et par quelyues renseignements dus ì M. Gervais, je ne puis présentement que rappeler les diverses opinions imises a l'egard du Semn. ou $C$. albogularis, sans me prononcer is leur égard. 


\section{SECONDE PARTIE'}

SUR tes Singes a Méricarys conposaxt LES gexhes CALLITHRICHE, SMMIRI ET NYCTIPITHEQUE.

De même que les Singes de l'Ancien-Monde, ceux de l'Amérique se divisent en deux tribus entre lesquelles les genres et les espèces se répartissent fort inégalement ${ }^{2}$. L'une, celle des Hapaliens, ne comprend qu'un seul genre, Hapale d'Illiger, dont les espèces si remarquables par l'élégance de leurs formes, par la petitesse de leur taille et par la beauté de leur pelage, sont fréquemment apportées vivantes en Europe, et dont la connaissance est dès à présent assez avaucée.

A l'autre tribu, au contraire, celle des Cébiens, se rapportent tous les Singes que mon père, dans son Tableau des Quadrumanes, a compris sous les noms d'Hélopithèques et de Géopithèques; en d'autres termes, les Cebus et une partie des Ćallithrix d'Erxleben : groupes qui sont aujourd'hui, le premier sur-

${ }^{2}$ Cette seconde partie a été préscntée et en partie lue à l'Académie des sciences, dans sa séance du 29 mai 1843.

2 Les deux tribus des Singes de l'Ancien-Monde, selon la classification que j’ai exposée dans mon mémoire déjà cité sur lés Singes, sont celle des Pinnéciens, comprenant les trois genres 'Troglodyles, Pithecus et Hylobates; et celle des Cxnonminiciexs, daus laquelle se trouvent réunis tous les autres Singes africains ef asiatiques. 
tout, subdiviscis an un assez grand nombre de grenies.

Parmi ces genres, il en est quelques-uns que l'on peut considérer comme dojà conmus d'une manière assez satisfaisante. I'els sont, par exemple, les Atches de mon pere el mes biriodes, quoique ce dernier groupe ne soit chabli que depuis pen d'annces. L'únde de plusieurs antres genres, au contraire, loin quils aient été le sujet de travaux plus ou moins nombreux, est entièrement it reprendre. Pour les uns, il est vrai, tels que le genre Hurleur et surtout le genre Sapajou, nous sommes très-riches en matériaux; mais la difliculté du sujet est extrême; et, pour mit part, les ćtudes que j’ai faites sur ces Singes, m’ont conduit presque toujours, non it résoudre les dilicultés que j'avais d'abord apercues, mais tout au contraire it apercevoir, par une ctude plus attentive, des difficultés qui m’avaient d'abord ćchappé. A l'ćgard d'autres genres, tels que les Callitriches et les Nyetipithèques, les difficultés paraissent moindres; mass les matériaux sont rares el parfois manquent entièremeni.

C'est de ces derniers genres, c’est, en génciral, des Gépithèques de mon père que je vais moccuper dans ce travalt, ahu de mettre à profit pour la science

1 En laissant tontefois de côté le genre Saki, Pilhecia, qui n’a que des rapports assez doignés avec les autres genres.

J'ai traite ailleurs avec détail des Hélopithèques de mon père. Voyez Remarques sur les caracières généraux des binges américuins, el descriprion du nouveau genre Hiviodo, dans les Mím. du 
divers matériaux que les envois de divers voyageurs, mes propres recherches et parfois le hasard m'out procurés depuis quelques années, et qui ont triplé le nombre des individus dont j'avais pu disposer juscutalors.

\$I. Sur le genre Callithrix d'Erxleben et des auteurs qui l'ont surie

On sait que Bulfon divisait les Singes en cinq groupes; trois appartenant à l'Ancien-Monde, les Singes proprements dits, sans queue, les Babouins it queue courte, les Guenons à queue longue; deux autres appartenant au Nouveau-Monde, les Sapajous, i queue prenante, les Sagoins à quene non prenunte. Cette classification de Buffon, fort remarquable pour l'époque où elle fut proposée, est devenue le point de départ de tous les travaux ultérieurs sur la classification des Singes. Erxleben, en particulier, qui a été citć souvent comme l'un des fondateurs de cette partie de la méthode zoologique, n'a fait, dans son Systema regni animalis ${ }^{1}$, qu'adopter purement et simplement les cinq groupes de Buffon, en substituant aux noms de Buffon, fort ilifficiles à introdure

Mus., t. XVII, 1829 ; Description de teux especes nousclles de Singes à queue prenemte, ibid.; et article Sapajous ou Hélopithèques du Dict. class. d'hist. nat., t. XV, p.129-151. M. Le'sson m'a fait l'honneur de réimprimer en entier ce dernier travail dans son Complémene de Buffon, \&. IV, p. 159-225.

1 Lipsix, 1777. 
Jans la nomenclature latine, les noms suivants : Simia, Papio, Cercopithecus, Cebus et Callithrix.

Les caractères qu'assigne Erxleben à ses Callithrix, en d'autres termes, aux Sagoins de Buffon, sont au nombre de sept. Il ne sera pas inutile de les rappeler ici, et de présenter sur eux quelques courtes remarques.

Les deux premiers, tirés du nombre et de la disposition des incisives et des canines, et de même, le quatrième qui consiste dans l'existence de deux mamelles pectorales, bien loin d'appartenir en propre aux Callithrix, sont communs à tous les singes. Ce sont donc, non de véritables caractères génériques, mais, au contraire. des caractères de famille. Si Erxleben les a compris dans la caractéristique du genre, c'est parce que cet auteur, fidèle au plan qu'il s'était tracé, et dans lequel il n'a heureusement trouvé que peu d'imitateurs, divise immédiatement la classe en genres; d'où résulte pour lui la nécessité de comprendre dans la caractéristique de chacun de ceux-ci, avec les véritables traits génériques, les divers caractères ordinaux et sub-ordinaux.

Le troisième des caractères d'Erxleben, est l'exislence de mains aux quatre extrémités: manus in palmis plantisque. Ce caractère n'a pas plus que les précédents de valeur générique : il est, aussi bien qu'eux, commun à tous les Singes (du moins si l'on adopte la définition que j’ai donnce de la main ${ }^{1}$ ), ef

'Tappelle: main, toute extrénice poursue de doigls allongés. 
même aussi à tous les autres Primates. C’est donc un caractère très-général, un caractère d’ordre.

Les deux derniers caractères qu'Erxleben altribue i son genre C'allithrix sont l'absence des callosités ischiatiques ( nates tectre) et le défaut d'abajoues. $\mathrm{O}_{i}$, ces mêmes caractères se retrouvent chez les Cebus, 't ne peuvent encore fournir les éléments d'une disinction générique.

Il reste donc pour séparer les Callithrix, soit du

profondément divisés, irès-mobiles, trés-flexibles, et par consếquent susceptibles de saisir entre eux et la paume les objets placés à leur portée. Si ces objets sont légers et non fixes, l'animal peut les attirer vers lui, par exemple, les porter à sa bouche, ou les mouvoir dans toute autre direction; s'ils sont lourds ou fixes, il peut s'accrocher à eux, et s'en servir pour se mouvoir lui-même.

La définition que je viens de rappeler est très-différente de celles que l'on trouve dans tous les traités de zoologie. On y lit, en effet, que la main est constituée par la faculté d'opposer lc pouce aux autres doigls pour saisir les plus petires choses (définition de Cuvier) ; phrase dans laquelle je vois bien plutôt la description sommaire d'une main parfaite que la définition de la main en général. J'ai montré ailleurs (dans mon Mémoire sur les Singes) que, par l'adoption de la définition ordinaire, on se place dans la nécessité, ou de briser, en raison de quelques caraclères secondaires, l'unité de groupes viritablement naturels, ou de placer illogiquement parmi les caractères généraux des Primates des caractères que tous les Primates ne présentent pas. Entre ces deux inconvénients, également graves, Cuvier et presque tous les auteurs ont accepté de préférence le premier; et c'est ainsi qu'après avoir donné de la main une définition gui n'est applicable qu'à un ticrs environ des Singes, ils u'hésitent pas à les comprendre dans un ordre expressément caractérisé par l'existence de mains aux quatre extrémilés, et nommé, pour cette raison même, Quadrumanes. 
reste de la famille des Singes, soit plus spécialement, des autres Singes américains, un seul caractère : la queue prenante (prehensitis) chez les Cebus, n'est pas prenante chez les Callithrix. Et l'on voit que cet unique caractère est purement négatif, et, par conséquent ne saurait être, fût-il parfaitement exact, d'une grande valeur pour l'expression des rapports nacurels.

Aussi, ne doit-on pas s'étonner que le genre Callithrix renferme des espèces appartenant à deux types su'nciques assez differents pour qu'aujourd'hui nous soyons obligés de les répartir entre dewx tribus distinctes.

Sur les six espèces qu'Erxleben comprend sous le nom générique de Callithrix, une, C. pithecia, est le type du genre Saki, Pithecia, appartenant à la tribu des Cébiens; et tontes les autres font partie du genre Ouistiti, Hapale, qui compose à lui seul la tribu des Hapaliens.

En réunissant dans le mème genre des espèces que sépare, en réalité, un assez grand intervalle, Erxleben a d'ailleurs eu le mérite d'éviter une faute dans layuelle sont tombés la plupart des zoologistes venus après hui. Tous ses Callithrix ont bien la queue làche et non prenante : la caractéristique cauda elongata non prehensilis est exacte et applicable, sans exception, ì toutes les espèces qu'elle comprend, si différentes qu'elles soient d'ailleurs par leur systeme denlaire, la conformation de leurs doigh of de leurs ongles. 
Les classificateurs qui ont succédé à Erxleben, ont, au contraire, souvent réuni dans un mème groupe, soit sous le nom de Sagoins, soit sous celui de Géopithèques; ils ont caractérisé en commun par l'existence d'une queue longue, mais lache et non prenante, d'une part, des espèces chez lesquelles ce caractère existe en effet rigoureusement, telles que les Sakis et les Ouistitis, ou les Callithrix d'Erxleben; et d'une autre part, des espèces, telles que le Simia sciurea des anteurs ou Saïmiri de Buffon, et le Cobus Moloch de Hoffmansegg, chez lesquelles la quene est faible-ment prenante. Et ce qu'il importe de remarquer, c'est que ce sont ces dernières espèces placées, non sans raison, par Erxleben à la fin des Cebus, qui se trouvent comprises sous le nom de Callithrix par les auteurs du dix-neuvième siècle; par exemple, par mon père, dont la classification, publice sous le titre de Tableau des Quadrumanes ${ }^{2}$, a été très-généralement suivie; par M. de Humboldt dans son Tableau des Singes de l'Amérique" ; par G. Cuvier dans la première édition du Règne animal; par Desmarest dans la Mammalogie de l'Encyclopédie; par M. Lesson dans son Complément de Buffon, et par une foule d'autres.

Voici donc, par suite d'une véritable transposition

Loc. ril.

"Inséré dans le Recueil dobservations de zoologie, p. 357.-Le titre de ce volume porte la date de 181 ; mais le Tubleau des Singes placé à la fin du volume est évidemment postérieur d'une année au moins à 1811 , prisque l'illustre voyageur y cite le travail de mon père publićen 1812 dans les Annales du Muscum. 
du nom de Callithrix, deux sens fort différents attribués à ce mot. Les Callithrix d'Exxieben sont tous, pour les auteurs modernes, ou des Pithecia, ou des Hapale; et réciproquement, tous les Callithrix de cenx-ci, ceux du moins qui étaient connus d'Erxleben, étaient pour lui, non des Callithrix, mais des Cebus.

Pour achever de débrouiller celle synonymie gé nérique, il faut remarquer que, dès 1811 , une troisième acception était donnée au mot Callithrix. Pour Illiger, les Cébus de M. de Humbolrh, de mon père et des auteurs contemporains, étaient réunis au Saimiri sous le nom de Callithrix, singulièrement éloigné, comme on le voit, de sa signification première.

Enfin, la détermination que j'ai faite du Saïmiri de Buffon, comme type d'un genve distinct, vient modifier d'une autre manière encore, et cette fois par restriction, le sens du mot Callithrix, appartenant dès-lors en propre au Cebus Moloch de Iloffmannsegg (Callithrir Moloch, Geoff. S.-H. et Humb.), au Call. personatus de mon père, et aux autres espèces lices avec celles-ci par des rapports véritablement intimes.

En résumant tout ce qui précède, on voit que le genre Callithrix, tel qu'il doit être déterminé d'après les travaux les plus récents, correspond:

$1^{\circ}$ A une partie du genre Cebus d'Erxleben, et nullement à ses Callithrix;

$2^{\circ}$ Au genre Callithrix d'Illiger, moins le Simia capucina et les autres Sapajous, d'une part, et de l'autre, 
moins le $S$. sciurea, devenu le type du genre Sä̈miris ${ }^{1}$;

$3^{\circ}$ Au genre C'allithrix de mon père et des auteurs modernes, moins le $S$. sciurea.

Il est à remarquer que bien que le nom de Calliıhrix n'ait point élé appliqué d'abord aux espèces qui le portent aujourd'hui, il leur convient parfaitement, en raison de leur pelage long, touffu, abondant, et parfois remarquable par la beauté de ses couleurs.

$\$$ II.-Etat de la science en ce qui concerne les genres Saïmiri et Nyctipithèque.

M. Cuvier, dans la seconde édition du Rigne animal'2, sans ériger les Saïmiris en un genre nettement déterminé, en a fait, parmi les Sapajous, une petite section que l'auteur, non-seulement distingue bien des Callitriches, mais qu'il en sépare même par l'intercalation, d'ailleurs fort contraire aux rapports naturels, du genre Saki ou Pithecia.

Il ne sera pas inutile de citer ici textuellement le court passage dans lequel est indiquce cette première distinction :

"Dans les Saimris, dit Cuvier, la queue est dé-

'Illiger ne connaissait, et par conséquent il ne mentionne aucun des véritables Callilhix décrits par Hoffmansegg, par mon père, etc.; mais toutes ces espèces ne rentrent pas moins dans le genre Callihrix d'Illiger, d'après la définition quil en donne.

${ }^{2}$ T. I, P. 103 . 
" primée et cesse presque d'ère prenante; la tête est "très-plate"; il y a i la cloison interorbitaire du " squelette un espace membraneux. Nous n'en con" naissons qu'un, le Saïmiri (Gimia sciurea) Buff." "

Suit une courte description du pelage du Saimiri de Buffon.

Dans la traduction qu'il a domnée du Règne animal, M. Voigt ${ }^{2}$, en reproduisant le passage qui vient d'être cité, lui a fait subir une modification qui, tonte légère qu'elle est, mérite d'être mentionnce. Il a placé le nom de Saïmiri en titre, avant le passage qui vient l'être cité; et par-là, indiqué plus nettement la sépalation des Saimiris en un groupe distinct".

C'est en m'appuyant sur diverses observations de mon père ${ }^{4}$, que je considérai à mon tour les Saïmiris

${ }^{1}$ Ce caractère n'est pas exact. L'aplatissement de la tête forme, au contraire, l'un des caractères distinctifs des vrais Callitriches par rapport aux Saïmiris.

"Thiereirh. Leipzig, 1831.

"C'est ce qui explique comment MI. Lesson, dans son Species des Mammiferes bimanes et quadrumanes, p. 155, a cité M. Voigt, et non G. Cuvier, comme ayant le premier séparé les Sämiris des Callitriches.

"Voyez son Cours de l'histoire naturelle des Mammifères, 1829 , 1. 12 et suiv.-Voici ce que mon père dit du crâne du Saimiri; le passage est un peu long, mais il est bon de le citer ici en entier :

"Dire en termes généraux que le crâne du Saïniri contient, toutes proportions observées, le cerveau le plus volumineux, ne suflit pas à l'expression de ma pensée.... Dans le jeure sujet que voici, la boîte cérébrale forme un sphérö̀de de 18 lignes en longueur $\left(0^{m}, 0.10\right)$, de $16(0,036)$ dans la plus grande largeur, re de $15(0,034)$ en hanteus verticale. Je mesure ale plus tout le: crâne sur sa base, que je trouve de ag lignes (environ 0,060). Si 
Alans mes cours sur les Mammifères *, comme devant constituer, non une simple section, mais un genre, qui se distingue surtout ${ }^{2}$ par l'énorme volume du cerveau; caractère fqu'avaient omis, malgré sa haute importance, G. Cuvier et son traducteur M. Voigt.

J'ai fait voir en même temps que le genre Sä̈miris ${ }^{3}$, ne se compose pas seulement d'une espèce,

de cette quantité $27(0,060)$ vous soustrayez la longueur trouvere du sphérö̈de entier, ou le chiffre $18(0,040)$, vous avez $9(0,020)$, ou le tiers, pour la profondeur palatine de la face.

"Reprenons par parties la longueur de la base, afin de conuaître quelle est à son égard la position du trou occipital. Or, voici ce que doment nos mesures:

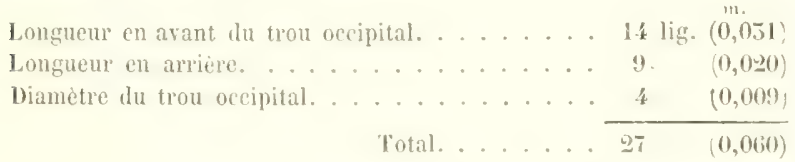

"Les mèmes mesures prises sur un crâne humain donnent ì peu près les mêmes proportions."

1 Voyez l'analyse que M. Gervais a publiée de mes leçons de 1835 sous ce titre: Résumé des Lecons de mammalogie professées au Muséum par M. Is. Geoffroy Sainl-Hilaire. Paris, in-8. Voyez. aussi l'Écho du monte savant.

2 Voici comment M. Gervais en indique, d'après moi, les caractëres (p. 19): Poils ras; oreilles plus petites et plus courtes que chez les Callitriches; et surtout développement très-considérable de l'encéphale, qui, proportion gardée, est peut-être plus volumineux que celui de l'homme. Les yeux sont extrêmement rapprochés, et la paroi interne des orbites est incomplete. Le tron occipital est situé au milieu de la base du crâne, disposition remarquable, et qui ne se trouve pas à un degré aussi élevé chez. tontes les races humaines.

JEt non Saimiri, comme on l'a imprimé par erreur. J'ai adopté Sä̈miris, à l'exemple des mots Indris, Loris, cte. 
comme on l'avait toujours dit, mais de trois. L'une de celles-ci venait d'être découverte par M. d'Orbigny, qui l'a figurée depuis. Une autre avait été rapportée plus anciennement par mon père, qui se l'étail procurée dans un voyage en Portugal.

Le groupe des Saïmiris a été admis, mais seulement comme une simple section assez peu distincte, dans l'Ostéographic' de M. de Blainville. Ce célèbre zoologiste décrit les Saïmiris comme de petites espèces de Sapajous, à queue extrêmement velue et à peine prenante, à tête longue et comme étirée, et offrant une particularité remarquable; l'état incomplet de la cloison interorbitaire, par absence de ce qu'on nomme l'os planum dans l'anatomie de l'homme, ou le peu de diveloppement des masses latérales de l'ethmoïde. L'auteur fait aussi remarquer que toute la tête est comme bulbeuse par la grande minceur des os et l'ćtat lisse de toutes ses parties. Quant au reste du squelette, il n'offre pas, dit II. de Blainville, de dilférences un peu importantes : c'est it tort que Daubenton avait cru at l'existence de trois vertèbres lombaires seulement; il en existe sept, le nombre des vertèbres dorsales était de treize.

En 4840, M. Lesson, dans son Species des Mammiferes bimanes et quadrumanes ${ }^{2}$, et de même, en 1842, dans son Tableau du Règne Animal, at

\footnotetext{
'Fasc. II, p. 17.1839.

Loc. rit.
} 
admis le groupe des Saimiris, toutefois en le considérant comme un simple sous-genre parmi les Sagoins (Saguinus de Lacépède) : sous-genre pour lequel l'auteur propose le nouveau nom de Pithesciureus, et dans lequel il n'admet qu'une seule espèce, le Saïmiri écureuil, Pithesciureus Sä̈miri.

M. Ponchet, dans sa Zoologie classique, en 1841, a aussi admis le genre Sämiri, mais sans lui donner de nom latin. Comme les auteurs précédents, il n'indique qu'une seule espèce.

On voit que, parmi les auteurs eux-mêmes qui ont cessé de confondre les Saimiris avec les Callitriches, il existe encore une très-grande discordance, soit relativement à la valeur de ses caractères, soit quant ati nom qu'il convient de lui appliquer.

La science est un peu plus avancée à l'égard dı genre Nyctipithèque, dont il me reste à dire ici quelques mots. On est au moins d'accord sur ses caractìres, et sur la nécessité de séparer les Nyclipithèques en un groupe distinct. La nomenclature seule varie selon les auteurs, et encore n'existe-t-il à son égard que de trèslégères difficultés.

Ce genre a pour type un Singe déconvert à Cassiquiare par M. de Humbold, décrit par lui sous le nom de Simia trivirgata, é érigé, dès 1811 , sous le nom d'Aòte, en un genre qui est aujourd'hui généralement admis. Par qui ce genre a-t-il été créé?

Par une circonstance singulière, les auteurs euxmêmes qui ont accompli ce progrès, loin de le revendiquer pour eux-mêmes, l'attribuent à d'autres. Le 
genre Aòre, selon II. de llumboldt ', appartient it mon père et à Illiger; et au contraire, selon mon père" et selon Illiger ${ }^{3}$, il appartient à M. de Humboldt.

Le fait est que ces trois auteurs ont contribué à l'établir. M. de thumboldt a seulement indiqué, dans son Mémoire sur les Singes de l'Orénoque ${ }^{4}$, une nouvelle famille de Singes, que l'on pourrait, dit-il, désigner' par le nom d'Aótes (ácoto:). (Quelques mois plus tard, Illiger a introduit dans le système le nouveau genre sous le nom d'Aolus. Enfin, en 1812, mon père en a rectifié les caractères sur un point important. M. de Humboldt, dans sa description, avait dit les oreilles externes presque nulles (auricula forè nullas), et de là le nom d'Aôte; Illiger, substituant à une indication inexacte une expression erronée, avait dit auriculce nullce; mais mon père, en cela suivi par M. de Humboldt dans son Tableau des Singes de l' lmérique ${ }^{5}$, s'est tenu dans les limites de la vérité en disant seulement les oreilles très-petites.

Le nom d'Aotus, d'abord adopté par plusieurs zoologistes ${ }^{6}$, est depuis plusieurs années entièrement abadonné. Deux noms nouveaux, successivement proposés, l'un, Nyctipithecus, en 1823 , l'autre Noc-

1 Loc. cil., p. 357 et 358.

- Tablcau des Quadrumancs, loc. cil.

P. 71.

4 Loc. cil., p. 306. 811.

"Publié à la fin du volume où se trouve le travail plus haut ilite.

"Desmarest, Mammalogie, 1820 ; Latreille, Familles naturelles du regne animal, 1825 ; et Jardiue, Monkeys, 1833. 
thora, en 1824 , se partagent les auteurs modernes.

Spix ${ }^{1}$ est l'auteur du premier de ces noms. Il a décrit sous les noms de Nyctipithecus felinus et $N$. vociferans, deux Singes dans lesquels, rompé par la caractéristique erronée d'llliger, il n'a point reconnu des Aotus. Aujourd'hui, il n'est pas douteux que le préterdu nouveau genre de Spix ne soit, sous un auure nom, le même que l'Alotus. H. de Humboldt nous fait connaître ${ }^{2}$ que mon père, dans un travail par lui communiqué à l'illustre voyageur, avait donué au Sakis ce même nom générique Nrctipithéque. Mais mon père n'ayant jamais publié ce nom, Spix a pu sans inconvénient le transporter aux Aotus. Hon père en a lui-même jugé ainsi, puisque dans son Cours sur Thistoire naturelle des Mammifères, il a admis la sub. stitution du nom de Nyctipithecus au nom d'Aotus.

C'est M. Frédéric Cuvier qui a introduit dans la science le nom de Nocthore, Nocthora ${ }^{3}$. Ayant eu occasion d'observer à la ménagerie du Muséum un Aotus vivant, et ayant constaté chez lui l'existence des conques auditives quil croyait n'aroir encore éti signalées par personne, ce célèbre zoologiste a cru devoir rejeter comme erroné, et comme propre à induire en erreur, le nom d'Aotus, et il a proposé le mot nouveau Nocthora.

En appliquant les règles de nomenclature que j’ai

1 Simiarum el Vespertilionum Species nor a, gr. in-fol. Munich, 1823.

- Loc. cil., p. 306 et 311 .

`Hist. nat. des Mammifères de la ménagerie, août 1824.

Zoorogie. 
cru devoir adopter et suive invariablement depris quelques années ${ }^{1}$, le choix à faire entre les noms proposés pour le Simia trivirgata et ses congénères, est exempt de toute difficulté. Le nom d'sotus, d'une part, comme exprimant un faux caractère, de l'antre, comme tombé en désuétude, doit être écar!é? Entre les deux autres noms, Nyctipithecus et Noc-

1 Voy., à la fin du mémoire déjà cité sur les Singes, les notes 11, III, IV et VII.

Parmi ces règles, dont la stricte observation pourra seule bannir de la nomenclature l'arbitraire qui y règne si déplorablement aujourd'hui, je citerai, en les exprimant de la manière la plus concise, celles qui sont relatives au choix des noms en général, à cause des applications que j'ai à en faire ici, et celles qui se présenteront par la suite presqu’à charque page.

1. Rejeler les noms absurdes par cux-mémes, ou contradictoires avec les faits ou les idées qu'ils sont destinés ì exprimer; car ils sont, proserits par la logique comne causes probables d'erreur.

11. Rejeter les noms déjà employés dans une aure arception: la logique les proscrit également comme causes probables de conlusion.

III. Considerer comme non avenus (toutefois en les citant en synonymie) les noms tombés en désuétude. En effét, ces noms n'ont véellement plus d'existence dans la science, et leur rétablissement entrânerait tons les mêmes inconvénients que la création de mots nouveratu.

IV. Suuf ces trois exceptions, entre plusieurs noms proposés, préférer inveriablement le plus ancionnement publié. La justice ot le respect envers les travanx antériems ne commandent pas seuls cette préférence : la logique la réclame aussi. On doir choisir le nom qui est le plus ancien, et non celui qui paraic le meilleur: car, sauf' des cas fort rares et exceptionnels, la dale d'un nom est un fair incontestable et incontesté; sa valeur peut être diversement interprite, selon les temps, les lienx at les doctrines.

2 Regles I et III. 
thora, le premier doit être préféré, cn raison de son antériorité ${ }^{1}$.

S III. - Des caracteres et des mours des Nyctipithèques.

Avant d'aborder l'étude comparative des caractères des Callitriches, des Satmiris ct des Nyctipithépues, i m'a parı utile d'ćcarter, à l'avance, quelques diflicultés terminologiques qui auraient pu se présenter, el notamment de fixer la nomenclature générique dont je devrai me servir. Dans une science où la nomenclature est si complexe et si immense, il importe de ne pas ajouter les difficultés des mots aux difficultés des choses.

C'est aussi afin de mettre plus de précision dans l'exposition des caractères et plus de clarté dans tout ce travail, que je place ici, en première ligne, le genre Nyctipithecus, celui de tous dont la détermination laisse le moins à désirer.

Son principal caractère, d'où dérivent les habitudes essentiellement nocturnes des Nyctipithèques, et quo leur nom rappelle indirectement, c'est l'énorme développement des globes oculares. Les Nyctipitheques, comme l'ont très-justement remarqué MN. do Humboldt ef Fréderic Cuvier, sont les Loris oumienx encore, les Cheirogales du Nouveau Monde ".

1 Règle IV.

"C'est, du reste, tout-à-fait à tort (qu'un zoologiste anglais a 
Les yeux, qui de nuit, dit' M. de Humboldt, ressemblent à des yeux de hibou, ont, d'après les observations de M. Frédéric Cuvier, les pupilles rondes. Les orbites, séparées par une cloison extrêmement mince et transparente mème, surtout en arrière, mais complète, sont d'une étendue considérable ${ }^{1}$, ainsi qu'on en jugera par les mesures suivantes, prises chez un Nyctipithèque el, comme termes de comparaison, chez un Sapajou et chez l'Homme.

Diamètre antéro-postérieur

Nucipitrièele. Sinimtro. Sajou. Homar. de la tête osseuse. . .

Diamètre transversal au niveau des trous anditifs. . $0,031 \quad 0,034 \quad 0,051 \quad 0,150$

Distance entre les parois externes des deux orbites. . $\quad 0,040 \quad 0,031 \quad 0,043 \quad 0,094$

Diamètre inféro-supérieur de l'orbite (hauteur) . . $0,019 \quad 0,015 \quad 0,022 \quad 0,032$

Diamètre transversal (largeur). . . . . . $0,019 \quad 0,014 \quad 0,019 \quad 0,037$

Diamètre antéro-postérieur (profondeur). . . . $0,022 \quad 0,020 \quad 0,025 \quad 0,045$ Moyenne des trois diamètrés orbitaires. . . . . $\begin{array}{llll}0,020 & 0,016 & 0,022 & 0,038\end{array}$

Rapport de cette moyenne au diamètre antéro-postérieur de la tête. . . . $\quad:: 1: 3 \quad:: 1: 4 \quad:: 1: 4 \quad:: 1: 5$

La simple comparaison de ces chiffres fait voir que chez le Nyctipithèque, si inférieur par les dimensions

récemment proposé de placer les Nyctipithèques parmi l's Iémuqidés.

2 Voyez la planche. 
générales au Sajou, les orbites ont cependant, à trois millimètres près, la même profondeur et la même hauteur, et, exactement, la même largeur ${ }^{1}$. Aussi la plus gramle largent lu crius des Nyctipithèques estelle donnée par le diamètre transversal pris au niveau des orbites, tandis que chez le Sajou, le crâne est beancoup plus large en arrière que la face ne l'est en avant dans la région orbitaire. Les differences que l'on trouve en comparant le crîne du Nyctipithèque à celui de l'llomme et à celui du Sä̈miri, sont analognes, mais, ¿ l'égard de l'Homme, bien plus prononcées encore.

Afin de mettre ces différences dans tont leur jour, on peut substituer à la comparaison des divers diamètres des orbites chez l'Homme, le Sajou et le Nyctipithèque, celle des surfaces des ouvertures de ces mêmes orbites, ou plus exactement des sections des cavités orbitaires au niveau de leurs ouvertures. Je mettrai, pour chaque espèce, en regard de ces surfaces de la section orbitaire, celle du grand trou occipital.

$$
\text { Nicistr. Sunetrit. Sasou. Iromur. }
$$

Surface de la section orbitaire. $271^{\mathrm{mm} c} \cdot 151^{\text {mm.c. }} 276^{\text {mm.c. }} 1049^{\text {mim.c. }}$ Surface du grand trou occipital. $\begin{array}{llll}53 & 53 & 91 & 877\end{array}$

'Le crâne que j'ai pris pour type est celui d'un Nyctipithecus felinus.

J'ai sous les yeux le crâne d'un autre Nyctipithègue, $N$. Icmurinus, chez lequel les orbites sont plus étendues encore que chez le précédent, et d'une largeur absolue plus grande que chez le Sajou. J'ai fait figurer ce crâne fort remarquable (voy. l'atlas); mais comme il est incomplet en arrière, j'ai préféré donner les mesures d'après un crâne de $N$. felinus, qui est en parfait état do conservation. 
D'où l'on voit qu'en prenant pour unité la sufface du grand trou occipital, la surface de la section orbitaire serait exprimé par les nombres suivants, savoir:

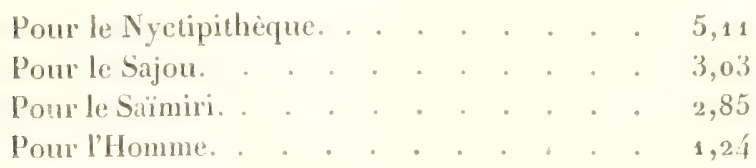

Les énormes orbites que je viens de décrire, occupent presque toute l'étendue de la face. Il n'existe qu'une très-petite distance entre le bord inférieur de l'orbite et l'arcade alvéolaire, et le museau est droit, court et un peu rentrant, de sorte que la ligue faciale qui est concave chez le Sajou, et que nous verrons être sensiblement droite cluez les Callitriches, et surtout chez les Saïmiris, est ici plus ou moins régulièrement convexe. La tête, portée sur un col court et gros, est donc dans son ensemble très-arrondie. Ce sont ces caractères qui ont f'ait dire à M. de Blainville ${ }^{1}$ que la téte en totalité rappelle un peu celle des Felis; et comme la physionomie extérieure résulte nécessarrement de la conformation interne, cette analogie avec les $F$ elis at frappé tous ceux qui ont vu des Nyctipithèques vivants ${ }^{2}$; par exemple, M. de Humboldt qui compare leur tête à celle du Chat-tigre, et Spix qui donne à

'Loc. cil., p. 20.

2 On dome même en quelques lieux, d'après M. de Humboldt, au $N$. trisirgatus te nom de Titi-ligre (Singe-tigre). Toutefois, M. de Humboldt dit que e’est en raison de la ressemblanee qui 'siste cutre le cri du $V$. Irivirgatus et celui du Jaguar. 
l'une de ces especes le nom de $N$ yctipithecus felinus.

Les narines, de forme ovalaire, sont en partie lalérales et en partie inférieures, la cloison internasale ayant peu de largeur. killes n'offrent done pas complètement la disposition qui a fait désiguer si souvent l'ensemble des Singes américains sous le nom de Platyrrhimus.

Bien que la tête soit, comme on l'a vu, beaucoup moins large en arrière qu'en avant, le cerveau est volumineux. Le crâne est, il est vrai, déprimé et trèspeu convexe supérieurement; mais la boite cérébrale est large et elle à de l'étendue en longueur, le tron occipital, qui est circulaire, occupant à peu près le milieu de l'espace compris entre l'occiput et les condyles de la mâchoire inférieure.

Celle-ci a ses branches montantes assez étroites supéreursment, mais très-dilatées infericurement; forme qui semble devoir être liće à un développement assez marqué de l'appareil hyoüdien et du larynx.

Les dents offrent la même conformation que chez les Sajous, remarque déjà faite par M. Fréd. Cuvier. Toutefois les canines ne paraissent jamais prendre le même accroissement que chez ceus-ei.

Les formes générales semblent lourdes chez l'animal vivant, en raison de son pelage très-long, trèsabondant, très-touffu. Mais elles sont en réalité trèslégères et très-sveltes, et, sous ce rapport encore, l'animal rappelle les Loris. Comme chez ceux-ci, la région lombaire, en particulier, est remarquablement allongrée; elle se compose de huit vertèbres, nombre plus 
considérable à na cumaissance, du moins, que chez ancun autre Singe.

Les membres antérieurs sont de hauteur moyenne, les postérieurs beancoup plus developpés, ainsi qu'or en jugera par les mesures suivantes.

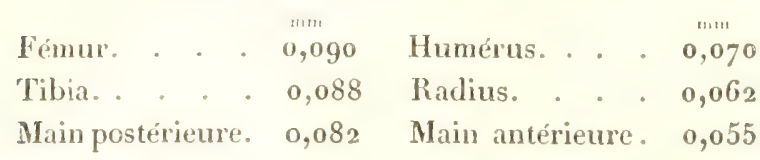

La queue, qui forme à peu près la moitié de la longueur totale, est très-velue et non prenante. Aussi les vertèbres caudales, très-allongées et très-grêles, res-semblent-elles beaucoup à celles des Ouistitis.

Les doigts que l'animal tient presque toujours demifléchis, surtout aux membres de devant, sont assez longs. Les pouces antérieurs sont à peine opposables, et leurs ongles sont seulement un peu plus larges que ceux des autres doigts : ceux-ci sont repliés sur euxmêmes, et conformés en gouttières; ceux des pouces antérieurs sans être précisément en gouttière, sont de même très-convexes; ceux des pouces postérieurs sont au contraire très-aplatis.

Enfin, pour achever de faire connaitre les caraclères génériques des Nyctipithèques, je dois mentionner leur pente taille, très-inférieure à celle des Sijous, et à peu près la même que celle des Saïmiris.

Ces remarques ne sont sans doute pas suffisantes pour faire nettement approuver les caractères des Nyctipithèques; mais ils seront bientôt comparés aux caractères des Saimiris et à ceux des Callitriches. On 
trouvera, en outre, les uns et les autres reproduits sous une forme concise et méthodique dans le Synopsis placé à la fin de ce travail.

Quant aux moeurs des Nyctipithèques, il me suffira, en renvoyant aux ouvrages de MM. de Humboldt, Spix et Frodéric Cuvier, de rappeler ici les faits principaux déjà signalés par ces auteurs.

Le plus important de tous, est relatif aux habitudes nocturnes de ces animaux. J'ai vu, ainsi que M. Frédéric Cuvier, le Nyctipithèque de la ménagerie, dormir presque constamment le jour, la tête replice sur la poitrine : habitude qui a valu au $N$. trivirgatus, sur les bords de l'Orénoque, le nom de Mono dormilon ou Singe dormeur. M. de Humboldt a vu un individu qu'il a possédé pendant plus de cinq mois, s'endormir assez régulièrement à neuf heures du matin, parfois dès l'aube du jour, et ne se réveiller qu'à sept heures du soir. La lumière, dit II. de Ilumboldı, l'incommodait beaucoup, et c'est aussi ce qu'affirme Spix, des Nyctipithèques du Brésil. Oculi ignei, dit-il, lucem perhorrescentes. C'est donc un fait parfaitement avéré que la vie nocturne des Ny ctipithèques, aussi actifs durant la nuil, aussi impétueux même, selon l'expression de M. de Humboldt, que le sont le jour presque tous les autres Singes, et aussi endormis le jour que ceuxci le sont la nuit. Il y a ici, comme on le voit, un accord parfait entre le trait le plus caratéristique de l'organisation du genre Nyctipithèque, l'énorme volume de ses yeux, et le fait le plus remarquable de ses habitudes.

Ce sont ordinairement des creux ou trous d'arbres 
quil choisit pou' gite. L'Tabitation su les artores résultant en quelque sorte comme conséquence nécessaire des conditions de l'organisation du Singe en général, et l'habitation dans les trous ou cavités obsenl'es se liant avec les habiudes nocturnes, on eût pu déduire a priori ce fait qui est d'alleurs attesté par M. de Numbolde. Au défint de trous les Nyctipitheques se logent parfois entre les grosses branches des arbres.

Ils sont it peu pres ommivores, ainsi que le sont la plupart des perites especes soit de Singes, soil de Lémuridés. Ils vivent en grande partie de fruits, saisissent avidement et adroitenent les insectes, et chassent parfois anx petits oiscaux. In captivite ils se monleent avides de friandises, et notamment de sucreries.

M. de Humboldt assure que les Nyctipithèues ne vivont pas en troupes comme les atutres Singes, mais deux ì denx dans une véritable monogamie. M. Spix nous représenteau contraire les $N$ y elipithéques comme vivant en troupes.

Lufin us demier fait avec lequel se lie manifestement la conformation remarguable de la mâchoire inforiene, signalée plus baut, c'est la force de la voix des Nyctipitheques: leur cri nocturne, dit M. de IIumboldt, d'une force vraiment extrardinaine, ressemble a celui du Jaguar, d'ou les noms de Mono-ligre et de Titi-tigre que l'on donne an $N$. Irivirgatus dans les missions de l'Orénoque ${ }^{1}$.

' L'animal, d'apres M. de Mtumbolde, a deux autres cris: 
Spix est beancoup moins explicite à cet égand que II. de Ilumboldt; mais le nom spécifique Vociferans, qu’il a donné à l'une de ces espèces, ne peut guère laisser de doute qu'il n'ait fait des observations analogues ì celles de son illustre devancier.

SIV. - Des Nyctipithèques décrits par les auteurs.

Avant de faire connaître les caractères de l'espèce nouvelle pour laquelle je propose le nom de $N$. lemurinus, j'analyserai le diverses descriptions dounées par les auteurs, et je chercherai ì en faire ressortir les traits et les caractèr es propres des sujets de ces descriptions.

La marche que je vais suivre, m'est prescrite par l'extrême difficulté du sujet; difficultó qui est telle que: je ne saurais encore espérer', dans l'état présent de la science, d'arriver à des résultats complétement satisfaisants.

\section{d. - Miriquouina d'Azara.}

Azara est le premier qui ait, sinon indiqué, au moins décrit, un Nyctipithèque. C'est incontestablement une espèce de ce genre qu'il décrit sous le nom de Miriquouina ${ }^{1}$, et non le Saki de Buffon ou une espèce

une espèce de miaulement, et un son guttural très-désagréable qu'il fait entendre lorsqu'il est irrité.

'T. II, p. 243 de la traduction française de Moreau de SaintMéry. 
voisine, ou cncore bien moins le Matrikina, comme l'ont cru Azara et tant d'autres après lui. Dans cette supposition, tant de différences se présentent entre les caractères réels et la description de Buffon, qu'Azara est obligé de relever successivement chaque trait de celleci, et qu'il accuse Buffon d'avoir décrit de mémoire et donné une planche arbitrairement faite.

Les traits caractéristiques, malheureusement un peu insuffisants, que l'on peut extraire de la description d'Azara, sont les suivants:

Cloison internasale peu large; caractère qui suffirait seul pour prouver que le Miriquouina appartient au genre Nyctipithèque, et non au groupe des Sakis, si remarquables par l'extrême écartement et la forme très-allongée de leurs narines '.

Longueur totale d'environ 87 centimètres, dont 38 pour la distance du bout du museau ì l'origine de la queue, et 49 pour la longueur de celle-ci.

Parties inféricures, y compris le dessous de la tête, face interne des membres antérieurs jusqu'aux coudes, et des postérieurs jusqu'aux genoux, d'une couleur cannelle qui, en dedans des fesses, est plus rougeâtre. Parties supérieures, d'une couleur mélangée que l'auteur ne détermine pas, mais qui est évidemment grisâtre, les poils ayant la pointe blanche, puis une portion noire, puis une autre portion blanchâtre.

Joues et menton, blanchâtres; au-dessus de chaque oeil, une tache de même couleur, peu prononcée, finissant supérieurement en pointe; milieu du front et face, de couleur foncée.

1 Un passage de la description pourait toutefois faire penser le contraire. "L'oreille, dit le traducteur, est très-large; dans son point le plus élevé, elle n'égale pas la hauteur de la tête. "Il faut entendre que le haut de l'oreille, qui est large mais courte, n'atteint pas le niveau cle la face supérieure du crâne. 
Poils de la queuc, noirs à la pointe, et le reste de conleur tabac d'Espagne faible, le noir occupant une portion d'autant plus grande du poil, et se prononcant d'autant plus qu'on approche davantage de l'extrémité de la queue. Les poils de la base de la queue ayant de 2 à 3 centimètres de long; ceux de son extrimité, de 4 à 5 .

Tel est le résumé de la description que donne Azara de cette espèce, découverte par lui dans la province du Choco, sur la rive occidentale du Patraguay.

Presque tous les auteurs, à l'exemple de mon père', en font un Saki sous le nom de Pithecia Miriquouina. M. de Humboldt, dans son Tableau des Singes de l'Amérique, lui a donné la même place dans le système, mais sous le nom de Simia Azarce. M. Lesson, au contraire, ayant reconnu en lui un véritable Nyctipithèque, l'a reporté dans le genre oì il doit rester.

B.-Douroucouli, Simia Irivirgata de M. de Humboldt.

Voici le résumé de la description domée par l'auteur de ce Singe, habitant, dit M. de Humboldt, les forêts épaisses du Cassiquare, près du village indien de l'Esmeralda, et les environs des cataractes de Mavpuras, entre le deuxième et le cinquième degré de latitude boréale.

Longueur totale d'environ 74 centimètres, dont 26 environ

1 Tableau des Quadrumanes. 
pour la distance du museau à l'anus, et 48 pour la longneru de la quene.

Gorge, poitrine, ventre et parties internes des extrémités, d'un jaune orange qui tire sur le brun; parties supérieures, d'un gris mêlé de blane, et comme argenté; les poils de cette partie, trèsdoux. Une ligne brume, longitudinale, sur le dos, depuis l'occiput jusqu’à la queue; une tache blanche au-dessus de chatque oil ; trois raies noirâtres, parallèles, sur la tête, et surtout sur le front. Visage couvert de poils noirâtres; nez pareillement noir, mais avec une ligne blanche, longitudinale, sur le milieu.

Quene touffue, de même couleur que le dos, à l'exception di: son cxtrémité, qui est noire.

Le Singe de nuit de Buffon est le seul Singe avec lequel M. de Humboldt ait comparé le Douroucouli, et il n'a point en de peine à prouver qu’il en diffère spécifiquement. Diffère-t-il aussi du Miriquouina? Il est impossible de rien affirmer ì cet égard, puisque nous n'avons, comme éléments d'une comparaison en elle-même fort difficile, que deux descriptions concises, et dont l'une est fort peu exacte. Cependant ha diversité spécifique de l'un et de l'autre me semble devoir être admise comme très-vraisemblable.

$1^{\circ}$ La coloration si caractéristique de la face el de la partie antérieure de la tête, chez le $S$. trivirgata, ne se retrouve pas chez le Miriquonina. On a quelquefois donté, il est vrai, que la figure de M. Mumbolds qui représente trois raies étroites ou lignes noires parallèles, suit parfaitement exacte. Mais le texte est aussi explicite que possible; les trois raies frontales, que l'illustre voyageu rappelle, comme le trait le plus caractéristique de l'espèce, par le nom de $S$. 
trivirgata, sont tellement remarquables, même pour les personnes étrangères à l'histoire naturelle, qu'elles ont foumi l'un des noms vulgaires. Les missionnaires de l'Orénoque appellent he Douroucouli Cara ray adu, c'est-à-dire face rayée.

$2^{\circ}$ Le $S$. trivirgata a encore quelques autres caractères distinctifs dans la raie blanche médio-nasale décrite par M. de Humboldt, dans la coloration de sa quene, et dans sa taille inférieure à célle du Miriquouina.

$3^{\circ}$ Enfin, il importe de remarquer que ces denx Singes viennent de régions que sépare une immense distance. C'est entre le second et le cinquième degré de latitude borrale que M. do. Ilumboldt a decouvert le Douroucouli; Azara avait trouvé le Miriquouina ver's le treizième degré de latitude australe.

C. - Singe de nuit à face de chat, Nyctipithccus felinus de Spix.

Voici la caractéristique que donne Spix pour ce Singe trouvé par lui au Para.

Sub-barbata, mystace ad latern mal.e disicho; corpore toto villoso-lanuginoso, suprà olivacco-cineraceo, sublies pallidè och raceo; raudú corpore muliò longiore, suprà radium cinerascente, subuis et versus latera usque ad medium rufescente, reliqua nigra; fuscia ni"râ urinqué $\dot{e}$ malis verticem versus clongala, alia nigra fronlis intermedia cum lateralibus confuente; maculis duabus suprì oculos albicantibus.

Les dimensions assignées à cette espèce par Spix sont, pour le corps et la tête, environ 16 pouces allemands, ou, en réduisant en mesures métriques, 0,376 ; pour la queue, 14 pouces, on 0,340 . 
Comme le fait observer Spix, ce Singe differe du Douroucouli par la non existence de la ligne blanche médio-nasale et par la moindre longueur de la queue. Ce dernier caractère distingue de mème le $N$. felinus du Miriguouina.

L'auteur fait seul remarquer que les poils du dessus du corps, d'un cendré foncé superficiellement, sont roux et noirs dans leur première portion.

La quene est presque toute noire, sa base étant seule d'un roux grisâtre en dessus, et ferrugineuse en dessous.

Enfin, parmi les caractères distinctifs de cette espèce, il importe d'insister sur la coloration de la tête. Il existe sur le milieu du front une tache noire, et sur chacun des côtés de la face et dı front une ligne noire dirigée de la joue vers le haut de la tête. Cette tache et ces deux ligaes correspondent évidemment aux trois raies qui ont fait nommer le Douroncouli $S$. Irivirgata; mais ici ce n'est pas une simple raie qui existe sur la ligne médiane, c'est une tache étendue. Spix se borne, il est vrai, à dire, dans sa caractéristique, qu'elle est confluente avec les raies latérales; et les deux descriptions qu'il donne, l'une en français, l'autre en latin, ne sont pas non plus suflisamment explicites. Mais la figure que Spix domne de son $N$. felinus, toute mauvaise qu'elle est, ne laisse aucun doute qu'il s'agisse ici, non d'une simple raie, mais d'unt: tache étendue.

D. - Babillard brun, Nyctipithecus voriferans de Spix.

Celui-ci, qui vient des forêts de 'Talatinga, au Brésil, près des frontières de ce vaste empire du côté du Pérou, peut, selon Spix, être ainsi caractérisé :

Imberbis, corpore tolo villoso-lanuginoso; capite rufescenti-brunneo; striis tenuibus i malis versus occiput ascendentibus, nigrobrunneis; maculâ ad frontis apicem nigricante, brevi, haud. confluente; alià utrinquè suprà oculum flavicante; caudà corpore vix 
longiore, ad radicem usque ad partem tertiam forrugineat; digitis palma planteque pedis longioribus.

Cette espèce, selon Spix, aurait à peu près les mémes proportions que le $N$. felinus : elle differerait donc notablement, sous ce point de vue, du Miriquonina, et aussi, quoigue M. Gray la considère comme identique avec celui-ci ${ }^{1}$, du S. trivirgatus. Ellc est représentée comme ayant les oreilles plus courtes que le $N$. felinus, dont elle diffère aussi par la couleur de la queue, d'un roux ferrugineux dans la première moitié, noir dans la seconde. Enfin, ce qui n'a lieu chez aucun des Nyctipithèques précédents, le corps est entièrement d'un brun qui, seulement, pâlit sous le ventre. Supérieurement les poils sont noirs à la racine, puis variés de roux et de noir.

Les joues, les lèvres et le menton sont blancs; une tache triangulaire, jaunâtre, est au-dessus de chaque oil ; une autre, semicirculaire et brunâtre, est en dehors de ce même organe. Le front présente, au milieu, une petite tache d'un noir brunâtre, et, de chaque côté, une bande étroite, presque filiforme.

\section{E. - Douroucouli de M. Frédéric Cuvier.}

Dans un Nyclipithèque qui a vécu à la ménagerie en 1824, M. Frédéric Cuvier a cru retrouver le Douroucouli de M. de Humboldt, que personne n'avait encore revu depuis cet illustre voyageur. Aucune difficulté ne s'élève en ce qui concerne la détermination générique; mais il n'en est pas de même de la détermination spécifique.

Le Douroucouli de M. Frédéric Cuvier est bien, comme le Douroucouli de M. de Humboldt, d'un cendré argenté supérieu-

1 Ann. of nat. history de Jardine, décembre 1842.

Zoologilt 
rement, "et d'un jatme assez vif inférieurement; mais il existe aussi plusieurs différences notab)les:

$1^{\circ}$ La queue ne fait guère chez le premier que la moitié de la longueur totale.

$2^{0} 11$ n'existe point de ligne foncée médio-dorsale.

$3^{\circ}$ Il existe bien trois tarhes noines sur le devant de la tête, mais les laterales peuvent seules être dites des raies, ta tache cenwale, qui est d'une assez grande étendue, citant aussi large que longue.

$4^{\circ}$ Le nez est entièrement noir, et les côtés de la face sont, ainsi que le menton et ane tache au-dessus de chaque oil, d'un roussâtre brun clair.

$5^{\circ}$. La queue, loin d'être de même couleur que le dos, est d'un roux ferrugineux, qui peu ì peu passe an noir, le tiers terminal environ étant de cette dernière couleur.

En présence de toutes ces différences, il y avait au moins lieu d'examiner si le Singe décrit par M. Frédéric Cuvier est bien le véritable Douroucouli de M. de Himmbolde.

L'origine de l'individu de M. I'r. Cuvier, venu vivant par la voie du commerce, est restée incommue; mais le même Nyctipilhéque a été envoyé de jhoxos au Muscum d'histoire naturelle par M. d'Orbigny, en sorte que sa patrie est aujourd'hui déterminée. Le Douroucouli de M. de Humboldt vient, comme on l'a vu, d'ume région fort diflérente.

En comparant de même le Douroucouli te M. Hr. Cuvier aux autres Nyctipithèques décrits, on wouve qu'il se rapproche beaucoup du Miriquouina par son mode de coloration, mais qu'il a des peoportions nolablement differentes. L'inverse a lien i l'egard du 
N. vociferans de spix, assez semblable par les pro portions, mais assez différent par la couleur.

Quant au $N$. felinus, au contraire, la comparaison très-minutiense que j’ai laite entre les descriptions ef la figure de Spix, d'unc part, et, de l'autre, l'individu lui-même qu'a décrit el figuré M. Fr. Cuvier, m’a pleinement convaincu de leur identité spécifique. Voici quelques-unes des bases de cette détermination:

$1^{0}$ La coloration caractéristique de la face est la méme. Il existe, en eflet, chez l'individu de M. Fréderic Cuvies', une tache noire médio-frontale, assez etendue, noire, ayant la forme d'un triangle dont la base serait en arrière. A droite, de chaque côté de cette tache noire, au-dessus de l'ocil, est une tache jaunâtre clain; plus en dehors, une ligne noirâtre, commencent sur la joue, re. montant vers te haut de la tète, et venant se terminer à l'angie postérieur de la tache midio-frontale.

Lindividu envoye de Moxos par M. d'Urìngy differe senlt:ment en ce que les taches super.oculaires sont d'un blanc sale, un peu jaunâtre, et non d'un jaunâtre clair, diffrrence qui est absolument insignifiante.

$2^{\circ}$ Le pelage, chez l'individu de la minagerie, est d'un cendré presque pur, un peu argenté sur les parties latérales du dos, d’un cendré olivâtre sur le milicu. L'individu de Mloxos offre de mème ces deux couleurs, mais la première seulement sur les épaules et la face externe des membres, et la seconde sur toutes les parzies supéricures, sauf le devant de la tête. Liudividu de Spix sessemblait plus sous ce point de vue à ce derujer qu'à l'individu de M. Frédéric Cuvier, peut-être pares que celui-ci était mort en cage après une assez longue captivité.

3"Cette esplication rend également compte, et ici je puis être beancoup plus aftirmatif, d'une différence existant entre la coloration de la quene chez l'individu de Spix et chez celui de M. Fr. Cuvier. Chez le premier, sauf quelques poils gris a la base, la 
queue est rousse dans sa première moitié, noire dans le reste; chez le second, le roux passe un peu moins promptement au noix: le dernier tiers environ est seul tout-à-fait de cette dernière couleur; mais il existe aussi, dans la partic qui précede, des poils noirs, seulement en plus pelit nombre. Le matuais fital du pelage de la queue explique cette légère différence; et je retrouve d'ailleurs, dans l'individu de M. d'Orbigny, tous les traits de la desw cription de Spix.

$4^{\circ}$ Quant à la couleur des parties inférieures, la description de Spix est également applicable de tout point aux deux individus que j'ai sous les yeux.

Ces analogies, et d'autres encore, relatives, soit à diver's détails de coloration, soit à l'étendue des oreilles, me mettent en droit de conclure que le Douroucouli de M. Frédéric Cuvier n’est point le véritable Douroucouli, $\boldsymbol{N}$. trivirgatus, mais le $\boldsymbol{N}$. felinus de Spix.

C'est par conséquent aussi à celte espèce quil faut rapporter les résultats des diverses observations faites par plusieurs zoologistes francais sur le squelette du prétendu Douroucouli de M. Fréd. Cuvier.

$$
\text { F. -- Résumé. }
$$

En admettant provisoirement comme espèces distinctes, ceux des Nyctipithèques, jusqu'à présent décrits, dont l'identité spu cifique ne peut pas être démontrée, ce genre comprendrait donc présentement :

$1^{\circ}$ Une espèce, de l'est du Brésil, que distinguerait surtout la non-coloration en fauve ou cannelle des parties inferieures, le $N$. rociferans de Spix.

$2^{\circ}$ Le $N$. trivirgatus, des bords de l'Orénoque, 
que caractcirisent, d'après l'ilitustre auteur quil l'a décrit, le pelage d'un cendré argenté supérieurement, la queue beaucoup plus longue que le corps, une ligne blanche médio-nasale, et surtout trois raies foncées sur la partie antérieure de la tête, enfin des oreilles très-courles ${ }^{1}$.

Cette espèce de Singe, aussi bien que la précédente, ne m'est connue que par une description el une figure.

$3^{\circ}$ Le $N$. felinus, du Para et de Moxos, à queue rousse et noire, seulement un peu plus longue que le corps, et à tache médio-frontale noire, assez étendue, à peu près aussi large que longue.

Cette espèce m'est connue, outre la description et la figure de Spix, par la peau et le squelette d'un individu, décril par M. Fréd. Cuvier et par plusieurs auteu's sous le nom de Nocthora, Simia ou Nyctipithecus trivirgatus, et par la peau d'un autre individu envoyé de Moxos par M. d'Orbigny.

$4^{\circ}$ Le Miriquouina, du sud du Paraguay, qui aurait le pelage du $N$. felinus, mais auquel Azara attribue une taille plus considérable et une queue plus longue.

Ce dernier, connu seulement par une description incomplète, et non encore figuré, est une de ces espèces vaguement indiquées, que l'on ne pent introduire,

1 C'est sans doute à tort qu'on a contesté ce caractère, dont l'expression toutefois semble avoir été un peu exagérée par M. de Humboldt. On verra plus bas que les oreilles sont, dans une partie des Nyctipithèques, notablement plus courtes que M. Fréd. Cuvier ne les a trouvées chez son Douroucouli, c'est-à-dire chen le $N$. felinus. 
même avec doute, dans le système, el quidoivent être mentionnées hors rang, en attendant de nouveaux éléments de déternination.

$$
\begin{aligned}
& \text { SV.-Descriplion d'une nouselle espèce de Iyctipi- } \\
& \text { thèue, N. lemurinus. }
\end{aligned}
$$

J'établis celte espèce sur l'examen des peaux'et des crânes de plusieur's individus des deux sexes et de différents âges, que le Nuscum d'histoire naturelle a récemment recus de Santa-fé de Bogota par diverses voies.

Le nom spécifique de Lemurinus que je dome i cette espèce, est destinć à rappeler, non-seulement les analogies générales qui existent entre les Pyctipithèques ut diver's genres de la famille des Lémuriens, mais aussi et surtout les caractères particuliers du pelage chez le $N$. lemurinus. C'est le mème poil, long, iouffu, lainenx, qui cxiste chez les Lemur, et c'est même aussi, quant aux parties supérieures la couleur la phus fréquente dans ce genre, notamment dans les plus communes de ses espèces. Ajoutons que c’est aussi ha même tendance it la production de variétés individuelles qui, sans apporter aucune modification importante à la distribution des couleurs, changent du moins d'une manière assez prononcée la nuance de andes-ci.

Dans le plus grand nombre des individus, les parties supérieures sont d'un cendré plus on moins lavé de youx et légèrement tiq̣ucté. Cette couleur cendrée se 
montre plus pure sur les parties latcirales du corps, plus rousse tout-i-inait en dessus.

Les poils de cette région, longs de 3 à 4 centimètres, ont tous leur bout noiritre, puis une portion assez ctendue cendré-roussatre, et la pointe annelée de fauve on de roussatre et de noir.

La couleur cendrée des flanes s'étend sans changer très-sensiblement de nuance, sur les côtés du col et sur la face externe des épaules, des bras et des cuisses.

Le dessus de la tête est d'un cendré plus ou moins lavé de roussâtre. En arant, au milieu du front, il existe une petite tache noire ou noirâtre, placée entre deux taches blanchàtres, plus étendues qu'elle. Le dessous des yeux et le menton sont blancs, le nez étant au contraire couvert de poils ras très-foncés.

ILe dessous du corps et la face interne des bras et des cuisses sont d'un jaune orangé ou roussâtre, plus vif sous le ventre, un peu moins sous la poitrine é en dedans des membres. Le dessous du col est seu. lement d'un cendré pâle roussâtre.

Les membres sont cendrés en dehors jusqu'aux mains; ils sont, en dedans, de la couleur de la poitrine ou du ventre, jusqu'aux coudes ou aux genoux, puis cendrés. Les mains sont couvertes de poils ras cendrés, fauves et noirs.

La queue près de sa base est, en dessous, rousse; en dessus, d'un cendré plus ou moins roussâtre qui se confond avec la couleur du dessus du corps. Le reste de la queue, couverte en dessus et en dessous de longs poils à base jaunâtre à extrémité noire, paraît tantôt 
d'un noir pur, tantôt d'un noir mêlé de jaune, selon qu'on apercoit ou non la portion jaune des poils sous la noire.

Sous la base de la queue, au milieu de la partie rousse, des poils noirs ou noirâtres, rendus secs, roides et comme agglutinés par la présence d'une matière grasse dont ils sont fortement imprégnés, et formant dans leur ensemble une tache ctroite et allongée, attestent la présence dans cette partie d'une glande assez développée. J'ai trouvé cette disposition chez tous les individus atultes soit maltes, soit femelles. L'analogue de cette glande existe chez le $N$. félinus, mais elle paraît moins développée, et les poils qui la couvrent, sont roux comme ceux qui les entourent.

I_es oreilles sont, dans cette espèce, beaucoup plus courtes que chez le $\boldsymbol{N}$. felinus. Je trouve la distance de la base libre de l'oreille, au point où l'oreille a le plus de longueur, égale à 9 millimètres seulement, tandis que je trouve 3 millimètres de plus chez le $N$. felinus, bien que ce dernier soit de plus petite taille ${ }^{1}$.

Nos individus adultes ont en effet tous de 36 à 38 centimètres dı bout du museau à l'origine de la queue, celle-ci ayant aussi à peu près cette même dimension. Je ne trouve au contraire que 3 i ì 32 centimètres chez les individus du $N$. felinus que j'ai sous les yeux.

J'ai dit au commencement de cette description que quelques individus m’ont présenté des variétés.

I Dans l'état frais, les oreilles seraient, sans nul doute, trouvées plus grandes; mais le rappor't subsisterait. 
L'un d'eux est remarquable par ses couleurs, généra' lement plus pâles et plus lavćes de roux. Il est plutôt fauve roussàtre supérieurement que cendré roussâtre, avec le dessous d'une couleur moins vive que chez les autres individus, et la queue, dans la plus grande partie de son étendue, d'un roux qui, même vers la fin, ne passe pas tout-à-fait au noir. La tache et le dessin de la partie antérieure de la tête sont les mêmes, mais la nuance diffère également : le dessus et le dessous des yeux, qui sont ordinairement blanchîtres, sont presque liures. Cet individu est femelle, mais une autre femelle m'a présenté les teintes ordinaires. II s'agit donc bien ici d'une variété individuelle, et non d'une différence sexuelle.

Chez d'autres individus, la variété résulte de l'étendue plus grande de la partie noire de l'extremitcides poils. J'ai observé cette modification sur le dos eı les mains chez un adulte, et sur le dos chez un très-jeune sujet, également remarquable et par l'extrême mollesse de sonpelage et par la conleur presque noire de son dos.

Ces variétés, quoique assez différentes pour la couleur, sont facilementriductibles ì leur espece, en raison des caractères très-constants que fournissent l'abondance et le mode de coloration des poils, la taille et les proportions, la distribution des couleurs, et notamment la disposition des taches de la face et du front.

C'est en ayant égard à ces caractères qu'on distinguera toujours le $N$. lemurinus et ses congénères, Savoir : 
$1^{\circ} \mathrm{Du} N$. vociferans, chez lequel le corps est entièrement d'un brun qui seulement pâlit sous le ventre;

$2^{\circ}$ Du $N$. trivirgatus, qui a la queue beaucoup plus longue que le corps, une raie médio-nasale blanche, et le pelage supérieurement d'un cendré argenté, avec une ligne foncée sur le milieu du dos;

$3^{\circ}$ Du Miriquouina, qui serait beaucoup plus grand, Qt aurail la quene proportionnellement beancoup plus longue;

$4^{\circ} \mathrm{Du} N$. felinus, qui a la tache médio-frontale beaucoup plus étendue, le pelage généralement beaucoup plus court, et notamment la queue beacoup moins ıouffue; chez lequel la couleur orangée ou roussâtre couvre inférieurement la gorge, aussi bien que la poitrine et le ventre; enfin, chez lequel aussi les oreilles sont plus grandes ou, plus exactement, noins courtes que chez le $\boldsymbol{N}$. felinus.

A l'égard de ces deux derniers Nycipithèques, je puis confirmer la différence rćellement spécifique de l'un el de l'autre par la comparaison de leurs crànes. Quelque grande que soit l'analogie de l'un el de l'au¿re, les différences suivantes peuvent être signalées :

Le $N$. lemurinus a les orbites sensiblement plus larges que hautes, el par suite proportionmellement un peu plus larges que chez le $N$. felinus, qui a le diamètre orbitaire transversal et le diamètre inféro-supérieur égaux entre eux: la différence est d'ailleurs peu marquée.

La boîte cérébrale est, chez le $N$. felinus, très-sensiblement moius large en arière qu'en avant; chez to 
N. lemurinus, elle conserve presque en arrière la même largeur qu'en avant. Ici encore la différence est d'alleurs lécire, at olfe elle-nime me confirmation de l'mbinite des rapports qui missent les derux cospees.

La mâchoire inféricure est la seule partie qui présente une diversité plus marquée. Chez le $N$. fétinus, la branche horizontale a les deux bords presque parallèles, la mâchoire étant de très-peu plus large audessous des demières molaires qu'au-dessous de la canine. Chez le $N$. lemurinus, la mâchoire dont, par suite, le bord inférieur est très-sinueux, est, au contraire, dilatée en arrière, beaucoup plus étroite en nvant. J'essaierai de rendre cette différence sensible par quelques mesures.

Distance entre les deux bords, prise au- $\times$ : exrnus. N. tmat nives. dessus de lir seconde molaire. - . . 99 millim. Distance au-dessous de la quatrième. . $\quad 9 \quad 10$ Distance au-dessous de la sixième. . . $10 \quad 14$

Cette inégalité, très-marquée, est un caractère tellement inhérent à notre nouvelle espèce, que je la trouve déjà indiquée chez un très-jeuneindividu, n'ayant encore que les vingt-quatre dents dela première dentition.

L'espèce que je viens de décrire, habite les Andes de la Nouvelle-Grenade, où elle paraît être fort commune, à en juger par le grand nombre d'individus qui nous sont tout à coup parvenus par diverses voies.

Parmi les voyageurs qui ont les premiers apporté en France lo $N$. lemurinus, l'uñ d'eux, M. Gondot, gui 
l'a plusieurs fois chassé, et qui l'a observé avec soin, a bien voulu, à ma demande, rédiger sur les moeurs de cette espèce une note, dans laquelle se trouve plusieurs détails intéressants. Je ne saurais mieux faire que de la citer ici textuellement.

"Ce petit quadrumane habite les grands bois de la région tempérée du Quindiì, dans la Nouvelle-Grenade, depins 1 foo mìtres, et mème bien plus haut. Il ne sort ordinairement ${ }^{1}$ qu'à la nuit tombante, vit en petits groupes ou familles, et ne parait pas s'ćloigner beaucoup de certains sites où il semble qu'il trouve facilement sa nourriture. Ces animaux font entendre presque continuellement, de nuit, lorsqu'ils vont dans les bois, un petit cri sourd, qui se trouve assez bien rendu par la parole douroucou, sourdement et faiblement prononcée, sans y ajouter l' $i$ (ainsi qu'il est écrit par M. de Humboldt.) Ils sont très-agiles. J'en ai vu qui venaient régulièrement chaque nuit dans les mêmes parages se nourrir des fruits de goyaviers, qu'ils paraissaient rechercher. Lorsqu'on leur tirait un coup de fusil, ils se retiraient, mais ne tardaient pas à revenir. De jour, ils restent cachés, et se trouvent réunis en petits groupes au sommet des arbres, non les plus élevés, mais les plus touffus. Peut-être même les amas de petites branches ef feuilles sèches qu'on trouve dans les lieux où ils se tiennent, y sont-ils réunis par

1 Je dis ordinairement, parce que, bien que je n'en aie jamais trouvé de jour, un chasseur m'a rapporté le fait qu’il en avait vu allant dlans l'après-midi.

Nore de M. Goudor.) 
eux. C'est dans ces sortes de nids qu'ils restent toute la journée à dormir. On a de la peine à découvrir ces gîtes, et lors même qu'on frappe contre l'arbre, ils ne se dérangent pas : ce n'est qu'en leur tirant des coups de fusil que je les faisais sortir de leur retraite: leurs mouvements ne paraissaient pas alors aussi vifs que pendant la nuit. La fernelle porte, comme celle de plusieurs autres quadrumanes, son petit sur le dos. Les habitants le designent sous le nom de Mico-dormilon.

"Bien que je n'aie pas vu ce même petit animal dans la Cordilière orientale, il parait hors de donte qu'il s'y trouve. M. le docteur Roulin en a vu un individu à Bogota, qui y avait été apporté des environs de la Mesa (village situé à une journée de la capitale), où ils sont aussi connus avec le nom de Micos-dormilones."

\section{$\$$ VI. - Des caractères et des mour's des Saïmiris.}

Les yeux sont grands encore chez les Saïmiris, comparés à leur développement moyen dans la famille des Singes; mais ils sont très-inférieurs en volume ì ce que nous venons de les trouver chez les Nyclipithèques. On a vu, par les mesures et les calculs domés plus haut ${ }^{2}$, qu'une section rle la fosse or hitaire faite au niveaudeson ouverture antérieure, s'est trouvée avoir pour surface, chez un Saimiri, environ 150 millimètres carrés, tandis qu'elle en a jusqu'à 27o chez un Nyclipithèque fort

1 Voyez p. 75 
peu different pas la taille. Lin prenant pour misc, cinez ces deux mêmes animax, la surface da grand trou occipital (qui était chez l'un et chez l'autre d'un pen peu plus de jo millincites carrés), on trouve, et la différence derient bien plus sonsible sous cette forme, que la surface de la section onbilaire représentée, clacz le Nyctipithépue, par le nombro 5, 11, l'est sculeneni, chez le Säimiri, par le nombre 2,85. 11 esi à peine nócessaire de farre remarguer que ce dennier nombre ne dépasse pas debeaucoup la moitić du premier.

Une antre différence, et plus remarquable encore, entre les Nyctipithégues et les Sämiris, est l'chat incomplet de la cloison osseuse inter-orbitaire, iresmince en aricine chez les premiers, mais partont ossim fiée : cette cloison est, an contraire, senlement menInaneuse chez les Sä̈miris, lans un espace de forme elliplique, el d'une assez grande étendre, qui correspond à la place ordinairement occupée par l'os planum. Il est donc me partie des orbites dans laquelle les deux globes oculaires viennent presque au contact, disposition fout analogue a celle que l'on observe chez une partie des monstres que j’ai désignés sous le nom de Cyclocéphaliens ${ }^{1}$.

Cet extrême rapprochement des ghlobes ocularies est ancore exprimé dans le squelette par une autre disposilion qui mérite d'être indiquée, la réunion ei, pour' ainsi dire, la fusion, ì leur partic postérieure, des reliefs que produit, à la base du crâne, la saillie des deux

- Historic ginerale des anonalies de lorganisation, t. II. 
orbites. Les trous optiques sont extromement rapprochés.

Il est fort remarquable que la perforation de la cloison inter-orbitaire s'observe chez les Saïmiris, dont les yeux sont d'un volume plus qu'ordinaire, mais non énorme, el qu'elle n'existe pas, comme on eût pu être porté à le supposer, chez les Nyctipilhèques, à globes oculaires si démesurément volumineux; mais, chez ceux-ci, les orbies se sont éterdues et, pour ainsi dire, dilatées en dẻors, comme le fait voir l'excès de leur saillie sur celle des parties postérieures du cràne ${ }^{2}$. Chez les Saimiris, au contraire, les orbites ne font pas en dehors plus de saillie qu'ì l'ordinaire, et la tête est généralement de forme droite et allongée; elle est. comme comprimée; modification d'où résulte nécessairement le rapprochement en dedans, la concentration vers le plan mécian de tous les organes latéraux.

L'allongement de la tête des Saïmiris résulte d'ailleurs, non-sevlement de sa forme comprimé, mais aussi, etsurtout, du développement considérable de la région occipilale; développement déjà signalé par mon père $^{2}$, et qui forme le trait le plus remarquable et

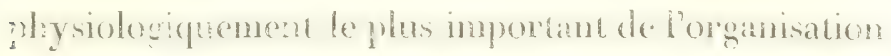
des Saiminis.

On sait que, chez presque tous les Singes, et les Nyctipithèques et les Callitriches sont eux-mêmes de ce nombre, la base du cràne se relève immédiate-

1 Voyez les mesures données plus lıaut, p. 52 .

2 Voyez plus haut, p. híf et 45. 
ment ou presque immédiatement en arrière du trou occipital, et remonte, soit obliquement, soit même presque verticalement, de manière à représenter bien plutôt une face postérieure que la contisuation de la face inférieure du cràne. Chez les Saimiris, au contraire, l'occipital presque tout entier est horizontal, et par conséquent compris dans la face inférieure du crâne; d'où l'existence derrière le trou occipital d'une ćtendue horizontale encore considérable. Cette étendue est telle que, chez l'adulte, la distance comprise entre les incisives et la partie antérieure du grand rou occipital, n'est que sensiblement double de celle qui sépare la partie postérieure de ce même trou de l'extrémité postérieure de la face inférieure du crâne. Chez les jeunes sujets, le cerveau étant plus développé encore, et la face plus courte, le rapport entre ces deux distances est moindre encore : selon l'âge, il est égal à un et demi, à un et un quart et même à un.

Voici quelques mesures prises comparativement sur deux individus, l'un adulte, l'antre jeune encore, mais déjà presque arrivé à la taille de l'adulte ${ }^{1}$.

J'exprimerai les diverses dimensions que $j^{\prime}$ 'ar à indiquer, non par les mesures elles-mêmes telles que les donne le compas, mais l'une manière plus facilement comparable, en fouctionsdudiametre antero-posterieur

- Ces deux individus appartiennent à l'espèce commune, ou selon le nom que je lui ai clomé, Saimiris sciureus.

J'ai sous les yeux une tête beaucoup plus jeụe; mais elle est en manvais état, et n'eut pu fumnir' les éléments d'uue comparaison exacte. 
Au grand trou occipital. La longueur de ce diamètre peut être prise pour unité, d'autant plus qu'elle est égale, aussi bien chez l'un que chez l'autre individu, à 8 millimètres; en sorte qu'en mullipliant par 8 les nombres ci-dessous, on obtiendra immédiatement les longueurs absolues, exprimées en millimètres.

Diamètre antéro-postérieur du grand trou occipital.

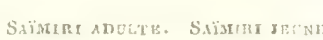

Longueur totale de la tête osseuse.. .

Largeur, au niveau du grand trou occipital. . . . . . . .

Distance de la partie postérieure du grand trou occipital à la partie postérieure de la voûte palatine. . .

Distance de la partie postérieure de la voûte palatine au bord des incisives.

Longueur de la portion de la face inférieure du crâne, en avant du grand trou occipital. . . . . . .

hongueur de la portion de cette mème face, en arrière du même trou. . . Distance de la partie postérieure de ce 11 $8,1 \quad 7,5$

$4,1 \quad 4,1$

$2,5 \quad 1,9$

$2,5 \quad 1,7$

$5 \quad 3,6$

$2,2 \quad 2$
mème trou à la partie la plus reculée de l'occiput ${ }^{1}$. . . . . . .

$2,5 \quad 3,1$

La portion de la face inférieure du crâne que je viens de désigner comme comprise entre le grand trou

I Chez l'adulte, à 18 millimètres environ du grand trou occipital, la paroi du crâne remonte presque tout à coup verticalement. Chez le jeune, à 16 millimètres, elle commence à remonter très-obliquement : d'ou la différence qui existe entre la terminaison de la fac inférieure du crâne en arrière et le point le plus reculé de l'occiput. Voyez les figures (PI. III)

Zootogre. 
occipilal el l'occiput, n'est pas moins remarquable par sa configuration que par. son ctendue. 11 existe chez l'homme, derrière le trou occipital, au milicu, un creux, latéralement, doux saillies assez prononcres. Chez les Saïmiris l'inverse a lien : il existe derrière l'occipital, sur la ligne médiame, une saillie longituảinale, el à droite ê à gauche sont deux fossettes très-marqućes; puis, plus en arrière, l'occipilal est très-renflé dans toute son bendue transversale. Ces indications, yne completera lialleurs el qu'éclaircira l'une des figures jointes it ce uém oire, sont suffisantes pour donner une idée de ce qui a lieu chrz le Saïmiri ordinaire ou sciurin. Miais, dans une autre espèce, désignée pal. moi sous le nom de Srïmiri à dos brûlé, ces divers caractères, que j’ai fait aussi représenter dans la planche, se presenteut avec un singulier degré d'exagéation : les fossettes que jai tout à l'heme mentionnées, sont tellement profondes, et la partie postévieure de loccipital est tellement saillante inférieurement, quil existe entre le fond des unes et la surface de celle-ci une difference the niveau égale à un demi-centimetre; nombre considérable cu égard anx proportions générales du crine, qui n’a guère plus de 3 centimètres et deni dans sa plus grande largeur.

Ce n'est pas seulement en arrière que le cràne des Sainiris présente un diveloppement remarquable: en avant, le cononal s'écive de beaucoup au-dessus du nivenu supérienr des orbites; en d'autres termes, if existe un veritable frons. Il laut remarifuer, toutefois, que ce front est fort different de celui de l'homme, 
qui est, comme chacm sait, concave au milieu, ef qui a latéralement ses maxma de saillie aux lieux disignés en anthropologie, pour cette raison même, sous le nom de bosses frontales. Chez les Sä̈miris, et il en est de même, au reste, comme je l'ai fait voir ailleurs', de tous les Singes qui ont un front, la plus grande saillie est située sur la ligne médiane, et correspond par conséquent, non au cerveau lui même, mais à l'intervallo compris entre les deux hémisphères, et par conséquent à l'insertion de la faux. A mesure que l'on s'écarte de la ligne méxliane, du moins chez l'adulte, le front devient ì la fois moins saillant en laut et en avant; il se déprime et se recule, et bientôt se coufond avec la saillie que forme l'extrémité supérieure du

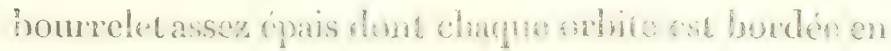
ঐlehors.

Encéphale.-J'avis depuis longtemp le lésir d'examiner l'encéphale contenu dims ce crâne si remarquable. Ce désir était devenu surtout très-vif chez moi, depuisque, pardiverses observations, dont la première remonte à 1840 , j’avais constaté l'absence des circonvolutions et des anfractuosités cérébrales chez les Ha-

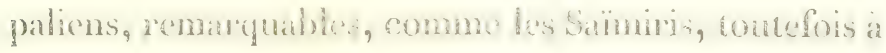
un bien moindre degré, par le volume de leur crâne. Devait-on penser que les Saïmiris auraient, comme les Hapaliens, le cerveau lisse? ê ces Singes, placés au premier rang entre tous, et à côtć de l'llomme luimême, si ce n'est au-dessus, par la masse proportion-

- Voyez mon Mémoire déjà cité sur les Singes. 
nelle de leur encéphale, devaient-ils descendre au dernier rang, et presque se placer au niveau des Rongeurs par la structure de ce même encéphale?

Deja M. Dismoulins avait repontu aflirmativement is cette question. Dans l'ouvrage que ce zootomiste a publié, en commun aver M. Magendie, sur les systèmes nerveux ${ }^{1}$, on lit qu'il n'existe pas de sillons au cerveau du Scämiri. L'auteur fait même de l'absence des sillons un caractère commun aux Singes américains, par opposition aux Singes de l'ancien monde, qui tous, dit M. Desmoulins, ont le cerveau plissé. Cette assertion est fort positive; mais il était clair qu'elle ne mérite aucune conflance. L'auteur mentionne, parmi les Singes amricains chez lesquels il aturait constaté l'état lisse du cerveau, l'une des espèces du genre Sapajou, le Saï, Celus capucinus. Or, à l'époque où écrivait M. Desmoulins, les circornvolutions et les anfractuosités cérébrales dı C. capucinus lui-mème, sans parler des observations faites sur d'autres espèces du même genre, se trouvaient déjà figurées depuis quatre ans par Tiedemann dans ses Icones cerebri simiarum ${ }^{2}$. Il y a plus: les Sapajous sont si communément amenés vivants dans nos pays, et les occasions de les examiner après leur mor't sont si fréquentes, que tous les zoologistes avaient pu examiner par eux-mêmes leur encéphale, et constater non-seulement l'existence, mais même le développement assez grand de ces cir-

A.T.I ( 1825$), 11.2,-19$.

2 In-fol. Heidelberg, 1822. 
Eurolutions nives par MI. Desmoulins. Ce zootomiste s'était done manifestement trompé à l'égard du Saï, et dès-lors il pouvait s'être trompé aussi à l'égard du Saimiri. J'ai dû d'abord ${ }^{1}$ les moyens de m'en assurer à l'obligeance do M. de. Blainville, qui voulut bien faire retirer, à ma demande, l'encéphale d'un Saïmiri sciurin, conservé dans l'alcohol au Musée d'anatomie comparée. Bientôt après, un autre individu de la même espèce, et tout récemment un autre encore étant morts à Paris chez des particuliers, j'ai pu me procurer deux autres encéphales de Saïmiris, et examiner ceux-ci d'une manière plus complète.

Sans donner dans ce travail, purement zoologique, des détails anatomiques que je réserve pour un mémoire spécial, je ferai du moins connaître la configuration générale de l'encéphale des Saïmiris. Son caractère le plus remarquable est sans contredit l'extrême développement de la partie postérieure des hémisphères. Le lobe moyen du cervelet est, il est vrai, très-développé et très-saillant en arrière; mais luimême est dépassé par les hémisphères céribraux de près d'un centimètre: les lobes latéraux du cervelet sont dépassés d'un centimètre et demi; ce qui est reladivement consirlirable, l'encéphale tout entier n'ayant qu'environ cinq centimètres et demi de long.

1 J'avais espéré d'abord trouver les tiléments qui m'étaient nécessaires dans une note anatomique sur les viscères d'un Saïmiri, inscrée dans les Proc of the zool. Soc. of Lond., part. I (1833), 1). 88. Mais l'auteur de ce travail, d'ailleurs intéressant, M. Marsin, ne dit rien de l'encéphale, qu'il paraît n'avoir pu examiner. 
En comparant le cerveau au crâne, on reconnaî́ que la saillie postérieure el inférieure que j’ai décrite plus haut, et qui est si prononcée surtout chez le Saï. miri à dos brûlé, correspond ì la saillie postériemre des hémisphères cérébraux, qui sont en ce lieu comme bombés. Quant aux fossettes, qui sont si profondes ¿ans l'espèce que je viens de rappeler, et que l'on retrouve moins prononcées, mais très-distinctes encore, chez le Saimiri sciurin, elles correspondent à l'intervalle compris de chaçze côté, entre la face latérale du lobe médian du corvete le lobe latéral de ce mène or gane et le lobe postérieur du cerveau.

Les hémisplières cérébraux finissent en avant plus en pointe que chez ies Sajous, genre dans lequel la coupe du corvear lop:ésente dans son ensemble une ellipse presque partate, ayant ses deux axes dans le rapport de 3 ì 2. Le rehaussement des hémisplères on avant, chez les wimiris, donne au cerveau la forme l'un ovale assez allongó p'utôt đụe đ'une ellipse.

Quant aux circonvolutions, is er existe quelquesanes chez les Sämiris, très-supérieurs par conséqueut, sous ce rapport, axix Oujstitis, mais très-sensiblement inférieurs aux Sapajous. Il en est surtout ains: des lobes antérieurs, dont la surface est lisse daus la plus grande partie de son étendue. Les lobes postérieurs n'ont, de même, que très-peu de circonvoluions; mais ce dernier caractère est commun aux Singes des trois dernieres a ribus.

Caractères extéricurs et dentaires. - Telles sont, dins leur ensemble, la conformation da crane el celle 
de l'encepplate chez les Sä̈mis; et nul antre genre, pas plus les Callitriches avec lesquels on les a si souvent confondus, que les Nyctipithèques ou tout autre groupe, ne partage avec eux ces caractères, notamment la perforation de la cloison osseuse inter-orlbitaire, et l'énorme saillie postérieure, soit de l'occipital, soit des hémisphères cérébraux. Si les Singes qui les présentent, ont été confondus avec d'autres Cébiens, c'est, sans nul doute, parce que ces caractères, quelque remarquables, quelque importants qu'ils soient, ne sont pas de nature à se traduire à l'extérieur par des modilications très-prononcées. L'extrême rapprochement, la presque contiguité des globes oculaires vers la partie moyenne el postérieure des orbites, n'est pas facilement appróciable à l'extérieur; elle est 1outefois indiquée 'par l'étroitesse de l'intervalle qui sépare les yeux en avant. Il en est à peu près de même des caractères résultant de la conformation générale du crâne et de l'encéphale : ils ne sont guère indiqués extériemrement que par la grosseur considérable de la tête, et notamment de sa partie postérieure, par la saillie du front, et par l'extrême brièveté de la face.

Les conditions organiques qui caractérisent fondamentalement les Saïmiris, élant de nature à n'être que très-imparfaitement'indiqués au dehors, il importe d'insister sur les caractères extérieurs qui concordent avec elles, et à l'aide desquels du moins it sera toujours facile de distinguer les Saïmiris.

Les narines fournissent, sinon à l'égard des Calliiriches, du moins à l'égard des Nyctipithèques, l'un 
de ces caractères ciminemment distinctifs. Hlles se pur sentent sous la forme d'ouvertures elliptiques placées tout-à-fait latéralement, et séparées par un intervalle assez grand entièrement recouvert de poils ras. Les Saimiris: sont donc du nombre des Singes auxquels le nom de platyrrhinins était justement appliqué.

Les oreilles, qui, en raison du développement de l'occiput, semblent ne pas occuper leur place ordinaire, sont médiocres. Elles sont de forme très-simple, la conque représentant un demi-cercle dont le bord supérieur se reploie sur lui-même.

Les dents, fort différentes, comme on le verra bientôt, de celles des Callitriches, ressemblent à celles des Sajous et des Nyctipithèques, mais avec quelques différences. Les principales de ces difléérences, à la màchoire supérieure, sont relatives à l'avant-dernière molaire, qui a la courome plus petite et plus simple, notamment moins étendue d'avant en arrière que chez les Nyctipithègues; et surtout anx incisives, placées, chez les Saïmiris comme chez les Singes supérieurs de l'ancien monde et chez l'Homme, presque exactement en ligne droite. Chez les Nyctipilhèques, la pare internédianm est placée beaucoup plus en avant. A la mâchoire inférieure, les incisives, et même aussi les canines, sont pareillement placées sur une ligne droite. L'avant-dernière molaire, aussi longue que large, est, comme à la mâchoire supérieure, plus pelite que la dent correspondante des Nyctipithèques; et, de plus, une diflérence analogue, mais beaucoup plus prononcée, existe entre la dernière molaire des Sitimiris, qui est fort fye- 
tite et presque rudimentaire, et la dernière molaire des Nyctipithèques. A l'une et à l'autre mâchoire, les canines sont, chez les vieux individus, saillantes, trèsépaisses à leur base, et creusées d'un sillon très-prononcé, placé, pour les supcrieures, à la face antérieure, pour les inférieures, à la face interne.

Entre ces différents caractères dusystème dentaire, le plus remaryuable est, sans nul doute, la disposition rectiligne des incisives. Au point de vue zologique, c'est un très-bon caractère à l'égard, soit des Nyclipithèques, soit des Callitriches; et sous un point de vue plus général, c'est un rapport fort curieux avec l'Homme et les premiers Singes, dont les Saïm ris, seuls entre tous les Cébiens, se trouvent reproduire en mème temps, à quelques rigards, less conditions criniennes et cérébrales, d'une part, de l'autre les caractères dentaires.

Ajoutons, pour terminer ici ce qui est relatif à l'appareil de la mastication, que la mâchoire inférieure des Saïmiris est remarquable par la forme de la branche montante, fort peu haute, mais très-large, et non dilaté posterieurement. Les branches horizontales ont un peu plus de hauteur en avant qu'en arrière. Chez les Nyctipithèques el les Callitriches, l'inverse a lieu, mais, chez les premiers, d'une manière peu marquée, et, chez les seconds, d'une manière si prononcée que la hauteur de la branche de la mâchoire est presque double au-dessous de la dernière moline de ce qu'elies est au-dessous de la première.

Les Saïmiris ressemblent beacoup plus aux Nycti- 
pithèques et aux. Callitriches par le corps et les memhres que par la tète, et il suftit d'indiquer succinctument. des caractères qui sont loin d'être aussi ramarquables que les précédents.

Les formes sont généralement grêles, ei les membres, strom les mans et les pieds, allongrs. Les onglesdes quatre doigts extomes sont en gouttière; ceux des pouces antérieur's sont très-petits et à demi-conformés en gontliere; certe des ponces pustricurs sont aplatis

La queue forme environ la moitié de la longueur totale. Elle est très-faiblement prenante et entièrement velue chez l'adulte en parfait pelage. Chez un très-jeune individu venant de la Nouvelle Grenade, je trouve au contraire la queue dénudée à son extrémité et sur toute sa face inférieure. Chez un autre plus avancé en àge, mais non encore adulte, je la trouve en partie dénudée.

Le phage qui rappelle celui de plusieurs singes de l'Ancien Monde, est généralement ras.

Enfin la taille est, comme chez les Nyctipithèques, ures-inferieure aux dimensions moyennes de la fimillo: des Cébiens.

Mocurs.-Je m'étendrai peu, en terminant ce paragraphe dejà très-étendu, sur les moeurs des Saïmiris. Le passage suivant de M. de Iltmboldt ', satt" un seul point sur lequel je reviendrai, résume à peu près tout ce que l'on sait de plus positif sur ces animaux.

"Leur physionomie est celle d'un enfant; même

1 Loc. cit., p. 333. 
"xpression d'innocence; même sourire malin, même rapidité dans le passage de la joie à la tristesse. Les Indiens assurent que cet animal pleure comme l'homme lorsqu'il éprouve du chagrin, et cette observation est très-exacte. Les grands yeux du Singe se motrillent de farmes à l'instant mème qu'ils marquent de la frayeur ou une vive inquiétude.

"Le Tili (Sä̈niri) est dans une agitation continuelle, mais ses mouvements sont pleins de légèreté et de grâce; il n'est jamais irrité comme le $S$. ocdipus ou le S. leonina. On le voit occupé à jouer, à sauter et à prenare des insectes, surtout des araignées qu'il préfèreà ious les aliments vegétaux. Il a l'habitude bizarre de regarcier fixement la bouche des personnes qui parlent; ê s'il parvient à s'asseoir sur leurs ćpaules, il touche de ses doights leme dents ou lem langue... I a sagacité de ce petit Singe est si grande, qu'un de ceux que nous conduisimes à San Tomas de la Nueva Guayana distinguail, parmi les différentes planches amnexées an Tableau élémentaire de l'histoire naturelle de Cuvier. celle qui présente les formes exterieures des insectes. Les gravures de cet ouvrage ne sont pas coloriées, et cependant le Titiavancait sa petite main dans l'espoir' de prentre une sautrelle, une gitepe, une demoiselle, chaque fois que nous lui présentions la XI ${ }^{e}$ planche ${ }^{1}$.

1 Des observations analogues ont été faites par M. Audouin à l'égard de l'Ouistiti ordinaire (voyez mon article Ouistili dans le Dict. class. d'hist. nat.), et plus récemment par moi-mème à l'égard de l'Ouistili à pinceau. 
Lorsque les Indiens tuent une femelle, atu moyen de leurs sarbacanes,... le petit Singe reste attaché à la mère, il tombe avec elle, et s'il n'est pas blessé par la chute, il ne quitte plus l'épaule ou le col de l'animal mort. La plupart des Titis que l'on trouve vivants dans les cabanes des indigènes, ont été ainsi arrachés au cadavre de leur's mères ${ }^{1}$."

Un point sur lequel je regrette de ne point trouver de renseignements dans l'intéressante relation de M. dellumbolde, c'est l'action de lat lumière sur les Sarmiris. M. Gama de Macharlo, qui a longtemps posscide et qui a observé avec soin un Sä̈miri, a rempli cette lacune, et constaté ${ }^{2}$ que l'animal fuit le jour et détourne les yeux d'une lumière trop vive. C'est, au reste, ce qu'on aurait pu déduire à priori de l'examen des yeux des Saïmiris, si inférieurs en développement à ceux des Singes nocturues, les Nyclipiliegues, mais cependant si remaryuables encore par leur volume.

1 La tendresse extrême des mères pour leurs petits et des petits pour leurs mères chez un Singe si remarquable par le développement des lobes du cerveau, est sans nul doute un fait très-conforme au cólèbre système de Gall; et les phrénologistes n'ont pas pas manqué de l'invoquer comme une preuve de plus en faveur de leur doctrine. On peut ajouter que cette tendresse réciproque des mères pour leurs petits et des petits pour leurs mères, trèsgénérale dans la fimille des Singes, paraît moins développée ou moins constante chez les espèces qui ont l'occiput déprimé, tels que les Hurleurs.

2 Thiórie des resscmblances, in-4, 1841 , p. 36. 
\$VII. - Des espèces du genre Saimiri.

Les auteurs n'ayant pas séparé les Saimiris des Callitriches, ont dû ne domner qu'une faible attention aux differences spécifiques qui peuvent venir modifier, chez. ces animaux, les caractères génériques communs ì tous. Aussi trouve-t-on décrits ou figurés, dans les auauteurs, sous le nom de Saimiri, Simia sciurea ou Callithrix sciureus, des animaux phus ou moins dille'rents. Dans cette espèce prétendue unique, je troure dès àprésent des él'ments sulfisants pour distinguer et caractériser rigoureusement trois espèces, et pour en indiquer une quatrieme. Les trois premières ont pour types:

A. Le Saïmiri de la Guyane, le plus commun dans les collections, et auquel appartiennent essentiellement les noms de Simia sciurea et de Callithrix sciureus. Cette première espèce sera donc pour moi le Sammiris sciureus, nom que je rendrai en liancais par celui de Saïmiri sciurin.

B. Un Saïmiri, habitant le Brésil, que mon père s'est, le premier', procuré en Portugal, dans un voyage fait en 1808 , et qu'il a décrit comme une variété du précédent ${ }^{1}$. Je lui domnerai le nom de Saïmiri à dos brûlé, Saïmiri ustus.

C. Enfin, un Saïmiri que M. d'Orbigny a découvert il y a peu d'années et qu'il a figuré sous le nom de Calli-

ITableau des Quadrumanes, loc. cil. 
thrix cntomophagus. Ce Sämiri, que les antems les phis récentsont rapporte commesimple varicto an Cullithrix sciureus, est bien réellement une espèce distincte, et doil conserver lenom d'entomop hagrus, quoique cenom exprime un fait de mocurs commun à tous les Saïmiris ", et même, plus généralement, à tors les petits Singes.

Outre ces trois espèces pour lesquelles j’ai sous les yeux des éléments suffisants de détermination, une quatrième se trouve indiquée par M. de Humboldt. On ne saurait, en effet, reconnaîle dans le Titi de l'Oréroque de cen illnstre voyagen, d'aprés la ciescription qu'il en donne, aucune des trois espèces précédentes. Je déngarai provinirement cente quatrieme: espèce sous le nom de $S$. lumulatus.

\section{A. - Saïmiri sciurin, Süumiris sciureus.}

Les caractères de cotte espèce, a ussi répandue dans les collections que les autres y sont rares, sont les suivants:

Pelage d'un gris olivàtre, avec le milieu du dos d'un vert olivitre tiqueté et tirant sur le roux, les côtés de la tête blancs ou blanchàtres, les parties inférieures d'un blane lavé de jamấtre, le dernier tiers ou le dernier quart de la queue noir, et les avant-bias, le bas des jambes et les pieds d'un jame roux plus our moins vif.

I En effet, si ce nom est peu convenable, il n'est du moins ni absurde, ni contradictoire; et clés-lors il doit être admis. (Règres I, II et IV; vovez plus hant, p. 50.' 
Lace couverte de poils ras, blancs, saul le tour de la bouche qui est noiritre.

Taille de l'adulte : trois décimètres et demi à quatre dicimèes dr bout du museau à l'origine de la quane, qui a environ quatre décimètres.

J'ai sous les yeux un assez grand nombre d'individus et de crânes de celle espèce, venant, la plupart, de la Guiane francaise, un autre du Para, un autre de Santa-Fi-de-\$3ogota, les autres de parties non determinées de l'Amérique méridionale.

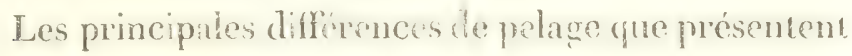
ces divers individus, sont les suivantes :

La queue est noire, tantôt dans le dernier quart seulement, tantôt dans le dernicr tiers de son étendue. Dans la partie qui n'est pas noire, le dessus est constamment d'une teinte plus foncée que le dessous; mais il arrive tantôt, et ceci a lieu surtout chez les adultes, que la différence soit peu sensible, le dessus étant d'un cendré olivître peu foncé, et le dessous d'un cendré fauve; tanlôt, au contraire, et c'est ordinairement le cas des jeunes sujets, que la différence soit très-marquée, le dessus étant d'un cendré olivâtre mélangé de noirâtre, et le dessous d'un fauve ou jaunàtre sale. J'ai cité plus haut un individu, le plus jeune de tous ceux que j'ai sous les yeux, chez lequel la queue est entièrement nue inférieurement : cette modification, qu'il faudra voir sur d'autres individus avant de se prononcer sur sa nature et sa cause, doit être rappelée ici.

La couleur des membres est, comme celle de la 
queue, susceptible de quelques variations. Ainsi quelques sujets, et particulièrement tous les jeunes, diffèrent par la moindre intensité, par la teinte plus verdâtre, et aussi par la distribution sur une moindre étendue du beau roux, souvent doré, qui orne les membres.

La face interne des cuisses et des jambes est tantôt d'un blanchàtre teinté de jaune, ce que l'on voit surtout dans le jeune âge, tantôt jame, mais toujours, surtout en ce qui concerne le dedans des cuisses, d'une nuance beaucoup moins vive que celle des pieds.

Quant au dos, il m’a présenté à peine quelques légères différences de coloration chez les indıvidus, d'àges et de lieux si différents, que j'ai examinés : sa couleur générale est toujours l'olivatre lavé de roux, et plus ou moins tiqueté de noir. Cette couleur résulte du mode de coloration des poils, qui ont, après la racine, colorée de gris noirâtre, une zone d'un jaune grisâtre, puis une zone d'un jaune roussâtre, à peu près de même nuance que les pieds, puis une zone noire, moins ctendue, immediatement avant l'extrême pointe, qui est jaunâtre.

\section{B. - Sämiri à dos brûlé, Saimiris ustus.}

Cette espèce a, comme la précédente, le dessus de la tête et les parties latérales du corps d'un gris olivâtre, les parties inférieures et le dedans des membres d'un blanc jaunâtre clair, la partie antírieure et externe des avant-bras et les quatre mains dorés, et la plus grande pardie de la face blanche, avec les leves noires. 
La queue offre aussi les mêmes couleurs semblablement disposées; seulement le noir occupe à l'extrémité une étendue un peu moindre.

La taille de cette espèce est un peu supérieure à celle du S. sciureus; mais les proportions paraissent être les mêmes.

Le Saïmiri à dos brûlé est donc, en somme, fort voisin du Sämiri sciurin; mais il s'en distingue nettement par la coloration des parties supérieures du corps. On a vu que le dos, chez le $S$. sciureus, est d'un vert olivâtre le long de la ligne médiane, mais non sur les parties latérales, qui sont grisâtres, comme les flancs, le dessus de la tête et la plus grande partie des membres et de la quene. Chez le $S$. ustus, le dos, aussi bien latéralement que sur la ligne médiane, est couvert de poils colorés par zones de trois couleurs, savoir, d'un jaunâtre sale à la racine, d'un roux ferrugineux sur la zone intermédiaire, assez étendue, enfin, noir à la pointe ${ }^{1}$, cette dernière couleur étant plus prononcée sur la partie médiane et inférieure du dos que latéralement. et antérieurement. De là résulte une coloration générale mélangée de roux et de noir, le roux dominant en avant et sur les. côtés, et le noir en arrière. Cette coloration, dans son ensemble, rappelle celle d'une étolfe inćgalement brûlée, seulement roussie dans quelques parties, et noire dans d'autres; et de là le choix que j’ai fait du nom spécifique d'ustus, déjà usité à

1 On a vu que chez le $S$. sciurcus les poils du dos ont une zone noire vers la pointe, mais que celle-ci est jaunâtre.

Zoorogif. 
l'égard d'un Bradype pour désigner un caractère fort analogue de coloration.

Les détails que je viens de donner sur la coloration du dos chez le $S$. ustus, sont suffisants pour en rendre la distinction nette et facile. Cette distinction est d'ailleurs confirmée par des différences ostéologiques beatcoup plus prononcées que celles que l'on pourait s'attendre à trouver entre des espèces extérieurement aussi voisines ${ }^{1}$.

I.e $S$. ustus exagère encor'e, en quelque sorte, les conditions cràniennes du genre Saïmiri, par la saillic vraiment singulière de la partie postérieure de l'occipital a la base du cràne. J'ai décrit plus haut² cette disposition, qui est telle, que la portion postérieure de l'occiput forme une sorte de poche ou de tambour osseux, dans lequel l'extrémité des hémisphères semble venir faire hernie hors de la cavité générale du cràne. J'ai mentionné aussi les fossettes très-profondes qui, à la face inférieure du crâne, séparent ce renflement sousoccipital de la portion de l'uccipital qui correspond au cervelet.

Le cràne du S. ustus diffère, en outre, de celui du S. sciureus par la longueur proportionnellement ${ }^{3}$ un peu plus grande du diamètre antéro-postérieur, par

1 L'individu, rapporté de Lisbonne par mon père en 4808 , sur lequel j’ai établi cette espèce, avait dans la peau un crîne parlaitement entier, que j’ai fait retirer afin de l'examiner, de le décrire et de le faire figurer.

2 Page 82.

3 La longueur absolue est de 72 millimétres au lieu de 65. 
l'étendue plus grande et la forme plus allongée du trou occipital, et par la plus grande longueur de l'arcade zygomatique.

C. - Sä̈iri entomophage, Saimiris cntomophagus.

Je me bornerai à indiquer succinctement cette espèce, que M. d'Orbigny a figurée dans l'une des premières livraisons de son voyage, et dont il doit donner prochainement l'histoire detaillée. Elle présente la mêne distribution de conleurstune le S. sciurent; mais, toutes ces couleurs étant fort affaiblies, c'est un jaune peu intense, qui remplace sur les membres antérjeurs et sur les mains postérieures le beau jaune roux doré du $S$. sciureus; et de même, sur le corps, un gris jaunâtre pâle, un peu plus vif seulement sur le dos, remplace les couleurs que j’ai décrites dans les espèces précédentes. La face a la même coloration; mais la nuque, tout le dessus de la tête, et, latéralement, de larges favoris, sont, chez l'adulte, noirs, et chez le très-jeune individu, noirâtres; les poils de cette région, d'un gris jaunâtre dans leur première partie, se terminent par une zone noire assez étendue.

\section{D. - Saïmiri à lunule, Saïmiris lunulatus.}

Le Titi" de l'Orénoque, de M. de Humboldt, a été

1 Dans l'Amérique méridionale, on donne ce nom, d'où dérive Ouistiti, non pas en propre à telle ou telle espèce, mais, en commum, à tous les petits Singes. Aussi l'applique-t-on, selon les pays, ¿ des espèces fort différentes.

11. de IIrumboldt fait à son oceasion des remarques très-judi- 
gencrabment rapponte, et cela a l'exemple de lillustre voyageur, au Simia sciurea de Limné, ou Saïmiri de la Guiane. H. Lesson, dans son Species, déjà cité, paraît être jusqu’à présent le seul qui ait fixé son attention sur les caractères particuliers que présente le Saïmiri de M. de Humbohdi:caracteres d'aprés lesquets M. Lesson a, en effel, séparé ce Singe, si ce n'est comme une espèce, au moins comme une variété distincte de tous les autres Sämiris connus.

Voici la caractéristique que donne M. de Humboldt. On y remarquera, comme le trait le plus remarquable de ce Saïniri, l'existence de deux lunules noirâtres sux la tête, lunules par lesquelles celte espèce se lie manifestement avec l'espèce précédente, sans pouvoir d'ailleurs être confondue avec elle.

"Simia sciurea (cassiquiarensis), ex aureo flavescens, abdomine, humeris, brachio et femore / nec antibrachio nec tibia) ex ferrugineo cinerascentibus... Frons cordata. Lunula duce nigrescentes ubi pili fusco-flavescentes frontem a sincipite secernunt.... Cauda corpore longior, haud prehensilis ${ }^{1}$, apice floccosa nigra.

cieuses sur les inconvénients qu'entraine l'emploi des noms triviaux comme noms spécifiques.

1 Il faut entendre par ces mots (l'analogie ne permet pas d'en douter) que la queue est très-faiblement prenante, ou, comme le dit M. de Humboldt qui plus bas aléveloppe sa pensée, sub-prehensilis. 
E. - Singe à tête de mort, Simia morta ou Capul mortuum des auteurs.

Si l'on devait en croire les anciens auteurs, à la suite du Simia sciurca devrait se placer une espèce fort voisine des Saïmiris par ses formes, ses proportions et les couleurs de son pelage, mais à queue nue et écailleuse : cauda nuda squamosa, disent Gmelin et les auteurs limnéens; cauda murina, dit Brisson.

Erxlében ${ }^{1}$ a pensé le premier que le Singe à tête de mort n'est pas une espèce distincte, mais seulement un foetus de Saimiri. Cette dernière opinion a prévalu parmi les auteurs modernes; et quelques-uns ont mème pensé que le Simic morla avait été ctabli sur un foetus de Saïmiri, auquel on aurait mis une queue de Didelphe.

L'examen que j'ai fait des divers éléments de la question, m’a convaincu que le Singe décrit sous lenom de morta est, non un foetus, mais un jeune âge de Sämiri, très-vraisemblablement dı Saïmiri sciurin. Lit quant a l'explication que l'on a donnce de sa quene nue et écailleuse, il n'est point nécessaire de recourir a la supposition d'une quene de Didelphe on de Rat, substituée artificiellement i la queue véritable. C'est ce dont chacun peut se convaincre facilement en remontant aux sources.

Les sources sont ici l'une des figures du Thesaurus

1 Systoma reanianimalis, [1. 53. 
de Séba, et le texte qui se rapporte à cette figure ${ }^{1}$. C'est, en effet, le Singe qu'il nomme Cercopithecus americanus minor Monkje dictus, et qui est connu aussi, remarque Séba, sous le nom de Caput morluum; c'est ce Singe qui a été inscrit dans le Systema naturce sous le nom de Simia morta.

Il est hors de doute que ce Singe n'est autre qu'un Saimiri : la figure est passable, assez bonne même, sauf la queue; et la description, quoique succincte, est fort claire, ainsi qu'on peut en juger par cette description des couleurs de la face: Tultus ommis pilosus est, et albicat, ad medium usque nasi et oris ambitum, ubi nigrido regnat.

Quant à la queue, elle est mal représentée, mais elle a, du moins, les proportions l'une queue de Saimiri, et je ne vois nullement dans la figure les écailles par lesquelles tous les auteurs ont cancterisé le Simia morta. Les traits croisés dans lesquels ils ont cru apercevoir des écailles, ont la plus grande similitude avec les hachures fort imparfaites employces habitudlement par plusieurs des graveurs de Síba. Au bas de la planche qui représente le Caput mortuum, on peut voir sur des feuilles et sur le terrain lui-même de tels traits croisés dans lesquels ici persomne ne mécommaitra de simples hachures.

Le texte ne mentionne d'ailleurs pas plus clairement les écailles que la lignrene les représente. L'auteur no dit rien de plus de la quene que ce qui suit : Cauda

1 T. 1, pl. XXXII, fig. 1; texte, p. 52. 
Tonga, crassiuscula, qualis Philandrorum aut Hurium majorum syloestrium : phrase qui, dans une description aussi précise, aussi vague, aussi incorrecte, peut tout aussi bien désigner une queue allongée, conique et nue, mais non écailleuse, qu'une queue absolument comparable à celle du Rat.

Or, si la queue était nue, mais non écailleuse, elle était telle qu'on pouvait s'attendre à la trouver chez un Saïmiri jeune âge, soit que les poils de la queue enssent été usés ou fussent tombrés, soit que la quene, chez de trés-jemes sujets, se trouve parfois naturellement dénudée dans son entier, et telle que je l'ai vue, dans plus de la moitié de son étendue, chez um Saïmiri sciurin, le plus jeune de tous les individus que j’aie observés.

\section{\$VIII. - Remarques sur les caractères du genre Callitriche.}

Le genre Callitriche, par la séparation des Saïmiris, devient un groupe fort naturel, dont le Moloch de Hoffmansegg et le Callitriche masqué de mon père peuvent-être considérés comme les types. Près de ces Singes viemnent se grouper plusieurs autres espèces, distinctes surtout par des difiérences dans la taille et dans la coloration.

Après les détails dans lesquels je viens d'entrer sur les caractères distinctifs des Nyctipithèques et des Saimiris, il ne suftirait presque de renvoyer, en ce qui concerne la caractéristiquedes vrais Callitriches, au $S$ rnopsis par lequel sera terminé ce travail. Cependant une 
comparaison suceincte de ces Singes et des Sainniris, et surtout de leur caractères crâniens, ne seront pas intutiles, afin de farre apprécier l'énorme différence existant entre les crânes de ces deux genres, si longtemps confondus entre eux.

Chez les Callitriches, le cräne proprement dit, comparativement à ce qui a lieu chez les Saïmiris, est considérablement restreint, et la face, au contraire, a pris beaucoup de développement.

Aussi, d'une part, en avant, le front est très-déprimé, ou plutôt, chez les adultes du moins, il n'existe pas de front. Immédiatement atı-dessus des orbites dont les bords sont ćpais et en bourrelets, il existe même, comme chez la plupart des Cynopithéciens, une dépression transversale.

La forme de l'occiput et la disposition du grand trou occipital, sont beancoup plus différentes encore de ce qui a lieu chez les Saïmiris. Au lieu d'être compris dans la face inférieure du crâne, fort prolongée même en arrière de lui, le trou occipital est plutôt postérieur yưuférien, la portion basilaire de l'os occipital étant seule horizontale, et tout le reste de cet os étant fortement relevé.

Dans la face, les orbites ont leurs ouvertures antérieures aussi larges que chez les Saïmiris; mais ces ouvertures sont séparées par un espace double de celui qui existe chez les Sammis. La cloison osseuse interorbitaire est complìte, comme chez tons les Singes, les Saïmiris exceptés.

La mîchoire inférieure présente un développement 
rès-grand, et, très-différente de celle des Sä̈niris, elle rappelle par sa forme celle des IIurleurs. Les branches montantes sout très-hantes, et inférieurement très-dilatées. Les branches horizontales, également très-hautes en arrière, se rétrécissent en avant, en sorte que les deux bords, loin d'ètre sensiblement parallèles, sont très-obliques l'un sur l'autre.

Cette forme de la màchoire est tellement remarquable et caractéristique à l'égard des Callitriches, qu'il importe de la rendre sensible par des mesures. Afin de rendre celles-ci comparatives, j’ai choisi, pour les placer en regard, deux crìnes dont la longueur absolue est sensiblement la mème. L'um appartient au Callithrix personatus de mon père, l'autre au Saïmiris ustus.

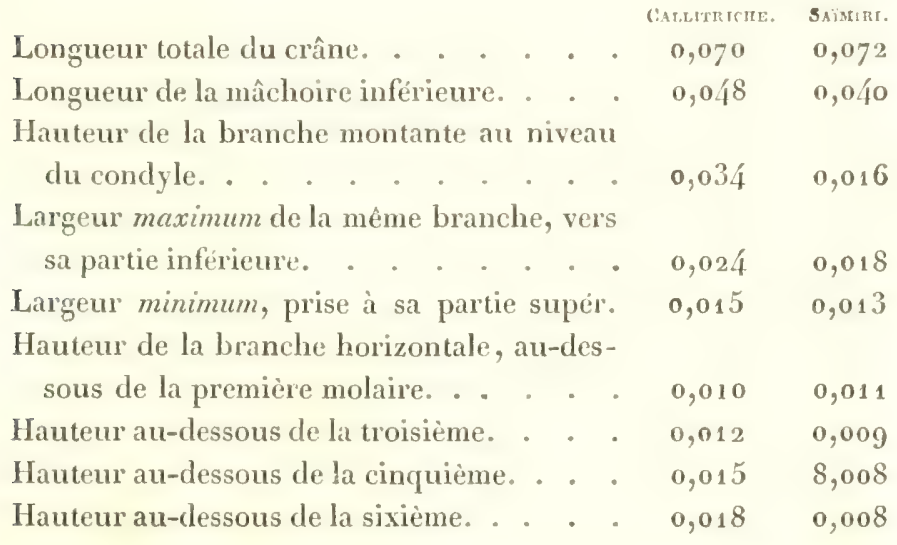

On doit s'attendre à trouver de très-grandes différences de dentition entre des genres chez lesquels la conformation de la mâchoire inférieure, ou mieux, des deux màchoires, est si différente, et même, i quelques 
égards, si manifestement inverse. C'est, en ellet, ce qui a lieu.

Chez les Saïmiris, les molaires supérieures, ce qui est parfaitement en rapport avec la brièveté des mâchoires, sont larges transversalement, mais, sauf la quatrième molaire, fort peu étendues d'avant en arrière; d'où il suit que leurs couronnes représentent des bandes beaucoup plus étendues daus un sens, le sens transversal, que dans l'autre. Les molaires supérieures des Callitriches, d'ailleurs à tubercules beaucoup moins pointus, ont leur courome plus rapprochée de la forme carrée, et surtout beaucoup plus grande; au point qu'en comparant de nouveau les deux individus dont je viens de parler, je trouve que la surface de toutes les molaires supérieures d'un coté, chez le Saïmiri, est tout au plus égale à la surface des trois dernières molaires chez le Callitriche.

11 existe à la màchoire inférieure des différences tout ì fait analogues; elles sont très-prononcces surtout à l'égard des deux derniè'es mâchelières, fort petites dans le genre Sä̈niri, grandes dans le genre Callitriche.

Aux deux mâchoires, les incisives des Callitriches sont disposées bien plus manifestement sur une ligne courbe que chez les Saïmiris. Immédiatement après elles, vient de chaque côté la canine, qui est courte et épaisse, et qui est contiguë en arrière, à la première molaire comme, en avant, à la seconde incisive. Toutes les dents, à l'une et à l'autre mâchoire, sont donc exactement en série continue, et leur ensemble représente une demi-ellipse parfaitement régulière. 
Le système dentaire offre donc, chez les Callitriches, comme la forme des michoires, comme la conformation du crine, et évidemment aussi comme celle de l'encéphale, des conditions qu'il est impossible d'assimiler plus longtemps it celles des Sämiris. Et même, entre ces deux genres, dont la différence était restée méconnue, il existe en réalité beaucoup plus de différences qu'on n'en observe d'ordinaire entre deux genres voisins.

On verra d'ailleurs par le Synopsis qui va suivre, et dans lequel on trouvera à la fois le résumé et le complément de tout ce travail, que la détermination extérieure des Sämiris et des Callitriches n'olfre point de difficultés réclles. Et si ces deux genres ont été si longtemps confondus, je ne crains pas d'afirmer que c'est à cause de la rareté de la plupart de leurs espèces dans les collections, et, par suite, du défaut de notions exactes sur leurs caractères.

SIX.-Synopsis des Sämiris, des Callitriches et des Nyctipithèques.

I. SAIMIRI, SALIIRIS 1.

Synonymic.

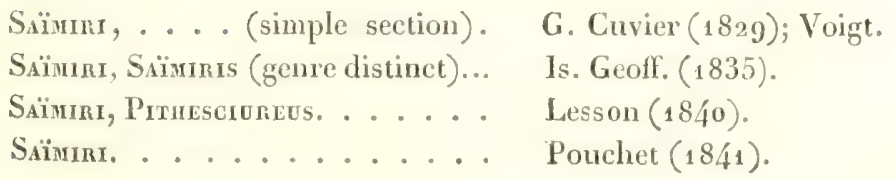

I It non Saimiri, comme on l'a imprime par erreur. 
Les Saïmiris, avant d'avoir été distingués génériquement, avaient fait successivement partie du grand genre Simia Lin., puis du genre Cebus Erxl. et en dernier lieu, du genre Callithrix, Geoff. S. Hil.

\section{Caractéristique.}

Formes légères. Membres allongés, les postérieurs beaucoup plus longs que les antérieurs. Pouces médiocrement allongés, les antérieurs à peine opposables. -Ongles des pouces postérieursaplatis, ceux des pouces antérieurs convexes; les autres en gouttière.-Queue longue, assez grêle, entierement velue (i l'ètat adulte), très-faiblement prenante.

Tête très-allongée, très-volumineuse, surtout en arrière. Occiput séparé des oreilles par une distance assez considérable, en raison du développement considérable du cràne et de l'encépluale en arrière du grand trou occipital. Front assez élevé au-dessus des or-bites sur la ligne médiane, mais, latéralement, fuyant et se confondant avec les bourrelets sus-orbitaires. - Face très-courte.

Yeux volumineux, très-rapprochés l'un de l'autre, et surtout en arrière où lacloison inter-orbitaire est seulement menbranense. - Conquesauricularesmoyennes, de forme simple. - Narines de forme allongće, latérales, séparces par un large intervalle. - Pelage peu fourni, principalement composé de poils amelés.

Incisives à la mâchoire supérieure, incisives et cani- 
nes inférienrement, dispostes en ligne droite. Canines longues, carénées. Canine supérieure de chaque côté, séparée de l'incisive externe par un intervalle qui recoit la canine inférieure correspondante, quand les màchoires sont fermées. Molaires à couronne médiocrement étendue, à tubercules les uns mousses, les autres aigus, surtout ceux de la partie externe. Molaires supérieures, disposées par rangées irrégulièrement rectilignes, sensiblement aussi distantes en avant qu'en arrière. Les supérieures, surtout les fausses molaires, transversalement allongées. La dernière molaire, de chaque côté et à chaque mâchoire, très-petite.

Taille très-inférieure à la taille moyenne des Singes.

1. S. sciurix, S. sciureus.

Sym. Vulgairement Singe écureule, Sapajou aurore OU ORANGÉ.

Saìna (nom formé du nom Galibi Kä̈-mirı), Buff. XV; Fr. Cuv. Mamm.; Gama de Mach. loc. cit. fig.Simia sciurea, Lin.; Schreb.-Cebus sciureus, Erxl.; J.-B. Fisch.-Callitriche Saïmiri, Callithrix sciureus, Geoff. S. H. Tableau des quad. et Cours; Desmar.; Less., Compl. de Buffon; Jardine, Monk., fig. - Sagoin Saïmiri, Saguinus sciureus, Less., Manuel. - Saïmri écureull, Pitiesciureus Saimiru, Less., Spec, et Nouveau Tableau.

C'est un jeune Saïmiri sciurin qui a été décrit sous les noms suivants: Cercopithecus Americayus, minor, 
Monkje dictus ou Caput montuur; Séba. - Sima morta des anciens anteurs.-Cebus capet mortudr, Fermin, Descr. de Surinam.

Car. Pelage d'un gris olivâtre, passant à l'olivâtre sur le dos. Les avant-bras et les quatre mains, d'un jaune roux doré.

Hab. La Guiane, la Nouvelle-Grenade et le Para.

2. S. A DOS BRUlé, S. ustus.

Syn. SAïmri, variété, Geoff. St.-Hil., Tabl. des Quadrumanes; Less., Species.

Car. Dessus de la tête et face externe des membres, d'un gris olivâtre; les parties supérieures du corps, d'un roux vif varié de noirâtre, passant au noir sur la partie postérieure et médiane du dos. Les avant-bras et les quatre mains, d'un jaune roux doré.

Hab. Le Brésil (d'après mon père, qui a rapporté de Portugal le seul individu qui me soit connu).

3. S. A Lunutes, S. lunulatus.

Syn. Bitschetscur des Indiens des Maypures; Brtiteni des Maravitains. - Titi de L'Orénoeve, Simia sciurea, var., Humb., loc.cit.-Pithesc. Sä̈mil, var., Less., Species.

Car. Pelage d'un jaune doré. Deux lunules noirâtres sur la tête.

Hab. Les forêts de l'Orénoque.

Obs. Espèce encore imparfaitement connue. 
4. S. елтоморнаGE, S. entomophagus.

Syn. Calitithrix extomophagus, d'Orbigny, Allas de son Voyage, Mammif., pl. 4 (non encore décrit). -Pithesc. SAïmiri, var., Less., Species.

Car. Pelage d'un gris jaunâtre clair, devenant un peu plus foncé sur le dos. Les avant-bras et les quatre mains, jaunes. La nuque et le dessus de la tête, noirs (état adulte) ou noirâtres (jeune âge).

$H a b$. Guarayos, où l'espèce a été découverte par M. d'Orbigny.

II. CALlitriche, C.LLITRIX.

Synonymie.

Caluitricue, Calliturix, Geoff. S.-H. (1812); Desmar.; Less., Compl. de Buff.; Jard., et presque tous les auteurs.

Sagouiv, Sagunus 1. . . . L Less., Mamuel (1827); Species et Noup. tabl.

1 Ce nom aurait l'antériorité d'un grand nombre d'années, s'il était vrai, comme on l'a dit, qüll fût, dans la classification de Lacépède, la dénomination générique des Callitriches. Mais le genre Sagovin, Sagoin (et non Saguinus) de ce célèbre zoologiste n'est point le genre que nous appelons aujourd'hui Callitriche. Il a pour type, non un Callitriche (aucune espèce de ce genre n'était alors connue), mais l'Ouistiti ordinaire, Hapale jacchus, que Lacépède désigne sous le nom de Sagouin jacchus. Voyez Tableaux des divisions des Mammifères, in-4. Paris, an VII (1799). On trouve ces tableaux, réimprimés en I'an IX (1801), à la fin du troisième volume des Mémoircs de l'Instiut, elasse des sciences. 
Avant le travail de mon père, la plupart des Callitriches alors connus (et le nombre en était fort petit) étaient placés parmi les Cebus, et non parmi les Callithrix d'Erxleben.

\section{Caracléristique.}

Formes assez légères; membres allongés, les postérieurs plus que les antérieurs; pouces médiocrement allongés, les antérieurs i peine opposables. - Onglesdes pouces postérieurs aplatis; ceux des pouces antérieurs concaves, les autres reployés en gouttière. - Queue longue, grêle, entièrement velue, à peine prenante.

Tête petite, déprimće; les oreilles à peu de distance de l'occiput. - Face courte.

Yeux volumineux, séparés l'un de l'autre en avant par un intervalle assez grand, se rapprochant davantage en arrière, où ils sont, d'ailleurs, séparés (comme à l'ordinaire, mais non comme chez les Saïmiris) par une cloison complétement osseuse.-Conques auriculaires assez grandes, de forme simple.-Narines elliptiques, latérales, sćparées par un large intervalle. - Pelage très-long et très-fourni, en grande partie composé de poils annelés.

A chaque mâchoire, toutes les dents contiguës les umes aux autres; et la série continue qui résulte de cetie disposition, représentant une demi-ellipse trèsrégulière. Canines courtes et épaisses. Molaires trèslarges, à tubercules presque tous mousses : les mâchelières supérieures presque aussi étendues, et les 
inférieures plus ctendies d'avant on arrière que dans le sens transversal; dernière mâchelière de chaque mâchoire bien développée.

Taille inférieu:e à la taille moyenne des Singes.

Le Callitriche donacophile de M. d'Orbigny, figuré par lui dans l'Atlas de son Voyage, est la seule espèce nouvelle que je connaisse, et elle doit être prochainement décrite avec détail par le savant voyageur qui l'a découverte. Je dois m’abstenir d'anticiper ici sur le travail de M. d'Orbigny; et les matériaux nouveaux que je possede à l'cuard des autres espèces, ne sont pas assez nombreux pour que je puisse utilement en reprendre ici l'histoire.

Je passe donc immeriatement an woisieme des genres dont la comparaison et l'eude font lobjet de ce travail.

III. NYCTIPITHEQUE, NYCTIPITHECHUS.

S) nonymie.

Aore. . . . . . . . . Humboldt (1811).

Aotus....... Illig. $\left(18_{11}\right)$; Geoff. S.Hil., Tabl. des Quadr.; Desmar.

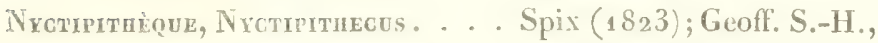
Cours; Less., Compl. de Buff., Species et Nouv. labl.; Is. Geoff., Cour's. Noctuone, Nocthoиа....... Fréd. Cuv. (1824); Less., Man. G."Cuy., Rigne an., $2^{\circ}$ éd.

Aotes........ Jard. (1833), Monk., Synops. 


\section{Caractéristique.}

Formes assez légères. Membres postérieurs beaucoup plus longs que les antérjeurs. Pouces médiocrement allongés.

Tête arrondie; front très-peu développé. Face courte, très-large.

Yeux énormes, occupant la plus grande partie de la face, très-rapprochés l'un de l'autre, surtout en arrière, où la cloison inter-orbitaire, partout osseuse, est extrêmement mince. - Conques auriculaires petites.- Narines ovalaires, rapprochées l'une de l'autre, s'ouvrant obliquement sur les côtés et au-dessous du nez ${ }^{1}$. Pelage long et bien fourni, an partie compose de poils annelés.

Incisives rangées sur une ligne courbe : les latérales supérieures séparćes par un intervalle assez étendu des canines, qui sont (chez les adultes) longues et carénées. Molaires à couronme peu étendue, à peu près aussi longues que larges, à úbercules les uns mousses, les autres aigus; dernière molaire, à chaque mâchoire, moins développée que les précédentes.

Taille tres-informe a la taille mo y mue des Singes

\$Cette disposition est assez différente de celle qu'on observe géralement chrz les Singes amóricains. 
Espèces.

1. N. FÉLIN, N. FELINUS.

Syn. Singe de nuit a Face de cirat, N. Felinus, Spix; Geoff. St.-Hil., Cours; Less., Compl.-- Douroucouli, Nocthora trivirgata, Fr. Cuv., Mammif. (confondu par cet auteur et, d'après lui, par plusieurs autres, avec l'une des espèces suivantes ).

Car. Pelage cendré supérieurement, d'um jaune olangé inférieurement (y compris la gorge). Queur rousse dans sa première moitié environ, puis noire. Une tache médio-frontale noire, assez ćtendue, à peu près aussi large que lougue; au-dessus de chaque veil, une tache blanchâtre, et plus en dehor's, deux raies noires. Oreilles courtes.

Hab. Le Para et Mojos.

2. N. Lémurin, N. Lemurinus.

Syn. Mrco dorsulov (Singe dormeur) des habilants de la Nouvelle-Grenade.

Espèce encore inédite.

Car. Pelage d'un cendré lavé de roux supérieurement, cendré sur les flanes et la face externe des membres, d'un jaune orangé sous le ventre et sous la poiurine (mais non sous le col). Queue d'un noir plus ou moins mêlé de roux, avec la base rousse en dessous et d'un cendré roussâtre en dessus. Une tache médiofrontale noire, peu étendue, entre deux taches blan- 
chitres, et plus en dehors, deux raies noires. Oreilles ures-courtes.

Obs. Il existe dans cette espèce des varjétés chez lesquelles les couleurs présentent des nuances différentes, mais toujours la même distribution.

Hab. La Nouvalle-Grenade.

3. N. A trois bandes, $N$. trivirgatus.

Syn. Vulgairement, Cousi-cousi; Singe dormeur (Mono dormlon); Singe on Titi-tigre; Cara rayada (face rayée) des missionnaires de l'Orénoque.-Douroucouli des Indiens Maravitains.

Douroucouli, Simin trivirgata, Humb.-Aotus Trivirgatus, Geoll. St.-llil., Tabl. des Quadr.; Desmar.-Douroucouli, Aotes trivirgatus, Jard.

On vient de voir que les noms de Douroucouli, N. trivirgatus, ont été transportés par erreur au N. felinus.

Car. Pelage supérieurement d'un gris argenté, avec une ligne mélio-dorsale brune, et les parties inférienres d'un jame orangé. Quene de même couleur que le dos, à l'exception de son extrémité, qui est noire. Trois raies noiràtres parallèles sur le devant de la tête: une tache blanche andlessus de chaque oeil. Nez noir, avec une ligne blanche longitudinale sur le milieu. Oreilles très-courtes.

Hab. Les forêts du Cassiguiare. 
4. N. CRIARD, N. vociferais.

Syn. Caraï des Indiens.

N. vociferans, Spix; Geoff. St.-Hil, Cours; Less., Compl. de Buff.

Car. Pelage généralement brun, les parties infériemres d'une nuance plus pâle que les supérieures. Queue d'un roux ferruginenx dans la premicie moitié,

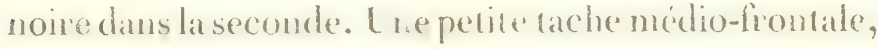
d'un noir brunàtre, une lache triangulaire jaunâtre au-dessus de chaque oeil, ef plus en dehor's, une bande noirâtre très-étroite. Oreilles très-courtes.

Hab. Les forêts de Tabatinga, au Brésil, à peu de distance des frontières du Pérou.

Obs. Cette espèce est encore très-imparfaitement connue ${ }^{1}$.

\& Je me borne à mentionner ici pour mémoire le Miriquouina d'Azara, qui est encore beaucoup plus imparfaitement connu, et que l'on ne saurait introduire dans le Systema jusqu'à ce qu'ou possède sur lui des notions plus précises. Voyez l'extrait que j’ai donné plus haut de la deseription d'Azara. 



\section{SECONDE SECTION.}

\section{CARNASSIERS.}

Les espèces dont j’ai à m'occuper dans ce travail, appartiennent totites it celte grande famille qui présente par excellence le type des carnassiers, et qui s'etend des Ours anx Tigres en passant des preminess à ceux-ci, par une longue série de genres de plus en plus carnivores, et en même temps de plus en plus complétement digitigrades. Sans entrer ici dans la discussion de rapports dont je me suis occupe il y a quelques mois dans un autre travail ${ }^{1}$, je me bornerai à liire connaitre les especes des genres Ours, Raton, Moufette et Lynx, qu'a rapportés l'expédition de la Vénus.

Outre les carnassiers qui viennent d'être mentionués, les naturalistes de l'expédition se sont procuré à l'ìle San-Lorenzo, près de Lima, un beau cràne d'OIarie, que M. de Blainville a figuré dans son Ostéographie ${ }^{1}$, et sur lequel il serait superflu de revenir ici.

Je diviserai cette seconde section en deux parties,

1 Voyez les articles Carnassiers et Carnivores du Dictionnairc universeld'histoire naturelle, t. III, p. 175 et 177 (1842).

2 Allas du septicme fascicule, pl. VI. 
l'une comprenant trois espèces, l'Ours brun, le Raton laveur et la Moufette mésomèle, tontes trois drija dablies dans la science, of sme lesquelles il sulima de passer rapidement.

La seconde partie aura pour sujet la description d'un Felis du groupe des Lynx, et à l'occasion de ce Carnassier, quelques remarques sur plusieurs de ses congénères. 


\section{PREMIERE PARTIE.}

Description de c'Ouns eruv, variété du Kautschatka, du Raton uavedr, Variété mexicaine, et de la hlodfette mésonèle.

\$I. Description de: l'ouns buv, Ursus anclos, rrriété du Kamtschatia.

Cet Ours, ramené vivant par l'exprelition, el existant encore aujourdthui à la Ménagerie du Huscum, a été pris en septruble 1837 , a l'extrémile meridionale du Kamtschatka, pres de Potropawhwrko, par j3.3 de'grés environ de latilude et 157 de longitude. L'animal était au bord de la mer, mangeant um saumon, lorsqu'il fut apercu et poursuivi. Il élait fort jeune alors: car sa longueur totale n'était que de 60 it 70 centimètres. A son arrivée en France, il y a précisément quatre ans, il avait déjà un mètre et demi de long, et il a encore grandi à la ménagerie. Aujourl'hui sa hauteur au garrot dépisse un mètre, et sa longueur totale est de $1 \mathrm{~S}$ décimètres: la tête seule, à partir du milieu de l'intervalle qui sépare les deux oreilles jusqu'au bout du museau, n'a pas moins de 4 décimètres.

Cette taille est considérable sans doute; mais ne dépasse pas les dimensions que l'on a souvent observées, non-seulement chez des Ours do Sibcrie, mais 
aussi chéz des Ours bruns d'Europe, et plus spécialement, de l'Europe septentrionale.

Le pelage est d'un brum très-foncé qui s'éclaircit un peu sur le museau et sous la gorge, et qui, au contraire, devient plus foncé encore, et même passe au noir sur la partie postérieure du dos et des flanes et sur la croupe. Les quatre membres sont de même noirs. Le mutle est noirâtre, et la muqueuse de la cavité buccale est généralement d'un rose violacé livide. Les yeux

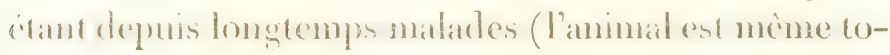
talement aveugle), je ne puis indiquer la couleur de l'iris.

Les poils sont longs sur le corps, et très-longs derrière les membres et sur le garrot, sans toutefois qu'il existe sur celui-ci celte toufle épaisse de poils excessivement longs que l'on a observée chez d'autres Ours. Sur le devant des membres, les poils sont comparativement très-courts, et ils sont peu longs sur les oreilles.

Les ongles, qui sont noirâlres, semblent plus comprimés et sont plus allongés qu'on ne les trouve d'ordinaire. Mais il faut faire ici la part de l'influence de la captivité et des habitudes paresseuses de l'animal qui, renfermé dans une cage, et passant la plus grande partie du jour dans le repos, use fort peu les extrémités de ses ongles.

D'après quelques renseignements qu'a bien voulu me donner, en l'absence de M. Néboux ${ }^{1}$, l'un de

1 M. Néboux est reparti peu de temps après l'arrivée de la Vénts en France pour une autre expédition lointaine, qu'il saura, sans nul doute, faire tourner aussi au profit de la science. 
ses collaborateurs, M le docteur Guéneau de Mussy, l'Ours du Kamtschatka était déjà brun lorsqu'on le prit, et non gris, comme le sont les jeunes de l'Ursus ferox de l'Amérique du Nord, et aussi les jeunes Ours des Pyrénées. J'ignore s’il avail de chaque côté, sur la partie latérale du col, la bande transversale blanchatre que chacun sait exister chez les jemes Ours européens, et que M. Frédéric Cuvier a retrouvée beaucoup plus marquée et plus large chez un Ours du Nord de l'Asie, considéré par lui, pour cette raison même, comme appartenant à une espèce distincte, et nommé Ursus collaris. Soit que cette bande n'ait jamais existé chez notre individu, soit qu'elle se soit graduellement effacée, comme il arrive le plus souvent chez les Ours d'Europe, à mesure qu'ils vieillissent, je n'ai pu en apercevoir la moindre trace.

La description que je viens de donner et surtout la figure, dessinée sur le vivant par $\mathbf{M}$. Werner, qui fait partie de notre Atlas ${ }^{1}$, font suffisamment connaître l'Ours ramené du Kamtschatka par la Vénus.

'Voy. la pl. IV. - Dans la planche suivante, j'ai fait représenter un beau squelette de l'U. ferox des zoologistes américains, trouvé aux environs de Monterey en Californie, et rapporté par M. Néboux. Ce squelette a déjà été figuré par M. de Blainville dans son Ostéographie (loc. cit. pl. II); mais il présente trop d'intérêt pour que j'aie pu ne pas lui donner place dans l'aulas clestiné à représenter les principaux objets des collections de la $V \dot{i}-$ nus. En effet, "c'est peut-être, dit M. de Blainville, le seul « exemple que la science possède d'un squelette d'Ours ct de grand "Carnassier parvenu à tout sou développenent a l'élat sauvage." Voyez le hunueme fascicule de l'Ostéographic. 
Elles pourront foumir par la suite quelques éléments pour la détermination des variétés que subit l'Ursus arctos dans les diverses localites où il se trouve; variétés dont on s'est hàté d'ériger plusieurs en espèces distinctes, caractérisées par quelques légères et insignifiantes differences de proportions ou par des différences de couleur moins importantes encore. Ces espèces me paraissent pour le moins fort douteuses. Les observations que j’ai faites sur les Our's de la Ménagerie, m'ont fourni, en ellet, de nombreuses preuves que, non-seulement le pelage des Ours peut varier beaucoup d'une saison à l'autre; mais qu'il présente en outre, d'un sujet à l'autre, des diversités individuelles extrêmement marquées. Ainsi tandis que j’ai vu, dans l'un des fossés de la Ménagerie, un Ours conserver en toute saison la même coloration (brun fauve clair), la plupart de nos individus ont présenté des changements très-notables, le pelage devenant plus foncé l'hiver : el encore n'est-ce pas d'une manière régulière et constante. Je puis citcr pour exemple un individu qui, après aroirété brun pendant deux ou trois années, est devenu presque aussi noir que l'U. americanus, et surtout deux individus qui, nés jumenux, tous deux máles, élevés ensemble sans jamais se quitter, et partageant constamment la même nourriture, n'ont été semblables l'un à l'antre que dans leur première jeunesse. Celte époque passée, l'un d'eux a été tantôt d'un brun-roux clair, tantôt d'un brun médiocrement foncé : l'autre tantôt d'un brun foncé, tanfot noir et tel que l'Ours noir d'Europe est décrit par 
les auteurs qui croient devoir admettre celte trèsdouteuse espèce.

\$I.-Description du Raton laveur, Procyon lotor, sarièlé mexicaine.

On n'a connu pendant longtemps, ou du moins on n'a admis dans le Systema, que deux espèces de Ratons, le Laveur, Procy on lotor, de l'Amérique septentronale, et le Crabier, Pr. cancrivorus, de l'Amérique méridionale. Ces deux espèces étaient aussi distinctes par leurs caractères zoologiques, que différentes par leurs patries. Le genre Procyon fiasait ainsi me exception tranchée à un fait général que j’ai signale, il y a plusieurs années, à l'égard des mammifères américains, et qui, malheureusement pour les zoologistes nomenclateurs, n'est que trop invarjablement vrai, savoir : la modification graduelle, et par nuances presque insensibles, des types génériques qui se trouvent répandus à la fois sur une grande partie du continent américain, et par suite l'extrême difficulté, pour ne pas dire plus, d'établir, dans les genres américains, des espèces tranchées et susceptibles d'être nettement et rigoureusement caractérisées. C'est ainsi que, depuis tant d'annces, les classificateurs s'efforcent, sans pouvoir jamais y réussir, soil de donner une détermination spécifique et satisfaisante de divers genres américains, tels que les Sapajous, les Hurleurs, les Coatis, les Moufettes, soit de définir avec quelque rigueur les espèces amricaines de di- 
vers genres cosmopolites, par exemple, parmi les carnassiers, l'Ocelot, le Chati et les autres Felis voisins de ceux-ci, el parmi les ruminants, les petits Cerfs américains. Et ici, il est à remarquer que le plus souvent les nouvelles acquisitons de la science ne font que rendre les déterminations plus difficiles et les quesiions plus obscures : preuve certaine que les zoologistes, en tout ce qui touche le grand problème de l'espèce (selon moi, encore entièrement irrésolu), sont engagés dans une fausse voie. Ils partent d'un principe inadmissible, la fixité de l'espèce ${ }^{1}$, et de délinitions, les unes inexactes, les autres vagues, hypothétiques et entièrement inapplicables aux faits: aussi n'arrivent-ils qu'à des résultats douteux, admissibles seulement à titre provisoire, et devant être nécessairement un jour modifiés ou réformés.

C'est ainsi que, près des genres que j’ai cités plus haut et qui font depuis si longtemps le désespoir ales zoologistes classificateurs, d'autres venant chaque

I "En nous élevant ici après Lamarck et M. Geoffroy Saint"Hilaire, disions-nous dans un autre travail (article Zoologic "de l'Eracyclopédie du dix-neuvième siécle), contre la doctrine de " la fixitéde l'espèce, nous n'ignorons pas que son abandon pourra " et devra entraîner l'ébranlement de l'édifice zoologique tout "entier. Mais ce danger mème, dont nous sommes le premier à " reconnaître la gravité, ne doit pas nons retenir : détruire l'er"reur, alor's même que nous ne savons pas encore exactement co: "qu’il faut mettre à la place, c’est déjà faire un premier progrès.

* Pour qui veut construire un édifice nouveau, le premier soin à "prendre, n'est-il pas de déblayer le torain des vieux matériaus "qui l'encombrent?" 
jour se placer, les découvertes des voyagenrs ajoutent bien plus encore aux embarras qu'aux richesses de la science. Ainsi, parmi les Singes, la détermination spécifique des Lagotriches, des ivychipithques, semblait, il y a peu d'années encore, aussi facile que celle des Sapajous et de Hurleurs est difficile et confuse; mais il en était ainsi, parce que les premiers de ces Singes sont aussi rares en Europe que les derniers y sont communs, et depuis quelques années le nombre des individus existant dans les collections, s'étant beaucoup augmenté, les difficultés de leur détermination se sont accrues précisément dans la mème raison.

De même les Ratons qui semblaient, il y a peu d'années encore, aussi faciles à déterminer que le sont peu les Coatis, commencent anjourd'hui a embarrasser les

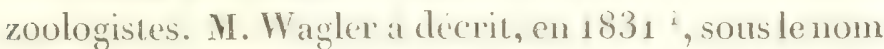
de $P$. Hernandezii, une espèce mexicaine que Hernandez paraît avoir anciennement indiquée ${ }^{2}$, et qui est intermédiaire, par ses caracteres zoologifues aussi bien que par: son habital, au $P$. lotor des Etats-Unis, et au

1 Einige Miuheilungen über Thiere Mexicos, dans l'Isis, ann. 1831, p. 514 .

2 Dans son Thesaurus historice animalium, p. 9. Voici le passage tout entier de Hernandez :

De tere maxtlaton. Genus est felis syluestris, mustellave, nam et aluro par ferè est magniudine, pilisque vestitum nigris, et promiscuè candentibus, pulvis ejus dicitur febrientibus conforre, rostrum est longum, auricula parva, breia crura, et cauda eisdem transversis fasciis, nigris, candidisque, quibus Topemaxtlu, ocoitucensis, cujus videtur species "ariure. 
P. cancrivorus du Brésil. De même une espèce qui habiterait les Antil les, P. brachyurus, une autre, habitant la Californie, P. Psora, ont été proposées, l'une en 1837 par M. Wiegmamn ', l'autre en 1842 par M. Gray ${ }^{2}$, en sorte que le nombre des espèces du genre Procyon se trouverait porté de deux à cinq, non compris le $P$. obscurus de M. Wiegmann ', dans lequel on ne samrait guère voir qu'une variété mélanienne, et non une espèce ou variété importante de localité, telle que les précédentes.

Le Raton qui fait le sujet de ce paragraphe, vient de Mazatlan, au nord du Mexique, et par conséquent a ćté trouvé dans une localité peu distante de la patrie du P. Hernanderii et de celle du P. Psora. Il était done à présumer que le liaton rapporté par la Vénus, appartiendrait à l'une ou à l'autre de ces espèces, si toutefois le $P$. Hernande $i i ̈$ et le $P$. Psora doivent être toutes deux admises dans le syı̀̇me. Or il n'en est rien, à en jugerselon les caractéristiques de ces deux espèces, telles qu'elles ont été donnćes par M.M. Wiegmann et Gray. Le Raton de la $V$ énus a de nombreux rapports avec l'une et avec l'autre; mais il s'en distingue en mème temps par plusieurs caractères. Au contraire, il a les affinités les plus intimes avec le $P$. lotor, dont je le

1 Voyez Archio für Naturgeschiche de Wirgmann, 1837 , part. IV. Ce mémoire a été reproduit daus lis sinnals of natural history" de Jardine, t. 1, p. 132.

- Dans les Annals déjà citcies de Jardine, t. X, p. 261 (décembre 4842$)$.

3 Loris cir. 
considère comme une simple variétć de localité, disfincte surtout par le moindre développement du pelage; modification dont il est à peine utile de signales la concordance avec les conditions du climat du Mexique, comparées à celles du climat des Etats-Unis.

La couleur générale de notre individu est le cendré lavé de fauve et liqueté de noir : la teinte fauve domine sur le col, le devant du dos et les épaules; la croupe, au contraire, tire sur le noirâtre. La face est noire et blanche. Le noir occupe la ligne médiane depuis le mufle jusqu'au niveau des yeux, et les parties latérales, dans une direction oblique de haut en bas et d'avant en arrière, depuis la ligne médiane jusqu'audessous de l'oreille. La bande noire oblique qui résulte de ce système de coloration, a une largeur moyenue de 6 centimètres : l'oeil s'y trouve compris, mais placé très-près de sa limite supéricure. Les oreilles sont entièrement blanches à leur face concave; elles sont de même blanches à letir face convexe, mais avec la base brunâtre. Derrière l'oreille, de chaque côté, une ligne noire, médiocrement distincte, se porte d'abord directement on arrière, puis se détourne en dedans presque à angle droit, et vient se perdre sur la ligne mediane vers le devant du dos.

Tous ces caractères sans aucune exception, et sans aucune dilférence appréciable, se retrouvent chez le Raton laveur des Etats-Unis, et la coloration généralé des poils, d'abord noirs, puis d'un cendré jaunàtre, enfin noirs à la pointe, est aussi la mème. Bais le pelage de notre Raton du Nexique est beaucoup plus Zoon.0;1: 
ras. Sous ce rapport, notre individu se rapproche du $P$. cancrivorus, et c'est, au reste, ce que chacun eût pu prévoir, en raison des circonstances climatologiques de l'habitat de ces animaux. Comme le $P$. lotor, notre Raton a les poils des pieds blancs; mais ces poils sont excessivement ras, et, sous ce rapport encore, notre Raton mexicain se rapproche du $P$. cancrivorus qui a, comme on le sait, le bas des membres denude, et rho lequed la région immédiatement au-dessus de la partie nue, est couverte de poils trèsras. Je dois même ajouter que chez notre individu le dessus de la patte antérieure droite est presque entièrement dénudé, et à peu près tel qu'on l'observe chez le $P$. cancrivorus. Cette analogie serait assurément fort remarquable; mais la disposition dont elle résulte est-elle naturelle it l'animal? On doil en douıer, puisqu'elle n'existe que d'un coté; et il y a lieu de supposer que l'animal ayant été préparé avec peu de soin, les poils de la patte antérieure droite sont tombés, comme il arrive généralement en pareil cas.

La quene, dont la longueur ne peut malheureusement être déterminće d'après une peau préparće, a, sur un fond gris jaunâtre, six anneaux noirs très-distincts, y compris l'anneau terminal. Le $P$. lotor des Etats-Unis n'a, le plus ordinairement, que cinq anneaux noirs distincts; mais souvent un sixième est indique, et parfois mène il est assez nettement marquc pour que la quene doive être dite à six anneaux; en sorte que, d'après la coloration elle-même de la queue, 
il y a plutôt lieu de réunir notre Raton mexicain an $P$. lotor, que de l'en séparer. Quant au $P$. cancrivorus, il a la queue notablement plus longue et ornée de sept ou huit anneaux; mais ceux de la première moitiv de la queue sont le plus ordinairement confluents et confus.

La taille de notre individu est un peu au-dessous de la taille ordinaire d'un Raton laveur. En raison de la moindre abondance el de la moindre longueur des poils, notre individu parait d'ailleur's plus svelte ou plutôt de forme moins lourde. Ses climensions sout comme il suit :

Distance du museau à l'originc de la queue. . $0^{\text {min }}, 55$

Hauteur aux épaules. . . . . . . . . 0 0, 25

Longueur de la queue. ......... o, 30

Il reste à comparer notre individlu aux espèces qui ont été décrites dans ces derniers temps, et quelques lignes me suffront pour cette comparaison. Aucune difficulté de s'élève en premier lieu, ni à l'égard du $P$. brachyurus, ni à l'égard du $P$. obscurus, en supposant qu'il doive être admis comme espèce distincte; les noms de ces deux Ratons suflisent pour rappeler leurs caractères distinctifs. Le. $\boldsymbol{P}$. Hernandezii est, de même, très-distinct, s'il est vrai qu'il ait les pieds bruns, et non blancs, ainsi qu'on l'admet dans toutes les caractéristiques: Maniculis ac podariis fuscis, dit M. Wagler; pedibus fuscis, dit M. Wiegmann. Enfin, le $P$. Psora est d'une autre couleur, d'après la carac- 
téristique donnée par M. Gray: Yellowish brown, dic cet auteur, and gray, grisled; face, temples, side of necti, chest, belly and sides of body dirty yellow; forchead, cheets under the ejes, ench side of the throat and back of the ears dark blaclish brown.

Brappelous du reste, en terminant, combien sont frćquentes ot remarguables les variétré individuelles chez les Ratons, et particulièrement chez le $P$. lotor, le mieux connu de tons. Parmi les individus que possède le Muséum, les uns offrent l'état normal, et, par conséquent, sont gris; d'autres, plus ou moins complétement albinos, soit jatmes ou blanes; d'autres, aflectés au contraire de mélanisme ì des degrés divers, sont gris-brum, bruns ou noirs. Fin présence de toutes ces variétés que présente le $P$. lotor anx Litats-Unis, et après avoir vu qu'il existe, au Mexique, un Raton qu'il semble impossible de séparer spécifiquenen du $P$. lolor, on est fondé à se demander si le P. Hernandezii, qui est aussi du Mexique, et le P. Psora, qui habite la Californie, sont bien des espèces distinctes, ou si ce ne seraient aussi que de simples rariétés locales ou individuclles. C'est une question que je ne puis d'ailleurs que poser, et dont la solution ne peut ètre obtenue tant que le P. Psora et le P. Hernandesii resteront comms seulement par l'examen extérienr d'un ou de quelques individus. 
SIII. - Description de la Moufette mésomèle, Mephitis mesomelas, Lichtenst.

Dans son rapport à l'Académie des sciences, sur les résultats zoologiques de l'expédition de la Vénus ", M. de Blainville a signalć, comme un fait fort intéressant pour la géographie, l'existence à la Californie du genre Noufette, si commun dans l'Amérique du Sud, et déjà connu aussi dans plusieurs parties de l'Amérique du Nord. Ce fait a été constaté par M. Néboux, qui, en eflet, s'est procuré en Californie, à Monterey, une Mloufette adulte. Examince avec soin, cette

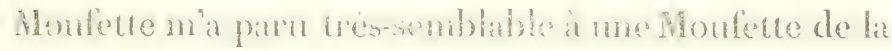
Louisiane, que M. Lichstenstein a décrite, en 1827 , dans sa Darstellung neuer Sceugethiere ${ }^{2}$, et de nouveau, en 1838, dans un mémoire special ${ }^{3}$.

Cette Moufette de la Louisiane a recu du célèbre

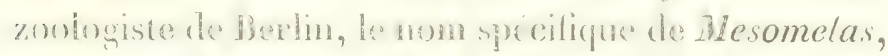
et est ainsi caractévisće par lui :

Plantis pilosis. Lineẩ angustâ medîa a rostro ad verticem; maculà nuchali antic̀̀ truncatâ, posticè bipartitî in vittam duplicem, per latera dorsi ct caude continuatam.

Celte caractéristique est parfaitement applicable

1 Comptes rendus de l'Acarlemic des sciences, t. XI, p. 33פ.

2 Berlin, in-fol., 1837 .

"Ucber die Gatlung IIephitis, eine akademische Abhandlung. Ce mémoire a étŕ publice à part (in-4, Berlin, 1838), et inséré dans le Recueil de l'Acartémie de Berlin. 
aussi bien à notre individu qu'à celui qu’a décrit M. Lichtenstein. Il existe toutefois, à quelques égards. entre l'un et l'autre des différences que je dois indiquer ici, et qu’il m'a paru nécessaire de faire connaître aussi par une fignre.

Les parties inférieures et latérales sont, chez notre individu, d'un noir profond, ainsi que le milien du dos, dans un espace triangulaire compris entre deux raies blanches obliques, partant de chaque côté de la base de la queue, et venant se réunir sur la ligne médiane entre les deux épaules. Sur l'occiput et la nuque est une large tache blanche, ayant la forme d'un triangle équilatéral dont la base serait en avant, et dont le sommet viendrait en arrière se confondre avec la ligne blanche du dos. Tout ce qui est en avant du triangle blanc occipito-cervical, est noir, sauf une trèspetite ligne blanche médiane, commencant à 2 centimètres de la tache blanehe plus haut décite, et finissant à 2 centimètres du mufle. Celte petite raie, fort étroite et tout-à-fait linéaire, n’a que 3 centimètres, encore estelle interrompue vers son milieu par des poils noirs. C'est cette ligne qui correspond à la linea angusta media a rostro ad verticem, mentionnée par M. Lichtenstein comme l'un des caractères de l'espèce; mais il est à remarquer que si elle la représente exactement par sa direction, elle est du moins beaucoup plus courte et plus étroite encore. Et même, comme elle est interrompue vers son milieu, ainsi que je l'ai dit, il y a tout lieu de présumer qu'elle disparait plus ou moins complétement chez certains individus. 
La queue, remarquable par la longueur considerable de ses poils, est mélangée de noir et de blanc. Sauf quelques pinesanx entièrement blanes, les poils de cefte partie sont blancs dans leur première portion, et noirs dans la seconde. La zone blanche des poils se montre partout à l'extérieur à travers le noir, à cause de la divergence des poils de la queue qui même est assez rógulièrement distique sur sa face inférieure ou postérieure.

Les poils sont abondants et assez longs sur le corps. Leur longueur est d'environ 4 centimètres sur la partie antérieure du dos, et de 6 et même 7 sur la croupe et vers la base de la queue. Quant aux poils de la queue, ils ont près d'un décimètre de long vers l'origine et vers l'extrémité de la queue, et jusqu'à 15 cenlimètres dans sa région moyenne.

Les dimensions générales sont les suivantes:

Distance du museau à l'origine de la queue. . $0^{\mathrm{m}}, 42$. Hauteur aux épaules. . . . . . . . 0, 12 .

Longueur de la queue, non compris les poils qui la terminent. . . . . . . . o, 25 .

Longueur de la queue, les poils compris. . . 0,32 .

Dans son mémoire sur le genre Mephitis, M. Lichtenstein a exprimé le regret de n'avoir pu examiner la dentition de la $\boldsymbol{N}$. mesomelas. On verra, par les figures du crâne et des dents que j'ai jointes à la figure de l'ensemble de l'animal, que cette espèce a bien les caractères des véritables Mephitis, et non ceux du groupe auquel M. Lichtenstein a domé le 
nom de Thiosmus, el qui se distingue par l'existence de trois molaires seulement à la màchoire supérieure ${ }^{1}$.

1 Molares suprà utrinsecius tres, dit M. Lichtenstein dans la caractéristique de ce groupe... Rhinarium proboscideum, naribus anticis et inferis. Auxicule orbiculares vix emergentes. Plante latiores dicmudalce. A ces caractères s'opposent les suivants, chroz les véritalbles Moufettes : Molares suprè utrinsecuis quatuor.... Rhinarium prominulum naribus lateralibus. Auricule ampliores

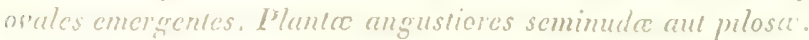




\section{SECONDE PAR'TL ${ }^{1}$.}

Description do CHAT BAI et du CHAT ALBESCENT; ext

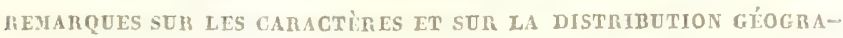
PHIQUE DE PLUSIEURS AUTHES CHATS.

Quel que soit le point de vue auquel on se place mur rudier la distribution géonaphique des mammifères à la surface du globe, soit que l'on considère cette étude comme étant de nature à jeter quelques lumières sur la formation des diverses parties di monde, ainsi que le pensait Zimmerman, soil qu'on demante anx faits quelle nous devoile, des élements pour la solution du problime relatif a l'unfluence des agents physiques sur les formes animales, on ne peut manquer de recueillir ds's notions importantes, même pour la distinction spécifigne des animaux. On arrive" en effet, par ce moyen à former des groupes comporés d'individus assez semblables, et ces groupes une fois établis, les diagnoses différentielles des espèces

1 Cette seconde partic est rédigée par M. le docteur Pucheran, attaché aux galeries de zoologie du Muséum, et dont la collaboration m'est et me devient chaque jour de plus en plus précieuse dans le soin et le classement des collections dont la direction m'est confiée. M. Pucheran, ayant fait depuis plusieurs mois une étude spéciale du groupe des Felis, je ne pouvais mieux faire que de lui confier celle partie du travail. I. G. S.-H. 
qui en forment l'ensemble, deviennent plus faciles à constater el à déterminer.

De la mêmemain qui tracait le principe de la spécialité, soit générique, soit spécifique des animanx qui habitent les latitudes australes des deux continents, Buffon esyuissait les cararéres diflerentiels qui ćtablissent ume ligne de démarcation si facile à saisir entre les Singes américains et ceux qui, dans l'ancien continent, habitent l'Afrique, l'Asie et les archipels qui en dépendent. Ln agissant ainsi, Bulfon ajoutait évidemment l'exem. ple au précepte, et faisant en quelque sorte descendre le principe qu'il avait etabli du róle speculatif qui semblait uniquement pouvoir lui être attribué, il en faisait l'application la plus ingénieuse à la distinction d'animaux que Linné lui-même avait réunis dans son genre Simia; prouvant, par cet exemple, que si les généralisations satisfont l'esprit en lui faisant considérer les faits d'un point de vue plus large et plus vaste, elles ne sont pas moins utiles à la science par la manière dont elles simplifient la solution des problèmes qui composent son domaine.

Aussi tous les zoologistes qui ont suivi Buffon, ontils généralement sanctionné, par leur approbation, les vues de cet homme célèbre. Nous dirons même que la distribution grographique des races humaines conconcorde parfaitement avec les lois de distribution géographique que Buffon a chablies. C'est la mème race qui, dans les deux continents, en Asie et en Europe, comme en Amérique, habite les latitudes borćales. Dans les latitudes australes, au contraire, qui ne saic 
que les pruplades do l'A merigune du sud sont différentes de celles du sud de l'Afrique? Qui ne sait encore que les nations qui peuplent l'Asie, les divers archipels de l'Océanie et le vaste continent de la Nouvelle-Ilollande, ne sont point les mèmes que celles qui peuplent le sud de l'Afrique et de l'Amórique?

Mais si, d'une part, comparés aux animaux de l'ancien continent, les animaux américains en diffèrent comme espèces, on ne peut s'empêcher de convenir, d'autre part, qu'ils oflirent des ripports d'ensemble et de forme qui permettent d'atublir leur parallélisme réciproque. Ainsi, dans la famille des Singes, les Alcles du nouveau continent correspondent aux Semnopithèques de l'ancien, les Ilurleurs aux Macaques; de facon que les Animaux des deux mondes, comparés soil entre eux, soit avec leurs congenères des mêmes localités, offent des ty pes de forme totalement analogues.

Daus les types divers de formes et de coloration que nous offrent les espèces du genre Felis, on retrouve ce même parallélisme de création des deux mondes. Ansi, parmi les espèces unicolores à grande taille, le Couguar, en Amérique, correspond au Lion de l'ancien continent; dans les espèces à taille inférieure, l'Eyra et le Jaguarondi, correspondent au $F \boldsymbol{e}$ lis Temminkii de MII. Vigors et Horsfield, et au Felis moormensis de M. Hodysun. Ainsi, encore dans les deux continents, on retrouve des espèces à pelage couvert de taches en rose; en Amérique, le Jaguar, et dans l'ancien continent, les divers types de forme que l'on rapporte à la Panthère et au Léopard. Aussi, 
en décrivant dans le Zoological journal, le Felis macrocelis de M. Tenminck, M. Horsfield n'hésita point à dire qu'il représentait dans l'ancien continent les Chats at taches ocellées du continent américain.

En général, comparées ì leurs analogues de l'ancien continent, les espèces du genre Felis propres au continent américain, s'en distinguent par un prolongement caudal moins allongé. Que ce fait soit vrai pour les Chats annéricairs à pelage simplement tacheté, ceci ne doit pas nous surprendre; car on observe ce caractère de brièveté de la queue dans la presque totalité des espèces de l'ancien continent, que la coloration de leur robe rapproche du Serval, telles que les Felis javana el sumatrana de M. Horsfield, le F. rubiginosa de M. Isidore Geolfroy St.-Hilaire, le Felis viverrinus de M. Bennetl, et la plupart des individus qu'ont décrit dans ces derniers temps MII. Gray ${ }^{1}$, Elliot ${ }^{2}$, Hodgson ${ }^{3}$, Ogillsy ${ }^{4}$, Lesson ${ }^{5}$, Jardine ${ }^{6}$, et Waterhouse ${ }^{7}$. Mais on sait que le Jaguar, compare?

2Fclis neglecta, Amm of nat. hist., t. I, 1838 , p. 27. - Felis chincnsis, daus Loudou's magazine, t. I, 1837 , p. 577 .

2 Felis IV agati, Journal de Madras, $\mathbf{n}^{\circ} 24$.

Felis viverriceps, dans le Journ. of asiat. soe. of Beng., t. V, Iro part., p. 232 .

4 Felis scrwalina, dans les Proceed. of the zool. society de Londres, 1839, p. 94 .

‘ Felis senegalensis, dans l'Instilut, 1834 .

- Felis himalaicus, dans the nalur. library, t. II, p. 230. Le felis servalina du même naturaliste paraît faire exception, en supposant que ce soit bien une espèce; car il a une queue trèslongue.

7 Folis mutilus, dans les Proced. 18.42, p. 180. 
a la Panthère, se distingne par une quene plus courte; caractère qui, indépendamment de ceux fournis par la forme des taches, permet de reconnaître facilement ces deuxe spèces.

Nous croyons être dans le vrai, en disant qu'il en est du Couguar, de l'Eyra et du Jaguarondi comparés sous ce point de vue au Lion, au Felis Temminckï el au Felis moormensis comme du Jaguar comparé à la Panthère.

Au reste, tous ces Chats à taches ocellées qui paraissent habiter presque uniquement le continent américain, ne peuvent laisser le moindre doute à ce sujet. S'il est varai que le Felis macrocelis de Sumatra représente, dans l'ancien monde, les Ocelots du nouveau, l'allongement de sa queue est un caractère bien suffisant pour l'en distinguer avec facilité. Enfin c'est tout au plus si, dans le Felis macroura de $\mathbf{H}$. de Wied ', on trouve une espèce qui représente à un frible degré, par une queue un peu plus allongée que ne le sont celles de ses congénères, les divers Chats qui ont été décrits sous le nom de Felis pardus, Felis leopardus, Felis irbis et Felis Nimr', et dont les caractères distinctifs sont déterminés d'une manière si confuse. Quant au Chati, aux diverses variétés d'Ocelor, décrites par M. Griffith d'après les observations du colonel Hamilton Smith, et dont M. Fischer a fait des espèces, elles confirment en tout point les domées

I Nous ue parlons point du Felis clegans de M. Lesson par le motif bien simple que nous croyons que c'est la même espèe que: le Chat de M. de: Wied. 
différentielles qua nous venous de briexement esquisser. Un individu que nous possédons présentement dans la collection de mammalogie du Muséum, ne fait pas non plus exception sous ce rapport.

Dans ce Felis, le fond du pelage est blanchâtre, lavé de fauve sur le front et la tête, sur le dos du cou, sur la grande tache de l'épaule el au centre des petites taches qui se voient sur la face externe des membres antérieurs.

A l'angle interne de l'ocil se voit une petite tache moire, pleine, verticale et allongce, du centre de laquelle partent quelques soies, et immédiatement audessus, séparée de la première par un intervalle blanc fauve, s'en trouve une autre plus large, également verícale et dont l'extrémité supérieure est dirigée en dedans. Au-dessus de cette seconde tache, et séparće d'elle par un intervalle igalement fauve, commence une raie noire qui se dirige en arrière et, au niveau du bord interne de l'oreille, se dirige en dedans; cette partie transversale occupe sur le front l'espace d'un centimètre.

Ces deux raies interceptent entre elles un espace lauve blanchâtre, occupé par des taches noires, les unes verticalement, les autres transversalement dirigées: sur la portion du front, qui sépare les unes des autres les deux taches superposées situćes à l'angle de l'oeil, ce ne sont plus que des mouchetures. Disons cependant qu'en dedans de la tache intra-oculaire la plus antérieure se trouve, ì droite et à gauche, une tache noire allongée. 
L'occiput est occupé par sept lignes noires, longiudinalement dirigées. Les deux plus externes naissent à gauche d'une ligne de même couleur qui commence à quelques millimètres en avant de l'oreille, en arvière de la courbe que forme pour s'inflechir en dedans la ligne noire qui occupe le front et dont nous avons déjà parlé. Elle cotoie ensuite l'oreille à sa base, pour se diviser en deux raies qui descendent le long du cou, et se réunissent de nouveau, interceptant une tache fauve qui infériemrement, au-dessus de la ligne transversale de réunion des deux raies qui la bornent, présente deux mouchetures noires. A droite, elles naissent en haut isolément, de sorte que la longue tache qu'elles forment est ouverte supérieurement. Celle qui occupe la partie médiane est une raie pleine, naissant plus en arrière que toutes les autres, ì 13 centimètres $\frac{1}{2}$ de la pointe du nez; elle s'étend sur la région médiane du dos, jusqu’à la réunion du tiers moyen avec le tiers antérieur de l'espace compris entre le bout du nez et l'origine de la queue : c'est là qu'elle cesse. Quant aux deux raies qui, toujours sur la partie supérieure du cou, sont. situées à droite et à gauche de la ligne médiane, elles naissent plus en avant que toutes leurs congénères, au niveau du bord antérieur de l'oreille, à 11 centimètres environ du bout du nez. Elles se dirigent d'abord verticalement en arrière, puis en dehors et ensuite reprennent leur direction première. A droite et à gauche, chacune d'entre elles se divise, interceptant un espace fauve et descendant le long du cou, 
elles se séparent de nouvean, de facon que l'espace qu'elles interceptent est ouvert inférieurement; mais, dans un plus grand espace à hroite qu'à gauche, les deux raies venant plus au contact sur cette dernière partie du con.

Sur le milieu du dos, règne une série de taches allongées, lont l'extrémité de la plus antérieure commence a 47 centimètres environ de la racine de la quene. Les deux prenières sont parfaitement isolées: les autres tendent de plus en plus à se constituer en ligne continue, et cette fusion est d'autant plus complète que l'on se rapproche plus de l'origine: de la queue. Dans l'endroit oì deux taches tendent it se confondre, la ligne médiane présente un rétrécissement dû à la moins grande largeur de la tache qui occupe cet espace. Toutes ces taches sont d'un noir' parfait : les poils qui les forment sont grisatres à leur racine, puis brunâtes et noirs dans le reste de lemr étendue. Cà et là, on aperecoit dans leur intérieur on dans leurs points de fusion, quelques poils ì pointe fauve; chez ces derniers, qui se présentent surtout dans les points d'intersection des taches et qui sont des vestiges de leur séparation, un anneau fauve se trouve subjacent ì l'ammen noir de la pointe. Cet ameau fauve varie en ćtendue; il nous semble de nasure à fare présumer que dans les mues antérieurement répronvées par l'individu que nous décrivons, les poils qui se trouvent noir's dans la majeure partie de leur étendue araient été en partie de conleur faure, an moins à lenrs pointes. 
Sur les côtés de cette ligne dorsale, se trouvent deux rangcées de taches, allongées dans le sens de la longueur de l'animal, bordres de lignes noires, ouvertes pour la plupart et de couleur blanchâtre, légèrement teintes de fauve dans leur centre, les poils qui forment la partie centrale ayant leurs pointes de couleur fauve très-clair. Plus on se rapproche de la queue et plus les taches deviennent étroites : à droite, on voit que ces taches ont moins qu'à gauche de la tendance à se dessiner en bandes.

Au-dessous de ces rangées de taches, s'en trouvent deux autres totalement ocellées. La plus supérieure est bordée de lignes noires ondulées, qui à son bord postérieur ne viennent point au contact. Le centre de cette tache, qui est de couleur blanchâtre, les poils qui le composent étant de cette couleur dans leurs doux tiers supérieurs, le centre de cette tache présente des mouchetures noires; à la réunion de son tiers antérieur avec ses deux tiers postérieurs, une raic noire verticale la divise en deux parties. Elle a 23 centimètres environ dans ses dimensions longitudinales, du côté gauche; du côté droit, sa partie postérieure n'est point encore réunie à sa partie centrale. La tache qui lui est subjacente est de même forme, mais plus longue, présentant plus de mouchetures, tant à droite qu'à gauche, que celle qui lui est superposée. Des deux côtés du corps, elle va s'arc-bouter en avant, sur la bordure noire qui limite supérieurement les petites taches qui couvrent les épaules; en dehors et en arrière, elle est continuće par les taches 
allongées et imparfaitement bordées de noir, qui couvrent la face externe des cuisses et les hanches.

Au-dessous de ces deux taches s'en trouve une troisième, ocellée comme elles, occupant environ la moitié de l'espace qui sépare le membre antérieur du membre postérieur : elle est continuée en arrière par d'autres qui sont imparfitement bortées et ne communiquent point avec elle, isolées même qu'elles sont les unes des autres.

Les épaules sont occupées par de nombreuses taches et mouchetures noires, quelquefois se convertissant en lignes; une raie noire les encadre en dessus et en dessous. D'autres, de même couleur, affectant la disposition transversale, entourent les membres antérieurs et postérieurs, eu dehor's comme en dedans. Aux membres antérieurs, ces taches présentent en avant un grand nombre de poils, dont les pointes sont de couleur fauve. Les pattes sont, aux deux membres, mouchetes de noiritre; au menbre postórieur, une tache présentant cette coloration s'étend cu arrière presque jusqu'au jarret ${ }^{1}$.

L'abdomen présente un fond de couleur d'un blanc très-pur : il est occupé par des taches d'um brun chocolat, assez larges, et offrant un grand nombre de poils à pointes blanches. II en est de meme te la líce interne des membres.

I.es joues, le menton et la gorge, sont d'un blanc

'Les ongles sont comprimés, assez forts, de couleur nacrée, et plus longs en avant qu'en arriere. 
très-pur. De l'angle antérieur externe de l'oreille, part une ligne noire yui va en s'élargissant et, recevant un autre trait noir qui aboutit en avant à l'angle externe de l'oeil, rejoint sa congénère sous la mâchoire inférieure, de ficon à former à l'animal un collier complet. Au-dessous de ce collier se trouvent, sur les cotés de la ligne médiane, deux traits noirs, le premier transversalement et le second, en dehors de celui-ci, verticalement dirigés. Enfin, en avant de l'espace qui sćpare l'un de l'autre les deux membres antérieurs, se trouvent trois autres colliers, le plus supérieur bien complet, les deux autres formés de taches transversalement dirigées et isolées : ils unissent l'une à l'autre la grande plaque de taches qui couvre les épaules en dehors. Jues soies des moustaches sont les unes en entier blanches, les autres en entier brunâtres : quelques-unes sont brunatres à leurs racines et blanches dans le reste de leur étendue. En arrière d'elles, commence une ligne noire effilće qui se change, sur la joue, en une large tache de même couleur, présentant dans son centre quelques poils ì pointes fauves. Les oreilles très-larges et très-amples, offrent au dehor's un liseré noir sur le bord externe de la tache blanche qu'elles présentent dans leur centre.

La queue qui descend jusques à la plante des pieds, présente à sa base des taches noires alfectant une disposition transversale; mais, à partir de la réunion de son premier avec son second liers supérieur, ces anneaux deviennent plus distincts. Près de la pointe, it en existe deux qui ont jusqu'à 3 et 4 centimètres de 
largeur. Le fond blanc qui sépare les anneaux noirs perd de plus en plus de sa largeur, à mesure que l'on s'approche de la pointe qui offre quelques poiss blancs sur sa face inférieure, et supérieurement offre un anneau noir.

Les dimensions de cet individu monté sont les suivantes :

Longueur du corps du bout du nez à la rácine de la queue. ................. 78 cent

Longueur de la quene. . . . . . . . . . . . . . 3.1

Distance du bout du nez à l’angle interne et antérieur de lorille. . . . . . . . . . . . $11 \frac{1}{2}$

Distance du bout du nez à l'angle externe et antérieur de l'oreille. . . . . . . . . . . . 11 Distance du bout du nez à l'angle antérieur de l'cil. . . 4 Distance entre les deux oreilles. . . . . . . . . 7 La plus grande hauteur de l'oreille est de. . . . . . 7 Sa plus grande largeur de. . . . . . . . . . . 4

Nous considérons cet individu comme étant simplement une diflérence d'ige de l'uspree que M. Frédéric Cuvier a décrite sous le nom de Felis brasilionsis, en juillet ${ }_{1828}$ ( $L V H I^{\mathrm{e}}$ livraison), daus l'ouvrage de mammalogie qu'il publiait avec l'un de nos grands maîtres en zoologie et en anatomie comparée, M. le professeur Geoffroy Saint-Hilaire père. Il est bien vrai que dans la figure pleine d'exactitude que M. Werner a donnée de l'animal, les taches sont beaucoup moins ocellées que dans lindividu que nous avons décrit; mais cela provient de la circonstance suivante. M. Frédéric Cuvier n'a décrit que le côté droit 
de l'animal, et c'est aussi ce côté qu'il a fait représenter. Sur le côté gauche, en elfet, les taches sont beaucoup mieux ocellées que sur le côté droit. 'Tel est le motif qui nous fait considérer ces deux Chats comme étant la même espèce : car tous les deux se séparent des Ocelots par le fond blanchatue du pelage et du centre des taches des flanes : tous les deux ont les oreilles plus grandes, plus étalées, le museau moins large que l'Ocelot.

Mais cette espèce mérite-t-elle de conserver dans les catalogues des especes lu genre Chat, la dénomination de Felis brasiliensis, que lui a imposée M. Fr. Cuvier, croyant que l'individu qu'il décrivait avait èté amené du Brésil à Cuba et de l'lle de Cuba en France? C'était la croyance de M. Frédéric Cuvier au mois de juillet 1828 ; mais dans la seconde édition de son ouvrage, en 1834, il rectifia cette donnée, en disant que cet animal avait été amené en France de l'lle de Cuba, mais que l'on ignorait de quelle partic du continent américain il y avait été apporté. Quant it lindividu que nous avons décrit, c'était un individu du sexe mìle, originaire de l'état d'Arkansas, dans la Louisiane, et qui avait été donné à notre ménagerie par M. Trudau. lien ne nous prouve que cette espèce habite le Brésil, et comme l'application du nom cité plus haut entraine à sa suite une erreur, nous croyons légitime, jusqu’à plus ample informe, de lui substituer la dénomination de Chat albescent (Fclis alhescens), dénomination qui rappelie à la mémoire lit teinte blanchâtre du pelage. 
Mais si le continent américain, dans ses parties australes, parait surtout labité par les Fólis à taches ocellées, l'ancien continent à son tour, dans ses latitudes correspondantes, parait être la patrie à peu près exclusive ${ }^{1}$ de ces espèces de Chats, que Pallas, suivant M. de Blainville, avait désignés sous le nom de Cato-lynx, espèces qu'isolent si naturellement leu' pelage en général touffu, et leurs oreilles uniformément colorées en dehors.

Il devient évident dés-lor's que la plupart des Fólis munis de trois molaires à la machoire supérieure et ¿̀ oreilles dénuces de pinceanx de poils bien marqués, sont surtout habitants des régions australes. C'est l'inverse pour les Lynx, dont le séjour est réellement propre aux zones boréales des deux hémisphères.

C'est à cette division du genre Félis, qu'appartient le Chat bai (Felis rufa, Guld.), rapporté de Califormie par M. Néboux. Le pelage est long, touffu, doux au toucher : il est sur les flancs fauve roussâtre, teint de blanchàtre, la plupart des poils ctant de cette dernière couleur dans leur moilié la plus externe. Sur la ligne médiane du dos, règnent depuis l'intervalle de séparation des épaules, des stries noires non limitées, produites par la prédominance, dans cette ré-

I M. Gay, auquel nos collections doivent tant de richesses, nous ayant rapporté un Chat qui, par ses caractères extérieurs, nous a semblé appartenir à cette section des Cato-Iynx, on s'expliquera comment nous sommes moins aflimatifs sur le fait de la distribation géographigue des psendo-lynx. 
gion, des poils a pointe noire. La teinte roussâtre domine sur le dessus du cou', la teinte blanchâtre sur le front où se dessinent quelques lignes horizontales brunâtres : l'extérieur des membres présente la coloration fauve roussatre des flancs; elle est plus pure sur les membres postérieurs, plus lavée de blanchâtre sur les membres antérieurs. Les taches brumes noirâtres qui les couvrent sont également plus détachées en avant qu'en arrière : elles sont surtout marquées en dedans oì le pelage est blane. Le haut des membres présente en dedaus deux bandes transversales parfaitement bien tranchées et d'un noirâtre foncé. Le dessous est blanc, marqué de taches noires. La queue est blanche en dessous el noire en dessus à son extrême pointe.

Ce Chat, qui a été tué à Monterey, avait donc revêtu sa robe d'hiver. D'après la description que nous en avons donnce, il est facile de voir que si les lignes noires de la région dorsale sont mieux dessinées chez les individus dans leur pelage d'été, les taches brunes noirâtres de la face externe des membres, le bout de la queue noir en dessus et blanc à sou extrime pointe, permettent (ces caracteres sont recomaissables en toute saison), permettent, disons-nous, de différencier l'espèce des autres Lynx de ces régions. Ces parties présentent donc, chez le Felis rufá, le même caractère de permanence qui est dévolu aux taches des fesses et de la face chez certains Cerfs auxquels les saisons font éprouver des changements de coloralion. 
Les dimensions sont les suivantes:

Du bout du nez à l'origine de la queue. . . . . 59 cent.

De la quene. . . . . . . . . 11

Longueur du membre antérieur. . . . . . . . 18

Longueur du membre postérieur mesuré le long de son bord postérieur. . . . . . . . . . 22

De l'angle interne de l'œil au bout du nez. . . . . 3

Du bord interne et antérieur de l'oreille au bout du nez. $\quad 8 \frac{1}{4}$

Les dimensions de cet individu prouvent qu'il était jeune; les formes propres au cràne ne nous laisseront point d'incertitude à ce sujet.

On sait que, comparé au crâne du Felis borealis de II. 'Temminck, le crine du felis bai s'en distingue par' une saillie plus grande de la crête sagiltale, par un plus large étalement du frontal, par un cadre orbitaire plus allongé, par des arcades zygomatiques plus déjetées en dehors. Dans la face, les os nasaux sont plus étalés, plus larges, et le bord, par lequel ces pièces osseuses s'missent au frontal, est plus étendu. En outre, la ligne d'articulation des os nasaux avec le frontal, au lieu de se trouver sur la même ligne horizontale que la suture de l'articulation du frontal avec lit portion montante du maxillaire supérieur, cette articulation se trouve au-dessus de cette dernière. Sur le crîne de l'individu rapporté par la F́énus, les caractères que nous venons de tracer se trouvent réunis, mais modifiés, comme le sont les cranes de tous les jeunes animaux, par rapport it ceux de l'adulte de l'espèce it

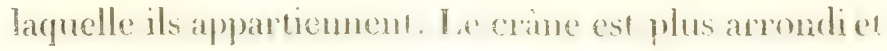


plus aplati que chez l'adulte, les sutures non encore totalement effacées n'ont point permis aux cretes d'insertion musculare d'obtenir le diveloppement qui les caractérise; les rugosités d'insertion du muscle temporal ne sont même point ébauchées; la portion de la voûte du crâne qui se trouve située derrière l'apophyse orbitaire du frontal, n'est guère plus comprimée que la partie de la région temporale qui sert de point d'attache à la racine transversale de l'apophyse zygomatique; cette apophyse zygomatique elle-mème est peu déjetée en dehors. Arec l'age les formes propres à l'adulte se dessinent et s'établissent: le cràne s'allonge et se rétrécit, les crêtes et les rugosités osseuses se dessinent et les caractères de l'instinct carnassier se révèlent par le développement des organes dévolus anx fonctions de la mastication. 


\title{
TROISIEME SECTION.
}

\author{
RONGEURS.
}

Parmi les Rongeurs rapportés par l'Expédition, trots espèces mont paru pouvoir citre utilement décrites et figurées: ce sont le Néotome de la Floride, l'Ecureuil à ventre roux, dans divers états de pelage, et une espèce nouvelle ì laquelle j’ai cru devoir domner le nom du savant chirurgien auquel est dû l'individa type, MI. le docteur Néboux.

SI. Note sur le Níotone de la Floride, Neotoma floridama, $\mathrm{S}_{A Y}$ et Ord。

On sait que le genre Néotome a été établi, en $1825^{1}$, par MII. Say et Ord, d'après un Rongeur de la liloride de l'est, précédemment therit par M. Ord" comme une espèce nouvelle du genre Rat et nommé Mus floridanus : c'est aujourd'hui le Neotoma floridana, comu non-seulement en Florjde, mais aussi dans plu-

1 Voyez le Journal of the Acad. of natural sciences of Philadelphire, 1. IV, p. 345 .

'Bullctin de lu socićté philomathique, an. $1818, p .181$. 
sieurs autres parties des Ltats-Unis, notamment dans la région qu'arrosent le Mississipi et le Missouri.

C'est cette même espèce que M. Néboux nous a rapportée de la Basse-Californie, où elle se retrouve avec la même taille, mais avec une nuance un peu plus grise. La queueest aussi un peu plus blanche inférieurement. Ces différences sont d'ailleurs sans importance; et si j'ai cruderoir revenir ici sur le Néotome, c'est, d'une part, pour constater son habitat en Californie; de l'autre, afin de mettre à profit l'occasion qui s'offrait it moi de donnex une bonne figure colorice du Néotome, représenté seulement en noir par MM. Say et Ord. Lá détermination spécifique des Rongeurs, et surtout des Muridés, présente, en effet, de telles difficultés que l'on ne doit laisser échapper aucun moyen de fixer nettement leurs caractères spécifiques. Peut-être notre figure sera-t-elle consultée utilement par les zoologistes qui chercheront à déterminer enfin d'une manière rigoureuse les rapports, naturels et, par conséquent, la véritable place d'un Rongeur que l'on trouve décrit, tantôt sous le nom Ncotoma Drummondii, tantôt sous celui de Myoxus Drummondii, et qui, en réalité, est trop imparfaitement comnu pour qu'on puisse se prononcer à son égard sans le secours de nouveaux matériaux. 
\$I. Note surles Lcurnuns rapportés par l'crpédition de la V’énus, Sciurus aureogaster, Fr. Cuv., et Sciurus Nebouxii, Is. Geoff.

On doit à l'expédition de la Vénus, et spécialement aux recherches de M. Néboux, plusieurs Ecureuils intéressants, la plupart venant de la Californie, un autre du Pérou. Les premiers se rapportent au Sciurus aureogaster de Mi. Fredúric Cuvier, bien qu'jls manquent plus ou moins complétement du caractère que rappelle ce nom; et l'on va voir, par les détails que je donnerai sur cux, combien l'on doil modifier la caractéristique ordinairement assignée à l'espèce.

Quant à l'Ecureuil du Pérou, il appartient à une espece nouvelle à laquelle je domnerai le nom du savant et zćlé médecin de la V'énus.

1. L'Écureull a ventre dorÉ, Sciurus aureogasler, Fr. Cuv.

Deux individus de cette espèce, de sexe différent, mais fort semblables l'un à l'autre, ont vecu il y a quelque temps à la ménagerie du Muséum, et c'est d'après eux que M. Frédéric Cuvier a établi l'espèce. Je citerai ici textuellement une partie de l'article qu'il lui a consacré dans son Histoire naturelle des Mammiferes de la ménagerie ${ }^{1}$.

${ }^{1}$ Septembre 1829 . Cet article est intiule: Ecurcuil de la Californic ou àvontre doré. 
"Cet Ecureuil nouveau paraît être propre aux ré"gions occidentales de l'Amérigue du Nord, depuis le "Mexique jusqu'en Californie. M. Desmarest a eu la " complaisance de me communiquer la description qu'il " avait faite au Havre d'un individu de cette espèce qui " avait été pris entre Mexico et la Vera-Cruz...

"L'Ecureuil à ventre doré, comme l'Ecureuil de la "Caroline, a toutes les parties supérieures grises, mais " un peu plus foncées; et au lieu de n'avoir que quel"parties des flancs d'une teinte fauve, il a toutes ses "parties inférieures d'un roux orangé ou doré bril"lant ... La queue est distique, et les oreilles ne sont " point surmontées par des pinceaux comme chez l'E" cureuil commun. L'iris est brun et la pupille ronde. "Cette espèce est plus grande que l'Ecureuil de la "Caroline; la longueur de son corps, du bout du mu" seau à l'origine de la queue, est de dix pouces. La " queue en a huit.

"Je proposerai de donner à cet Ecureuil, qui n'est " point encore entré dans les catalogues méthodiques, "le nom de aureogaster, à cause de la couleur de ses "parties inférieures."

C'est en 1829 quela ménagerie du Muséum avait reçu de la Californie les deux individus sujets de la description précédente. En 1831, d'autres individus, entièrement semblables, vinrent se placer près d'eux dans les galeries du Muscum. Ceux.ci faisaient partie d'une collection de mammifères et d'oiseaux du Mexique, acquise à Berlin par les soins de M. le professen T alenciennes. Ils portaient sur leurs étiquettes le nom de 
Scrurus hypoxanthus, sous lequel M. Lichstenstein avait provisoirement désigné l'espèce dans le Muscé de Berlin.

L'épithète spécilique d'hyoxanthus est évidemment lirće du même caractère que M. Frédéric Cuvier avait exprimé par le nom d'aureoguster ${ }^{1}$; et depuis MII. Frédéric Cuvier et Lichstenstein, tous les auteurs ont adopté l'un ou l'autre de ces noms, et caractérisé le Sciurus aureogaster ou hypoxanthus par la couleur rousse de ses parties inférieures.

Et cependant, il faut recomnaitre aujourd'hui que ce caractère n'est nullement constant : le ventre est, chez certains individus, d'un gris clair, n'offrant pas méme la plus légère teinte de roux. Parmi les Ecureuils rapportís de Honterey par la fénus, il en est un qui oflire. ce caractère; ct un autre, que j’ai fait représenter sur

1. Ie nom de S. hypopyrhius, que M. Wagler (Isis, 1831, p. 510 ), adonné à un Ecureuil du Mexique, a encore le mêmesens, et se rapporte au mème caractère. Voici la caractéristique du $S$. hypopyrrhus telle que la donne M. Wagler :

Cauda longissima, nigra, pilis nonnullis, omnibus versis ejus basinfuscescenti-griseo variegatis; capile, trunco pedibusque supri nigris, undique fuscescenti-sive flavido-griseo irroratis; auriculis, naso, podarits ac vibrissis atris; gestreco pedumque latcre interno forrugincis.

Je n'ai vu chez aucun de nos 5 . aureogaster tous ces caractères réunis : mais les différences sont si légères, et les Ecureuils, comme cet article même va en fournir de nouvelles preuves, sont d'ailleurs sujets ì tant de variettis, que l'existence au Mexique de deux espèces aussi voisines que le seraient le $S_{c}$. aureosaster ou Sc. hypoxanlhus, et le $S_{c}$. hypopyrrhus, doit sembler pour le moins bien douteuse. 
la même planche, a même le ventre aussi blanc qu'un Ecureuil de la Caroline. Mais celui-ci, chez leguel la nuque et la croupe sont rousses, a le dos et les membres d'un gris très-clair, et est évidemment revètu d'un pelage anomal ${ }^{1}$ : il présente à notre observation un cas d'albinisme patiel, fort remarquable sans doute, mais dont on ne peut tirer aucune conséquence relative aux caractères de l'espèce. C'est donc à l'examen du premier individu que je dois spécialement m'attacher.

Celui-ci, qui paraît mâle ${ }^{2}$, est généralement gris en dessus; mais le gris n'est pas pur, les poils, noirs à leur base, blancs à leur pointe, ayant une zone intermédiaire rousse qui se montre un peu au dehors, et jette sur l'ensemble du pelage une lógère nuance rousse. Sur la croupe et la nuque le roux devient mème dominant. Le dessus de la tête et des pattes est d'un roux tiqueté de blanc; et le tour de la bouche et tout le dessous sont d'un gris trés-clair onblane-grisitre. Les oreilles n'ont que des poils très-ras et sont grisitres. La queue, dont la base est rousse, est en-dessus d'un noir fortement nuancé de blanc, les poils ayant leur extrímité blanche sur une assez grande étendue. En des-

1 Les poils ont sensiblement la même longueur clans les parties qui ont conservé leur couleur normale et dans celles qui sont atteintes d'albinisme. Parmi les autres Ecureuils albinos que possède le Muséum, se trouve un Ecureuil d'Hudson chez lequel une partie du pelage n'est pas moins anomale par sa brièveté que par sa blancheur. J'ai mentionné déjà ce cas remarquable dans mon Histoire générale des anomalies, t. I, p. 312.

${ }^{2}$ L'état imparfait de la région sexuclle m'oblige de m'exprimer ici avee quelque doute. 
sous, la queue, qui est distique, montre très-distinctement, et pou ainsi dire disposces par bandes longiunlinales, les trois coulen's que présente chaque poil en particulier, le roux, le noir, le blanc. Ainsi, la première partie des poils forme, de chaque côté, le long de la ligne médiane, une bande rousse, limitée en dehors par une bande noire, en dehors de laquelle les extrémites des pois forment une bande blanche, d'ailleurs moins étendue el plus confuse.

Les grandes dents antérieures sont d'un jaune orangé en avant, blanches latéralement. Les moustaches sont noires.

La longueur de l'individu que je viens de décrire est d'un peu moins de trois décimètres, non compris la queue, qui a environ deux décimètres et demi.

En comparant cette description à celle de RI. Frédéric Cuvier ou à l'un des individus qui nous sont venus en 1829 et en 1831 de la Californie et du Mexique, on reconnaîtra immédiatement de nombreuses et remarquables analogies avec ceux-ci, mais aussi de notables différences. L'Ecureuil de la Ténus, en même temps qu'il manque inférieurement de la coulemr rousse qui serait caractéristique pour l'espèce selon les aua teurs, plus de roux sur les parties supérieures; et cela, non-seulement sur la croupe et la nuque où le roux domine, mais même sur le dos, où les poils ont une zone rousse dont d'autres individus ont à peine un vestige. L'Ecureuil de la Vénus ne devrait-il done pas être considéré comme une espèce voisine, mais distincte du Sciurus aurengraster ou hrpoxanthus? 
Si je n'avais comme éléments de détermination que les individus dont j'ai fait mention jusqu'à présent, l'hy pothese de deux especes fort roisines, l'une à ventre roux, l'autre à ventre gris-clair, serait fort soutenable. Mais j'ai sous les yeux plusieurs individus dont l'examen démontre d'ume manière certaine la variabilité de la coloration des parties inférieures, qui sont tantôt rousses, tantôt d'un gris clair, et tantôt variées en des proportions diverses de roux et de gris ${ }^{1}$.

Le premier individu à ventre varić que j'aie vu, est une foncle dune, en 1836, an Museum de Paris pate

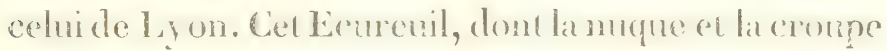
(celle-ci sur une très-grande étendue) sont roussâtres, a le ventre coloré de roux et de gris clair selon une disposition très-remarquable. Le devant de la poitrine est entièrement d'un roux beaucoup plus pâle que chez les individus de M. Frédéric Cuvier; mais, sur la partie postérieure de la poitrine et sur le ventre, le foux forme wois bandes longitudinales, deux laterates s'étendant de chaque côté, comme chez l'Ecureuil de har Caroline, de linsertion du mentre andrieur a celle

I Il est peu de genres dans lesquels les espèces soient, plus que chez les Ecureuils, sujettes à des variations indivividuclles. Tout le monde connaît les belles variétés que présentent le Sciurus vulgaris dans le nord de l'Europe et de l'Asie, et les Sc, cineretus et $S c$. capistratus dans le nord de l'Amérique. Un autre exemple non moins remarquable est celui d'un Écureuil de l'Amérique méridionale que j’ai décrit, pour cette raison même, sous le nom de $S_{c}$. variabilis. Voyez Notice sur plusicurs espèces nouvelles du genre Ecurenil, dans mes Émdes zoologiques ou dans le Mregrasin de Zoologic; ammée 183 . 
du membre postérieur, et l'autre, médiane. Entre

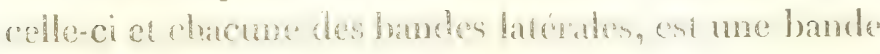
plus large d'un gris clair : c'est dans cette bande grise que sont comprises les mamelles.

Chez un autre individu, également femelle, je trouve une disposition analogue, avec des nuances

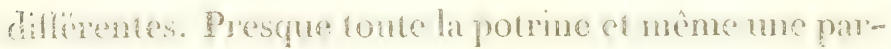
lie du dessous du col, sont d'un roux très-vif qui se prolonge en perdant peu à peu de sa vivacité, en trois bandes, l'une médio-ventrale, les autres latérales. La queue, chez cet individu, est fortement nuancée de roux.

J'ai le reguet de ne pouvoir faire comnaître de quelle localité viennent les deux individus précédents. Celui dont il me reste à parler, et qui est assurément le plus remarguable de tous, vient lu Nexigue. Celui-ci, femelle comme les précédents, a la nuque et la croupe plutôt teintées de roux, que véritablement rousses, et il offre en dessous une disposition très-curieuse. Tontes les parties inférieures sont d'un roux vif, saufhuil taches circulaires grises, ayant un peu moins de 2 centimètres de dianètre, et dont chacune a, non à son centre, mais vers sa partie antérieure, l'un des huit mamelons. Entre la première et la seconde tache de chaque côté, on remarque quelques poils gris qui, à droite suriout, lient manifestement ces taches l'me à l'aure, et tendent à rappeler la disposition en bandes que nous ont présentée les deux individus qui vionnent d'ête décrits. Cette disposition existe même doja assez distnctement en arriere: la moisiome et la 
quatrième tache sont, en ellet, réunies par des poils gris beaucoup plus nombreux, et tellement, qu'à ne considérer que le bas-ventre, il existe entre cet individu et le précédent une ressemblance presque conplète. Au contraire, la seconde et la troisième tacho de chaque coté, sont parfaitement jsolées.

J'ai fait représenter ${ }^{1}$ cet individu fort remarquablet par' la coloration de ses parties inférieures, ainsi que son crâne. 11 importe de noter, à cause l'un caractèru qui sera tont-à-l'heure mentionné à l'égard d'une autre espèce, que la cientition de cette espèce est cell que l'on trouve ordinairement chez les Ecureuils. It existe quatre màchelières de chaqque còté et à chaque mâchoire, et de plus, supérienrement, en avant de lá première mâchelière, une fausse molaire. Celle-ci est d'ailleur's tellement petite et si érroitement serréc contre les premières màchelières, gru'on la prendrait pour un simple tubercule de celle-ci.

2. L'Écureuth de Néboux, Sciurus Nebourï, Is. Geoff.

Je dédie celte espèce à M. Le docteur Néboux, qui I'a découverte, el aux recherches duquel sont dus unssi la plupart des Nammiferes et des Oiseaux de lat Jénus.

C'est au Perpu, at Payta, que M. Naborx s'est pro-

1 Dans la planche XI. - On poura compares cet indiviou à sleux autres varietes do la meme espece (l'une anomale par albinisme partiel), representeis dans la planche $X$. 
cure l'individu, matheurensement unique, dont je vais donner la description.

Sa taille est un peu moindre que celle de l'espèce précédente : elle est de 27 centimètres, du bout du museau à l'origine de la queue, et celle-ci a de même près de 3 décimètres.

La couleur générale du pelage est le gris. Cette coulew, plus ou moins modifie, est en wifit celle de l'animal entier, moins les oreilles, les moustaches et les quate patres qui sont noires, et une tache blanche, comprosece de poils beancoup phus longs que les autres, et situce sur le col, à peu de distance de la base des oreilles.

Le gris est d'une nuance pure et qui rappelle la couleur du Capistrate, sur la plus grande partie du dos et sur la face externe des membres antérieurs; mais il passe au noir tiqueté sur la tête, et au fauve sur la parite posterieure du dos, la crompe el les far ces extérieure el postérieure des membres, ainsi que sur la base de la queue. Dans la portion qui est d'un gris par, les poils sont noit's avec leur extrémite; dans la portion roussatre, ils sont de même noirs, mais avec l'extrémité fauve.

Les parties inférieures du corps et les parties internes des membres, ainsi que le tour de la bouche, sont d'un gris clair légèrement teinté de jaune. Les poils des côtés du ventre ont les deux mêmes couleur's que les poils du dos, mais sont beaucoup plus courts. Les poils plas esuris qui convent le milieu du vente et de la poitrine, présentent aussi les deux mêmes 


\section{$-163-$}

couleurs; mais avec celte diflérence que le blanc ou le fauve occupe la plis gir rela partie de leur cirndue.

La queue, ronde et non distique, est, sauf sa base dont la couleur a été plus haut indiquée, d'un gris blanchàtre, les poils ćtant noirs dans leur première portion, puis blanes vers leur exurémité. De plus, parmi rux, sout entremiles quelques poils enticrement blanes.

Les grandes dents antérieures ${ }^{1}$ sont d'un jaune orangé antérieurement, et blanches dans le reste de leur étendue. Ce sont, comme on le voit, les mêmes couleurs que chez le Sc. aureogaster (et chez la plupart des Ecureuils); mais ce ne sont pas exaciement les mêmes formes. Ces dents, chez le $S$. Nebouxii, sont sensiblement plus petites, plus serrées l'une contre l'autre, notamment à leur sortie des alvéoles, et dirigres parallohemrat, tandis qu'dles sont obliques l'une sur l'autre chez le Sc. aureogaster. Toutes ces dispositions sont en l'apport avec la forme, un peu plus rétrécie, du museau chez le $S$. Nebouxiz.

Enfin, il est entre ces deux espèces, relativement au systime dentaine, une antm: diflience nus-rligue

1 Je me sers ici à dessein de ce terme vague, et qui, tiré seulement de la position des grandes dents des Rongeur's, n'implique aucune détermination. En effet, ce n'est pas le lieu d'examiner si les grandes dents antérieures doivent être considérées, selon leur détermination ordinaire, comme des incisives, ou, selon la détermination de mon père, comme des canines. Voyez le Mémoire que mon père a publié sur cette difficile question de zoologrie, dans les Mémoires de l'Acalćmie des sciences, t. XII. 
d'être notce. Notre individa n'a pas le moindre vesrige de la petite fausse-molaire que j’ai décrite chez le Se. aureogaster, el que l'on trouve presque toujours chez les Ecureuils: la place i laquelle on l'observe ordinairement, est occupée, chez le Sc. Nebouxï, par une racine de la première mâchelière; racine trèsconsidérable, en grande partie apparente au deliors de l'alvéle, et se portant directement en avant.

Au lieu de la formule dentaire que l'on trouve chez le Sc. aureogaster, et yui est aussi celle de presque ious les Scinridés ${ }^{1}$, sarvoir:

$$
\left.\begin{array}{r}
2(M+m+4 M) \\
+2(M \ldots+4 M)
\end{array}\right\}=22
$$

la formule dentaire du Sc. Nebouxil est done, commo on vient de le vois,

In y comprenant méme tres-vraisemblablement quelquesuns des Ecureuils que le doetcur Bachman at décrits comme ayant seulement quatre molaires à chaque mâchoine. (Voyez les Procrerimgs of the zool. socieir, an. 1838, p. 85, et les Annals of natmat history, 1839, 1. 111, p. 275.) D’après l'analogie cxtérieure, j’o-

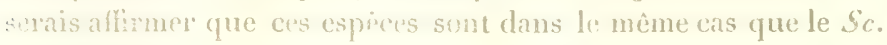
uneosuster, est-à-dire qu: la liusse molaire est excessivement metite, diflicile mène à apercevoir, et d'ailleurs vaisemblablement catibupue.

- Bans cette formule j’ai désigné par $\mathbf{M l}$ les grandes molaires on mâchelieres, par m les petites molaires, et par R les grandes dents intérieures caractéristiques de l'ordre des rongeurs.

Voyez sur ces nouvelles formules dentaires le mémoire que: jai recmment publie dans les 1rchives du Huséun d' Hisioive naaurelle sous le titre de: Deseription des mammifires nouveaux ors innarfuirment connus dela collection du Buscum. 


$$
\begin{gathered}
-104- \\
4(M+4 M)=20^{1}
\end{gathered}
$$

Qant à sa caractéristique, on peut la donner ainsi: Pelage d'un gris tiqueté, passant au fauve postérieurement; les oreilles et les quatre pattes noires. Quene ronde, à poils noirs et blancs, paraissant grisâtre dans son ensemble.

IIl est it peine utile de faire remarquer que la formule

$$
4(\boldsymbol{R}+4 M)=20
$$

"st ici pour

$$
\left.\begin{array}{r}
2(\mathrm{R}+\mathrm{M}) \\
+2(\mathrm{R}+\mathrm{M})
\end{array}\right\}=20
$$

Il y a ici une abréviation, ou pour mieux dire, une simplification résultant de la similitude des dents des deux mâchoires. 



\section{EXPLICATION DES PLANCHES.}

\section{PLANCHE $1^{\text {ro }}$}

Le Cercopithèoue Demalande, Cercopithecus Lalandii, Isid. Glofrorasto-Illitar.

[Au tiers de la grandeur naturelle.]

PLANCHE II.

Crânes et cerveaux de Saimiris, comparés à un crâne de $\mathrm{C}_{\mathrm{AL}}$ Litriche et à un cerveau d'Ouistiti.

[Toutes les figures de cette planche sont de grandeur naturelle.]

Figo. 1. Crâne et mâchoire inférieure du Callitricae a rasọde noin, Callithrix personatus, Geofr. ST.-HiL., vus de profil.

Fig. 2. Denís supérieures du même Singe, vues par la couronue.

Fig. 3. Mâchoire et dents inférieures du même animal, vus par dessus.

Fig. 4. Crâne et mâchoire inférieure du Samiri a dos nRoLE், Saïmiris ustus, Is. Geofr. Sr.-HIL.

Fig. 5. Le même crâne, vu par dessous.

Fig. 6. Le même, vu par dessus.

Fig. 7. Crâne du Sarmer sciurix, Saïmiris sciureus, Is. Grofr. ST.-HIL., coupe verticale.

Figror. 8. Crâne d'un autre individu de la même espèce, vu par la face inférieure. 
ligr. 9. Crâne d'un jeune suję de la même espèce, vu do profil.

ध, r. 3 0. Cerveau d'un autre Samiri sciuris, yu par la base.

Fig. 11. Le mème cerveau, vu par la face supérieure.

Fig. 12. Cerveau d'un Ovistiti ondinanie, Hapalc jacchus, vu par la face supérieure.

\section{PLANCHE III.}

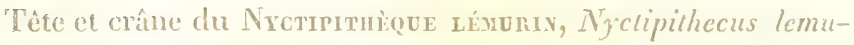
rimus, Is. Groff. St.-HıL., et crâne du N. félix, N. felinus, Six.x.

[Toutes les figures de cette planche sout de grandeur naturelle.]

Fig. 1. Face du NrctiputuèQoe rénonin, chez un individu de couleur ordinaire.

f.... .. Face d'un autre individu de mème espèce, chez lequel to pelage offre une teinte rousse très-prononcie.

H.r. 3. Crâne d'un individu adulte de cette espèce, vu de profil.

Fi... L. Le même crâne, vu par dessous.

ï... 5. Le même, vu par dessus.

Fig. 6. Contours du même crâne, et spécialement des orbites, vus de face.

Fị̛. 7. Crâne d'un jeune individu de la même espèce, vu de. profil.

Ḥ̇. 8. Le même crâne, vu par dessous.

Fig. 9. Le même, vu par dessus.

Fig. 10. Crâne du Nxctipituèǫue félin, adulte, vu de profil.

Figr. 11. Le même crâne, vu en dessous.

Fig. 12. Le même, vu en dessus.

Fig. 13. Contours du même crâne, et spécialement des orbites, vus de face.

\section{PLANCHE IV.}

L'ovas mon, Ursus arctos, Linné, variété du Kamtschatka.

[Au septième de la grandeur naturelle.] 


\section{PI.ANCHE V.}

Squelette de l'Oors ternirue, Ursus ferox, Lewvis et Cuake.

[Au cinquième de la grandcur naturelle.]

Le sternum n'ayant pas été trouvé avec le reste du squelette, n’a pu être représenté.

\section{PLANCHE VI.}

Le: Raton laveur, Procyon totor, S'tonr, variété mexicaine.

[Au tiers de la grandeur naturelle.]

\section{PLANCHE VII.}

La Moupette mésomèze, Mephitis mesomelas, Lichtensten.

[Aux trois-cincuièmes de la grandeur naturelle.]

A. Son crâne, vu en dessus.

B. Le même, vu en dessous.

C. Le même et les dents, vus de profil.

D. La mâchoire inférieure et ses dents, vues par lia couronne.

[Les figures A, B, C, D sont de grandeur naturelle.]

\section{PLANCHE VIII.}

Le: Cinat aibescent, Felis albescens, Pucheran.

[Au tiers de la grandeur naturelle.]

\section{PLANCHE IX.}

\section{Le Cinat an, Felis rufa, Guldenstedt.}

La figure 1 est au tiers de sa grandeur naturelle; les figures 2,5 et 4 sont de grandeur naturelle.]

Fig. 1. Individu, en pelage d'hiver, venant de Californie.

$F_{\text {g g. }}$ 2. Crâne du même individu, vu par dessus.

Fig. 3. Même crâne, vu par dessous.

Fig. 4. Crâne d'un autre individu plus âgé, vu de profil. 


\section{PLANCHE $\mathrm{X}$.}

L'Ecureulu a ventre dorí, Sciurus aurcogaster, Fr. Cuvien; variétés.

[Aux deux tiers de la grandeur naturelle.]

L'un des deux individus figurés dans cette planche est une variété par albinisme partiel.

L'autre individu offre un exemple des variations normales du pelage dans cette espèce. Le ventre est gris, et non roux, comme dans les individus jusqu'à présent décrits ou figurés.

\section{PLANCHE XI}

L'Ecureull a ventre doné, Sciurus aurcogaster, Fr. Cuvier; variétés.

[ Figure 1 aux deux tiers de la grandeur naturelle; figures 2 et 5 de grandeu: naturelle; tigure 4 grossie.]

Fig. 1. Individu femelle, à ventre roux, avec le tour des titines d'un gris blanchâtre. Il offre ainsi réunies daus la région abdominale la coulcur grise observée chez l'individu précédent, et la couleur rousse, regardée par les auteurs comme caractéristique de l'espèce.

Fig. 2. Le crâne, vu en dessus.

Fig. 3. Le crâne, vu de côté.

Fig. 4. Les dents de la mâchoire supérieure.

\section{PLANCHE XII.}

L'Ecoreurl de Néboux, Sciurus Ncbouxii, Is. Geoff. St.-HiL.

[ $L$ 'individu entier, aux deux tiers de la grandeur aturelle; les figures $\Lambda, b, c$ de grandeur naturelle; $\mathbf{D}$ grossie. ]

A. Son crâne, vu par dessus.

B et C. Le même et les dents, vus de profil.

D. Les dents, vues par la couronne. 


\section{PLANCHE XIII.}

Le Níotome de ra Floride, Neoloma Floridana, Ord et Say.

[ L'individu entier de grandeur naturelle; les figures $\Lambda, b, c$ de grandeur naturelle, $\mathbf{B}$ et $\mathbf{C}$ grossies.]

A. Mâchoires etdents, vues de profil, de grandeur naturelle.

B,b. Dents de la mâchoire supérieure.

C, c. Dents de la mâchoire inférieure.

Les unes et les autres vues par la couronne. 



\section{INDEX}

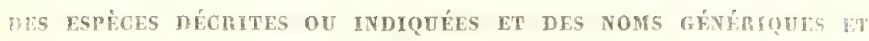
SPÉCILIQUES CITÉS DANS CE TRAVAL.

Aote, Aotus (syn. de Nyctipithéoue, Nrc-| C. tantale, C. tantalus. . . . . . . TIPITHECUS). ......48-49, 113 Aotes (pour Aotus)........ 115

Cillitriche, Cillitirix. 105-107, 111 -

C. Temminck, C. Temminckii. . . . $2 / 4$

C. vervet, C. pygerythrus. . . 8-11, 29

Chat, Felis. . . . . . 157-15\%

C. albescent, F. albescens. . 1, 1, 14 !

115 C. bai , F. rufa. ..... 140-1,iz

Callithrix d'Erxleben.. . . . 55-45 Colobe, Colobus.. . . . . . . . 6

Gebus d'Erxleben........ 55, 41, 42 C. ferruginosus... ........ 90

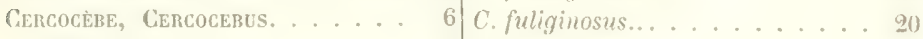

C. athiops........... 19 C. Gueresa........ . . . . . . . . . . . . .

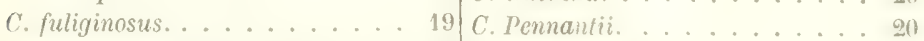

Cencopitheque, Cercopithecus.. 6, 19-54 C. polycomos. . . . . . . . . .

C. blane-nez, C. pelaurista. . . . . g- C. Satanas.. . . . . . . . . . . 20

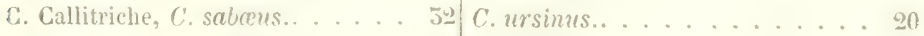

C. Camplell, C. Camplelli. .... o. C. vellerosus.. . . . . . . . . 20

C. Delalande, C. Lalandii.. .11-18, 28: C. verns. . . . . . . . . . .

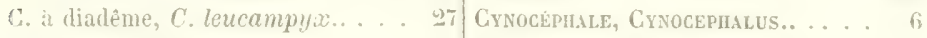

¿. Diane, C. Diana... . . . . g

C. a dos rouge, C.pyrrhonotus. . . 5i

C. Grivet, C. giseo-viridis......51/Ecmreuil de Nëboux, Sciurus Ne-

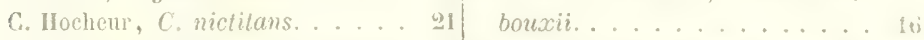

C. aux lèves blanches, C. labiatus.. 25 E. a ventre doré, S. aureogaster. . I:

C. à longue barbe, C. pogonias.. . . 22

C. Malbrouck, C. cynosurus.. . . . 30

C. Martin, C. Martini... . . . . 24

C. Mone, C. Hona... . . . . . . 2

C. Monoïde, C. monoüdes.. . . . . .

C. Moustac, C. cephus.. . . . . .

C. à oreilles rouges, C. erythrotis............ . . . 25

C. Patas, C. ruber....... 55

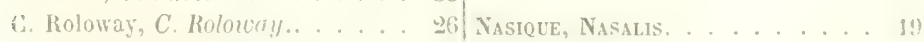

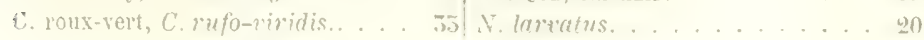


NEOTOME dE LA Floride, Neotomo floridana...............

Nocthore, Nocthora (syn. de NictiPITHEQUE, NyctipitHECUS).. . 49, 115

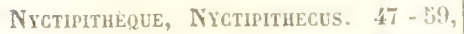

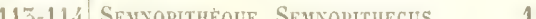

N. criard, $N$. vociferans.. . 64,117 S. albo-cinereus (espèce nominale).. 20

N. félin, N. felinus. ... $65,67,11$; s. auratus........... 19

N. lémurin, N. lemurinus. . . 70, 115 × ris/n/us. . . . . . . . 19

N. Miriquouina. ...... 59, 117 s. cucnllatus. ......... 19

N. à trois bandes, $N$. trivirgatus. 61, S. Dussumieri.......... 19

65,116 S. entellus.............. 19

S. femoralis......... 19

Ouns brun, Ursus arctos, var. . . 121 S. latibarbatus.......... 19

0. terrible, U. ferox....... 125 s. fla immms. . . . . . . 1!

S. fulvo-griseus (espèce nominale).. 20

Pithesciureus (syn. de Saimis). . . 47 S. leucoprymmus. . . . . . . 19

S. matrus. . . . . . . 19

Raton, Procyon...... 125, 152 S melalophos. ......... 19

R. laveur, P. lotor, var. ..... 12S S. mitratus........ 19

S. nemreus........... 19

S. nigrimanus.............. 19

S. olscurus........... 19

S. rubicundus......... 20

divers).........57, 47, 111

Saïmin, Saïmis. . . . 450-47, 77-92, 107,109

S. à dos brûlé, S. ustus. 95, 96-99, 110 ThÉropithènue, Theroprthecus.. . . 6

S. entomophage, S. entomophagus.. 95, Troglodyte, Troglodytes. . . . . . (; 99,111 


\section{OISEAU X,}

Par MiM. Fu. PRÉtost et O. DES MURS.

Les Oiseaux de l'expédition de la Vémus composent la plus riche partie des ricoltes scientifiques faites par les (Miciers naturalistes de cette frégate. C'est pour les Vertébrés ce qu'ils ont rapporté en plus grand nombre; c'est aussi ce dont ils ont rapporté le plus de nouveautés. Douze espèces composent, en effet, leur part de découvertes en Ornitholosie. Or, si l'on se rend compte des dilticultés qui viennent accabler les officiers d'un navire, lorsqu'à l'accomplissement de leur's devoirs et de leurs obligations du bord, ils veulent joindre le tribut de leur zèle pour la science, on comprendra qu'ils n'arrivent que péniblement à réunir en quantite sulisante les matériaux nécessaires aux progrès d'mne science aussi multiple que l'histoire naturelle, et l'on doit leur savoir d'autant plus de gré des efforts qu'ils font à cet égard, que c'est pour cux un surcroît purement gratuit de peines et de fatignes dont ils ne trouvent le faible 
prix que dams la publication des résultats de leurs tratvaux. Aussi nous estimons-nous heureux d'avoir été

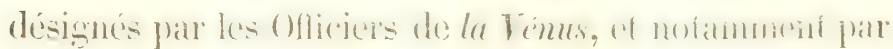
M. le docteur Néboux, son chirurgien-major, pour être

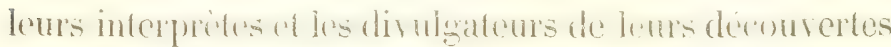
et de leurs observations Ornithologiques.

Si importantes que paraissent cependant ces découvertes et ces observations, on conçoit qu'elles ne sont pas assez complètes pour nous permettre une oeuvre d'ensemble, ou des déreloppements aussi étendus que nous l'eussions désiré. Il faut à de semblables travaux des séries d'individus d'un même genre, souvent même de la même espèce, et c'est ici ce qui nous manque, par le fait même des circonstances au milieu desquclles s'est eflectuée l'expédition, de la brièvelé de ses stations et de la rapidité de son parcours. Une circonstance entre autres est venue diminuer une partie de l'intérêt qui devait s'attacher aux résultats de cette expédition. La portion la plus riche de ses découvertes et de ses récoltes scientifiçues est celle relative à la Haute-Californie et aux Gallapagos: or, le malheur a voulu que la Vénus commençàt à parcourir ces parages si curieux et si riches au point de vue Ornithologique, au moment même où le navire anglais le Beagle venait de les explorer et les quittait. Il s'en est suivi que notre

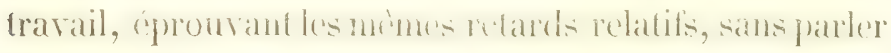
de ceux dus aux événements politiques survenus en France, n'a pu venir qu'en second ordre; c'est ce qui nous a rendus si sobres dans la représentation des

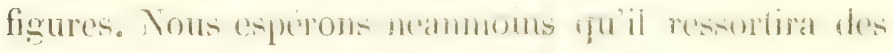


notes de MIM. Néboux et Fillieu (ce dernier commis de la Larine à bord de la Vénus) jointes à nos descriptions, un intérèt qui justilie suflisamment l'empressement du Ministère de la Marine à en faciliter la publication. Cet empressement, du reste, qui n'est que l'expression de l'hommage que rend le Gouveruement à la science, et l'accomplissement d'un devoir bien entendu de sa part envers ceux qui la cultivent, a déjà trouvé sa justification dans le concours si dévoué aux encouragements et aux progrès seientifiques donné par un des nembres les plus distingués de l'Institut, M. Isidore Geoffroy Saint-Hilaire à M. le contre-amiral Dupetit-Thouars, chef de l'expédition de la témus, pour la mise en ouvre des matériaux relatifs à la partio Mammalogique de ce voyage.

Sans aroir la prétention d'atteindre l'élévation de rues el d'appréciations du Savant Académicien, nous suivrons, à peu de chose pris, la ligne tracée par lui pour ces sortes de publications dians de précédents travaux : e'est notre devoir comme disciples de l'illustre Professeur; c'est de plus la condition indispensable à l'unité d'ensemble et à l'harmonie de ce livre en tant qu'ourrage d'histoire naturelle: c'est du reste ce que nous arons déja fait dans d'autres publications ${ }^{1}$.

Nous ne nous bornerons donc pas à la simple des-

1 Voyage en Abysinie, du lieutenant de marine Th. Lefebvre (partie Zoologique).- Histoire politique et naturelle du Chili; par M. CI. Gay (partie Ornithologique). 
cription des esperes figurées dans len dix planches qui composent l'atlas Ornithologique de la Vémus. Nous y joindrons, en dehors de ces descriptions des densiderations auxquelles elles pourront donner lieu de notre part, la reproduclion de quelques détails sur d'autres espèces déjà publićes soit par M. le docteur Néboux, soit par M. Léclancher, son aide, toutes provenant de la mème expédition. C'est, nous le pensons, le seul moyen de rendre plus utile en le complétant, un travail tel que le nôtre, purement scientifique; nous nous faisons même un devoir d'ajouter que ces excursions que nous croirons devoir faire en dchors des notes spéciales à nos 10 planches d'Oiseaux, nous ne nous les permettrons que d'accord avec M. Néboux, et de son autorisation expresse: ce zélé Naturaliste nous ayant témoigné l'intention de faire taire toute autre considération de prérogative, de droits ou d'amour-propre, en présence d'un service à rendre à la science.

Enfin, nous compléterons autant que possible notre travail par l'indication de quelques espèces bien connues, mais dont l'habitat a été constaté par le docteur Neboux en dehors de la circonscription geographique qui leur étail jusquàa présent assignée, surtout en latitude septentrionale. 


\section{CARACARA VULGAIRE.}

Polyborus Brasiliensis, Swains.

vulgaris, Vieill., Gal.des Ois., pl. 7; Spix, Av. Bras., tom. $1^{\circ}$, tab. $1^{a}$.

Falco cheriway, Jacr.

Vultur plancus, Lath.

Falco Brasiliensis, Gm.

- Tharus, Mol.

Jusqu'à ce jour, ce Falconidé ne s'est encore communément trouvé que dans l'Amérique méridionale, à laquelle on le considère comme exclusivement propre : ce ce qui n'a pas empêché qu'on ne l'y ait rencontré sur les deux côtes de ce continent, à l'est, repuis le Brésil, à l'ouest, depuis le Chili jusqu'à la pointe la plus australe, la Terre-de-Feu et le Cap Horn; en remontant vers le nord, MI. Gould avec les voyageurs naturalistes du Beugle ne les fait pas remonter au delit de la pointe extrème de la Floride, c'est-à-dire, à environ 20 degrés de latitude septentrionale, car c'est à peine si à celte latilude on avait constaté sa présence dans l'Amcrique septentrionale.

M. le docteur Néboux l'a rencontré à Nonterey, (Haute-Californie), d'où il en a rapporté plusieurs exemplaires; en sorte que l'habitat de cel oiseau se trouve reculé par le fait au nord, de près de 10 degrés. 


\section{PROCNE MODESTE.}

Procne modesta (Gould.), Zool. Beagl., p. 39, pl. 5. Hirundo concolor (Gould.), Proc. zool. Soc., 1859. modesta (Néboux), Rev.zool., oct. 1840, p. 291.

Char. spec. Pr. (Mas.) nitidè corulescenti-nigra (Gould). Loc. cit.

Le docteur Néboux a rapporte de cette espèce la femelle dont la couleur générale est d'un gris-brun; elle est plus petite que le mîle; longueur totale, 14 centimètres.

Habit. L'île Saint-Charles des Gallapagos. 


\section{NOTICE}

SUR LE

CENRE IIETIG TATILE (II'mignahus, Lichtenstein), $(183 \%)$.

Heterorhynchus ( La Fresnayc), 1859.

La perfection de toutes méthodes naturelles, c'est de se montrer rationnelles et logiques. Ce qui s'oppose en général, et ce qui s'opposera toujour's à cette perfection, est l'impossibilité de fixer une limite aux deux extrêmes de chaque division qu'on y introdnit; impossibilite telle qua de logiques of rationmelles quidles devraient paraitre, elles semblent d'ordinaire, sinon suivre une marche contraire au raisonnement, du moins arriver à un resultat presifue diamétralement op)posé à celui qu’elles laissent entrevoir.

Ainsi, parfois on y remarque, mais bien rarement, un ensemble des phus satisfatiants de caracteres organiques cher les individus composant une, série Zoologique: plus sonrent, au lieu de eet cusemble, on y voit des rapprochements en quelene sorte monstrueus (quant à l'apparence de ces caractères. Mais alors les vides et les. intervalles si brusfuement franchis se trouvent comblés par des caracteres tirés d'un autre ordre d'idées où ces rapprochements d'aspect si hétérogene trouvent leur 
laison d'êtro; c'est-à-dire que, forcé que l'on est de concilier le classement par indication d'organes avec le classement par indication de moeurs ou d'habitudes, on en est réduit à emprunter à l'un ce qui manque à l'autre, et réciproquement; d'où il suit que les méthodes, en voulant tout à la fois parler aux sens, c'est-à-dire à la vue et à l'esprit, ou mieux encore, à la raison, ne satisfont jamais complétement celle-là qu’aux dépens de colle-ci; de lì, lour mobilité; de lì cigralenent leur défaut d'unité de diagrnoses génériques, ot leur peu d'applicabilité spéciale aux qunres qu'elles veulent caractériser; la plupart de ces caractères pouvant plus ou moins s'appliquer à une infinité d'autres genres. En sorte qu'en definitive les méthodes jusqu’à présent ont. plus aidé, dans une certaine mesure, à la mémoire de ceux qui s'oceupent d'histoire naturelle, gu'olles n'ont simplific ou fail progresser la science. Peut-chre, après lout, la faute en est-elle à l'absence; chez les méthodistes, de toule idée philosophique, sans laquelle il ne peut jamais y avoir de classification passable. Nous nous empressons toutefois ici de faire exception, nous ne dirons pas au blâme, car ce n'est qu'une opinion persommelle, mais au regret que nous exprimons à ce sujct, en fareur d'me nourelle méthode introduite dans la sience par notre savant maitre ot ami II. Isidore Geoffroy Saint-llilaire. Nous roulons parler du parallélisme appliqué à la chassification naturelle, méthode inspirce par une idée philosophique dont le développement el l'application permettent d'espérer les progrés les plus sérieux. 
Ces réflexions nous sont suggérées par l'Hémignathe que nous figurons. Cet oiseau est sans contredit le plus curieux de l'expédition de la Vénus; par l'ensemble de ses formes et de son port, il est bien évident qu'il rappelle au premier aspect les formes et le port des Souimangas (Necturinia) : aussi le docieur Néboux l'indiyuat-il pour tel dans les notes de son voyage, comme dans la note insérée par lui (Revue zoologique, 181.0, page 289).

En effet, à part la forme si anormale de sa mandibule inféricure, le developpenent de la mandibule supérieure et l'are de cercle décrit n'offrent rien de beaucoup plus extraordinaire que chez d'autres esperes de Souïmangas, notamment le Cymniris (Terentinia) anea de Vieillot, liguré par Levaillant, ()is. d'Ifr. pl. egr : car la corde de cet are de cercle n'excèdle celle du Cinmyris anea que d'un 1/2 centimètre, notre oiseau mesurant 3 centimetres, ol ce dernier 2 centimetres $1 / 2$, en mesurant la corde depuis les narines jusqu'à la pointe, et le Drepranis coccinea inférieur à celui-ci de 3 millimètres.

Pourtant en examinant la forme de la mandibule inferricure si peu proportionnce à la supérieuro, la forme des tarses et le développement des pennes, on ne peut. s'empecher d'y saisir des diflérences comparatives no. tables.

C'est cet examen qui porta M. de La Fresnaye, dans le Magasin de zoologie, 1839, à faire de cette sorte d'Oiseaux un sous-genre auquel il domna le nom d' $H e$ terorhynchus, oubliant que le genre II mignuthus avai 


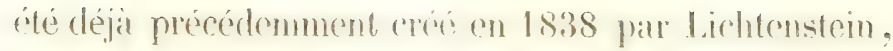
et que son espèce, qui est aussi la nôtre, avait été décrite par ce naturaliste qui en avait fait le type du genre.

Voici, à ce sujet, comment s'exprimait l'honorable M. de La Fresnaye :

« La forme toute anormale du bec rle cet Oiseau ) semble autoriser, au premier abord, la formation ) d'un genre nouveau; mais en comparant ses pattes, ) ses ailes, sa queue et même la grande courbure de ) son bec avec ces mèmes parties chez l'Héorotaire ves) tiaire, habitant les îles Sandwich comme Iui, on est ) frappé de leur grande analogie, et la diflérence seule " du bee, quoique assez marquée, ne nous a paru in" diquer qu'une distinction sous-gónérique entre eux. » Puis il en établissait les caractères en ces termes:

“ Bec allongé, très-arqué, formant exactement un s "fuart de rerole; mandibule superienre un peu chargie ) à sa base, et retombant sur l'inférieure, puis rétrécie "subitement et très-comprimée, creuséc intérieure") ment jusqu'à moitić seulement de sa longueur, et de " là jusqu'à la pointe, cylindrique, très-mince et très"pointue; la mandibule inférieure beaucoup plus ) courte et plus épaisse qu'elle, et n'atteignant que la ) moitić de sa longueur, creusée dans toute sa lon"gueur, comme de coutume, et recourbéc comme la ) supérieure; pieds robustes, tarses, doigts et ongles ) allongés, le pouce et son ongle très-forts; ailes cour»tes, à rémiges subobtuses; queue terminée carré) ment. " 
M. de La Fresnaye était donc dans le vrai en cherchant a séparer son nouvel oisean de l'Iféorotaire cicarlate, Mellitherpus vestienius , Vieillot), Certhie coccinea (Gmel.), et en faisant un sous-genre. Suivant nous, néanmoins il n'osait pas assez, car ses caractères sont assez tranchés et assez distincts de ceux de ce dernier pour en faire la base, non pas d'un sous-genre, mais d'un véritable genre.

C'est effectivement de la sorte que l'avait envisagé Lirhlenstein, lorsqu'il elevar nouvel oiseau au rang de senre sans cepentant en assigner les caractions autrement que par la composition d'un nom générique qui en indigne an moins le principal. Ifin de completer l'ensemble de ces caractires, nous allons les rendre ici tels que nous les saisissons :

Le bec est excessivement allongé et arqué, non dans le sens du prolongement de la ligne frontale, mais en se relevant brusquement à partir de sa base, le sommet de lare de cercle dépassme ainsi le niveau du front: la mandibules inferieure alteint a peine la moitić de la mandibule supérieure qui est d'abord dilatée dans son premier (fuart, ensuite comprimée of se termine en aline arronrlie et fine comme une aiguille: les pierls sont forts; l'ongle du pouce est plus développé que les autres; la quene est très-courte; la base du bee st garnie de quelques poils noirs.

Pour se rendre eompte des différences organiques qui apparaissont cntre l'Hemiquntus lurilus ef Certhie (Drepanis) vestiaria ou coccinea, il suffit de les comparer. 
f.e Vestiaria ofire pour caracteres les suivants: Bec plus longy que la tète, épais, très-recourbé, pointu, à arcte arrondie, à bords lisses; la corde de l'arc de cercle dessiné par sa courbure mesurant 22 millimètres; les deux mandibules paralieles et s'accompagnant jusqu'a la pointe, par conséçuent d'égale longueur; narines ovalaires, basales, recouvertes par une pellicule; ailes peu obtuses: la deuxieme et la troisième rémiges d'égale longueur; la promiere la plus courte. Tarses, minces, grềes, scutellés, torninés par trois doigrts faibles, l'externe soudé à sa base dans presque toute la longueur de la premiere phalange, pouce avec son ongle ciépassant à peine la suoitié de la longueur du tarse.

Maintenant, nous le demandons: quel ensemble de rapports peut-il oxister entre un Oiseau à mandibules rigales, à bec dont la courbure offre une corde de 22. millimètres de longueur, à tarses minces et grêles, ì ongles faibles, à pouce égal à peine à la moitié de la longueur du tarse;

Lit un oiscau à mandibules inécgales, l'inférieure n'attcignant qu'à moitié de la supérieure, celle-ci formant un arc de cercle de 30 millimètres; à pieds et ongles robustes, à pouce égal à la longueur du tarse?

Avec une distance telle et une pareille diflérence entre ces deux termes, nous comprenons bien le rapprochement, par transition, d'un genre à un autre, mais nous ne comprenous pas le rapprochement d'espèce à espèce, ou pour mieux dire la confusion des deux espèces dans un mème genre.

C'est pourtant ce qu'a fait M. G. R. Gray, qui, dans 
Gener. of Birds, juin 184.7, a compris ces deux especes si exclusives l'me de l'autre, dans son genre Drepanis; et en cela nous estimons qu'il n'a été ni dans la vérité ni dans la logique de la méthode naturelle.

Non pas que nous prétendions avoir quoi que ce soil. à redire à la composition du genre Drepanis, en tant. que coupe géographique; mais comme division grenérique nous ne nous expliquons point pourquoi par exemple le Cimmpris renea n'y liguremail pas aussi bien que Certhia vestiaria, et réciprofuement pourquoi ces dernier s'éloignerait du genre Necturinia plutot que le premier dont le bee est méme encore abs mu plus longr et tout aussi courbé.

Quoi qu'il en soit, ce qui résulte de ces réflexions, c'est que cest ì bon droit que Lichtenstein a ronstitue son genre Hemignathus. Il est mome remarquable que le type de ce genre soit l'espèce publiée el figurée par $\mathbf{M}$. de La Fresnatye sous le nom d'Iteterorhynchus olivaceus, et par nous sous celui d'Themignathus, d'apres un individu de l'expédition de la lémus, mais ì laquelle son fondateur avait donné déja le nom de Lucidus, par opposition sans donte à l'Hemignathus obscurus (Certhia obscura de Latham).

Il est bien vrai que M. de La Fresnaye avait élé prévenu par Natterer "que cet Giseau, ainsi qu'il le dit lui"méme, avait été dijat décrit en Allemagne en en Russic ) sous un nom générique nouveau, et qu’il en existail ") même deux espèces différentes, mais l'une el l'autre ») à couleur olivâtre et jaunâtre comme celle rapportée " par la Vémus." 
Mais, induit en erreur par une note de N. Léclancher, il crut devoir révoquer en doute ce témoignage. «MI. Léclancher, à propos de cet Oiseau, me mande, ") continue M. de La Fresnaye : L'H éorotaire à mandibu") les inégales esi plus commun que le Vestiaire, car les "enfunts en apportuient un phas arrand nombre prour vendre; "je n'en ai pas vu de rouges comme le Vestiaire, j'en ai ") seulement écorché qui avaient quelques plumes rouges "sur le dos, ce qui me faisuit croire qu'ils devenaient rouges "avec l'âge. L'espèce toute rouge, le Vestiaire, que j'ai 1) vue au pic I'arry, s'accrochaii aux branches en sautant "plutôt comme les Mésunges que comme notre petil Grim"pereau, et ne se collait point sur leur surfuce comme les "Pics el les Grimpereaux. )

Or, il nous est démontré que l'observation de M. Lé-

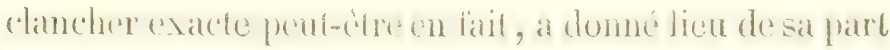
à une induction complétement erronée, car aucun des individus rapportés par le docteur Néboux, et aucun de ceux qu'il a eu occasion d'observer sur les lieux n'a présenté le moindre vestige de rouge ou de rougeâtre; et nous craignons que M. Léclancher n'ait confondu dans sa remarque ou dans ses souvenirs le jeune Vestiaria avec l'Itemi. Lucidus, le premier, à cette

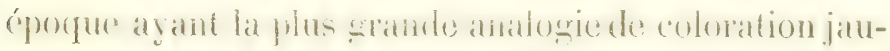
nâtre el olivâtre avec ce dernier: Ainsi les jemes mâles du Festuriu, arant de prendre to plumases briblant de l'adulte, sont d'un janme olivilie aree des points on des

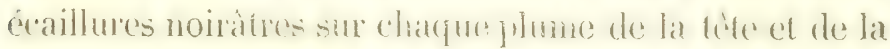
gorge; ef dans leur phunage de transition le fond jaunatre ast parfois strie de: finces plunes romeres d'antant 
plus remarquables dans leur éclat qu'elles sont plus ('lairsemces.

Nons pouvons done affirmer au moins quant à notre espèce, qui est la même que celle observée par M. Léclancher, qu'elle ne devient jamais rouge dans l'état adulte; et nous n'hésitons pas à croire que si l'honorable M. de La Fresnaye eût été instruit de la connaissance qu'avait eue avant lui Lichtenstein, de ce genre, puisqu'il en est le fontateur, il cult dome pleine réance à l'avis officieux de Natterer qui, comme on le sait, ne

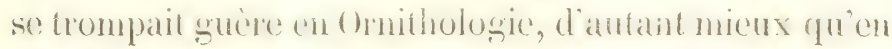
eflet Lichtenstein en fondant son gente', te compusait de deux espèces, à savoir: $1^{\circ}$ II. obscurus (Certhia obscura de Lath.), (qui sans olirir la mène disproportion dans la longueur des denx mandibutes, a coprendint celle inferieure notablenent plus conte que la supkrieure; $2_{i}^{\circ} \boldsymbol{I I}$. lucidus màle, adulte et jeune.

Ce genre se compose done encore aujourd'hui, comme à l'époque à laquelle le créa Lichtenstein, en 1837, de deux espèces:

10 Memignathes obscurns (1,icht.), Mhlanoll. akad. der Wiss., zu Berlin, 1839 , p. 449, tab. 5, fig. 1;

Certhia obscura, (Lath.).

Drepanis - (G. B. Gray.), Gen. of B., $184 \%$.

20 Hemignathus lucidus (Lichtenstein, 1837);

Ineterorhynchus olivareus (de) Lal Frenaye), Hary. de zool., 1839, Ois., pl. 10;

${ }^{1}$ Abhandl. akad. der Wiss. zu Berlin, 1859 , p. 45x, tab. 5, fig. 1, 2, 5 (mémoire lu en juin 1857 ). 
Vestiaria heterorhynchus (Lesson);

Drepanis lucida (G. R. Gray.), Gen. of B., 1847;

Memignuhus olivaceus (Flor. Prév.), Voy. de la Vémus, Ois., pl. 1 ;

Drepanis olivacea (G. R. Gray), Gen. of B., 1847;

Carr rest par crreur du graveur que le nom spécifique d'olivaceus a été mis au bas de notre planche $1^{\text {re de }}$ l'Hemigmulms, arec l'indication de Lichtenstein pour auteur, notre inclication ne se rapportant gu'à la dénomination générique.

Cette réflexion est d'autant plus importante à faire ici que l'erreur dont il s'agil a déjà trompé M. G. R. Gray qui, dans son Generef of B., a reproduit la désignation lautive de notre planche en eonservant sous le nom Drepanis olivacea, une espèce dont ne s'est jamais occupé Lichtenstein, par cette seule raison qu'elle n'existe pas, et ne repose que sur cette crreur chalcographique.

\section{PLANCHE I. - Fig. 1 ET 2.}

\section{HÉMIGNATIEE BRILLANT.}

Hemignathus lucidus (Lichtenstein), Abhandl. akad. der Wiss. zu Berlin, 1859 , p. 451 , tab. 5, fig. 2, 5 , lu en juin 1857 .

Heterorhynchus oïvaceus (de Ia Fresnaye), Mag. de $\approx 001 ., 1859$, Ois., pl. 10.

Vestiaria heterorhynchus (Lesson). Drepanis lucida (G. B. Gray), Gen. of B., 1847.

Hemignathus olivaceus (Flor. Prévost), Voy. de la Jémus, pl. 1, Ois.

Drepanis olivacea (G. R. Gray), Gen. of B., 184.

SPEC, chan. Hem. suprà olivaceus, rapite diluties: superciliis 
t corpore infero lucidé flavis, pectore ferè junquillaceo : striga olivacea à commissurd usque ad aures transeunte

Notre but, en publiant cette figure, avait été, non pas de la donner comme nouvelle, mais de la donner plus parfaite que celle do Lichtenstein, et de grandeur naturelle.

Description. Tout le dessus du corps d'un olivâtre foncé, plus clair sur le sommet de la tête; le front, les sourcils, à partir des narines, les joues, la gorge, le devant du cou et la poitrine sont d'un jaune vif, presque jonquille sur ces deux dernières parties; une large bande d'un olivâtre tournant au noir vers l'oreille, part de la commissure du bec, recouvre le lorum, et va se terminer au méat auditif, laisant ressortir plus vivement le jaune de la bande sourciliere; les petites couvertures alaires sont d'un olive verdâtre; les rémiges secondaires bordées de la même teinte; les rémiges primaires et les grandes convertures bordées de jaunâtre. Bec et pieds couleur de corne bleuâtre. Màle adulte.

Ia femelle est d'un olivâtre obscur et presque fuligineux en dessus, et en dessous d'un brun clair presque couleur de biche; elle n'a de jaune qu'aux sourcils, aux joues et à la gorge; toutes les plumes des ailes et de la queue sont bordées de verdàtre.

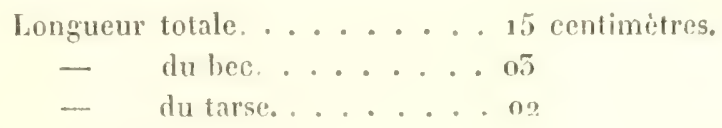

IIabit. Iles Sandwich.

OISEATIX. 


\section{PLANCHE II.-FIG. 1 ET 2.}

\section{OISEAU IIOUCHE DE COSTA.}

Ornysmia Coste (J. Bourcier), Rev. zool., 1859, octobre, p. 294; Ann. des sc. phys., ctc., de Lyon, 1840, p. 225.

Mellisuga Costa (G. B. Gray), Gen. of B., $18 \nmid 8$.

CHar. SPEC. O, mucha et corpore superiore lateribusque metallicè viridissimis; vertice fronte genisque ac gulà, plumulis squamosis indutis purpurescenti-caruleis; illis ex latere colli prolongatis et lanceolatis, cx eneo, granatinoque splendentibus; maculà post-ocularinigro-ccrulescente lined albâ trans-notatd; alterd macula mystacaliformi ex commissuris ad aures descendente, concolore; gutture, pectore, crissoque albis; abdomine albo-cinerascente viridi-nigro squamulato; tectricibus cauda inferioribus albis viridi exlenso squamulatis; rectricibus externis tribus albo apice fasciatis.

La description de cet Oiseau-Mouche, faite dans la Revue zoologique de 1839 (octobre), page 29\%, par M. J. Bourcier, qui l'a nommé et dédié à M. Costa, directeur du Musée de Naples, l'a été sur un jeune mâle

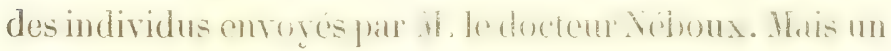
envoi postérieur en renfermait un individu beancoup

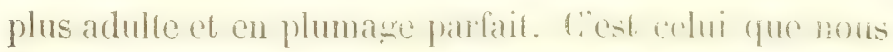

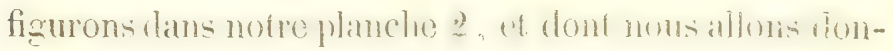
ner une description qui viendra compléter la première.

Description. Mâle adulte. Bec allongé, mince, droit; tout le dessus du corps vert brillant à reflets métalliques; couvertures supérieures alaires et caudales de mème couleur, celles-ci de forme élargie et allongée; dessus de la tête, joues et devant du cou recouverts de plumes 
squammeuses d'un reflet blen-d'acier brun. Mais ces plumes, tout en conservant à la base leur forme d'écailles sur les deux dernicres de ces parties, se prolongent des deux côtés du cou en façon de jabot ou de cravate de la forme la plus sracieuse; clles atteignent alors une dimension qui, pour les plus longues, va jusqu'à 1 centimètre; leur ton général d'acier bruni, en cef élat, emprunte a la lumiere une couleur de grenat clair ou rosé sur les harhules de l'un de leurs còtés, et une rouleur de noir-bleuitre velonté sur les autres barbules; la tige de ces plumes se dessine en blanc formant entre ces deux teintes une strie très-apparente. Une tache post-oculaire d'un noir blenatre qui s'élève un peu sur la partic externe du soureil se troure partagce en denx par une étroite ligne blanche prartant de l'angle externe de l'wil et s'ctendint jusqu'au méat auditif; une autre tache, en forme de inoustache du mime ton noirbleuatre, fart de la commissure du hee ef encadre toute la prartie inférieure de la joue. Tistomac el poitrine, ainsi que la région anale, blanc pur, ventre blanc-grisâtre, moyennement écalles de rert sombre; flancs du mème vert brillant que le dessus du corps; couvertures caudales inferienres blanches lavenent écaillées de rert, bec et pattes noirs.

Les ailes, plus longnes que la quine ef falciformes, sont d'un ton noiràtre; queue triangulaire, avec les deux premières rectries tris-étroites, arrondios et recourbes intérieurement vers l'extrémité qui est, ainsi que celle de la troisieme rémige, bordés d'une tache blanche a la pointe. 
Longuem totale. ........ 75 millimetres.

- du bec......... 19

- de la queue...... ${ }_{2} 6$

Ailes dépassant la queue de. ....99

Jcune mâle. Nous reproduirons pour cet âge la description donnée par M. J. Bourcier : “ Dessus du corps ") a légers reflets vert brillant, poitrine blanche, flancs n et abdomen garnis de quelques plumes aussi d'un vert ") brillant, convertures de la queue larges et longues, ") d'un vert métallique; calotte, joues et devant du cou "recouverts de plumes cicailleuses d'un reflet rle blen ") d'acier bruni. "

Femelle. La femelle manque de tous les ornements du mâle. Elle a le dessus de la tête et le derrière du cou (l'un gris bronitre avee quelques plumes éparses d'un vert plus ou moins doré ou olivâtre; le dessus du corps du mème vert que le màle; les couvertures supérieures alaires d'un vert à reflets dorés; les trois rectrices externes bordées de blane à leur extrémité; le menton et la jone d'un blanc ocracé; une tache grisâtre postoculaire; la poitrine grisâtre, et l'abdomen blanchâtre.

M. le docteur Néboux a trouvé cette espèce avec ses diflérents âges dans les environs de Aunterey, Nouvelle on haute Californie.

Cet Oiseau-Mouche, que M. Bourcier fait entrer dans les Lucifers de Lesson, a les plus grands rapports d'ornementation avec les Ornata, Strumaria, Petasophora, Vieillotii, etc. C'est un des phus remarcguables de l'Expédition. 
TIICHAS VOILÉ

Trichas velatus, G.-B. Gray, Gen., 1848 .

Sylvia velata, Vieill., Ois. Am. sep., rol. 2, p. 74 , d'Orb. et Lafr., Mlag. de zool., 1856, p. 20.

- canicapilla, Pr. Max.

T'anagra - Swains., Ill. orn., pl.174.

Trichas - id.

Trouse a Maldonado par les naturalistes du beagle, à Monterey, Haute-Californic, par le docteur Néboux.

\section{MNIOTILTE A SOURCILS.}

Mniotilla superciliosa, J.-B. Gray, Gen.

Sylvia - Bodd., Pl. enl., 686, fig. 1.

Motacilla pensilis, Gm. Vieill., Ois. Am. sept., pl. 72.

- Ravicollis, Gm.

Sylvia pensilis, Ch. Bonap.

Trouvé à Monterey.

MNIOTILTE D'ÉTÉ.

Mniotilta cestiva, J.-B. Gray.

Molacilla Canadensis, Bodd.

Sylvia citrinella, Will., Am. orn., pl. 15, fig. 6; Vieill., Ois. Am. sept., pl. $9^{5}$.

- astiva, Gm., Gab. Buff., pl. enl. 58, fig. 1-2.

Sylvicola astiva, Swains.

Motacilla albicollis, Gm., Briss. orn., t. 5, tab. 26, fig 5.

Sylvia Childreni, Audub., pl. 55.

Rhimanphus citrinus, Rafin.

De passage dans tout le Continent Américain, émigre en hiver vers les Tropiques, de la Pensylvanie, ou elle est 
commune, d'apris les olservations de M. Ch. Bonaparte et de Richard et Swainson.

Trouvée aux Gallapagos par M. Néboux.

FAUVETTE DE WILSON.

Erythaca Wilsonii, Swains.

Sialia - id.

Motacilla sialis, Linn. Wils., Am. or., pl. 5, fig. 5.

Enanthe - Vieill.

Saxicola - Ch. Bonap.

Se rencontre jusqu'a l'est des montignes Rochenses d'après Richard et Swainson; trouvée à Monterey, Haute-Californie, limite extrime de son habitation occidentale, par le docteur Néboux.

\section{PLANCHE III.}

\section{GRALLARIE SQUAMMIGÉRE.}

Grallaria squammigera (FI. Prévost et O. des Murs).

Crar. spec. Gral. Suprd olivascente schistacea unicolor, plumis fusco vix conspicuè marginatis; subtùs lateribusque colli et loris ochracea, plumis totis ante apicem maculà squamaformi nigret notatis; gutture et collo antico albis aut pallidè ochraceis utrinque viltâ nigra limbatis. Tibiis grisescentibus, pedibus pallidè rubro-brunneis (de La Fresnaye, Rev. zool., novemlıre 1842, p. 553).

Description. Celfe nourelle espèce de Grallarie, remarquable par sa taillo de heaucoup plus forte que celle des Grallaria rex el imperator, a la coloration générale de tous ses congénères. 
Elle a le sommet de la tête, la nuque el le derrière du cou gris de fer légèrement écaillé de noirâtre; les épaules, le dos, le manteau, les scapulaires et le croupion brun-olivâtre; les grandes couvertures lisérées finement de fauve à leur extrémité; les rémiges et les rectrices couleur du tere d'ombre beancoup plus paile sur leur tranche extérieure; le front, les lorums, les parties latérales du cou, l'estomac, la poitrine et le

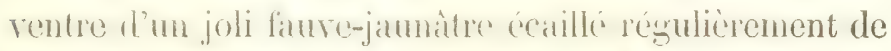
brun-noiràtre sur chaque: plune; les couvertures inférieures de la queue fauves, sans taches; le menton, la gorge et le devant du cou blancs, ce blanc encadré de deux traits noirs partant de chaque côté du menton; bec couleur de corne; pieds d'un brun-rougcaitre. Màle adulte.)

Lat femelle ne differe dı màle que par l'absence de blanc à la gorge.

Longueur totale. ..... 25 centimètres.

- destarses. ..... 06

Habit. Santa-Fé de Bogota en Colombie.

\section{PLANCHE IV.}

\section{GRALLARIE DE GUATEMALA.}

Grallaria Guatemalensis (Flor. Prévost et O. des Murs).

Cins. spec. G. suprò brunnea; subtìs rufescens; pectoris maculis aliquol semi-collaribus nigris (de La Fresnaye, Rev. zool., 1842, p. 534).

Il existe une telle aflinité de couleur entre cette espèce et la précédente, que nous arons hésité longtemps à 
les diflérencier l'une de l'autre, pensant que la plus petite pourait bien n'ètre que le jeune de la plus grande. Mais un examen approfondi nous a bientòt convaincus que si rapprochée et si uniforme, en quelque sorte, qu'en étais la P'tilose, elles devaient constituer deux esprees bien distinctes, rien, daus colle.ci, nindiquant. trace du moindre caractère propre au jeune âge.

En voici au surplus la description. Description : Tête et derrière du cou gris de fer, chaque plume cerclée régulièrement de noir; dos, manteau et couvertures alaires brun olivitre écaille légrement de noir ; rémignes secondaires brunes; rémiges primaires et rectrices rousses; paupières blanchâtres; tout le dessous du corps, depuis le menton jusqu'aux couvertures inférieures de la queue d'un faure roussàtre, les plumes de la gorge offrant quelques traits écailleux noiràtres.

Longueur totale. . . . . . 16 centimètres.

- des tarses...... 05

Habit. Guatémala.

\section{CYANOCORAX DE SAN-BLAS.}

Cyanocorax San-Blasianus (J.-B. Gray.); Gen. of B., 1845. Pica San-Blasiana (de La Fresnaye), Rev.zool, 1840, p. 3z3, Mag. de zool., 1842 , Ois., pl. 28.

Crars. spec. C. Suprà ultramarino-carulescenti, sublùs nigrescenti-indigotino, crista frontali nigra, singulis plumis apice revoluto ceruleis, collo capiteque nigris, rostro albo nigroque semi-partito, pedibus nigris.

Cet Oiseau, qui fait partie des belles espèces du Voyage de la Vénus, a été décrit ainsi dlans la Rev. 
zool. d'octob. 1840, pag. 290, par le docteur Néboux, qui lui a donné le nom de Geai de San-Blas :

«Bec blanc mêlé de noir. Huppe noire; les extré" mités des plumes ayant une teinte bleue. Tète et cou ") noirs. Manteau bleı, ventre noir nuancé d'indigo. "Ailes d'un bleu plus clair que le manteau en dessus y et grises en dessous. Queue bleu de Prusse. Tarses "noirs.

„ Longueur totale. . . . . 23" centimètres. "

Irabit. San-Blas (Mexi(que). M. Leiclancher en a rap)porté des individus d'Acapulco. "Elle y vit, dit-il, " (lians ses notes publiées Rer. zool. nov. 1840, page „323) en troupes, et j'aurais pu en tuer cinquante " dans un jour, au moyen d'un Pic blessi que je faisais ") crıer et qui, par ses cris, les attirait en grand nom) bre ainsi qu'une foule d'oiseaux, tels que Pics, "Perruches, etc. "

La tigure en fut publiée sur un individu provenant de l'Expédition de la Vénus, dans le Magas. de zool. 184.2, pl. 28, par M. de La Fresnaye qui l'accompagna de la description et des observations suivantes :

"Cet oiseau, d'après la forme de son bec, appar" tient plutôt au groupe des Corvus, que l'on est " convenu de désigner par le nom de Pie, qu'à celui ") des Geais proprement dits à bee plus faible. Son bec ") est mème si grand, à proportion de sa taille assez " petite, qu'on serait tenté, d'après ce Caractère, ) comme aussi l'habitude de vivre en bandes, olsservée " par M. Léclancher, de le ranger avec les Corneilles, ) si la brièveté de ses ailes ne s'y opposait. 
D) De la taille de la Pie geng de Temminck, pl. col. 169, 1) son bec est plus allongé au moins d'un quart, la tran") che supérieure entre assez avant entre les plumes du n) front, la courbure en est prolongée et n'est pas plus „) sensible à l'extrémité que dans le reste de la lon"gueur; il paraît avoir été d'un blanc bleuâtre avec la ") base et la pointe couleur de plomb. La tête, le cou en ») entier, ainsi que tout le dessous, sont d'un noir pro" fond; de la base du bec s'ćlève un faisceau de plumes " étroites en partie décomposées, formant une huppe „) frontale recourbéc en avant à son extrémité, laute de " 10 lignes; elle est noire dans notre individu, et légè") rement terminée de bleu dans celui décrit par M. Né"boux dans la Revue zoologique; toutes les plumes du ns sommet de la tête sont également terminées de bleu ") sombre. Le dos en entier, le croupion et les scapun) laires sont d'un bleu d'outremer, la queue est d'un n) beau bleu de Prusse plus foncé, elle est de longueur

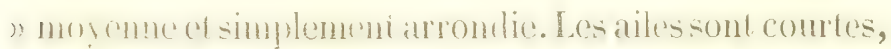
n s'étendant à peine au tiers de sa longueur et sont d'un ") bleu vert de mer. Les couvertures inférieures de la " quear sont nuancres du mime bleu qu'elle. Les pattes ") paraissent plombées.

"Longueur du bec, depuis son ouverture, 4 centi") mètres." 


\section{CYANOCORAX DE BEECHEY.}

Cyanocorax Beecheii (G.-B., Gray), Gen. of B., mars 1845.

Pica Beecheii (Vig.), Zool. journ., t. 4, p. 555, Zool. of Beechey's voy., pl. 22, p. 6.

Corvus (pica) Beecheii (Eyd. et Gerv.), Mag. de zool., 1857 , Ois., pl. 20 .

L'individu dicrit primitivement par Tigors avait éte rapporté par l's yortition de Brechey de l'ile de Montréal. Le nôtre, comme celui de M. Botta, figuré par

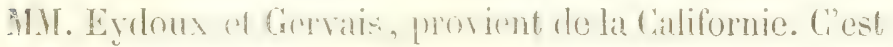
à Monterey que M. Néboux l'a pris.

\section{CASSE-NOIX.}

Nucifraga caryocatactes, Briss.

Corvus - Linn., Pl. enl. 50.

Nucifraga guttata, Vieill., Gal., pl. 150.

Consideré pendant Innglemps comme particuliog a

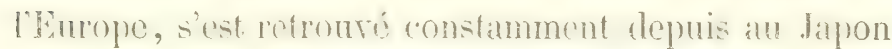
et au Kamtschaka. Le docteur Néboux en a rapporté

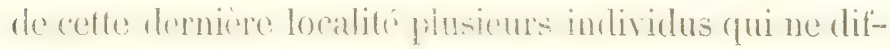
fèrent aucunement de ceux d'Europe.

\section{ETOURNEAU MILITAIRE.}

Sturnella militaris, Vicill.

Sturnus

$$
\text { Gmel., } P l . e n l .115 .
$$

Cet Oiscau, considéré juscpu'i) co moment romme ap)partenant exclusivement au Continent Méridional de l'Amérique, n’avait jamais été supposé devoir faire d'excursions vers le Ciontinent septentrional; la sirience 
du moins ne l'avaii fals encore constate. Les Naturalistes Iu bearle ont seulenent reconnu dernierement sa trace sur lat còte orientale du Detroit de Magellan et des iles Malouines.

C'est un fait assez curieux que la présence de cette "spice assonticllement meridionale en Californic d'ou alle a éte rapportie de. Monterey par le docteur Néboux, car cet Oiseau ne Rigure pas sur la table de Richard et

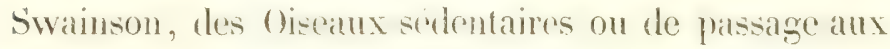
États-Unis.

\section{BEC-CROISÉ LEUCOPTERRE.}

Loxia leucoptera, Gmel. Vieill., Gal., pl. 52.

- falcirostra, Lath.

Curvirostra leucoptera, Wils, sm. or., pl. 51, fig. 5.

Pitiopsistacus Americanus.

Carvirostra leucoptera, Daud.

Commun dans toute l'Amérique du Nord. Rapporté de Mlonterey par le docteur Néboux.

\section{CACTORNIS GRIMPEUR.}

Cactornis scandens (Gould.), Proc. zool.'soc., 1857, Zool. of the Beagle, p. 104, pl. 42.

C. ... intensè fuliginosa, crisso albo; rostro et pedibus nigrescenti-brunneis.

Fam.-- Corpore superiore, gutture pectoreque intense brunneis, singulis plumis pallidiore marginatis; abdomine crissoque cinereis, stramineo tinctis; rostro pallidè fusco; pedibus nigrescenti-fuscis (Gould, loc. cit.).

Cet Oiseau, nommé par le docteur Néboux Tisserin des Gallapagos dans la Rev. zool. d'octobre 1840, 
page 291, y a été decrit anci par lui d’apres un indivodu qu'il a rapporté :

( Corps en général brun-noirâtre, chaque plume est "légèrement hordée de blane, surtout soun le ventere ") et en avancant vers la région anale; mandibule supr) rieure à arête arrondie, très-pointue et noire; mandi"bule inférieure jaunâtre, la mandibule supérieure de" passant l'inférieure de deux millimètres environ.

" Longueur totale. . . . . .

" - de la quene. .. 04 centimètres.

" - du bec.... 18 millimètres.

"Ilabit. Ile St-Charles (Archipel des (ialilapagos). " Cet individu est un jeune màle prèt à prendre la livrée d'arlulte, ainsi que l'indique le brun foncris de sa couleur générale, et surtout la couleur de son bec, qui est dojaj celle du mâle adulte. Celui-ci est entièrement d'un noir intense avec les rémiges primaires brunitres et la région anale d'un blanc cendré.

Dans la Revue zoologique du mois de novembre de la mème amnée 184.0, para 32.3 , H. de La Fresnaye, à qui M. Léclancher avait communiqué un autre inclividu de cette espéce, publia à son sujet les observations sujvantes que nous croyons deroir ciler tout entieres parce qu'elles expriment ei résument alussi bien et mieux que? nous ne le pourrious faire ce que nous arions à dire sur cet Oiseau.

"Nous avons, dit notre Ornithologiste, quelque » doute que l'Oiscau décrit sous ce nom par M. Néboux "s soit réellement un Tisserin, et nous serions plutòt " porté à croire, d'apris la description dr son plumage 
n) Irun-noirâtre aree wangu plume bordere de blane, ") d'après la brièveté de sa queue longue seulement » de 4 centimètres, chez un Conirostre à bee long de " 18 millimètres, qu’il doil faire partie de ce nouveau ") groupe de Granivores marcheurs, recucillis aux Gal" lapagos par les naturalistes anglais du Beagle, que - M. Gould a désignés et décrits sous le nom de Geo. ) Spiza dans les Proceedings $183 \%$, pag. 5 et 49 , et forn mant douze espèces différentes, remarquables toutes " par une forme courte et ramassée, par une queue fort ") courte, par des ailes obtuses et arrondies, par des ") pieds d'Oiscaux marcheurs, à ongles peu arqués, par " un plumage noir on noirâtre chez les mâles, moins ) obscur chez les femelles, dont les plumes sont bor» dées de cendré on de roussâtre, on d'olivâtre, et par ") l'habitude de se tenir en grandes bandes à terre, où „ ils se nourrissent de graines de gramincées dont il y a ") ample récolte dans ces îles. M. Darwin, le naturaliste " de l'expédition, ajoute qu'ils sont si peu farouches " qu'on n'a pas besoin de fusil pour s'en procurer. Ils ") sont encore remarquables en ce que les douze espèces ») décrites par M. Gould, et dont cunđ sont déjà figurées „) dans le Beagle's Toyagye, tout en réunissant les carac" tères communs indiqués ci-dessus, diffèrent entre "n elles par la forme du bec présentant chez quelques") unes l'énormité d'un bec de Coccothraustes le plus vo») Iuminenx, et se dégradant jusqu'i celle d'un Pinson ") ou d'un Ignicolor, en se comprimant et s'allongeant ") chez quelques autres, ce qui lo fait alor's ressembler à ") $x$ bec de Tisserin ou plutôt d'Euplectes. 
"Nous avons dans les Oiseaux de M. Léclancher ") une femelle appartenant à ce groupe et à la petite di") vision à bec long et comprimé, clésignće par M. Gould ") sous le nom de Cactornis. M. Léclancher me dit à pro") pos de cet oiseau : Il vicnt des Gallapagos; j'y cn ai tué ) un autre à plus gros bec, mais je ne sais ce qu'il est de") venu. Presque tonjours ì terre, ils sont si peu farouches "qu'on en a tué plusieurs à coups de baguettes de fusil. "Les espèces de ce genre qui étaicnt à bord ont été re" mises par MII. Néboux et Filleux an Muséum.

"A ces divers détails qui m’ont fait soupconner que ") le Tisserin décrit par M. Néboux était un de ces Géo-

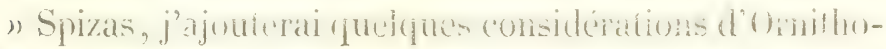
n) logie géographique.

"Les Gallapagos, peu éloignés du Continent Améris cain, n'ont encore fourni, tant aux explorateurs du "Beagle qu'ì ceux de la Ténus, qu'une Ornithologie tout 3. américaine quant aux genres; des Moqueurs, des " Gobe-Houches rubins, des Effrayes, ct enfin ce nouveau "groupe de Gro-Spiza, et on n'y a trouvé aucun genre ") particulier à l'Ancien Continent. La présence du genre " Tisserin, dont on ne connaìt aucune espèce améri") caine dans ces îles, serait donc un fait tout nouveau n et qui ne serait pas sans importance. Nous invitons "donc M. Néboux à comparer l'Oiseau qu'il décrit " comme Tisserin, avee les descriptions des différentes ") espèces de Gréo-Spizas de M. Gould, tant dans les Pro" ceedings, 1837, que dans lo Beagle's Voyage, où cinq ") sont figurcés, et à vouloir bien publier de nouveau le "résultat de ses recherches qui sera loin d'être indiffé- 
"rent pour la science et pour l'Ornithologie géogra") phique.

" Quoique les atuteurs anglais regardent ce groupe de " Géo-spiza comme particulier aux Gallapagos, nous " avons la conviction que le Continent Américain doit " renfermer quelques esperes analogues au milieu de " ses nombrenx Conirostren, et nous croyons déjat entre") voir quelque analogie de couleur et de forme de bec, ") entre le sous-genre Cactornis et l'Oiseau connu sous "le nom de Père-Noir de la Martinique."

\section{GEOSPIZA FULIGINEUX.}

Geospiza fuliginosa (Gould.), Zool. Beagl., p. 101.

Cirar. spec, G. (ITas) intensè nigro-fuliginosa, tegminibus cauda inferioribus apice, remigibus rectricibusque lateraliter albido-marginatis, rostro ingro, pedibus nigris.

1.'individu dont M. Gould a liait le y yoe de cette espece n'était pas adulte, ainsi qu'on peut en juger d'après la diagnose suivante qu'il en a donnée :

G. (mas) intense fuliginosa, crisso albo, rostro fusco, pedibus nigrescentifuscis.

Un individu se rapportant évidemment à la même espece, rapporté par la Téms et domne au Musémm de Paris par II. Filleux, differe de celui décrit par . I. (iould, en ce que tout le corps est, non pas fuligineux, mais d'un noir assez intense, mais à reflets brunâtres; la région anale est du même ton que le reste du corps; seulement les couvertures inférieures de la queue sont finement corclées de gris blanchatre; la mòme teinte 
borde les rémiges et ces rectrices; les primamres sont brunâtres, et le bec et les pattes sont d'un noir pur. Le dessous de la quene est grisitre, rubané transversalement et d'une maniere assez réguliere de stries d'une teinte plus foncée.

C'est sous ce rapport que nous avons cru devoir refaire, en la complétant, la diagnose latine du naturaliste anglais.

Voici celle qu'il a donnée de la femelle que nous ne connaissons pas.

Fomina : summo corpore, alis, caudarpue intensè fuscis; singlilis plumis cinerascenti-ferrugineo marginatis; corpore infra cinereo; sinqulis plumis medium versus obscurioribus; rostro brumneo; pedibus nigrescenti-brunneis. Dimensions de notre individu:

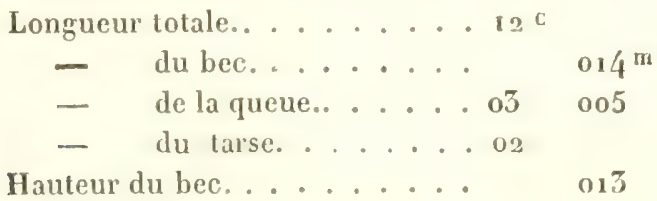

Habit. Les Gallapagos.

\section{GUIRACA CENDRF.}

Guiraca cinerea (de La Fresnaye), Mag. de zool., 1845, Ois., pl. 50 .

Camarhynchus cineraceus (G.-B. Gray), juin 1844.

Cinar. spec. G. - Suprà dilutè cinerea; fronte basique rostri nigris, subtùs cinereo-albida, gutture et collo anteriore albis, rostro flavo, pedibus tantùm flavidis.

Ce Gros-Bec, que l'expédition de la Vénus doit revendiquer au nombre de ses découvertes, a été décrit 
sur un individu à lui communiqué par .11. Léclancher, par II. de La Fresnaye qui en a publié la figrure dans le Mag. de Zool. 181.3, ois., pl. 30, en en faisinnt l'objet de l'article suivant que nous nous bornerons à reproduire :

* Swainson, dit l'habile (ornithologristo, designa par * le nom générique de Guiruca, qu'il cluangea ensuite en " celui de Coccoborus, une partie des Gros-becs d'Amé" rique. Les espèces types sont : le Gros-bec blen des "Etats-Unis, le Gros-bec azulam du Brésil et le Gros-bec "rose-gorge, qui, réellement, diflèrent assez de notre "Coccolhraustes d'linrope pour avoir autorisé cette se" paration.

„) Notre nouvelle espèce américaine, rapportée des n iles Gallapagos par. N. Lechancher, alors chirurgien de "La Vénus, offre, dans la forme de son bec el de ses " pattes, des modifications assez distinctes du genre " Guiraca pour qu'on puisse l'en séparer à son tour, au ") moins comme sous-genre, surtout si, à ces caractères ") différentiels, il s'en joint aussi (quelqu'un dans les han bitudes. Ce bec, effectivement aussi élevé que celui n des Guiracas, en diffère en ce qu'il est très-arqué en ") dessus et beaucoup plus comprimé; et ne peut guère " être comparé, parmi les espèces américaines, qu'à " celui du Père noir (Fringilla noctis), espèce toute "noire, qui a le devant dn cou et des yeux roux-can"nelle, tandis que les paltes, très-robustes, à ongles " courts, mais larges et fortement courbés, n'ont guère n) d'analogues parmi les fros-bees americains, mais "bien chez le genre Psitarin des Sandwich. On peut 
dire, entin, que notre nouvelle espece est un lirus-bee

"Guiraca à bee de Pére-noir el à pattes de P'sittacin.

"Il se rencontre dija, dans la famille Tamagridée d'A. ") mérique, quelques especes à pattes remarquablennent n) fortes, comme chez notre oiseau, et à bec aussi com") primé quoique moins élevé, ce sont, 1º l'Esclave des "palmiers (Vieil. Gul., pl. 146); le Tanargra rubrigularis „) ou rubricollis (Spix, pl. 气6), dont Swainson a fait n son genre Lamprotes, at le Tanagra ruficollis (Licht.), n) Tangara hirondelle (Lesson., Tr.) dont cet auteur fait n) son genre Cigpsnayra, et Swainson celui de Leucopygia. "Il est certain que, si ces trois oiseaux presentent, dans n leurs habitudes comme dans la grosseur de leurs " pattes, quelques caracteres particulicrs qui en scraient "la conséquence et différeraient de cend des autres ) Tanagridées, leur formalion en genres distincts de ce" lui de Tachyphone, auquel ils semblacient appartenir, n) serait fondec, de mème que celle de notre Giros-bee à ") bec comprimé et à paltes de Psiltacin le serait aussi, ) si les mèmes différences de mocurs avaient lieu entre n lui ef les autres Guiracas d'Amérique. Je proposerais m) alors, pour nom de genre, dont il deviendrait le type, n celui de Piezorhina (bec comprimé).

„Le Guiraca cinerea, de la grosseur, à peu près, du "Gros-bec bleu des blats-Lnis, a la queue beaucoup " plus courte et presque carrée, les ailes plus longues » et s'étendant jusqu'au delà de la moitié de celle-ci; ") les tarses, les doigh et les ongles tris-rubustes et d'un ") blanc jaunâtre; le bec élevé, très-arqué en dessus, " comprimé, aver les narines petites, mondes pereés 
n) à égale distance de sa partie supérieure, et de sa ") commissure. conte commissure très-sinueuse a sa base ») et à son extrémité; il est partout d'un jaune assez vif. "'Tout le dessus de l'oiseau est d'un cendré peu foncé. ) Le front et le tour du bec, excepté en dessous, sont ) noirâtres. La gorge et le devant du cou sont blancs. ") Tout le dessous est d'un cendré blanchâtre, avec les ; flancs un peu plus foncés, l'abdomen et l'anus pres》 que blancs. Les rémiges et les rectrices sont gris-ar") doise, finement bordées de blanc-grisâtre.

") Longueur totale, 1 "s centimètres.

"Cette espèce a été tuée aux îles Gallapagos."

PLANGHE V.-FIG. 1.

TANGARA A NUUUE ROUSSE.

Tanagra (Calliste) rufivertex (Fl. Prévost et O. des Murs).

Cinar. SPEC. Tan. capite nigro, singulis plumis apice violaceo nitentibus, verlicis exceptis apice rufis; corpore toto cobalto cerulescenti; pallio malachite virescenti cobalto squamulato; remigibus rectricibusque nigris margine concoloribus; crisso fulvo-albido; rostro et pedibus nigris.

Ce Tangara, sinon par la similitude des tons au moins par l'exacte et semblable distribution de ses couleurs sur fond bleu, rappelle un peu le Procnopis atrocoerulea (Thchud.), Consp. av. n. 128, el Faun. Per. Vog. Taf. 13, fig. 2; sauf la distinction générique, et à lexreption que chez celui-ci, c'est le blen-noirâtre qui donine, tandis que, chez notre Oiseau, c'est le bleuclair mat ou cobalt, tournant parfois au malachite.

Description: Tète et hase du bec noires, les plumes de 
la base du front, de la nuque et du derrière du cou terminées d'indigo tournant au violet brillant; celles du sommet de la tête terminées de roux; du reste en entier d'un bleu-cobalt mat et sans reflet; toutes les plumes du croupion et du dessous du corps cilićes et déconposées; les autres au contraire squamenses et bien distinctes; manteau vert malachite, chaque plume liscrée finement de cobalt; rémiges et rectrices noires, les premières jusqu'aux deux tiers de leur longueur, les dernières dans toute leur étendue largement liserées sur leur tranche extérieure de cotte derniere couleur; les rémiges secondaires entierement noires lans leur page interne, bleu-cobalt dans celle externe, croupion et cuisses d'un fauve léger : bec et pieds noirs.

Longueur totale. . . . . $141 / 2$ centimètres.

Hubit. Guatemala.

$$
\text { PLANCHE V.- Fig. 2. }
$$

\section{TANGARA LABRADOR.}

Tanagra Labradorides (Boissonneau), Rev。 zool. 1840, p. 67. Caliste (J. R. Gray).

Char. spec. Tan. suprà viridi-aurato, lumine Labradorensis lapidis splendenti; rostri basi, capite summo, colloque superno, ac scapularibus, remigibus, rectricibusque nigris; illis caruleo virescenti tenuissime marginatis; flexurci alarum cceruleà; tegminibus minoribus splendide viridibus; crisso cruribusque auratè rufis.

Nous nous bornerons à transcrire ici la description donnée de cet Oiseau par celui qui le premier l'a fait connaitre dans la Revue zoologique de 1840, page 67.

"Cette jolie espèce, dit M. Boissonneau, a tout le 
" corps dessus det dessons d'un vert doré à reflets bleus net or qui rappellent tout à fait les reflets de la pierre » de Labrartor ou Foldspath chatoyant. Le devant des " yeux, le bord du front, ainsi que le menton sont „) noirs. Cne larese bande de rette couleur part du vertex ») el se prolonge en arrière sur toute la partic supérieure » du cou. Les ailes et la queue sont noires avec leurs ") pennes firement bordies de vert. Le poignet de l'aile nest d'un bleu passant au vert brillant sur toutes les "petites couvertures. Ies couvertures inféricures de n) l'aile sont d'un blanc légrement orracé, ainsi que le s milieu de l'abdomen qui prend une teinte d'ocre plus "prononcée vers l'anus, sur les couvertures inférieures 3) dela queuect sur les jambes. Bec noir; pattes brunâtres. ") Longueur totale, 13 centimètres." Habit. Santa-Fé de Bogota.

\section{PASSERINE DE LÉCLANCHER.}

Passerina (Spiza) Lrclancherii (de La Fresnaye), Rev. zool., 1840 , p. 260; MTag. de zool, 1841 , Ois, pl. 22.

Calliste Leclancherii (G. R. Gray), Gen., juillet 1844.

Cran. spec. Pass. suprì corulea capite dorsoque olivascentibus; subtìs junquillacea, pectore aurantiaco; alis et caudd nigrescentibus, viridi ccrulescenti marginatis; rostro corneo, pedibus plunecis.

Nous reproduirons encore pour celte espèce, dont deux individus ont che rappostés par la l'énus, la description détaillex spu'on a publice le premier M. de La Fresnaye, dans la Rev. zool. 1840, page 260.

"Cette charmante pelite espèce. dit-il, au plumage "Is plus suave doif atre classéc dans les Passerinés de 
„Vieillot, ou Spiza, Bonaparte, et dans le petit groupe " que ce savant a drisigne par le nom de Spizas-Tanagras, " et qui renferme, outre les especes nommés le Pape ") te le Ministre, les Spiza amana of versicolor de cet Aun) teur. La nôtre est donc la cinquième espèce de ce "petit sroupe de transition des Fringilles aux Tangaras, n) genre qu'il rappelle non-seulement par l'agréable vab) rietc de lat coloration, mais aussi par l'echanerure du " bee, qui se remarque chez quelques espèces, telles v que le Spiza amana et notre espèce nouvelle.

„ Cellc-ci, de la taille à peu près de la Passerme Pape, n a le dessus de la tête olive, tout le dessus du corps, 》) les joues, les côlés du cou et les couvertures des ailes m) d'un joli bleu de ciel méle d'olive sur le milieu du dos ») et sur la nuque. Les ailes et la queue sont noirâtres, " mais toutes les pennes sont hordées de bleu verdatre. "Tout le dessous est du plus heau jaune jonquille, pre3) nant sur la poitrine une nuance orangée très-vive, "puis se digradant insensiblenent en jaunc-serin vers "l'abdomen. Les lorums et le tour des yeux sont jaunes. "Io bec est couleur de corne arec la inandibules supé" rieure légèrement échancrée. Les pieds sont plombés.

b) Habit. Tuée près d'Acapulco, au Mexique.

3) Deux seuls individus de cette jolie espèce ont été n) rapportés par la Térius, dont un tué par M. Léclancher. „Cet Oiscau se tient habituellement dans des lrous. 1) sailles épineuses ressemblant à notre épine noire, et ") où il est très-difficile de l'apercevoir. On troure aussi " sur le trone du mème arluste un hean Bulime blane "zébré de violet. 
"Lihabitation de cet Oiseau, dans les buissons four. ") rés et dans les vallées, comme chez les Passerines "Pape et Ministre, est une conformité de mœurs bien ") remarquable entre ces trois espèces, el qui, malggré " leurs rapports de coloration, les éloigne des Tangaras " qui se tiennent habituellement à rlécouvert et se per") chent sur le sommet des arbres des forêts. ")

\section{PLANCHE VI.}

\section{BRUANT A DOUBLE CROISSANT.}

Emberiza biarcuata (Fl. Prévost et 0 . des Murs). Fringilla - - (Fl. Prévost et 0. des Murs), Allas, pl. 6. Arremon biarcuatus (J.-B. Gray), Gener, 1849, Append.

Char. Spec. E. - Suprà fuliginosè brunnea parumper olivascens, fronte, genisque nigris, regione periophthalmicd albd rufo circumcincta, vertice et collo posteriore rufis: subtus alba, lateraliter cinerascens, crisso ochraceo, remigibus recfricibusque orimnco-nigrescentibus, primis cinereo marginatis, rostro nigro, pedibus brunneis.

Cic Fringille, désigné sur notre planche VI sous le nom générique de Moineau, est un véritable Bruant. Ainsi la commissure des deux mandibules, au lieu de ne former qu'une ligne plus ou moins courbe ou infléchie, forme une ligne brisé à angle obtus vers son ouverture; de plus la mandibule supérieure porte cette protubérance mterne si caractéristique chez les vrais Bruants. Toutefois les caractires des pattes different un peu de ceux assigrés à ce genre : elles sont ici trèsfortes, les ongles sont également forts et tres-arqués, l'mugle meme du poure est te la longueur de ce doigt 
ef très-arqué. Par ce dernier caractère il se rapprocherait des Plectrophanes, à part la courbure de l'ongle. On peut même dire que, n'était la forme du bec, qui est celle si spéciale aux Bruants, ce serait un de ces Tangaras à couleur sombre, dans le gemre du Tanagra ruficollis de Spix, Av. Bras., 2) vol., tab. 53, f. 3, ayant le plus grand rapport avec celle des Bruants.

Description. Celui-ci a le milien du front et le bas de la joue noirs; une tache superciliaire partant des narines, la région periophthalmique, le menton, les bords inférieurs de la joue, la gorge, le cou et le milıeu de l'abdomen blanes; le dessus de la lète, la nuque et le haut postérieur du cou d'un roux-marron, ce roux encadrant la région periophthalmique et se continuant jusqu'au méat auditif; le bas postérieur et les côtés du cou, ainsi que ceux de la poitrine, sont d'un griscendré; le dessis du corps et de la queue d'un brun enfumé légèrement olivìtre; l'anus et les couvertures caudales inféricures d'une teinte ocracée; les rémiges et les rectrices d'un Jorun-noiràtre, les prenières finement bordées en grisàtre; les grandes couvertures alaires hordćes de roussâtre; la queue est arrondie, le bec noir, les tarses sont bruns.

Longueur totale....... 16 centimètres.

- de la queue. . . . . . ${ }^{2} 1 / 2$

Hab. Liindividu que nous avons figuré, sur un exemplaire dont le bec était incomplet, a été rapporté de la Californie par le docteur Neboux; celui qui a servi à notre descruption vient de Guatémala. 


\section{SUR LE GENRE PTILONOPE,}

ET LA DIVISION A Y INTRODUIRE.

On sait que le genre P'tilonopus, créé par Stwainson (Zool. Joumal, vol. I, p. 473, ann. 1824-1820̈), a été adopte par fous les Ornitholocistes, et respecte par M. G.-R. Ciray, gui l'a comerercidans son Generu of Birds.

Or, ce genre a été destiné par son auteur à renfermer les colombides oflant pour lous canadires des ailes médiocres, à première rémige très-brève, la plus courte, et tellement richancrese interieurement qu'clle en deviont courbe ef prestume falciforme; à bees grèles et à tarses presque entiermont emplumés. Il le caractérisait en effet dans les termes suivants :

Ala mediocres, remigum prima apicem versus contracti, eertid quarlatue longissimis;

Rostrum gracile;

Tarsi plumosi.

Et il domait pour type do ces genre ce qu'il considérait comme une simple variété, nomméc par lui Vorietas Rerjina, du Ptilomopus purpuratus, Columba purpuratu, de Gmelin et Latham, c'est-à-dire du Kurukuru de Temminels, la plus anciennement connue de toutes les espèces de ce genre.

A prendre dans les termes de la Caractéristique de Swainson, nul doute que son genre Ptilonopus, tel que I'a composé M. G.-R. Gray, ne soit rigoureusement exact. Vais on remarquera rue parmi les especes qui 
y figurent, il en est qui possèdent seules un caractère unique et des plus tranchés dlans la ronformalion de leurs plumes pectorales, caractère véritablement suffisant pour en constituer un genre à part.

Nous pensons lone que le genre Ptilonopus demanderait, pour plus d'exactitude et de clàté, à ètre divisé en deux parties.

La premiere comprendrait les vrais Ptilonopes, tels que les a definis l'ornithologiste anglais, et conserverait son nom générique de Ptilonopus.

Et il conviendrait de composer la deuxième des espèces qui, aux caractères assignés aux Ptilonopes, joindraient re caractere si singrulier que présente notamment le Kuruhuru, Columbu peirpurute, dans la ptilose de sa région pectorale; nous voulons parler de la forme des plumes de cette partie, qui présentent toutes l'aspect de plumes dont le rachis serail coupé à peu de distance de sa pointe, qui se trourerait ainsi dépassée par la longueur des harbules do droite et de gauche, et ne ligurerait plus alors qu'un Trenverse' 1 , dont l'extrémité subsistante du rachis serait le sommet. Il est mème remarquahle que to nombre de ces espèces ne laisse pas que d'etre considerable, et comprend pres de la moitié de celles raiggées jusqu'à présent dans le genre Ptilonopus, toutes especes appartenant exclusivement aux archipels de la mer du Sirl. Cette seconde partio prendrait pour nom gencerique celui de Kurukuru, inposé par M. Temminck à celles des espèces les plus anciennement connues qu'il ait ligures te premicr, et serait caractérisée ainsi : 


\section{Genre KURUKURU.}

Plumes du cou ef de la poitrine roides, et échancrées à leur extrémité qui est bifide;

Ailes médiocres, la premiere rémige très-brève, et falcıforme la plus courte, les troisième et quatrième les plus longues;

Bec grêle;

Tarses à demi ou presque entièrement enplumés.

\section{Genus kURUkURU.}

Plume rolli pectorisque rigide apice bifida vel furcate: Ala mediocres, remigum primâ apicem versus contracta, tertiâ quartâque longissimis;

Rostrum gracile;

Tarsi plumosi.

Quant aux espèces qui nous paraissent devoir entrer dans la composition de ce grenre, nous nous réserrons de les indiquer après que nous aurons passé en revue les individus, à notre connaissance, qui doivent ètre considérés comme appartenant réellement au Ptilonopus (Columba) purpuraus, et ceux qui en ont été ou doivent. en être entièrement distingués.

Ainsi, de tout temps, depuis Gmelim et Latham, jusqu'à ces dernières années, il est constant que l'on a confondu et que l'on confond encore avec le vrai Pt. purpuratus, et comme variétés de celte espece, des individus dont on a, avec raison, fait depuis des es- 
pèces distinctes, parce qu'elles en diffèrent essentiellement, et que les observations successives des voyageurs, de mème que les études des Ornithologistes ont démontré jusqu'à l'évirlence la raison de ces différences.

Ces variétés sont en effet si nombreuses que ce ne pouvait être qu'a force de temps et d'observation qu'on devait espérer arriver à les débrouiller. Aussi est-ce avec quelque apparence de raison que Lesson a pu dire, ¿ l'époque oi il décrivit sa Col. Kuruliuru, variété Taïtensis (Voy. de la Coquille, 1 vol., p. 297, ann. 1824), que : "Cette Col. Kurukuru, qui se troure clans toutes "Ies iles de la Malaisie et de l'Océanie, depuis les Mol"luques, les Philippines et les Mariannes, jusqu'aux "Sandwich el aux îles de la Société, et qui, en tout n) lieu, identique par l'ensemble de ses formes et les n) masses de couleur de son plumage, ofire partout des "nuances si variées, qu'elles ont déjà cent fois torturé " les naturalistes systématiques, aux définitions précises ") desquels elle semble vouloir échapper. ")

Il est vrai que vingt-cinq ans après, c'est-à-dire en 184.7, instruit par l'expérience et par les progrès de l'Ornithologie, auxquels il avait donné lui-mème un si puissant concours, il exprimait une opinion différente et plus exacte, à l'occasion de la description de son Ptil. Emilice, qu'il reconnaissait ètre le mème que notre Kurularu Dupetit-Thouarsii, et plus conforme à la vérité, en disant, à propos du groupe des Ptilonopes auquel il rapportait cette Colombe, que « comme chaque ) archipel a des variétés constantes, force est de les n) décrire comme espèces distinctes. n 
C'est pour bien laine sentir l'importance de ces distinctions que nous ayons entrepris le travail qui suit, et pour vulgariser le résultat des travaux de nos prédécesseurs, tels que MIN. Swainson et Gould, toujours excellents par leurs résultats, mais manquant en général de cet esprit d'analyse qui, en établissant une distinction entre drux ternes de comparaison, foumit les movens de controbe indispensables à tout observateur, pour bien sabis la ration des rapports ou des différences qui presifue tonjours précedent el devatent accompagner l'ibhlisicment el la création despèces nouvelles en histoire naturelle.

Cela posé, nous allons, si ingrat que soit le travail dont nous parlons, puisqu il ne loil consister en grande partie qu'en ritations of an descriptions, nous allons passer en revue les diverses descriptions domées du Pt. parpuratus, comme tgpe du nous eau genre kivrutiuru que nous proposons l'etahlir, el indiquer les espéces qui doivent entrer dans ce genre.

\section{KURUKURU A COURONNE POURPREE.}

Kurukuru purpuratus, O. Desmurs et Flor, Prévot.

Columba purpurata, Loth., Gmel.

Ptilonopus purpuralus, Swains.

Columba viridissima, Tunn.

Spec. Char. K. viridis, sublus in cinereum vergens, fronte et verlicis parle purpureis, crisso flavo, remigibus viridi nigrescentibus, remiges primores prater duas primas, margine flave, rectrices aquales, subovalo, margine anteriori virides, fascia interrupta grised notata.

Comme toutes les espèces exotiques anciennement 
comnues, le Kurukuru à couronne pourprese, quant à son véritable type, a fini par être confondu avec ses varich congeneres plus ou moins rédles, à tel point que chaque auteur ayant eu son type particulier, il devient assez difficile de se fixer sur la variété, ou soidisant telle, qui doit definitivement dtre prise pour le type de l'espèce.

C'est ce que prowrent les Diagnoses latines de Latham, de Gmelin, ef de .I. Temminck lui-mème, qui, toutes concordantes entre elles, sont inapplicables a la figrure que cet Ornithologriste a le premier donnée de son Kurukuru, Columba purpurata.

La diagnose de Latham porte :

Col. viridis, pileo coccinco, cristo fla'n; capite collo pec-toreque cinereo-albis. (Ind. ornith., p. 269, n 1\%.)

Celle de Gmelin :

Col. viridis, subtus in cincreum vergens, fronte et verticis parte purpareis, crisso flawn, remigilus nigris. (Limn.. $\mathrm{n}^{\circ}$ 64.)

Celle de M. Temminck:

Col. viridis, fronte purpurascenti, capite colloque cinereoalbis, cristo flavo, apice cande virescente; remigibus nigris : rostrum nigrum, apice albo; pedes nigri. (Hist. natur. des Pigeons, in-8, 1813, t. $\mathrm{I}^{\text {er }}$, p. 4.74.)

M. Temminck fait suive edte diannose de celle d'une variété, ou prétendue telle, conçue en ces termes:

Var. Fronte pilcoque minus saturatiore et in quibusdam vertex absque ullà rubedine, capite colloque cinereo- 
virescens, cristo aurantio; rostrum nigrum : pedes rubicundi ;

dont plus tard il fit une espéce distincte, ansı qu'on le verra bientôl.

C'est en effet au $\boldsymbol{K}$. purpuratus, le plus anciennement connu, que l'on a rapporté comme varicté tous les individus, si nombreux aux ìles Wharquises, dans les différents archipels de la mer du Sud ou de l'Océanie, et même dans la Nonvelle-Hollande, qui empruntaient quelque chose do leur coloration si brillante et si diversement nuancée au plumage de cette espèce, dont on en faisait alors comme le type.

Ainsi l'on voit dans Latliam, dont les premières publications de M. Temminck ne sont que la reproduction presque textuelle:

Var. Fronte pileorne mimis saturatis; quibusdam vertex absque rubedine.

Dans Gmelin :

Frons et vertex varius concolor, ant flave lined circumscriptus, caput collumque ex cinereo virescentia.

Il en est résulté qu'une fois les variétés de l'espèce admises a l'infini, chacun a pris pour type celle qui lui convenait le micux, sans s'oceuper de mette d'accord arec sa propre description délaillée la Diagnose Linnéenne qu'il en donnait.

Latham a pris pour type de l'espece et de sa description, conforme en cela à sa diagnose, la variété dont la calotte pourprée est privée d'un cercle jaune, et dont le ventre, à l'exception de la région anale, est vert; ne 
considérant que comme variété les individus d'Otahiti et de Tonga-Tabou dont la calotte pourprée est entourée de jaune.

Et voici comme il la décrit :

( Le bec est aux trois quarts d'un blanc jaunâtre; "l'iris d'un jaune pâle; le front est couromé de pour" pre; la tête, le con et tout le dessous du corps sont "d'un vert pâle tournant au cendré; les couvertures " inférieures de la quene ct la rigrion anale sont jaunes; n) le dessus du corps est d'un joli vert à reflets; les ré") miges sont noires, les deux externes extérieurement, ") les autres bordées de vert; les secondaires sont fran"gées extérieurement de jaune; la queuc a 3 pouces ") de longueur; les rectrices, d'un noir verdâtre, sont ") vertes extéricurement; lorsque la queuc est étendue " on voil une barre verdàtre vers la pointe, mais cefte n couleur, en l'examinant bien, n'occupe que la page " intérieure de chaque penne, en sorte que cette bande, " au lieu d'ètre continue, est réellement interrompue; ") les pieds sont d'un beau rouge ou d'un noir brunâtre; ") les ongles sont noirs. )

II. Temminch, an contraire, prend pour type et comme variété la plus constante, selon lui, les individus dont la calotte est encadrée de jaune, et il accompagne la figure qu'il en donne, planche XXXIV de ses Pigeons, de la description suivante :

"Nous figurons dans la planche XXXIV de l'édition ") en grand format, le Kiurukuru màle tel qu'on le trouve " habituellement. C'est dans cet état que nous avons eu " occasion d'en examiner plus de vingt individus ve- 
3) nant d'Otahiti; nous un atrons encore irouré deux au »Musémn de Paris, rapportés par les naturalistes qui b accompagnirent le capitaine Baudin dans son voyage » de découvertes aux terres australes.

") Cette première variété, qui nous paraît la plus n constante a tout le hau de la tète d'un heau rouge n) rose; cette couleur est entourée, dans les inclividus s adultes, paì une band jame. L'occiput, le cou et la » poitrine sont d'un gris cendré, nuancé de légères „) teintes verdâtres, plus sombres cependant sur la "partie posterieme da rou. Toutes les parties supé") rieures du corjes sonl d’un beau vert lustré marqué "de taches verte's, plus foncées sur les grandes couver» tures des ailes les plus proche's du corps; les conver") tures moyennes sont frangées de jaune, les rémiges ") sont noires intérienrement el rertes sur les barbes exn téricures; la deuxime penne de l'aile est terminée " en pointe. Les harbes exléricures de toutes les pennes " caudales sont rertes, mais les barbes intérieures sont " noirâtres; l'exlrémité de toutes les pemnes est d'un ") blanc nuancé de vert; le ventre a plusieurs nuances " de jaune ef d'orangé; l'abdomen el les couvertures ") inférieure's de la queue sont jaunes; les tarses sont à " moitié emplumés; le reste, ainsi que les doigts, sont ") couverts l'écailles noires; l'origine du bee est de " celte couleur, mais la pointe est blanchatre; les yeux s sont d'un jaune pâle. )

Ce qui résulte de ces deux descriptions, c'est qu'elles concernent réritablemeni deux individus diflérents, deux variétés distinctes; avant de décider si olles se 
rapportent à la même espèce, il s'agit de comparer chacune d'elles avec la diagnose la plus ancienue du C. purpurata.

Or il est évident, d'apris les cilations que nous en arons faites en commenesant, que la description de Latham est la plus exactement conforme à sa diagnose, qui, comme type, doit avoir la préférence el la priorité. Il n'est pas moins évident que M. Temmincli a reproduit textuellement la mène diagnose que Latham et, Gmelin, sans s'occuper de mettre d'accord arec elle la description qu'il a prétendu donner de la méme espèce, car sa diagnose et sa description sont en contradiction manifeste.

D'où la consequence que le type du $\boldsymbol{K}$. purpuratus est bien récllement, à l'heure qu'il est, la varićté qui a servi à la description de Latham, la description de M. Temminck concernant certainement une autre espèce, que nous croyons devoir se rapprocher de l'Ewingii de Gould, ou Roseicapilla de Lesson.

Ceci nous est même clairement démontré par la description que cet ornithologiste a faite d'une variété à laquelle il a cru plus tard pouvoir imposer le nom de Viridissima, et yue nous considérons comme identique, malgré l'absence de toute bordure jaunàtre aux rémiges et aux couvertures alaires, avec la variété typique décrite par Latham, dont elle serait, selon nous, la femelle, car tous les autres caractères de ptilose sont exactement les mêmes que ceux signalés par l'auteur anglais pour sa $C_{\text {. }}$ purpurata.

Quant à cette variété ou espèce prétendue C. viridis- 
sima, constatons que N. Temminck, qui avait recu son individu de Timor, a fait un rapprochement et une citation des plus inexacts en la considérant comme la même que celle que Latham dit être propre aux îles de Tonga-Tabou et d'Ulieta.

Voici en eflet comment il s'exprime (IIisı. des Pigeons, t. ex $^{\text {, in- }} 8^{\circ}, 1813$, p. 283) :

"Une variété que Latham dit être propre amx îles de "Tonga-Tabou ef d'ticta, mais qui se retroure aussi „) à Timor, diffère de la précédente (c'est-i-rlire le celle ") que Temminck venait de décrire conme type de son n Kurukuru, C. purpurata), en ce que le front et le " sinciput sont d'un violet pourpré tris-foncé, suns étre Dentouré d'une bande janne; le vort de toutes les parties ) supérieures est plus foncé (+t bleuàtre; les convertures "ne sont pas frangées de jaune; le ventre, ainsi que " l'abdomen, est vert; enfin les pieds sont d'un brun ") rougeâtre, el le bec est entiorement noir. Les natura"listes français qui firent partie de l'expédition com" mandée par le capitaine Baudin, ont trouvé cette der» nière variété du Kurukuru dans l'île de Timor. »

Et immédiatement il fait figurer cette variété comme jeune de son Kurukuru, dans la planche XXXY de ses Pigeons.

Puis plus tard, et en 1824, dans un article relatif a sa Columba diademata, pl. col. 20̈4, il en résume de nouveau en ces termes les caractires principaux, en élévant cette variété, ou jeune âge du Kurukuru pourpré, au rang d'espèce, sous la dénomination de C. viridissima : 
"Nous possélons les sujets de la Colombe viridissime " de Tonga, dont, les caractères les plus marquants ) sont : $1^{\circ}$ un plumage teinté généralement de diffé" rentes nuances vertes; $2^{\circ}$ point de couleur jaune ou ") orange au ventre, qui porte un ceinturon vert très„) foncé; $3^{\circ}$ point de bordures jaunes aux ailes, ni de „) jaune au menton; ef l. tont le plumage plus soyeux, „) et l'échancrure aux plumes de la poitrine à peine mar" quée. ")

Or Latham (Gen. Siyn., 1. II , 1783, 1. 626) se borne a dire :

"Les individus d'Otahiti ont la calotte d'un pourpre ") tres-laible; ceux d'Lliacta et des autres iles voisines "l'ont plus foncé; quant à ceux de Tonga-Tabou, ils ) ont le sommet fie la thete d'un pourpre excessivement ") vif et encadré de jaune; le bec est noiràtre; l'iris " jaune; la région anale orangée, et les pattes sont d'un ") rouge foncé. »)

Comme on le voit, il n'y a pas la moindre concordance entre le texte do l'auteur anglais et la citation de l'ornithologiste hollandais.

Quoi qu'il en soit, le rapprochement de la description donnée de cette variété par M. Temminck, de la description donnée par Latham de sa C. purpurata, suffit, ce nous semble, pour démontrer que les deux descriptions ont rapport à une seule et même espèce.

Une description du C. purpurata, qui est à reporter igalement à une autre espèce, est celle donnée par Lesson (Traité d'orn., p. 4\%2):

“Tête purpurine, bordée de jaune; ailes et dos 
") verts, ponctués de bleu; queue vert hleu, bordée de " jaune; gorge jaune; thoras gris jaune; coinlure jaune; ") milieu du ventre vineux; ventre orangé ; couvertures ») inférieures jaunes; (queue brune en-dessous، ))

Citer cette description, e'est réfuter sullisamment l'assimilation que cet auteur en a voulu fine au C. purpurata.

Un individu exactement conforme it la description donnée par Latham du C. purpurata, existe au Muséum d'histoire naturelle de Paris, ou il a étí rapporté par Quoy ef Gaimard de l'expédition de l'Astrolube. Il provient de Tonga-Tabou, et ne differe rle cette description que par une largge tache d'un vert noiràtre à reflets d'indigo cxistant au bas de l'estomac, ce qui le rapproche beaucoup sous ce rapport du Viridissima de Temminck.

Nous rappellerons en terminant qu'un caractère important que le Kurukuru pourpré partage avec quelques-uns de ses congéneres, est celui de la forme des ailes, déjà indiqué en ces termes par M. Temminck:

«Dans l'espèce du Kurukuru, les grandes pennes ») alaires vont en diminuant de longueur jusqu'd l'extí») rieure, qui est la plus courte de toutes: celle-ci est )) terminée en pointe.)

Ce qui donne it ces ailes une conliguration arrondie ou obtuse, tandis que d'autres espéces les ont d'une forme presque aiguë.

Notre but, en insistant sur res details à l'occasion de alte espece, qui derient pour nous non-seulement le yoe du senre huruluru. mais le type sprécifique du 
rai Kurukuru pourpre Columba purpurana), a ele de mettre les ornithologistes en garle contre la confusion introduite depuis longtemps par les autenrs dans ce groupe, et de bien les fixer sur les caractères de cette espèce typique.

Dans une note du voyage de l'Uranie, page 3', Quoy et Gaimard nous apprennent que cette espèce est triscommune dans l'ìle de Cuam; qu'ils en araient tué un grand nombre, qu'ils en ont même possédé "longtemps ") un couple mâle et femelle pris sur le nirl, dont les „) individus ne differaient pas le moins dı monde l'un ") de l'autre, tant pour lit couleur que pour la grosseur. w Les Mariannais, ajoutent-ils, la nomment Totot, et ") les Papous Manobo. Elle fait sa principale nourriture ") du fruit rougge d'une orange épineuse (Limonia trifo") liata) qu'elle transporte partout, ot contribue par ce ») moyen à multiplier d'une manière fort incommode. "

\section{KURUKURU POUKIOBOU.}

Kurukuru superbus, O. des Murs et Fl. Prévost.

Ptilonopus - J.-13. Gray.

Columba superba, Temm.

Spec. chan. K. Viridis, capite purpureo, cervice subrubicunda, collo cano, alce spurice cingulo carulece, maculis ovatis ex carulescente nigris in tectricibus alarum, abdomine et tectricibus sub caudi albis. - Rostrum nigrum; orbitce pedesque rubri (Temm., Hist. des Pig., t. Iex, Ind., p. 474).

C"est à M. Temminck que l'on doit la premiere description de cette helle espice de Colombe (IIist. des Pig., tome I, in-8, 1813, p. 277, Pig., pl. 33 ). 
Si l'on s'en rapportait à ce qu'en dit l'honorable Ornithologiste, cette Colombe, d'apres les caracteres de Ptilose sur lequel nous arons dabli notre genre Kurukuru, n'y devrait pas figurer.

Cet auteur, en effet, dans la comparaison qu'il fait du $C$. purpurata et du $C$. superba ( p. 281), et dans les caractères differentichs (qu'il indique, insiste particulièrement sur celui-ci :

"Toutes les plumes du Kurukuru, dit-il, ainsi que ") des variétés de ce Pigeon, sont échancrées du bout, "tundis que celles du Pouliobou, étant sans échancrure, " sont arrondies."

Nous signalons ici une erreur manifeste. Il suffit d'examiner attentivement ces plumes chez le $K$. superbus, pour se convaincre qu'elles sont également échancrées et bifides à leurs pointes : seulement les barbules de droite et de saluche, au lien d'ètre rigides et de finir en pointe des deux cotrés, sont molles conme toutes les autres et légerement arrondies. Le doute à cet égard n'est donc plus permis, car le fait est exact.

Quant aux rémiges, au lieu d'atre arrondies et ètagées, comme chez le K. purpuratus, elles ont une forme presque aiguë, les premières étant les plus Iongues, ainsi que le dit fort justement 11 . Temminck.

Nous ferons remarquer que dès sa publication son auteur n'entra dans les plus minutieux détails à son sujet que dans la crainte qu'elle ne fût considérée comme une simple variété de sexe ou d'àge du $k$. purpuratus. 
En voici la description, telle qu'il l'a domée pour le mâle adulte :

"Une calotte d'un violet éclatant couvre la tête; " l'occiput et les joues sont d'un vert tendre; la nuque n est colorée de brun rougeâtre; le manteau, le dos, " les grandes et les moyennes couvertures des ailes, ") sont d'un rert brillant nuancé de quelques teintes " olivacées; sur les grandes et sur quelques-unes des " moyennes couvertures se dessine, vers leur extré" mité, une tache ovoïde de couleur bleu noirâtre; n le poignet de l'aile porte une esprice d'épaulette d'un "bleu légèrement violacé; les pennes secondaires des " ailes sont noires sur les harbes intérieures, et d'un " vert foncé en dehors; toutes ces pennes, ainsi que " leurs couvertures, se trouvent bordées de jaune: " les rémiges sont noiràtres, hortées de jaune blan" chàtre; la queue, qui est composée de seize pennes, " est d'un vert olivacé à son origrine, d'un beau vert foncé " au centre, et terminée de blanc nuancé de vert; les " trois pennes laterales de chaque còté sont noires; les "autres ont leurs barbes intérieures de cette couleur; " le dessous de la queue est gris, et termine de hlanc. " Diverses nuances de gris cendré et de violet tendre " sont répandues sur le devant du cou; l'origine de $n$ toutes les plumes de cette partic est colorée de violet, " tandis que leur extrémité est grise. Ln large croissant. "d'un bleu foncé ceint la poitrine; le ventre et l'ab"domen sont blanes; les flanes sont verts, marqués de " taches blanches; les couvertures inférieures de la " queue, blanches depuis leur origine, ont sur leurs 


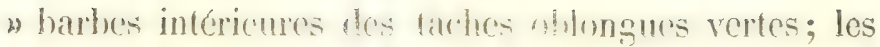
" yeux, placés dlans un cercle dénué de plumes, sont "rouges; le ber est couleur de comme, et les pieds sont ") rougeâtres.

"Longueur totale, 9 pouces $1 / 2$.

"Habit. Otahiti. "

Cette esporer, dont il n'existait qu'm soul individu lorsque II. Temmincle le dérrivil, ỵui chait encore in-

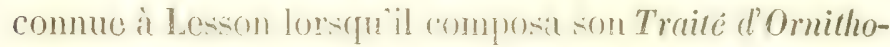
logie en 18.34, juisqu'il nn l'y menlionne que dans une note, page 4.72 , est devenue depuis assez commune pour être suffisamment connue et distincte da $K$. purpuratus, avec lequel il n'est plus possible de la confondre.

Pour ce qui aste l'individu decril dans le deuxième

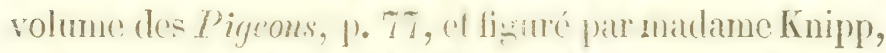
pl. 4.2, commet fomclle du K. suprobus, d'apres les rensoigmements mimes de. M. Temminck; nous croyons, après un mût examen, gue aet indivilu doit constituer une espèce a frart dont nous allons donner la derieription.

\section{KURUKURU DE TEMMINCK.}

Kurukuru 'Temminckii, O. des Mur's et Fl. Prévost.

Columba superba, famina, Fl. Prévost et Knipp., Pig., t. II, p. $77, \mathrm{pl} .42$.

Ptilonopus superbus, J.-B. Gray.

SPEC. CHAH. K. viridis; suprà aurate; lectricibus alarum caruleâ maculi notatis; gutture ex cincreo-grisescenti; pectore corulescente viridi; abdomine flavidè albo; laleribus viridi 
auratis; rectricibus viridibus, apice cinereis; rostrum cceru lescens, oculi rubri, pedes fuscè rubidi.

Voici comment sexprimait celui d'entro nous qui décrivit cette espexce conme femelle du $K$. superbus dians le deuxième volume des Pigeons :

"Nous avons ou un touble molif pour fire comnaitre ") la femelle de cette espèce : r'est d'aluned gu'elle ditlere ") beaucoup du mâle, et ensuilu, c'est flur malgré cotte

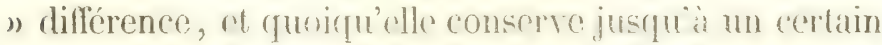
"point, par complarison avee celui-ci, l'un des plus " brillants, il est rrai, et dos plus richrment coloriés "des Oiscaux du gremre gui nous ocrupe, cette simpli") cité relative de roloration, raratipe habituel des " femelles, est elle-méme rncore aseer brillante pour " qu'il doive infailliblement arvines grion la considere "comme le mâle d'une autre espèce, si on la voyait "s seule et si l'on n'était point averti.

») Cette femelle n'était pas connue lorsque M. Tem) mincli a public la figrure of la description du mâle ) dans le premier volume de cel onvrage. Depuis il l'a ") reçue de plusicars localilés, of a bicn voulu nous » mettre à même de la figurer en nous en envoyant ») deux individus, l'mu flescribhes, l'autre de Ternate, n) dans les Molucpues, qu'il considire tous deux comme des "femclles, quoiqu'il cxiste cutre cux ruclipuesdifférences. » Ces différences tiennentwcllus à l’àge ou à la Jocalité, » ou bien l'un de ece indlividus ne serait-il pas un jeune, „) comme nous sommes disposé à le penser? C'est ce ) que, faute de renseignements, il nous est impos) sible d'examiner, et nous me pourons, thume c̀ présent, 
"quadmettre la détermination qui a élés élablie et 1) adoptée."

Nous avons en effet toujours douté, dès le principe, de cette identitid'esprece entro les deux individus. Lne étude plus attentive que nous avons faite depuis n'a cu pour résultat que de nous confirmer davantage dans nos doutes primitils. Co: qui nois determine anjourd'hui à cette spécification nouvelle et distincte, c'est l'observation (fue nous arons constamment faile, ofservation confirmée par tous les voyageurs, que dans le groupe de Pigeons composant le grand genre Ptilonopus de Swainson, jamais la femelle ne diflère autrement du mâle que par des teintes moins hrillantes dans les couleurs, qui sont presque toujours les mêmes chez les deux sexes.

C'est, au surplus, ce que M. Temminck lui-même a déjì recommu à l'oceasion ales $\mathbb{K}$. diadematus ou xamthogaster et purpuratus, dans le texte explicatif du n² 254 de ses planches coloriées, où il dit :

( La Colombe diadema et le Kurukuru forment en » effet deux espèces ou races distinctes dont nous ) connaissons maintemant les deux sexes, et que nous

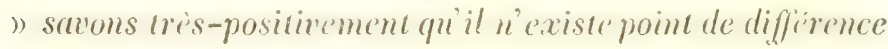
) marquée de livrée; les mâles, chez les deux espèces, " portent seulement des couleur's plus vives que les ) femelles. )

Nous regrettons bien certainement, pour asseoir notre conviction d'une manicre cucore plus solide, de n'avoir pas en nos mains les ciléments de comparaison que possède le célèbre Ornithologriste hollandais; mais, 
en l'absence de ces matiriaux, nous croyons que les deux exemplaires quiil a enroyés sont suffisants pour appuyer notre opinion.

En attendant, nous allons reproduire la description qui en accompagnait la figure.

"Celte espèce a le dos, le dessus des ailes et de la » quene d'un beau vert brillant à reflets dorés; sur le " milieu de la partie visible de chacune des plumes des ») couvertures des ailes, on distingue une tache, en ») forme de goutte, d'un bleu foncé, ce qui donne à ") cette partie un aspect tres-remarquable. La gorge est ") d'un gris cendré qui prent une trinte d'un vert hleuâtre „) en deseendint vers la poitrinc. Celle-ci est entierement " couverte, jusqu'à sa partie inférieure, de plumes » échancrées de cette méme couleur. Le ventre est d'un " blanc jaunatre, et les flanes sont du mème vert que le " dos. La quene est d'un vert foncé, et les taches qui en ») terminent les pennes sont grises à leur extrémité et "n'ont point de blanc. Les yeux sout rouges, le bec est ") d'un gris bleuitre et les pieds sont d'un rouge terne. ")

La longueur totale est de 10 pouces.

Ln caractire entre autres bien suffisant pour distinguer spécifiquement ce Kuruluru du Superbus, est la forme des plumes pectorales, dont l'échancrure prononcée attire l'attention au premier aspect, de même que chez te Purpuratus, le Tanthogaster et le DupetitThouarsii, etc., tandis que le chez Superbus il faut y regarder de tres-près pour bien saisir ce caractere, les barbules qui dépassent le rachis dans cette espèce s'arrondissant au lieu de finir en pointe. 


\section{KRURUKURU A DIADÉME.}

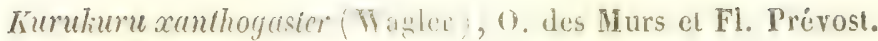
Columba xanthogastra, TWagl.

- - purpurata, Temm., pl. col.254.

- - diademata, Temm., pl. col. 254 , texte.

Ptilonopus flavigaster, Swains.

- - xanthogaster, Cr. R. Gray.

Sprc. char. K. suprì viridis, subtùs junquillaceus; capite, collo pectorefue fluriti: albis ; vertice à commissuri usque ad summum capitis lineâ flavâ circumcincto; scapularibus in medin cerulas; secundurias et ieyminibus faro marginatis; gutture flavo, lateribus cruribusque virescentibus.

On sait qu’à l'éporqu: vu M. Trmminck fit paraître le

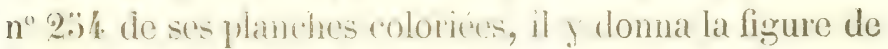
ce Kurukuru sous le nom de huruluru femelle (Columba purpuruta, lath.), tant on chait allors porté à référer à une seule et même espèce tout ce qui, de près ou de Inin, précentail le nuene ensemble de couleur ou de répartition de teintes.

Il fallut, pour relever presque immédiatement cette errour, qun () noy of Gitmard lissent de retour de leur si riche esploration de litranie, de s'occupassent fort heureusement de la publication des décomvertes de leur voyage. Ciest un restermus quils le firent (Foyage autour du monde de l'Uranie, p. 34, note 2) :

“Dans l'ouvrage qui fail suite aux Oiseaux de „) Buffon, par M. Temminck ( $43^{\mathrm{e}}$ liv., pl. 2ä4), ce

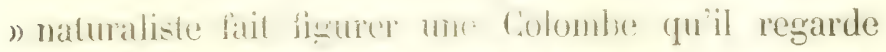
" comme la fimcile de l'esperce Kurulurti. Elle a le "dessus de la kive rembere, aver une bande jaune au- 
n dessus de l'aril. Si c'est me femelle, nous ne pensons " pas qu'elle doive appartenir à cette espèce. Ces Oi) seaux sont très-communs dans l'île de Guam; nous 》) en avons tué un très-grand nombre; nous avons

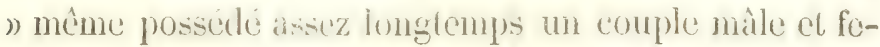
n) melle, pris sin li. nid, dont les individus ne diffés raient pas le moins du monde l'un de l'atutre, tani " pour la couleur que pour la grosseur, et tous ces

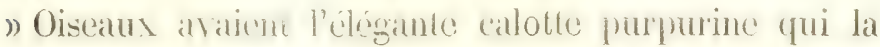
m) distingue. Voilit bien certainement ce rui existe dans n) celle île; pent-cire? ailleurs la lenclle prósente-t-elle ") quelque différence. »

C'est alors yue .r. Temminck s'empressia de faire la rectification suivante :

« Induit en erreur par des données inexactes, nous n avons publié le portrait de cette nourelle esprice sous ") le nom de Rruruliuru femelle (Columba purpurata, „ Lath.); l'enscmble des formes firait en eflet présumer n) que notre Oisuan, figuré planche 20.5, est la femelle "de cette "speco. Des renseignements ohtenus depuis, ") ainsi que l'examen de plusicurs sujuts des deux sexes, n et le doute émis fral MII. ()uoy el Gramard, an sujet n) de celte identité jrisumé(e, nous mettent à mène de D) revenir sur cet article, w yue la Colombe diadème et ") le Kurulum forment en effet deux especes on races \# distinctes, dont nous comnaissons maintenant les deux ) sexes, et que nous savons très-positivement qu'il "n'existe point de différence marquée de livrée, les ") mailes, chez les doux especes, portant seulement des "teintes plus vives (que les femelles.") 
En voici maintenant la description:

Dessus de la tête, depuis la commissure du bec jusqu'au sommet du vertes, d'un blanc mat d'un aspect cendré, encadre par une lig̣ne jaune-serin partant de la commissure, passant en forme de sourcil au-dessus de l'oil, et allant en s'élarwissant au vertex. Le menton et la gorge sont du même jaune-serin. Nucque, côtés de la tête et clu cou, et poitrine, d'un blanc légèrement jamnatre : cet aspect jaunatre al la poitrine tient à la forme échancree des plumes de cette partie, dont la base élant jamitre sert comme de fond à la conleur blanche des barbules idhancreses, qui viemment s'y détacher d'une manière assez tranchée. Dos et manteau verts: cette couleur est aussi celle des petites couvertures des ailes; moyennes courertures vertes largement bordées de jaune; wrandes couvertures d'un bleu foncé rgalement bordies de jaune; remigges primaires d'un vert blenâtre foncé sur leur page externe, qui est finement lisérée de blanchâtre, vertes sur leur page interne; ventre, dans toute son étendue, d'un beau jaune jonquille; flanes et tarses d'un rert nuancé; queue d'un vert foncé, terminée par une large bande d'un vert clair variant du grisâtre au blanchâtre; yeux rouges; bec blanc à la pointe; doigts noirs.

Iongueur totale de ...... 20 à 21 centimètres.

Irabir. L'île Banda. 


\section{KUKUKURU DUPETIT-THOUARS.}

Columba Dupetit-Thouarsii (Néboux), Rev. zool., 1840, p. 289; Voy. au pôle Sud, pl. 29, fig. 1.

Ptilonopus leucocephalus (Gray), Birds. Brit.mus., 111, p. 2. Columba kurukuru purpureo-leucocephalus (Hombr. et Jacq.), Ann. sc. nalur., is41.

\section{Ptilonopus ——____ (G. R. Gray).}

- Emilia (Lesson), Ech. mond. sav., 1845, p. 871. Kurukuru Dupetit-Thouersii (Néboux), Fl. Prévost et O.des Hurs.

Cаar. spec. Plil. suprà viridi-olivaceus; capite albo, stria aurantiaca cincto; gutture flavo; collo et loris cinereo-virescentibus; pectore medio aurantiaco; abdomine et crisso pallide pavis; remigibus primariis in prima dimidia parle à stipite nigris, in ultima, ad apicem intensè viridibus, albo favido striclè, secundariis eodem viride latius flavo, marginatis; tegminibus majoribus viride splendidè ccerulescenti notatis; rectricibus lati metallicique viridibus, ftarn pallescentibus ad apicem.

Description. Le docteur Néboux a donné de cet Oiseau, dans la Revue zonlogique de 1840, parge 289, la description succincte suivante:

Hâle adulte. "Calotte d'un blanc pur entourée d'un „) liséré orange; dos rert olive: vontro d'un beau jaune n orange; région anale jaune serin; ailes : première " penne très-rétrécie dans son tiers postérieur, la qua") trième est la plus longue; rémiges secondaires et cou》) vertures supérieures à reflet vert doré métallique, s bordées de jaune et présentant une tache bleue au „ centre; queue légèrement fourchne; rectrices d'une "rouleur verte à reflet métallique, puis d'un blanc jau. Orseatix. 
) nâtre à leur's extrómités; tarses rouge orangé; ongles "noirs; bec noir à la base, blane à l'extrémité; iris ) rouge. )

Habit. L'île Cihristine, l'une des Marr[uises.

Les indigènes l'appellent Kíouliou.

Nous allons compléler ces caractères principaux par les détails de ptilose qui suivent :

Tout le dessus de la tète, depuis les narines, d'un blane mat entouré d'un cercle étroit de couleur orange; gorge jaume pâle; con et lorums d'un condré verdâtre mat; tout le dessus du corps d'un rert légèrement oliVâtre; rémiges primaires noires dans leur moitié interne, d'un vert foncé dans le surplus, fincment lisérées cxtérieurement d'un filet blane jaunàtre; rémiges secondaires également vertes, lisérées plus largement d'un jaune serin; les grandes courerfures présentent vers leur pointe me large tache angulaire d'un vert fournant au plus beau bleu foncé; quelques traces de cette dernière conleur se retrouvent clairsemées sur les pelites convertures; milieu de l'estonac jamne orange, tournant au curmin violacé au centre et quelquefois au minium, d'après un bel individu rapporté au Muséum d'histoire naturelle de Paris par M. Mercier, attaché au jardin bolanicue; abdomen et comvertures inférieures de la queue jaune serin; rectrices d'un bear vert dans les deux premicrs tiers de leur longueur, à reflet métallique jaune pâle dans le surplus jusqu'à la pointe,

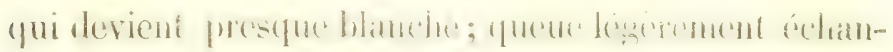
crée; la première rémige, un peu plus courle que la

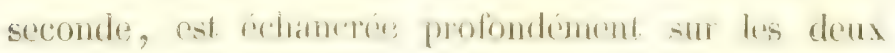


bords et réduite à une espèce de ruban, ce qui est un des caractères des vrais Ptilonopes.

Longueur totale....... 25 1/2 centimètres.

C'est le même Oiseau qui, depuis la publication que le docteur Néboux en a faite dans la Revue zoologique de 1840, a été décrit par M. J.-E. Gray (dans Birds in the Brit. Mus., III, 1). 2), sons le nom de Ptilonopus leucocephalus, et par Lesson (Écho du monde scwant, 184.3, p. S\% 1) sous celui de Ptilonopus Emilice, réformé dans ses Suites à Buffon, 184\%, page 209.

C'est encore le même Oiseau que MLI. Hombron el

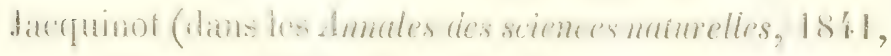
zoologie, p. 316) ont décrit de la manière suivante, et sous une dénomination si cn dehors des règles linnéennes:

«Colombe kurulieuru lencocéphale.

»Columba tiuruliuru purpuro-leucocephalis.

„ Tariété de la Colombe kurukuru femelle. Cette Co" lombe leucocéphale (à tête blanche) est une jolie va-

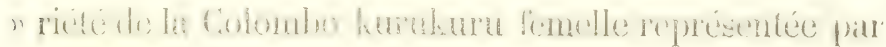
") Temminck"

》) Tête blanche, entourće d'une auréole jaune d'or; » cou vert grisùtre; dos, tectrices, vert à reflets jaun) nâtres; de chaque côté trois tectrices scapulaires ma») culées dans leur centre de bleu azur, cntouré de vert ") semblable à la tcinte générale du dos; rémiges vert

1 'Temminck n'a jamais donné la figure de la femelle du Kurukun, mais ce qu il on considere senlement romue ame simple variété qui se voit à sa pl. 55. 
"vif, taché de bleu azur, lequel est bordé de vert ja "natre en arriere et de jaune en avant: queste verte at "reflet blen, traversie d'une bande jaune a som extrés milé.

Menton, gorge, jaunes; joues gris verdàtre; cou net plastron grivelés de jaune verdâtre et de gris ver" dâtre; poitrine et ventre roux rose; croupion et tec" trices caudales jaunes; cuisses jaune verdâtre; llancs " verdatres; tectrices alaires inférieures grrises, salies " çà et là de jaune verdâtre; rémiges gris ardoise.

"Pieds roses; bec noir, blanc à son extrémité antén rieure; iris jame.

"Taille $33^{4}$ centimètres. Habit. Nuhiva.")

C'est enfin lo mème Oiscau que ces voyagents, mieux renseignés, ont liguré dans la zoologie de leur Voyage au poile Sud, ()is., pl. 29, fig. 1, sous son veritable nom de Colombe Dupelit-Thouars. Il est probable qu'ils reviendront sur cette erreur lorsquils publieront le texte à l'appui de leur riche atlas.

Ces quatre descriptions, toutes concordantes, font suflisamment connaitre le plumage du màle adulte de cette espèce.

Les caracteres d'organisation et de coloration sont, on le voil, tous ceux assignés au genre Ptilonopus, si judicieusement créé par swainson : éest la mone forme de bec, de quene ef de farses logirement emplumes a leur naissance; c’est de plus la mème distribution gé-

' C'est 23 qu'il faut live; c'est certainement une erceur du typographe. 
nérale de conleur si remarquable, surtout par cette plaque occipitale presque toujours eacadrece et comne isolce du reste de la coloration; c'est enfin la mème nature de plumes à la rérion pectorale, toutes élant tronquées bifides et d'un aspect rigide.

Nous ajouterons que notre Colombe compose, avec le Pt. purpuratus (Swains.), Pt. taïtensis, Lesson (G.-R. Gray), Pt. xumhogaster (G.-R. Gray) ou diademau 'Temm.), un petit wroupe offrant les rapports d'ensemble les plus complets, et remarquable avant tout par la forme si exceptionnellenent acuminée des plumes fle la poitrine. Nous ne pouvions tone hésiter à classer notre espèce dans le nouveau genre Kurukuru.

Jaintenant on conviendra qu'il est difficile de ne pas apercevoir les rapports les plus intimes entre cette Colombe et la Colombe diadena de Temminck, Ptilonopus xanthoyaster (G.-R. Giay), pl. col. 234, rapports tels que ce n'est qu'après un long of minutieux examen qur: unom nous sommes decidris à an faire une espece distincte, que le docteur Néboux à dédiée à M. l'amiral Dupetit-Thouars, commandant de l'expedition.

Ainsi, ce que le Pt. xanthogaster a de plus remarquable, parmi ses congénères à plaque occipitale si vivement colorce, c'est d'avoir la sienne d'un blane mat pur, et d'aulant mieux indiquée qu'elle se trouve circonscrite par une bande étroite, d'un jaune serin, partant des narines, passant au-dessus de l'œil, en forme de sourcil, et allant, en selargissant, contourner cette plapgue banche pardessur la nugue, nir elle forme vraiment diadème. 
Or cette plaque est la même dans notre espèce; elle

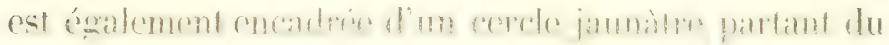
même point et décrivant la même ligne que dans le Pt. xanthogaster : seulement ce cercle est dans tout son parcour's très-étroit, d'un jaune faiblement orangé, moins apparent néanmoins que dans cette dernière, le fond de coulcur sur lequel it se dessine chez clle étant d'un blane prescue pur, tandis que, chez la nôtre, ce fond est d'un cendré verdître mat et terme, presque comme chez les Trerons; la gorge estégalement jaune, mais d'un jaune soufre; enfin les taches blenes des grandes couvertures et leur liséré jaune sont les mêmes.

Mais ce qui l'en distingue complétement, à part la coulcur cendrée du cou et de la poitrine, c'est d'abord une tache du mòme blane que le dessus de la tête, en forme de moustache, placée au-dessous de la mandibule inférieure, et encadrant ainsi à sa base la tache gulaire jaune; ensuite le ton olivitre de tout le dessus du corps, qui est d'un vert gai chez l'autre; puis l'absence de cette teinte jaune vil uniforme qui colore tout le ventre et les flancs du $P$ \% xanthogaster, cette teinte ćtant remplacée chez notre Oiscau par un jaune sale un peu verditre, et au milieu du ventre par une large tache rotige olangé passint au carmin violacé dans le centre; cnsuile une bande blanc jaunatro de près de a centimètres de largeur, qui tomine chacune des rectrices à la pointe, au lieu que celte partio ne so distingue, chez le $\ell$. xamthorgaster, que, par ine teinte d'un vert plus clair que le reste de la queue; of enfin la couleur des farses, d'un gris noiratre chez celle-ci, d'un rouge 
manoce chez collo-lit, salns parler do la taille, plus grande chez notre Oiseau de 3 centimètres au moins.

\section{KURUKURU DE SAMOA.}

Kurukuru Samoensis, 0. des Murs et Fl. Prévost.

Ptilonopus ——— J. Verreaux, mss.

Ptilonope de Marie, IIombr. et Jacr., Voy. au póle Sud, atlas,

Colombe de Vincendon.
rolumbe liuruliuru superbu. limmina. Ann. sc. nat.,
t. XVI, 1841, p.516,

$$
\text { Ois., pl. } 29 \text {, fig. } 2 .
$$

SPEC, cran. K. flavus; fronte, vertice, scapulo humerali et tectricibus subcaudalibus ex roseo vinaceis, pileo flavo circumcincto; reliquo capitis et collo albis levissimè flavidis; pectore rosaceo, albo striolato; collo post-infero rufo; primariis viridisplendentibus; secundariis, tcctricibus majoribus et rectricibus ex cinereo albis; illis fascid apicali viridibus; rostrum, orbile pedesque cerulci.

La première description et la première figure de cette

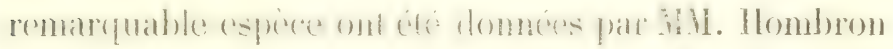
et Jacquinot, chirurgicns de la Zélée. Mais quoique récemment découverte, cette espèce est une de celles dont l'élucidation nous parait avoir le plus d'importance, par suite de la confusion qui règne dans sa dénomination scientilinge, ronfusion gure le tomps ne ferait qu'accroître.

Linsi dins les Ammles des scieners nuturelles, se sivir, 1. XVI, 184, P. 316, on lit l'article suivant, communiqué à l'Académie des sciences rle Paris par ces deux voyageurs :

"Colombe Kuruknu de Vincendon (Columba liuru"kuru superba), focmina. 
" En dessus, tète pourpre, tendant un peu vers le b) roux, entourée d'un cercle jaune peu marqué; cou „) blanc, traversé d'une bande jaune d'or à sa base; ) cointuron pourpre, étendu d'une épaule à l'autre; »dos, tectrices alaires et caudales, rectrices grises; " la circonférence des plumes seulement encadrée de „) jaune d'or un peu verdàtre; mais, au milien du dos ») et sur les tectrices caudales supérieures, la totalité de " la surface des phumes revit rette teinte brillante d'or m) nuance legrement de verdatre. Les rémiges sont vert „) éclatant aussi varié d'éclat que l'incidence de la lu"miere ot rariablu: rharume d'elles somt denticulées ") en avant de jaume pailie. Ine hande vert brillant tran) verse la queue vers son extrémilé libre.

„En-dessous : menton, gorge, cou, blanes; plumes b du plastron grivelées de rose et de blanc : ce joli ") plastron se détache sur un fond orangé, qui orne le

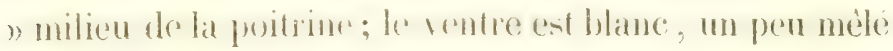
nde jaunâtre; les tectrices caudales inférieures sont " presque roses; les pennes sont grises.

„) Iris blanchâtre; pieds et bec gris verdâtre.

n) Taille 33 centimètres (c'est 23 qu'il faut lire).

"Habit. Les iles Viti. Notre spécimen provient de ") Balaan. ")

Et d'abord, pourquoi lui domer en françats un nom spécifique nouveau, alors qu'on recomnait dans la mème ligne que l'individu pourtait n'ètre qu'une femelle du C. superba, et qu'on ajoute entre parenthèses Columba kurukuru superba, famina.

rette contradiction valait au moins la peine d une ex- 
plication dans le cours de la note, ef on ne l'y trouve pas.

Ce n’est pas tout : l'exemplaire ainsi décrit est figuré par les memes voyagenrs dans l'atlas de leur foygage an póle Sud, pl. 29 des Ois., lig. 2; on va sans doute lui rlonner au bas de la planche l'un des deux noms insérés dans la note lue à l'Académie des sciences? Nullement. C'est un troisième nom qu'on lui donne, et ce nom est Ptilonope de Marie.

Et pas un mot d'observation pour sider à débrouiller re chaos!

En présence d'une confusion semblable, qu'il ne nous était pas possible de ne point signaler, nous n'avons vu d'autre moyen d'en sortir, quant à la dénomination scientifique it domer a reftrepres masnificque ef encore bien rare, que de lui imposer celui de Samoensis, que nous avons trouvé dans les noles manuscrites si précieuses de J. Verreaux, qai a rapporté au Ituséun d'histoire naturelle de Paris deux exemplaires de la mème espèce, dont un parfaitement adulte, qu'il a eu vivant, et d'une fraticheur de plumage extraordinaire.

Voici la description fort exacte qu'il en a faite dans ces notes, et que nous reproduisons textuellement :

"Calotte d'un beau rose vineux très-brillant, légèren) ment bordée de jaune pâle; tout le reste de la tête et "du con d'un blanc mat légerement teini de jaune, "c'est-à-dire que le centre de chacune des phumes est ") de celle dernière couleur; une tache d'un jaune rous") sitre sur le bas du derrière du cou, suivie d'une large "hande transversale du mème mäe vineux que sur le 
") sommet de la tête, mais cette bande s'étend jusque „) sur les petites couvertures des ailes; le reste des par» ties supérieures est d'un jaune olivâtre qui devient. "plus foncé sur le croupion et les couvertures supé) rieures de la quene : cette mème couleur est aussi rés paudue sur les ailes et s'y trouve mélangée de gris; ) toutes les rémiges sont d'un vert britlant, excepté les "plus rapprochées du corps, qui sont grises, frangées " de jaune olivàtre. " (Toutes les rectrices sont grises et portent à leur extrémité une large bande du mème vert que celui des rémiges: colte bande n'existe que sur le côté externe des six rectrices latérales, et à I centimètre de leur pointe; les médianes n'en portent pas l'apparence et sont entierement d'un gris pàle ou blanchatre.) «Sur le devant du cou se trouvent des plumes " ćchancrées vers leurs pointes, d'un rose le plus beau nsur une partic de leur longueur, mais blanchàtres ") vers leurs pointes. ) (En sorte que le cou et la poitrinc paraissent grirclés de rose et de blanc.) "Au" dossus de ces plumes se trouve une tache roussàtre, n) mais en relevant ces plumes il est facile de voir le ") rose qui en colore le centre: tout le reste des partics " inférieures est d'un jaune pâle, excepté les couver") tures inférieures, qui sont encore du même rose vin) neux que la tête. L'œil se trouve entouré d'une cou" leur bleuâtre, et colle dernière couleur existe sur les "pattes et la moitié du bec, dont l'extrémité est oli") Vâtre; celui-ci est trìs-court et légèerement vouté; les ") orgles sont bruns; l'iris est blanc cerclé de rouge )) pâle. 


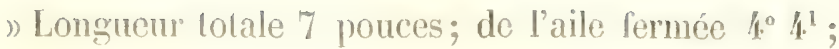
) de la queue $2003{ }^{\circ}$.

„La première rémige se trouve échancrée et exces》) sivement rétrécie vers son extrémité d'environ $1^{\circ}$. »

Les tarses sont emplumés dans les trois quarts de leur longueur.

Trouvée à l'ille Samoa, l'une des Navigateurs.

\section{KURUKURU DE TAITI.}

Kurukuru Tailensis, O. des Murs et Fl. Prérost.

Pilonopus G. R. Gray.

Columba kumliuru, var. Taïtensis (Lesson), Voy. de la Coquille, $1^{\mathrm{er}}$ rol., 1.29\%.

Chali. spec. Ptil. fronte ac pileo pallidè roseis, lined flavâ circumscriptis; collo, corporeque inferiori cinereis, viridi pallide tinctis; gula pectoreque pallescentibus; corpore superiori viridi-aurato ; cauda apice albido fasciald.

Les ornithologistes sont généralement d'accord aujourl'hui pour considérer comme espèce distincte du Ptilonopus purpuratus (Sivains.) ou Kurukuru, celle décrite par Lesson comme Columba liuruliuru, var. Taïtentis, et dont il a donné la description suivante dans le Voyage de la Coquille, 1. 1 ${ }^{\mathrm{er}}$, 1). 297:

"La Kuruluru d'Otahiti, dit-il, a la tailte un pen plus "forte que la varićté dle Timor dont elle se rapproches " le plus. La calotte purpurine qui revêt le sommet de " la tête est d'un rose très-pâle, que circonscrit une raie " asse\% large d'm jame pen intense. Le cou en entier, "jusqu'aux épaules, et tout le dessous du corps sont 
"d'un gris cendre miforme, teinte de vertatre plus " foncé sur la poitrine. Le menton, la gorge et le devant " du cou sont blanchâtres. La région anale et les coun vertures inférieures de la queue sont d'un jaune vif. "Le manteau, le dos, le croupion ef les ailes sont d'un ") vert doré avec des teintes rousses. Les rémiges sont "brunes en dedaus. La queue est régulièrement recti") ligne; chaque rectrice est d'un vert métallique en "dehors, brune en dedans ef termince par une large "raie blanchàtre bordé de brunàtre. Le here est plombe net blane a l'extrémite : il est recouvert dans l'état de " vie par deux petiles caroncules orangées qui surmonn) tent les narines. J.es larses a moitié emplumés sont " de couleur orangéc. "

C'est avec juste raison que le docteur Néboux a décrit sous le nom de Colombe de Träti, dans la Rev. zool., octobre 1840, p. 289, un individu qu'il ne considérait que comme une variété de celui décrit par Lesson.

"Celleque nous avons rapportée, dit-il, diflëre sur" tout de celle de M. Lesson en ce que la calotte est „violette, les tarses noirs, et en ce que la zone blan" châtre qui se remarque à la quene n'occupe pas prén cisément l'extrémité les rectrices: dans notre indi"vidu, cette zone se trouve à un centimètre de l'ex"trémité. "

Un individu exactement semblable à ce dernier avait

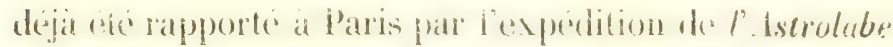
en 1829: il provenail de Tonga-Tabou.

Longuen intale.......221/2 centimitres. 


\section{KURUKURU DE NÉBOUX.}

Kurukuru Nebonxii (Fl. Préxost et O. des Murs).

Char. SPec. Pl. capite, collo, corporeque inferioricinereo leviter favo-virescenti; naribus et guld albidis; fronte verliceque cinereo-lilaceis; pallio et alarum tegminibus are virescentibus; majoribus primariisque latè ct melallicè viridibus; his latè, illis strictè flavo pallidè marginatis; crisso flavo; rectricibus apice externo flavo pallidè fimbriatis, interno cinereo fascialis. Caudâ furcald; rostro albo ; pedibus ac digilis nigris.

Nalqré les rapports asse\% intimes qui paraissont au premier aspect exister entre ceflu Colomine el le Pt. Trätensis de Lesion, nous n’avons pas hésite i la comsiderer comme comstituant une espere nomvelle parmi les Ptilonopes.

En voicila description : Tout ledesus de la trite à partir de la naissance du bec jusruiau sommet de la nuque est d'un condre lilas tres-clair ; la gorge el les narines sont d'un blanc fort legerement lavede jaunàtre, les côtés de la tête et du cou, le derrière de celui-ci jusqu'aux épaules, et tout le dessous du corps sont d'une teinte grise lewerement zonó du vort jaunatre; les plumes de l'estomac ont la forme lanceolée qui se remarque chez. les Pt. purpuratus, Taïrensis et Dupetit-Thouarsii, et se dessinent en gris sur le fond plus ou moins verdâtre ou jaundre que forme la premiere moilie de chacune de. ces plumes de cetle derniere corteur; le dos, les petites et les moyennes couvertures supérieures des ailes de même que celles de la queue sont d'un vert uniforme, légèrement olivâtres à reflets de hronze; les grandes 
couvertures, de même couleur, sont bordées extéricurement de jaune, les rémiges secondaires et primaires, d'un beau vert foncé à retlets métalliques, sont également bordées, mais très-finement, sur leur tranche cxtérieure, d'un jame blanchâtre, et sont noires sur leur page interne; la région anale est jaune jonquille; les rectrices, du même vert que les rémiges primaires, ont le reflet bronzé des plumes du dos, et elles portent toutes, à l'exception des deux médianes, sur la partic de leur tranche externe la plus rapprochée de la pointe, un fin liséré de jaune de 1 centimètre d'étendue, et dans toute la largeur de leur page interne, à partir du rachis, une bande gris cendré qui ne s'apercoit que lorsque la queue est étenclue. Le bec est d'un blane d'argent; les tarses et les ongles sont noirs.

Longueur totale......... 24 centimètres.

- de la queue....... 7 -

Elle vient de Taïti, d'où elle a été rapportée par

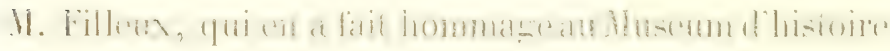
naturelle de Paris.

Sams doute la forme des plumes de la poitrine, l'ensemble semi-grisâtre et verdâtre de la coloration, cette teinte rosacée qui apparaît au sommet de la tểe, pourraient donner l'idée que cet individu ne serait peut-ètre bien qu’un jeune ou une variété du Pt. Tütensis, décrit par Lesson.

Mais tout doute à cet égard doit tomber derant les diflérences caractéristicnos qui séparent notre exemplaire de ce dernier; voici en eflet sur quoi reposent res differences oremingurs. qui sulliratent is la rigureur 
non-seulement pour la faire siparrer speritiquement de

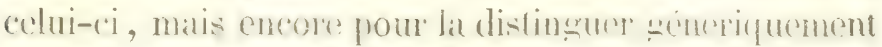
des vrais Ptilinopes.

La partie cornée du bec, d'une couleur blanchâtre, est plus renflée; les tarses sont emplumés à peine audessous du genou; la première rémige, de très-peu de chose plus courte que la deuxième (d'un demi-centimètre), ne porte pas d'échancrure bien sensible, et offre presque la forme habituclle des autres; les ailes sont, du reste, proportionnellement plus longues que dans les Kurulisurus : chez ces derniers, elles arrivent à 4 centimètres $1 / 2$ de l'extrémité de la queue; chez notre Colombe elles s'en rapprochent de 2 centimètres seulement; par la même raison, les srandes couvertures qui, chez les Kurukurus, ne sont éloignées, d̀ leur pointe, de l'extrémité des rémiges primaires que de 2 centimètres $1 / 2$, le sont ici de 4 centimètres; enfin, la queuc est positivement échanchrée, les deux rectrices latérales élant les plus allongées, et les deux médianes les plus courtes, mème pliée, c'est-à-dire à l'état de repos : ce qui établit entre elles une diflérence sans doute peu sensible à la me-

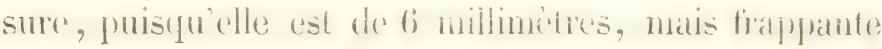
pour l'œil le moins exercé, qui ne saurait s’y tromper.

Nous avons retrouvé dans la riche collection du Muséum de Paris un individu exartement semblath an nòtre provenant également de Taïti, et qui en avait été rapporté par l'Expédition de la Zélée.

Nota. C'est par erreur typographique que notre 
planche VII, qui contient la tigure du Kuruhuru Vebouxai, porte le nom de Columba Dupetit-Thouarsii.

\section{KURUKURU DE SWAINSON.}

Kinrukuru Swainsonii, O des Murs et Fl. Prévost.

Ptilonopus purpuratus, var. Regind, Swains., Zool.j. I, p. 473, 1825.

Columba purpurata, Temm, pl. col. 254; Jard, et Selby, Ill. orn., vol. II, pl. $=0$.

Ptilonopus swainsonii, Gould., $P r . \approx . S, 1842, \mathrm{p} .18$.

Nec Columba roseicopilla, Iesson, Trait. d'orn. p. 4\%?

Spec. char. K. fronte et vertice splendide, coccineis, hoe colore linea angustâ flava nisi apud frontem cincto; pectore sordidè viridi, singulis plumis ad apicem bifidis, more furcula, cujus apices maculà triangulari argenter-cinered notantur; abdomine medio aurantiaco (cum maculâ media latè violaceâ); caudo apicè laté flavissimo (Gould, loc. cit.).

Cette espèce vient à l'appui de ce que nous avons déjà dit de la confusion a laquelleont dommelueu les rapports d'ensemble existant entre les différentes espèces de

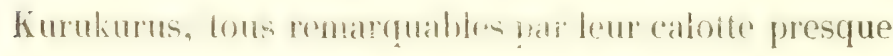
toujours purpurine, ronfusion qui a fait pendant longtemps rapporter à celte derniere tous les individus reconnus depuis ponr espèces bien distinctes. Ainsi, il a fallu près de vingt ans pour amiver à la véritable spécilication du Swainsonii. Temminck, à la mème époque, la considérait comme C. purpumu, dans la note rectificative de la description de son Dindema (xamthogoster), pl. col. 264, ou il s'exprime ainsi :

"Cette race constante, qui a pour patrie les îles Cé-

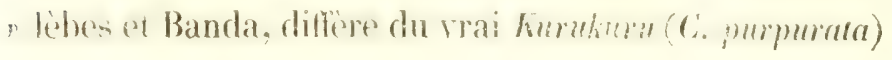


") de la Nouvelle-Hollande, et qu'on retronve anssi à ") Timor et à Guam. »

Lorsque Swainson fit connaître cotle espèce (Zool. Journal, vol. 1, p. 4.73, 1824-25), il ne la décrivit que comme une belle variété de $C$. purpurata, à laquelle il donna le nom distinctif de Rergina, aree la diagnnose suivante:

Pt. viridis; vertice purpureo roseo margine semi-lunari aureo; fascia lati abdominali aurantiaci; tergminibus inferioribus flaris; pemis colli rigulis apice furculis." Quelques ammées après, MII. Jardine et Selloy, restant dans les mèmes errements, en donnèrent lir ligure et la description, sous le nom de C. purpurata (Ill. orn., vol. 2, pl. 70 ), avec linquelle ils l'identifierent completement, confondant avec elle les prétendues varietios des Célèbes, d'Uliéta of de Tongi-Tabou, citées par Latham, et même celle de Timor.

Quant a la Colombe des Nariannes, decrite par Jesson (Traite d'ornit., p. 472) sous le nom de Columbr roseicapilla, nous croyons que c'est à tort qu'il l'a rapporter a la var. Regina de Swainson, dont elle nous parait fout à fait distincte, ainsi que nous l'expliquerons tout à l'heure, surtout par la forme arrondie de ses: atles: elle, se rapprocherait plutot du P’. Ewingii, Gould, dont nous parlerons bientôt.

Foici la deseription exacte du $K$. Suctinsomii, d'apris un bel individu du Naséum.

Front et occiput, à partir des narines, d'un violet carminé pur encadré, à partir de la dernière moitié du sourcil, par une fine raie on borlure d'un jaume serin 
vif; une lache bianche, partant de la commissure du bec, occupant le lor'um et s'amincissant à la naissance du sourcil, complite sur le devant l'encadrement de cette plaque violette; nuque et derrière du cou d'un

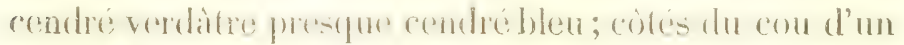
spis de perle: mentun ret gorge biancs so fomlant sur les bords dans le gris rles côtés du cou; toutes les plumes garnissant l'estomac et la poitrine sont vertes dans leur première moitié à partir de la base, et grris perle dans l'autre moitić vers la pointe : ce gris perle, d'autant plus remarquable et se détachant l'autant mieux sur le fond verdittre, que chacune de ces plumes est matte et sans reflets, et porte cette échancrure si caractéristique dans la ptilose de cette partie chez le $K$. purpuratus, et dont M. 'Temminck a donné la structure en clétail : dos, manteau, scapulaires et croupion d'un beau vert; petites couvertures des ailes d'un vert bleuâtre finement bordées de jaunàtre; cette bordure un peu plus large aux moyennes couvertures qui sont de même couleur' grandes couvertures de même couleur, se terminant par un large miroir blen, encadré largement de vert et le tout frangé à la pointe d'une bande jaune beaucoup plus

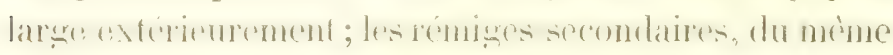
vert que les autres plumes, sont finement entourées de jaunâtre; il en est de même des rémiges primaires; mais celles-ci terminent en noir le còté externe de leur pointe qui est verte, ot ont leur page interne de même couleur; abdomen d'une belle teinte orange foncée; le centre de celte région portant à sa partie supérieure une laree ladhe du mème viodet carminé que la 
plaque occipitale; cette couleur orange se dergradant en jaume serin vers le bas de l'abdomen; plumes des culisses et des tarses vertes, les premieres largenent terminées de jaune scrin ; region anale ef couvertures inferieures de la queue du mène ton orangé que l'abtomen; rectrices vertes dans leur page exlerne, d'un noir cendre' dans leur page intrene : ces ileus coulenrs séparées vers le bas par une nuance blene, semblable à celle des moyennes couvertures's, el lerminées à la pointe par une large bande d'un beau jaune serin; couvertures supérieures de la queue finement terminces de jaunatre.

Longueur totale...... 24 centimètres $1 / 2$.

Cette espece parait ne se trouver qu'en Australie, ou elle habiterait exclusivenent, principalement daus la Nouvelle-Galles du Sud ; c'est de la Ririere-Clarence et de Morton-Bay que M. Gould l'a presque toujours recue.

\section{KURUKURU DES MARIANNES.}

Kurukuru roseicapilla, O. des Murs et Fl. Prévost.

Columba roseicapilla, Lesson, Traitẻ d'Ornith., P. 472.

Plilonopus purpuratus, var, Regina, id.

P.Swainsonii, G. R. Gray.

$P$. Ewingii, Gould, Zool. Soc., 1842.

Columba purpurata, Temm., Pig., pl.54?

SPEc. char. $K$. fronte et vertice roseus, hoc colore lined flava nisi ad frontem cincto; pectore virescenti-cinereo plumis bifidis, et ad apicem pallidè cinereis; fascià infrà pectorali pallidè sulphureà, abdomine medio saturatì aurantiaco, cum lunula centrali helvo-carulea, caudae rectricibus ad apicem flavis, non sine tincturd viridi prosertim in rectricibus duo. bus intermediis notandà (Gould, Proc. zool.soc., 1842, p. 18)

M. Gould a, sous le nom de Ptilonopus Evingii, donné 
la diagnose dans Proc. zool. soc. 1842, p. 18, et la figure dans Birds of Austr., d'une Kurukuru dont il dome la deseription suivante:

"Front ct liessus de la tète roses, cetle coulcur cntourée all sommet du vertex par une ligne jaune; cou et poitrine d'un cendré verdâtre à plumes échancríes, dont los pointes d'un cendré blanchâtre se dítachent et ressortent sur la fond cendré verdâtre formé par la base de chacume de ces plumes; bas de l'estomace d'un jaune soufre; rentre d'un jaune orange avec une tache médianc d'un violet bleuitre; quewe verte lermince par me bamte apicale jaune légerement teinté de verdâtre, surlout il l'extrémité des rectrices médianes ou le jaune s'apercoit à peine. $n$

En ripprochant cette deseription de celles données Har Lesson (Traté d'ornithologie, 5. 472 , l'une sous le nom dre Colombe des Nariannes, Columba rosei-capilla, lantre sous le nom de Colombe kurukuru, Columba inrmuicua, il cst diflicile de ne pas y trouver une concordance frappante.

Yoici la première de ces descriptions :

"Caiotte pourpre vif, cerdée de jaune pàle; ailes et n) dus rerts, arec des taches bleues; gorge blanchâtre; "joues grises; thorax et devant du cou gris rour; mi"licu du ventre rose vineux; ventre et couvertures in" réricures orangé ; queue blanche en dessous. ")

Nous pensons, ainsi que nous l'avons déjà dit au sujet du K. Su'tunsonii, que cette identilication est erronow wn fout proint, d'abord sous le rapport de la localité, ar andividu vient des Mariannes, tandis que le 
Swainsonii ne se trouve nulle part en dehors de la Notw velle-Hollande; ensuite sous le raprort de la coloration, qui offre de notables différences; et enfin sous le rapport de l'organisme, les ailes de cette espèce étant presque arrondies el rentrant daus la forme el la dimension de celles du Purpuratus, sans parler de la taille bien moindre.

Nous donnons, au surplus, la description détaillée faite sur le mème individu qui a servi de type: à Lesson, et qui fait partie de la riche collection du Muséum d'histoire naturelle de Paris, auquel il a été rapporté par Quoy et Gamind, chirurgyiens de l'Astrolabe, commandée par le capitaine Freycinet, en 1829:

Calotle lilas foncé, encadrée d'une bande jaune blanchâtre formant sourcil au-dessus de l'ail; quelques plumes de mème couleur que la calotte se remarquent à la base de la mandibule inférieure; nuque, joues et bas de la partic antérieure du cou, ainsi que l'estomac, d'un gris mat se détachant sur un fond rert-lirmàtre qui forme la base de chacune des plumes pectorales: une bande d'un violet noirâtre au bas de l'estomac; tout le reste du ventre et réģion anale d'un jaune orange; flanes et tarses vert olive; dessus du corps vert à reflets bronzés foncés; fines bordures jaunes aux rémiges primaires et secondaires; moyennes couvertures avec un oil ou miroir central d'un beau vert émeraude d'aitant plus remarquable, que le vert qui l'entoure est olivâtre: et à reflets bronzés très-intenses; queue à large bande apicale, de deux centimitres de hauteur, d'un blane: 
grisatre sale, heauconp plus clair sur les harles internes de chacune des rectrices.

Longueur totale. ....... 20 centimètres.

Les différences de coloration qui se remarquent entre cette espere of relle de Swainson sont faciles à saisir, les couleurs en général ("n ctant plus sombres ot beaucoup moins vives que celles le cefte derniere espece que Lesson n'avait pats voe en nafure et qui lui a fait defaut comme terme de comparaison. Le Suainsonii se rapprocherait davantacre du Clementinur mals outre que la partie anférieure du corps en est moins jaunatre, il y a entre cux une diflérence constante dans la forme ef la longhueur relative des ailes, sultisante pour les distinģuer l'une de l'autre, crelles de notre espice étant plas rourtes de deux centimètres et demie.

Voici maintenant la description donnce par Lesson sous la nom ate Colombe kuruluru, Colomba purpurata, et que nous transcrivons ici :

"Thte purpmine, bordée le jaune; ailes et dos verts, "poncturis de bleu; queue vert bleu, lordée de jaune; n worge jaune; llorax wris jaune; ceinture jaune; mi"lieu du ventre vineux; ventre orangé; couvertures " inféricures jaumes. Queue brune en dessous. De Timor, ") par Maugé. ")

Il en est de même d'un autre individu provenant de la même localité, rapporté par le même voyageur de l'expédition ans ferres anstrales du cap Baudin, qui se

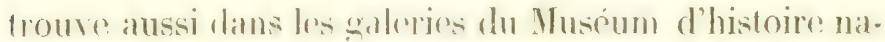
turelle de Pario. Lin wiri la deseriplion rue nous don- 
nons, parce quelle presente quelques dillórences, et indique seulement une variété :

Plaque occipitale lilas, plus foncé sur les bords, encadréc d'une fine rair à peine sensible, jannitre; tìte, cou, haut du dos, ('stomac et partie supéricure du ventre

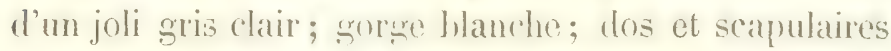
d'un rert olive it reflets bronzés; moxernes couvertures a refots blewitres ; samdes couvertures of remiges primaires, frangées larementent à l'intérien de reflets bron-

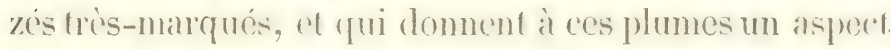
de brun rougeater ; les rectrices de mème couleur aver une bande apicale hane jaunatre; une: tache d'un noir violacé oceupe le milien de l'aludomon dont lo bats est olivatre; regron anale jaune joncuulle fournant au minium à l'extrémité des plumes.

Longueur totale. ....... 20 centimètres $1 / 2$.

Enfin, nous n'hésitons pas à rapporter à la même

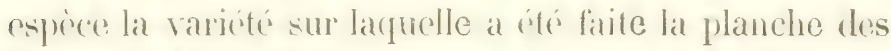
Pigeons, 3i, de .I. Temminck, sous le nom de Colombe kuruliuru, Colomba purpurata, lath. (dont nous l'avons séparée dans notre article sur cette espèce), et dont il

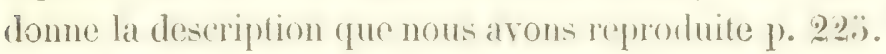

Nous croyons qu'examinant lrop) légrimement l'exrntplaire qu'il a eu entre les mains à cette ćpoque, il anra néglige de ronstater la présener de la lacho violacée qui se retrouve sur le roseicapilla ou Exvingii, comme sur le Swainsonii et le Clementina.

Resterait donc la question d'habitat, car s'il était prouve que l'Ewingii ne se touve nulle part ailleurs 
guim Australie, an l’a observi M. Fould qui lui assigne le Pont-Vssington, notre assimilation serail sans valeur; malis alors encore le roseicrpilla de Lesson n'on derait-il pas moins être conservé comme espèce distincte.

\section{KURUKURU DE CLEMENTINE.}

Kuruliuru Clementina, O. des Murs et Fl. Prévost.

Ptinolope de Clementine, Hombr. et Jacq., pl. 29, 4 g. 3, Voy. au pôle Sud.

Spec. cran. K. viridis; pileo hyanthino-purpurescenti, lined flarn stricti circumscripto; capite, collo pectoreque anterioribus cinereo-albidis, posterioribus flavidis; tegminibus alarum flaro marginatis; seapularibus speculo pulurescente-violaceo notatis, rectricibus flavo, margine virescenti, apice fasciatis; latcribus olivescentibus; pectore inferiore fascia violaced notato; abdomine medio longitudinaliter astaneo-rubido; crisso flavo.

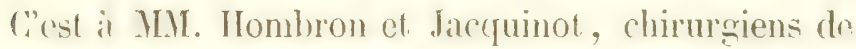
la Zibie, que l'on doil la premiire figure de celle espere dont plusieurs individus étaient depuis longtemps au Muscom d'histoire naturelle de l'aris : e'est le $n^{\circ} 3$ de la pl. 29 de l'Atlas de leur Voyage au prile Surl, sur larpuclle elle porte le nom que ces voyagreurs lui ont domnc, ot que nous lui conservons, de Clémentine, en souvenir d'illustres infortunes. Quoiqu'ils n'en aient public ancune diagnose soit latine, soit francaise, nous y suppléerons par la description suivante:

Catotle d'un pourpre violet, dípassant l'angle externe

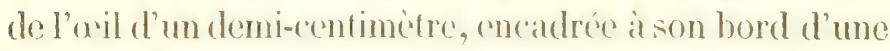
éfroite bande orangée se perdant dans la nuance vioIette; tète, coti, estomac ef poitrine d'un gris blanchàtre, plus pale el presque blane an menton et a la base de la 
mandibule inferreure, jaunatre sur la nurque et le derrière du cou; plumes de l'estomac échancrées, leur ćchancrure laissant voir pour fond la teinte jaunatre de la première moitié de la plume que chacune d'elles recouvre, en sorte que leurs pointes gris pâle se dessinent assez nettement sur ce ton jaune; dos et petites couvertures alaires d'un vert à reflet légèrement bronzé ou olivàtre, les moyennes et les grandes couvertures d'un brau vert métallique finement frangé de jaune; les trois ou quatre plus rapproches du corps portant sur le còté extrime de leur page interne, à découvert, une jolie tache ou miroir d'un violet bleuatre d'aspect pulvérulent; rectrices et rémiges d'un vert foncé métallique; les secondaires noiràtres intérieurement, les primaires extérieurement, les rectrices terminées à leur pointe par une large bande jaune légèrement frangée de verdàtre; lancs rert olive; le gris de l'estomac: terniné, au sommet de l'abrlomen, par une tache ou plastron d'un violet noirâtre, reproduisant parfois chez quelques individus, et d'une manière à peine sensible, des reflets qui rappellent le ton pourpré de la calotte; le milieu de l'abdomen dans toute sa longueur, à partir de cotte tache, d'un rouge marron se nuançant avec le jaune serin qui termine la dernière moitié des convertures fémorales, et qui teint la région anale, ce même jaune se terminant en orangé foncé et presque rouge de saturne, sur les couvertures inférieures de la queue: les remiges, (comme les rectrices, d'un beau gris de fer en dessous; la bande jaune appicale de ces dernieres pavaissant blanche frangée de jaunâtre ì chacune d'elles: 
hec noir à sa base, d'un blanc verdâtre à sia pointe; pattes d'un brun orangé.

Quelques individus ont l'estomac plus gris, d'autres plus jaunâtre.

La longueur totale des premiers est de. . 22 rentimetres.

Celle des seconds, de........ . 24

Les ailes, assez aizuës, arrivent à 3 centimètres de l'extrémité de la queue.

\section{KURUKURU DE MERCIER.}

Kurukuru Mercierii, O. des Murs et Fl. Prévost.

SPEC, crar. K. viridis; capite à naribus usque ad nucham inferam purpureo-violaceis, absque lined circulari; maculd commissurali latd concolore; corpore reliquo anteriore cinereovirescenti; dorso viridi-olivescente; legminibus alarum et majoribus tectricibus, illis caruleis, flavo marginatis; remigiis viridi-nigrescentibus; thorace abdomine olivescentiflaris; abdomine inferiore crissoque flavo-junquillaceis; canda basi grised, medio viridi, reliqua grisescenti, apice flavo fasciutid. Liostrum basi nigrum apice corneo; pedibus rubris.

Celte jolie espèen, qui lint aujourl lui partie de la collection du Huséum d'histoire naturelle de Paris, y a été apportie en 184.8, sous le nom de Colombe kuruliuru it calote pourpre, par Mr. Hercier, anquel nous la dédions, attaché au Jardin botanique; en voici la description:

Tout le dessus de la tête, depuis les narines jusqu'au bas de la nuque, d'un carmin vif violacé, bordant la paupiere superiemre ef oceupant la moitic de la surface du lorum, sans aucun encadrement ni ligne circulaire semblibble i celledes autres liurukurtis. Unelarge lache de meme couleur se trouve à la base de la mandibule infirieure, el yoceupe un espace d'm rentimelre ren hauteur ; 
tout le reste supérieur du corps, depuis les jones jusqu'à l'estomar, d'un gris légèrement verdàtre, plus pàle et presque blanchâtre sur les joues, plus verdàtre sur les épaules, légerement jaunâtre à la gorge; toutes les plumes pectorales échancrées et grises dans la première moitié de leur longrueur; elles se dessinent done en points ou stries d'un blanc jaunâtre sur un fond gris, au rebours du Sucainsonii, dont les stries se dessinent en gris sur un fond vert; dos et convertures alaires d'un joli vert olivitre, borte de jaunâtre sur ces dernières; les moyennes a prindes couvertures les plus rapprorhies du corps ornies d'un heau miroir bleu encadre d'une frange jauneserin; rémiges primaires noires, secondaires d'un vert foncé sur leur page extérieure et noires sur l'autre; estomac et ventre d'un jaune olivitre, lew: harbules de charune des plumes de ces parties so terminant à leur extrémité de jaune; région anale et couvertures inferieures de la queue jaune jonquille; quene légierement arrondie; les rectries grises à leur base dans le premicr tiers de leur longueur, d'un vert foncé rers le milieu, d'un gris sale dans le dernier tiers jusqu’à leur pointe qui est frangée de jaune; tarses enplumes dans presque toute leur longueur; bec noir ì sal base, couleur de corne olivitre i la pointe; pattes rouges.

Longueur totale......... 22 centimètres.

Ailes arrivant à 1 centimetre $1 / 2$ de l'extrémité de la queue et de forme aiguë.

IIabit. Iles Marquises, Noukiva, ou l'individu mique. a été tué par M. Mercier, dans la vallée de Mohana, sur un figuier dont cet oiseau mangeait la graine. 
Notre genre Kurukuru se composerait done, quant à présent, des espèces suivantes:

$1^{\circ}$ Kurukuru purpuratus, O. des Mlurs et Fl. Prévost.

Columba purpurata, Lath.

C. viridissima, Temm., Pig., pl. 35 .

Ptilonopus purpuratus, Swains.

$2^{0}$ Kurukuru superbus, O. des Murs et F1. Prév.

Columba superba, Temm., Pig., pl. 35.

Ptilonopus superbus, G.-R. Gray.

5. Kurukuru Temminckii, O. des Murs et Fl. Prévost.

Columba superba, famina, Fl. Prévost et Knipp, Pig., t. II, p. 77, pl. 42 .

Ptilonopus superbus, G.-R. Gray.

$4^{\circ}$ Kurukuru xanthogaster, O. des Murs et Fl. Prév.

Columba xanthogastra, Wagl.

C. purpurata, Temm., pl. col. 254 .

C. diademata, ibid., texte.

Ptilonopus flavigaster, Swains.

P. xanthogaster, G.-R. Gray.

$5^{\circ}$ Kurikuru Dupelit-Thouarsii, O. des Murs et Fl. Prév.

Columba _____ Néboux, Voy. au pôle Sud, pl. 29, f.. 1.

C. kurukuru purpureo-loucocephalus, Hombr. et Jacquinot.

Ptilonopus leucocephalus, G.-R. Gray.

P. Emilia, Lesson.

P. purpureo-leucocephalus, G.-R. Gray.

$6^{\circ}$ Kurukuru Samoensis, O. des Murs et Fl. Prév.

Ptilonopus - - - , J. Verreaux, mss.

Colombe de Vincendon, \}Iombr. et Jacq., Ann. sc. Columba kurukuru superba, $\}$ nat., 1841 .

Ptilonope de Marie, id., Voy. au pôle Sud, pl. 29, firg. 2.

$7^{\circ}$ Kurukuru Taütensis, O. des Murs et Fl. Prév.

Columba kurukuru, var. Taïtonsis, Less.

Ptilonopus Taïtensis, G.-R. Gray. 
So Kurukuru Nebonxii, O. des Murs et Fl. Prér., Voy de la Vénus, pl. $\%$.

$9^{\circ}$ K. Swainsonii, O. des Murs et Fl. Prév.

Ptilonopus purpuratus, var. Regina, Swains.

Columba purpurata, Temm., pl. col. 254, Jard. et Sellb., Ill. orn., vol. II, pl. 70.

Ptilonopus Stoainsonii, Gould., Pr. zool. Soc., 1842, P. 18. Nec Columba roseicapilla, Less.

$10^{\circ}$ Kurukuru roseicapillus, $\mathrm{O}$. des Murs et Fl. Préष.

Columba roscicapilla, Lesson.

C. purpurata, id., Temm., Pig., pl. 54?

Ptilonopus purpuratus, var. Regina, Lesson.

P. Ewingii, Gould.

$11^{\circ}$ Kurukuru Clementina, O. des Murs et Fl. Prév.

Ptilonope de Clémentine, Homb. et Jacq.

$12^{\circ}$ Kurukuru Mercierii, O. des Murs et Fl. Prér.

$13^{\circ}$ Kurukuru roseicollis, O. des Murs et Fl. Prév.

Columba , Wagl.

C. porphyrea, Temm., pl. col. 106.

Ptilonopus erythrocephalus, Swains.; Knipp et Prér., Pig., pl. 4.

$P$. roseicollis, G.-R. Gray.

Dont les plumes pectorales sont également échan. crées, mais dont les barbules latérales du rachis s’arrondissent à leur extrémité au lien de s'allonger en pointes. 


\section{PLANCHE VIII.}

\section{COLOMBI-GALLINE DES GALLAPAGOS.}

Columbi-Gallina Gallapagoensis (Néboux), Rev. zool., if fo, pag. 290.

Zenaïla Gallapagoensis (Gould), Zool. of the Beagle, pl. 46 , pag. 115.

Cinar. spec. Zen. corpore toto suprà rufo-cinerascente ef parumper vinaceo; collo imo lateralique viridi-aurato purpureoque metallicè splendente; gutture et pectore vinaceis; remigibus primariis nigris albo anguste marginatis; secundariis externe cinereis; tectricibus minoribus in margine externo speculo nigro notatis; illo intus albo cincto; mediis in prima parte externè nigris, secundâ albis, ultima concoloribus; majoribus omnini nigris, in medio albo striatis, apice rufis; alula nigra; regione periophthalmica nigrd; auribus albis, strid nigrả infrà cinctis; gulà nigro tenuissimè squamatâ; 4 rectricibus lateralibus paginà externâ cinereis, albo marginatis, nigro prope apicem lati fasciatis; mediis apice cinereis : rostro nigro-cinerascente, pedibus carneis.

Cette espèce de Colombidé a déjà été figurée par M. Could, dans la zoologie du voyage du keagle, j). 16.

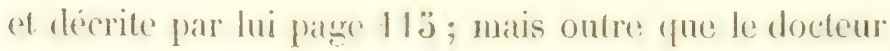
Nehoux l'avait déja nommée décrite en 18 1.0, dans la Revue zonlogigue, page ?90, celui des individus rap)portes par celui-ci que nous figruroms est d'un dẹce plus adulte et l'un plumagre plus parfail que celui du natu-

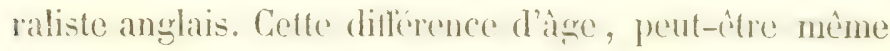
de sexe, en amenant une dans la livrée, il ne sera pas sans intérêl de comparer l'un et l'autre exemplaires. 
Description. Yous reproduisons les caractires princ1paux de cette espèce d'aprés la note insérée par le docteur Néboux dans la Revue zoologique:

"Bec noir, elfile, assez long. Au-dersous de l'orbite, " on remarque deux lignes noires qui partent de chà") cune des mandibules et vont se terminer à l'oreille " qu'elles embrassent dans l'intervalle qui les sépare: "li les plumes sont blinchitres. Toute la partie supé" rieure du corps est d'un gris roux. Au niveau des "ailes et a la base du cou seulement cette couleur prend ") un aspect métallique à reflet pourpre ou vert doré. "La gorge, hanchàtre sous la mantibule, passe au vi") neux. Poitrine vineuse. Ventre, anus et dessous des "ailes gris cendré. Rémignes primaires noires, lisirrées "de blanc. Couvertures moyemnes blanche's sur le hord ») externe. Rectrices de la même couleur que le corps ; " elles présentent une zone d'un beau noir à 2 cenn timctres de leur terminaison. Tarses rouges, ongles ") noirs. "

Nous ajouterons, pour compléter cette description succincte, que les petitescouvertures alaires, de la même couleur que le dessus du rorps, portent sur chacune de leurs plumes, au bord at lout he long de leur page externe, une srande tache noire formant la moitió d'une écaille, encadrée à sa hase el le long de son bord intérieur d'un liseré hlanc; que les couvertures moyennes ont la mème page noire à sa naissance juscuáan tiers de sa longueur, blanche dans le deuxieme tiers el roussitre dans sa dernière partie; que les couvertures dorsales sont noires dans la plus grande partie de leur sur- 
face; que ce noir est encadré de roussâtre et divisé dans foute la longrueur de la partie machidienne qui les fraverse par une ligne blanche s'blargissint i la lase; enfin que les rémigres secondaires sont grises extérieurement; que les quatre rectrices latérales, de la mème couleur, sont finement lisérées de blanc, et que les couvertures supérieures de la queue présentent un faux reflet métallique verdâtre.

Longueur totale. ........2 21 centimètres.

- de la queue. $\ldots . . .6 .63$

Voici maintenant la diaggnose domnée de l'individu du Beagle par MI. Gould:

" $Z$. vertice, cervice, dorso caudceque tegminibus obscure ) fuscis vinaceo tinctis; dorso nigro-guttato; alarum ") tegminibus fuscis, plumi singula pallidi vinaceo-fusco " terminati, pogonii utrinsque margine; macula oblonga " magna nigra, lineâ alba separata; remigibus primariis. " et secundariis nigrescenti-fuscis, cinerascenti-albo an"gusti marginatis; caudâ fuscescenti cinereo ad apicem ") fascia latâ irregulari nigrri; loris linerique angusta suprà " ct infrà oculari nigris pallidè fusco mixtis; gulii pecto"reque vinaceis, colli lateribus arato tinctis; crisso, " cauderque tegminibus inferioribus cincrascentilus; rostro "nigro, pedibus rufescenti-aurantiacis. "

Il suflit, on le voit, de rapprocher les deux descriptions comme les deux figures pour juger des diflérences qu'elles présentent.

Ainsi, la plus notable de ces différences réside dans l'absence presque compliete, chez l'indivilu du Bengle, 
de la large tache noire du lorum dont on entrevoit a peine la trace dans un trait noir finement tiré de la base de la mandibule inférieure à l'angle externe de l'oeil, dans l'absence de l'oreillon blanc, et de la ligne noire qui l'encadre a sa partie inférieure, remplacée ici par une lache brune; ensuite les rémiges secontaires, d'un beau gris cendré chez notre individu, sont d'un brun noirâtre chez celui du Beagle: enfin les rémiges et les rectrices qui, chez celui-ci, sont à peine borlées de brunâtre ou de gris obsrur, sont, chez l'autre, borlées dans toute leur longueur d'un blanc bien prononcé.

Toutes ces différences, sans parler dı ton général beaucoup plus sombre et plus foncé chez l'individu du Beagle que chez celui de la Vénus, prouvent surabondamment que ce dernier représente bien un mâle adulte tel que se sont trourés tous ceux rapportés par l'expédition que nous avons eu occasion d'observer, et que le premier représente tout au plus une femelle adulte de la mème espèce.

M. de La Fresnaye, dans la Rer. zool. 1840, pag. 322, dii, au sujet d'un individu de sa collection qu'il a comparé avee trois individus de la Vímus : "J'ai remarqué " entre eux une différence de taille tout à fait notable. ") ce qui semble indiquer deux races de la mème espece "plutôt qu'une différence de sexe, le plumage étant ab)"solument coloré de mème chez les quatre individus. " el paraissant un plumage adulte, quoique deux d'entre "n eux soient presque d'un liers plus forts que les " deux autres. " Celle remarque, exacte en elle-même, n'avait pas échappé au docteur Néboux, et nous ne OISEA UIX. 
pouvons que la contirmer. Au reste, celte diflérence de laille, de mène que les differences de coloration que nous venons de signater entre l'individu du Beagle el felui de la Vémus, n'ont rien de plus extraordinaires et sont relativenent les mèmes que celles qui s'observent fréfuemment dans la Columba Fitzroy, dont on a longtemps forme deux espieces sous le nom de C. Denisca (Temm.), pl. col. 502, et C. Arancana (Less.), Voy. de la Coquille : car nous croyons que c'est à tort que M. G.-R. Gray persiste encore, dans son Genera of birds, à maintenir la preniere de ces dénominations isolée spéciliefurment des deux dernirres, toutes les trois, selon nous, se rapportant à une seule et même espèce.

(quant a la comparaison que fait M. de La Fresnaye de notre esprece arec C. Montana (Limn.) qui est la mème. que C. Martinica (Temm.), efle repose sur une donné plus spécieuse: que réselle; car si, par le fond principal de sal roloration, dhe rappelle eflectivement un peu re lype americain, elle s’en éloigne considérablement par la forme de son ber effile, qui l'a fait placer par M. Could dans le genre Zenüde de M. Ch. Bonaparte, adoptédepuis par M. Gray.

C'est dans l'ile Saint-Charles de l'archipel deGallapagos que le docteur Néhoux a découvent les individus par lui rapportes. Ses observalions sur ces oiseaux étant les mènes que redles rommmuniqués à II. de La Fresnaye

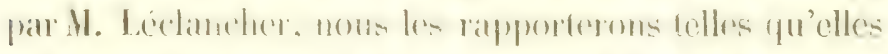

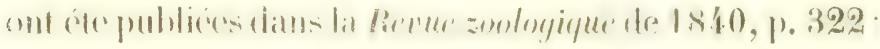

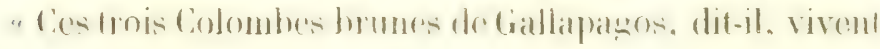


\#) it terre, et sont si peu sauvages que les déportés de "Guayaquil qui habitent les Gallapagos les tuent an n moyen d'une perche, et en assez grand nombre pour "les vendre par paquets comme des Alouettes, et à ") assez bas prix. ") Elles se rencontrent dans tontes les parties rocheuses de l'Archipel.

\section{PIUVIER DE: WILSON.}

Charadrius IVilsonii, Ch. Bonap., Suppl. it IV ils, 1. IX, pl. 75, fig. 5 .

Trouvé à Monterey (Hanto-Californic).

\section{HERON DE LA TOUUSIANE.}

Ardea Ludoviciana, Gm., pl. enl. 908, 909, 915.

Descend au midi jusqu'a Cayenne, St.-Thomas, PortoRico, et dans presque toutes les Antilles. Trouvé par le docteur Néboux aux Gallapagos, d'où on ne l'avait pas encore rapporté.

\section{CANARD DE LA CAROTINE}

Aix Sponsa, Boié.

Anas - - , Gm., pl. enl. $9^{60}, 9^{81}$.

Dendronessa - Rich. et Swains.

Indiyué par Richard et Swainson, comme émigran! en hiver aux États-Unis et peut-être au Mexique: trouvé à Monterey par le docteur Néboux qui l'en a rapporté. 


\section{PLANCHE IX.}

\section{NODDI CENDRE.}

Stolida cinerea (Néboux), hev. zool., oct. 1840, p. 291. Anous cinereus (F1. Prévost et $\mathrm{O}$. des Murs).

CHAR, SPEc. An. corpore cincreo; suprà fusciùs infrà dilutiù, capite et fronte sericeis albescentibus; macula oculari alba; remigibus nigrescentibus; tegminibus brunneis; cauda furcata: rostro ac pedibus nigris; palmis flavescentibus.

Voici la description que le docteur Néboux a donnée de cet Oiseau dans la Rev. zool. d'oct. 1840, p. 291. » Sterne cendré. (Sous-genre Noddi) : Bec noir, grêle. »Iris noir; paupières noires. Tète et front gris blan") châtre, ayant l'aspect satiné. Col et dos cendré foncé. ") Gorge, ventre et dessous des ailes bruns. Queue " passablement fourchue, de la même couleur que le " manteau; la seconde penne est la plus longue. Tarses ") très-longs et noirs : membranes interdigitales jau) nâtres.

»Longueur totale, 24. centimètres.

) Habit. Pris à la mer dans l'océan Pacifique (hémi) sphère boréal). ")

Ce Noddi a les plus grands rapports de coloration dans l'ensemhle avec le Noddi (Anous) Melanogemys, diguré par G.-R. Gray dans son Gen. of $B$. en juin 184.6, pl. 18\%. Comme celui-ci, il a une teinte générale cendrée plus ou moins claire à la tête ct au-dessous, plus ou moins foncée en dessus; comme chez lui aussi, les plumes de la tive del front prement am aspeet soyeux. 
Mais il en differe d'abord, organiquement, par unc taille moindre, le Melamogenys ayant pris de 4 centimètres de plus; par un bec beancoup plus en rapport avec celui des vrais Sternes, et n'ayant pas trace de l'inflexion si prononcée chez ce dernier ; par l'échancrure, très-prononcée de sa queue, et sous le rapport de la ptilose par un gris plus franc et par conséquent moins noir ; par l'absence de cette dernière couleur aux joues ; par une tache d'un bleu pur qu'il porte à l'angle interne de l'œil, à la hauteur du sourcil, et par la coloration des petites plumes garnissant le bord des paupières. qui en font un cercle, encadrant l'œil, noir dans la partie interne et blanche dans l'autre; en outre par la coloration des palmures, qui sont noires che\% le Melanogenys.

Notre espèce fait la onzième du genre.

\section{PLANCHE X.}

\section{MOUETTE A QUEUE FOURCHUE.}

Larus furcatus (Néboux), Rev.zool. 1840, p. 290.

Char. spec. L. supra cineraceus, capite colloque fuliginosè no. grescentilus; subtùs albus; striga postoculari et basi frontali albis; palpebris aurantiis; tarsis palmisque rubris.

Le docteur Néboux a donné dans la Rev. zool., octobre 1840, p. 290 , la description suivante de cette belle espèce de Monette :

"Tète el la presque totalité du cou gris brun. Deux ") petites taches blanches arrondies embrassęnt symé") triquement l'extrémité postérieure de la mandibule 
") supérieure. Manteau blanc gris. Poitrine, ventre, ") région anale, dessous des ailes blancs. Niles plus "longues que la queue de f centimetres. Rémiges pri" maires noires sur le bord externe et le bord interne.

"Couvertures moyennes blanches; les supérieures ar" doisces et bordées de blanc. Queue tris-fourchue et " hlanche. Les deux rectrices plus longues qu'elles ne ") le sont d'habitude dans ce genre d'Oiseau. Bec très") arqué, noir à sa base et blanc à l'extrémité. Iris " rougres. Paupières orangies. Tarses et palmes rouges. ") Ongles noirs. "

Longueur totale........ Go centimètres.

Mabit. Prise dans la rade de Monterey (Hante-Calli. fornie).

Malgré la grande difliculté que l'on éprouve généralement à distinguer spécitiquement les nombreux individus qui composent le grenre Larus, i cause des grandes analogies et aussi des frúruentes variations qu'ils présentent dans leur coloration, notre espèce se sépare à premiere vue de ses congeneres par la couleur orangre de ses paupières et la couleur rouge de ses tarses et de ses palmures, surtout par le miroir blane mat qui orne la partic supérieure du bee on hasale du fromt, quoique le rouge des pattes soit commun à plus d'une espèce, entre autres aux Lar-Hamatorluynchus de King et Bonapurtei de Richard ot de Swamson. Nous ne parlons pats de la tache blanche formant demi-cercle à la paupière inférieure, parce que cette tache se retrouve tantòt en bas, comme ici dans le harus fromblinii (Rich. el sw.), 


\section{$-279-$}

lantòt en haut, comme dans le $L$. Bomapurtei. Les deus autres caracteres les plus saillants ef les plus remarquables chez notre espece sont la forme arquee du bee qui rappelle un peu cependant celui du $L$. modestus de T'schudi, et la forme profondément fourchue de la quueur. qui ne se retrouve que chez les vraies Sternes.

\section{PÉLICAN BRUN.}

Pelecanus fuscus, Gm., pl. enl. $9^{5} \%$.

P. Carolinensis, Gm.

Commun anx Antilles; tronvé sur la còte occidentale: de. I'Amérique néridionale, au P'érou; ohserve sur la mème côte de l'Amérique septentrionale par le docteur. Néboux, qui l'a rapporté de Monterey. 



\section{INDEX}

DES ZSP'ECS DÉGRTES OU INDIQUÉES ET DES TOMS GÉNGRTQQES et SPÉchfipues cités daAs Ge trayall.

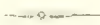

Pages.

Aix sponsa ........... 275

Anas _........... 275

Anous cinereus. .......276

A. melanogenys........ 276

Ardea Ludoviciana. ...... 275

Arremon biarcuatus. . . . 216

Bec-croisé leucoptère. ..... 204

Bruant à double eroissant. .. 216

Cactornis grimpeur. ......204

C. scandens. ........ 204

Camarhynchus cineraceus. . 209

Calliste rufiveriex........212

C. labrarborides.......2 213

C. Teclancherii. .......214

Canard de la Caroline. .... 27j

Caracara vulgaire........ 181

Casse-noix.......... 20:3

Certhia coccinca....... 157

C. obscurt....... 180,191

Charadrius Wilsonii....... 275

Cinnyris unea........ 18.5

Cioccolorus. ........ 210

Coccolliraustes ....... 200

Colombe kurukuru. ..... 221

Columba kirukuru. ......221

C. jurpurata. - 218, 219, 222, 238 , $256,259,268$

Colombs viridissime...... 229

Columba viridissima. 222, 227, 268

() ISLIII:
Pages.

Columba supcrba...... 231, 268

Colombe à diadème. . . . . 239

Columba diudemata. . . 238, 268

C. superba, fominr. . . 234, 268

C. xanthogustra..... 238, 268

C. Dupetit-1houarsii. . 241, 268 Colombe kurukuru leucocéphale. 243 Columba kurukuru purpureo-

leucocephalus.. . . . 241, 268

Colomise de Vincendon. . 247, 268

Columba kurukuru superba. . 247.

268

Colombe de Taïti...... 252

Columba kurukuru, var. Tahi-

tensis........ 251, 208

C. rosicapilla. . . 250, 259, 260

C. roseicollis......... 269

C. porphyra, ....... 269

Colombj-galline des Gallapagos. 270

Columbi-gallina Gallapagoensis. . 270

Corvus caryocatuctes .... 203

(. Bierlarii........ 203

Curvirostra leucoptera. . . . 20!

Crucirostra leucuptera. . . . 204

Cyanocorax de San-Blas. .. . 200

C. San-Blasianus. ...... 200

C. de Beechey............ 20?

Cypsnagra...........211

Dendronessa sponsa..... . 275

Drepanis coccinea. . . 185,187

19 
Drepanis lucida....... $\begin{array}{r}\text { Pages. } \\ 192\end{array}$

D. obscura. ........ 191

I), olivacea......... 192

Emberiza biarcuata. ..... 216

Erythaca Wilsonii...... 198

Esclave des palmiers. . . . . 211

Étourneau militaire. . . . . 203

Euplectes. .........200

Falco cheriway. . . . . 181

F. Brasiliensis....... 181

F. Tharus.......... 181

Fauvette de Wilson ..... 198

Fringilla biarcuata......210

f. noctis..........2 210

Cieai de San-Blas. ....... 201

Géospiza........206, 207

Géospiza fuligineux...... 208

Geospiza fuliginosa......208

Grallarie squammigère.....198

Grallaria squammigera. .... 198

lir. rex........... 198

Gr. imperator........ 198

Grallarie de Guatémala. . . . 199

irallaria Guatemalensis. . . . 199

(iros-bec azulan du Brésil. . . 210

Gros-bec bleu des Etats-Unis. . 210

Guiraca cendré. ...... 209

G. cinerea.......209, 211

Hémignathe. . . . . . . 183

Hemignathus. . . . . 183, 185, 189

Hémignathe brillant. . . . . 192

Hemignathus lucidus. 187, 190, 191,

192

H. obscurus. ..... 189, 191

H, olivaceus......... 192

Héorotaire vestiaire..... 186

H. écarlate. ........ 187

Héron de la Louisiane. . . . 275

Heterorhynchus. ....... 183

Heterorhynchus olivaceus. 189, 191,

192

Hirundo concolor. ..... 182

H. modesta......... 182 pages.

Kurukuru. .......219, 220

K. à couronne pourprée. . . . 222

K. purpuratus ..... 222, 268

K. Poukiobou ........ 231

K. superbus . . . 231, 268

K. à diadème. ........ 238

K. xanthogaster .... 238, 268

K. Dupetit-Thovars ..... 241

K. Dupetit-Thouarsii . . 241, 268

K. de Samoa........ 247

K. Samoensis. . . . 247, 268

K. de Taîti. ........ 251

K. Tartensis ...... 251, 268

K. de Néboux........ 253

K. Nebouxii ...... 253, 269

K. de Swainson ...... 256

K. Swainsonii ..... 256, 269

K. des Mariannes ...... 259

K. roseicapilla..... 259, 269

K. de Clémentine .....264

K. Clementinz. .... 264, 269

K. de Mercier........266

K. Mercierii ...... 266, 269

K. de Temminck. ..... 234

K. Temminckii...... 234, 268

Lamprotes ........ 211

Larus Bonapartei. . . . 278, 279

L. Francklini....... 278

L. furcatus ........ 277

L. homatorhynchus..... 278

L. modestus. . . . . . . 279

Leucopygia . . . . . . . 211

Loxia leucoptera. ...... 204

L. falcirostra. ...... 204

Lucifers. .........196

Mellithreptus vestiarius. . . 187

Mellisuga Costo......194

Ministre .........246

Mniotilte à sourcils. ..... 197

Mniotilta superciliosa. .....197

Mniotilte d'été ........ 197

Mniotilta restiva........ 197

Motacilla pensilis ...... 197

M. flavicollis. ....... 197 
Iotacilla sialis....... 198

M. canadensis ...... 197

M. albicollis ........ 197

Mouette à queue fourchue. . . 277

Nectarinia anea ...... 185

Nectarinia........... 185

Noddi cendré. ........ 276

Noddi melanogenys. . . . . 276

Nucifraga caryocatactes ....203

N. guttata .........203

OEnanthe sialis....... 198

Oiseau-mouche de Costa. . . . 194

Ornysmiz Costæ....... 194

O. ornata......... 196

O. strumaria........ 196

O. petasophora....... 196

o. Vieillotii. ........ 196

passerines ..... 214, 216

Passerine de Léclancber. . . . 214

Passerina Leclancherii. . . . 214

Passerine pape.......2 216

Pélican brun........279

Pelecanus fuscus. ...... 279

Pelecanus Carolinensis.. . . 279

Père-noir. . . . . . . . 208

Pica san Blasiana. .... 200

P. Beecheii. ....... 203

Pie Geng. ......... 202

Piezorhina ........ 211

Pitiosittacus Americarus. . 204

Plectrophanes........ 217

Pluvier de Wilson...... 275

Polyborus Brasiliensis...... 181

P. vulgaris.......... 181

Procnopis atro-cerulea. . . . 212

Procné modeste. ...... 182

Procne modesta. ....... 182

Psittacin.......... 210

Ptilonope........ 218

Ptilonopus......... 218

Ptilonopus Emilia.... 241, 268

P. xanthogaster..... 238, 268

P. purpuratus . . 218, 222, 268
Pages

Ptilonopus Taïtensis. . 251, 268 P. leucocephalus ... 241, 268 P. superbus. . . 231, 234, 268 P. flavigaster. .... 238, 268 $P$. purpureo-leucocephalus. 241, 26 s $P$. samoensis..... 247, 268 Ptilonope de Marie. . . 247, 26 Plilonopus Swainsonii.. 256, 259,

269

P. Ewingii...... 259, 264 P. purpuratus, var, Regina. 259.

P. superbus, var. Regina. 218, 256 Ptilonope de Clémentine. . 264, 269 Ptilonopus erythrocephalus. . 269 $P$. roseicollis . . . . . . 26 ?

Rhimanphus citrinus. .... 197

Saxicola sialis. ...... 198

Sialia Wilsonii....... 198

Soui-mangas. . . . . . 18 .

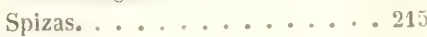

Spiza amana..........215

Spizas Tanagras ....... 215

Spiza versicolor...... 215

S. Leclancherii.......214

Sterne cendrée. ...... 27 ;

Stolida cinerea......2 276

Sturnella militaris...... 203

Sturnus _-_ ..... 203

Sylvia astiva. ....... 197

S. canicapilla ....... 197

S. childreni......... 197

S. citrinella. ....... 197

S. pensilis. ........ 197

S. superciliosa ...... 197

S. velata.......... 197

Sylvicoia ......... 197

Tachyphone .........211

Tanagra canicapilla. ... 197

T. Labradorides ........213

T. rufivertex....... 212

T. rubrigularis....... 211

T. rubricollis....... 211

T. ruficollis. .....211, 217 


\section{$-284-$}

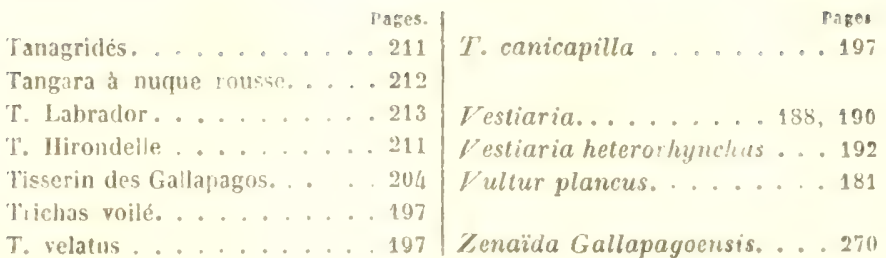




\section{REPTILES}

PA R

M. DUMERIL。

Les Reptiles que nous allons faire connaitre sont en petit nombre : ils appartiennent à un genre de Sauriens dont la plupart des espèces n'ont étć observées qu’au Chili; ce sont celles que les auteurs de l'Errpétologie générale ont désignées sous le nom de Proctotrètes ${ }^{4}$.

Ces petits Lézards appartiennent à la grande famille des Iguanes dont le corps est revêtu de lames cornées, placées en recouvrement les unes sur les autres, à la manière des tuiles. Ces écailles sont distribuées en quinconce et non par bandes ou en verticilles circulaires, et celles qui recouvrent le ventre sont arrondies ét non en plaques carrées.

Le dessus de leur tète est protégé par un grand nombre de placues symcitriques, mais disposées de di-

1 Duméril et Bibron, t. IY, p. 266, pour indiquer les pores ou les trous qui se trouvent au-devant du cloaque. De zpwxros. podex, et de тpriòs, perforatus.

Reptiles. 
verses manives dans leur arrangement. Les yeux sont constamment garnis de paupieres mobiles revotues do fines granulations; lours dents petites, legirement comprimées, sont implantées dans une rainure pratiquée sur les bords de l'une et de l'autre mâchoire; leurs racines cachées sont adhérentes aux os et par leur côté externe. Elles ne sont pas creuses et se trouvent rangcés dans un sillon qui leur forme un alvéole commun. Ontre ces dents latérales, il y en a d'autres plus petites qui garnissent la région moyenne du palais.

Leur langue est molle, charnue, couverte de papilles veloutées; elles ne peut pas rentrer dans une sorte do gaine ou de fourreau comme celle des Varans avec lesquels on pourrait les confondre, parce qu'ils n'ont pas, non plus, de crête dorsale; cependant ils n'acquièrent jamais d'aussi grandes dimensions.

Ces espèces, comme toutes celles de la Famille des Eunotes dans laquelle on les a placers, liflerent surtout de nos Lézards, parce qu'elles ont des écailles qui recouvrent la partie inférieure de leur ventre et non des plaçues carrées, disposées par bandes transversales ef régulières, mais placées en recouvrement les unes sur les autres en quinconce comme les tuiles de nos toits. T'ailleurs le phus souvent les cicailles du dos at des flance sont allongées, pointues à leur extrémité libre; elles sont surmontées d'une arête saillante qui règne sur touto lenr longueur; celles qui recouvrent les régions inférieures du cou, du ventre et même souvent du dessous de la queue, sont lisses at arrondies. Aucune des especes do ce genre ne porte, le long ru bord interne des 
cuisses, la série de pores ou de petits orifices glanduleux qui se voient dans les vrais Lézards.

A ces caractères généraux on pourrait en joindre plusients autres pour les faire distinguer les espreces qui

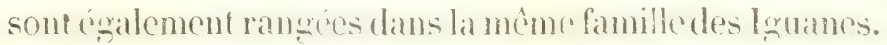
Ainsi ils different d'un grand nombre par la conformation, déjà indiquée, des écailles carénées du dessus de leur corps; les bords de leurs doigts et de leurs orteils ne sont pas élargis ou dilatés comme ceux des Anolis. Même, par une sorte d'anomalie ou d'exception dans cette famille, la partie moyenne de leur dos ne porte pas de crête ou de ligne saillante, produite par une séric de très-grandes écailles cornées et flexibles.

Leur queue, arrondic et conique, n'est pas garnie d'épines à la base; elle n'est pas destinée à s'enrouler ou à se courber en dessous, comme celle des Urostrophes; leur gorge ne forme pas un repli de la peau simulant une sorte de fanon ou de goître, et leur cou, quoique présentant le plus soment des lignes saillantrs longitulinales au dela du conduit auditif, n'oltre pas ce pli transversal qui s'observe au devant des épaules et au fond duquel on distingue de petiles granulations dans quelques genres voisins.

Toutes ces particularités, que nous avons cru nécessaire de rappeler, ont servi à distinguer ce genre des Proctotrètes de la race très-nombreuse des Iguaniens, famille dont tontes les espèces paraissent habiter les contrées les plus chaudes des deux Amériques.

Ainsi, en résumé, les espèces que nous devons faire 
conmaitre apparfiement an genre Proctotrite "l peurent être ainsi caractériscées:

Leur corps est allongé, cylindrique, légèrement déprimé, couvert d'écailles entuilées; à doights distincts, onguiculés, coniques; sans pores aux cuisses; sans crète ou ligne saillante au milıeu du dos; à queue sans épines à la base, dont lo dos est garni d'écailles carénées; sans fanon ou goître, ni pli transversal an cou an devant des épaules.

C'est un genre peu nombreux dans lequel on n'a encore inscrit que dix espèces, la plupart originaires du Chili, parmi lesquelles sont rangées celles dont la description va suivre et qui sont figurées dans cet ouvrage.

Comme ces espèces sont petites, qu'elles sont organisćes de manière à exercer des mouvements prompts et faciles; que leur bouche est étroile, sarnie de dents tranchantes of que leur palais est muni de petils crochets, tout porte à croire que la principale nourriture do ces Sauriens consiste en insectes yu'il doivent saisir et poursuivre avec une grande agilité.

P.ANCHE 1.- FIG. 1 et 2.

PROCTOTRETE DU CHIL.

proctotretus Chilensis.

Cette espèce diflère de coutes colles qui ont été jusqu'ici rapportées au mème genre parce qu'elle n'offre pas de différence dans les écailles qui recourrent les 
parties latérales du cou ou dans l'espace compris entre la tête et les épaules. On ne voit d'autre enfoncement que celui qui correspond au trou auditif, au fond duquel on distingue la membrane du tympan et cet orifice est bordé à son pourtour de petites écailles plates, comme festonnées.

Les lames cornées minces, à reflet brillant, qui recouvrent le dos et les flancs et mème tout le pourtour de la queue, surtout à la base, sont allongées, pointues et surmontées d'une forte carène saillante sur toute leur longueur, de sorte que leurs séries forment des liggnes saillantes qui paraissent se continuer et produire ainsi entre les écailles de véritables sillons longitudinaux.

Les figures qui représentent deux des principales variétés de ce Saurien sont trés-exactes, et quoique ce Reptile ait été le sujet des descriptions de plusieurs auteurs, nous croyons que les dessins soignés qui ont été exécutés sur les objets mêmes, en parfait itat de conservation, seront fort uliles pour la science.

C'est d'après plusieurs individus semblables que les auteurs de l'Erpétologie générale avaient fait connaître l'histoire de cette espèce ". On trouve là les citations qu'ils ont faites de l'ouvrage de MHI. Lesson ct Garnot dans la partie zoologique du voyage de la coquille publié en 1819, où ce Saurien se trouve décrit ou figruré sous le nom de Galéote, genre dont il diffère essenticllement, parce qu'il n'a pas de zrande crête dorsale, étendue depuis l'anus jusqu'ì la queue. On voit aussi que co Reptile a été observé et décrit par M. Wiegmann sons

'Trome IV, page 26 . 
le nom de Tropidurus dans le tome X VII des Curieux de la nature, publié en $183 \%$. Ce nom de genre ne pouvait en eliet convenir qu'a celte scule espèce à cause des carines on des lignes saillantes qui se prolongent sur la plus grande partie de la queue.

Les Planches parfaitement colorices nous dispensent de les décrire. On vera que c'est principalement par la distribution des taches que ces deux individus different entre eux, car la forme et la disposition de leurs écailles sont absolument sembables. L'arrangement des plaques qui recouvrent le dessus de la tête se trouve indiqué par la figure (1, 2.6), Les grandes écailles de la base de la queue vue en dessous avec la plaque qui recouvre le cloaque, font voir les deux pores qui ne s'observent, à ce qui paraît, que dans les mâles. Ce sont ces pores qui ont fait donner aux especes de ce genre le nom de Proctotrète.

Beaucoup d'individus recueillis au Chili par MII. Gay ef d'Orbigny sont déposés dans les collections du muséum d'histoire naturelle de Paris.

PLANCHE II. - Fig. 1.

PROCTOTRETE INTERMEDIAIRE.

Proctotretus intermedius.

Ce petit Saurien offre en effet beaucoup de rapporis, par sa configuration génírale, arec celui qui se trouve représenté sur la mème planche oi il est désigné sous 
le nom de Ventre bleu; mais il offre deux raies longitudinales jaunes ou blanches bien distinctes ot parallèles de chaque cóté du tronc. La bande inferieure plus citroite se termine à l'origine de la cuisse; la supérieure, un peu plus large, surtout au milieu, naissant à peu près à la même distance près de l'œil, se prolonge sur la queue au delà du quart de sa longueur vers la base.

Comme on a eu soin de bien représenter la distribution des placques qui recouvrent le sommet de la tète $\left(\begin{array}{ll}1 & b\end{array}\right)$, on peut remarquer que leur arrangement est tout à fait inverse de celui (qui est reproduit pour l'espece à Ventre bleu (PI. II, fig. 2.b). Ici le disque antérieur est produit par cinq flarques qui touehent une grande écaille centrale à cinq pans dont l'intérieur se trouve excessivement petit, relativement aux quatre autres; puis vient un écusson impair, allongé, qui rejoint en arrière un second disque ou cercele formé de six grandes plaques rangres ésalement autour d'unr autre plus grande qui se trouve ainsi impaire et centrale.

La figure (1.d) fait connaitre la disposition remarquable du prolongement de la peau du ventre au-dessus du cloaque dont il forme la lève antérieure. C'était probablement chez un individu màle, puisqu'on y roit indiqués les pores qui sont ici au nombre de cinq, ce qui semble caractériser le sexe de l'individu, dont la collection du Museum posside en offet un exemplaire qui est sans doute celui qui a servi de modèle au peintre. Car il n'est pas décrit dans le volume quatrieme de l'Erpatologie générale publié en 1837. 


\section{PLANCHE II. - FIG. 2.}

\section{PROCTOTRETE VENTREBLEU.}

Proctotretus cyanogaster.

Ce petit Lézard, quoique n'ayant pas de pli transversal au cou en avant des épaules, offre cependant latéralement des espaces longitudinaux couverts de petits tubercules granulés qui commencent immédiatement en arrière des trous auditifs. Cette disposition se remarque dans la plupart des espèces différentes de celle du Chili, dont la peau du cou est recouverte de grandes écailles semblables entre elles.

Celui-ci est facile à distinguer, d'abord par la couleur bleue qui tient tonte la région inférieure de la gorge, du ventre et de la queve. Le dessus du tronc est d'un brun verdatre à reflets brillants et un peu métalliques ou nacrés. Les flancs portent deux bandes longitudinales jaunes qui, naissant derrière l'orbite, se prolongent jusque sur le premier quart de la queue. Cette partie du corps est deux fois plus longue que le reste du trone; elle se termine insensiblement en pointe, et, dans les trois quarts de son étendue, olle est fortement colorée d'une teinte rouge briquetce, et comme cuivreuse ou métallique.

La figure (2.a) indique la disposition des plaques labiales, les granulations fines qui recouvrent l'une et l'antre paupières, ainsi que les plis collaires longitudinaux. 
Le dessin inserit sous le $n^{\circ}$ e). 6 montre les plaques quu revêtent le dessus de la tète. On voit qu'elles sont autroment distribuées et configurées que celles dont nous arous indirguc l'arrangement dans l'esprece qui jonte le nom d'intermédiaire. Il y a ici six plaques formant une sorte de cercle; toutes sont rangées autour d'un axe commun, mais sans écaille centrale. A la suite, on trouve un écusson impair, allongé, rétréci en arrière et beaucoup plus large en avant.

La figure (2.d) est destinée principalement à faire voir comment la partie interne des cuisses est finement chagrinée ou recouverte de petits tubercules tout à fait differents des reailles entuilese of des ranulations qui se retrouvent en outre sur les bord du cloaque.

Il paraît que les individus qui appartiennent à cette espèce ne prennent jamais une grande taille. Nous avons vu plusieus individus rapportes du Chili patr IJ. Cay ; ils étaient à peu près de la taille de nos Lézards des Sourhes. La conleur blenes'est parfaitement eonserve dase l'alcool.

PLANCHE III. - FIg. 1.

\section{PROCTOTRETE $\triangle$ TACHES NOIRES.}

Proctotretus nigro maculatus.

Le nom spécifique donné à ce Saurien par M. Wiegmann s'appliquait au mcime animal; mais il etait pour lui un Tropidure à gorge lisse (Leiolarmus); car sa description, insérée dans le travail que nous avons cité 
précédemment, ce nom parait devoir convenir à l'individu qui se trouve ici représenté.

Sa couleur est d'un brun roussâtre en dessus, avec deux bandes longitudinales plus pâles, parsemé de taches noires irrowulieres qui sont surtout remarquables sur l'occiput. Une raie noire borde le con au-dessus de l'epaule, et śrend, arec de légreres interruptions, jusqu'a l'origine des cuisses. Le dessous du corps est d'un gris pâle et sans taches.

Ce qui distingue principalement cette espèce, ce sont des cranulations qui ocrupent tout le lessous ef lebord interne des cuisses, ainsi que la lèvre postérieure du bord du cloaque, comme l'indiquent les figures ( 1 .d of 1.0$)$.

On voit les plaques syncipitales figurées ( 1 b); elles sont tout autrement disposées que dans les autres espèces. Il y a quatre écussons impairs. Deux petits qui se touchent en avant et sont encadrés par huit autres plaques disposées par paires, dont les posterieures sont les plus atendues. Vienl chsuite un grand écusson régulier et plus large en haut. Le quatrième impaire a huit pans, dont les deux intérieures sont les plus petits.

Les trous auditifs $(1 . a)$ sont bordés et précédés de quatre grandes écailles dentelées et saivies de granulations limites entre deux replis saillants et ceailleux de la peau du cou.

La queue est longue : elle dépasse de moitié l'étendue du tronc. Très-grosse à la base, elle diminue insensiblement pour se terminer en pointe aigue. 
Les pattes postérieures sont du double plus longues que les antérieures; car, dirigées en avant du côté de l'épaule, les doightópasseraiont de beaucoup cette région; tandis que les membres de devant, étendus le long du ventre, atteignent à peine le commencement de ia cuisse.

On peut voir, dans la collection Erpétologiqque du Muscum de Paris, plusieurs individus appartenant a cette mêrne espèce : ce sont ceux qui ont été recueillis à Coquimbo par le savant botaniste M. Gaudichand.

PLANCHE III.-Fig. 2.

\section{PROCTOTRETE DE WIEGMANN.}

Proctotretus Hicgmanni.

Ce Saurien est d'une taille très-ramassée, sa queue ćtant fort courte, ayant au plus la moitié de la longueur du reste du tronc qui est, en outre, assez large dans la région moyenne.

Le dessus du corps est d'un gris foncé avec des bandes Iongitudinales blanchâtres. Entre ces deux raies dorsales on voit une double série de lunules noires concaves en arrière et cernant des taches blanches. D'autres Iunules semblables, mais plus petites, forment aussi, de chaque còté des flancs, deux séries de taches régulierement espacées et de même étendue. Le dessous du corps est blanc dans toutes ses régions.

L'un des caractères qui est très-propre à faire distinguer cette espèce, c'est que le bord de la lèvre supé- 
rieure, recouvert comme à l'ordinaire de grandes plaques, se trouve surmonté de trois rangées ou bandes d'autres écailles comprises sous la grande platple sousorbitaire. C"est ce yue mpresonte la figure? 2.a) yui est considerablement rrosic ponr faire voir, de côté, toute la partie antérieure du tronc.

La figure (2.e) est destinée aussi à indiquer une particularité de cette espèce dont le dessous de la cuisse oflre, parmi de petites granulations, un amas de plus gros tubercules disposés régulièrement en un quinconce formé de quatre bandes.

Comme la région inférieure vers l'origine du cloaque représentée par la figure (2.d) est celle d'un mâle, on y voit six grands pores à la suite des grandes écailles entuilées, tandis que le pourtour de l'orifice ne porte que de fines granulations.

Il y a également au Muséum de Paris plusieurs individus de cette espere qui ont elé rapportés du Chili par MM. les voyageurs Gay et d'Orbigny. 


\section{ICHTHYOLOGIE}

PAR

H. A. VALENGIENNES.

L'amiral qui a fait, dans la campagne de la Vénus, de très-belles collections zoologiques, n'a rapporté qu'un petit nombre de poissons préparés pendant la relâche aux îles Galapagos. Cê essai d'études Ichthyologiques a été très-heureux, parce qu'il complète ce que les zoologistens anglais ont diga commence sur l'Ichthyologie de cot arehipel pondant l'uxpelition du Bexple. M. Darwin y a reuni plusicurs espoces difrerentes des nôtres. Elles ont été décrites par M. Jenyns avec le talent que l'on pouvaient attendre de cet habile Ichthyologiste, et la plupart ont été figurées. En comparant les espèces mentionnées par cet auteur avec celles que je publie, on peut conclure que la Faune ichthyologique des Galapagos est composée d'espèces qui lui sont particulières, mais qui rappellent plus par leurs affinités les espèces américaines de l'Allantinue que colles the la mer des Indes. 


\section{GENRE SERRAN.}

On sait que le genre des Serrans est l'un des plus nombreux de la classe des poissons: il appartient à la famille des Percoüles à une seule dorsale, et il peut en être donné comme le type le plus caractérisé.

Il est réduit aux espèces qui ont une seule nageoire sur le dos parce que la portion épineuse est unie à la partic soutenue par les rayons articules of branchus. Ces Percoïdes ont des épines à l'opercule, des dentelures au préopercule, des dents en carde assez fortes avec quelques crochets saillants aux deux mâchoires, aux palatins et sur le chevron du vomer.

L'archipel, encore peu exploré des Galapagos, a fourni a l'expedition de l'amiral Inupetit-Thouars deux Serrans nouveaux, dont l'un, voisin du Serramus scriba, a des couleurs vertes et rouges qui sont plus communes chez les Labres que dans le genre que je vais décrire.

Les naturalistes du Beagle ${ }^{1}$ ont trouvé des Serrans aux Galapazos. Cohni que je vais décrire sous le nom de S. Psilucinus est phus volsin du Serramus labriformis, de? M. Jenyns, p. 8, pl. 3, que de son Serramus olfax, ejusả., ib., p. 9, pl. 4. Ces deux espèces sont plus voisines du Mérou que de nos Perect seriba de la Méditerrance. Il n'en est pas de méme de ceux que j'ai fait ligurer. Les miens diffèrent des deux espèces du zoologiste anglais. M. Darwin a donné ces poissons comme étant assez

1 The Zoology of the Poyage of H. M. S. Beagle, by Ch. Darwin. Fishes, ly Rev. Leon. Jenyns. 
communs sur les côtes de l'archipel; je n'ai aucune observation sur ceux que je fais connaitre.

II. Jenyns a décrit dans le voyage du Beagle, pag. 3, pl. 2., un Serrames albo-maculatus qui a les dernicrs rayons de la dorsale très-abaissés comparativement au troisième. Il a aussi observé l'alssence de dents canines; les deux mâchoires ont les dents en velours. Je doute que ce poisson appartienne au genre des Serrans. M. Jenyns semble croire qu'il a plus d'affinité avec nos Centropristes. En joignant au caractère de la première dorsale celui de la forme allongro du museau, de la dentition et des épines de l'opercule et du préopercule, je crois que ce poisson est plutòt intermédiaire entre le genre des Centropristes et celui des Grystes.

\section{PLANGIE I. - Fig. 1.}

\section{LE SERRAN PSITTACIN.}

Serranus Psittacinus, Val.

Il semble que chaque arehipel, chaque mer montrera de nouvelles espèces de Serrans, tant cette forme est abondante dans toutes les eaux du globe. Celui-ci appartient à la division du Serramus scriba, c'est-à-dire que ses mâchoires ne sont pas recouvertes de petites écailles.

Il a la forme ronde et allongée des Serrans des côtes du Brésil, tels que le Serramus irradians : sa hauteur est le cinquième de la longueur totale; sa tête en est plus que le tiers, elle est allongere comme le corps. Livil est de grandeur médiocre et rond; son diamètre est contenu près de cing fois dans la longueur de la têle. Le 
preopercule est arrondi et très-finement dentelé, l'opercule a ses trois épines. La màchoire inféricure lépasse la supérieure. La pectorale est elliptique, ses rayons mitoyens ctant plus loirgs quo les latriaux. La dorsale a sus epines courtes; la caudile est tronquée, un peu concave.

$$
\text { D. } 10 / 12 ; 4.3 / 7 ; \text { C. } 17 ; \text { P } 17 ; \text { V. } 1 / 5 \text {. }
$$

Le bord des écailles est âpre, de médiocre grandeur; il y en a plus de cinquante rangées entre les humérus et la nageoire de la queue.

La couleur générale du corps et des nageoires est un vert assez brillant, le dessus de la tête, le dos, les côtés de la quene, sont rouges. Les deux taches de la quene, et les six autres placées sur les còtés inférieurs du ventre et dela queue ont le centre noir, ce qui donne à ces taches l'apparence de grands ocelles brillants. L'œil est jaune.

Le poisson a $0^{\mathrm{m}}, 21$ de long.

\section{PLANCHE II. - FIg. 1.}

\section{LE SERRAN COLON.}

Serranus colonus, Val.

Ce poisson d'un genre si nombreux el si varié en espèces, est un de ceux qui se rapprochent beaucoup des Serrans, appelés par les habitants de nos Antilles le Colon, ef que nous avons désigné dans notre Ichthyologie ${ }^{1}$ sous la dénomination de Serramus creolus. Il en a les formes générales, el si le ton des couleurs n'est pas le même, leur distribution est tout à fait semblable.

1 Cur., Val., Hist nat Poiss, 1. 11 , page 265. 
La forme du corps est un ovale très-allongé. Le profil du dos est cepeudant un peu plus soutenu que celui du ventre. La hauteur est quatre fois et près de deux tiers dans la longueur totale. La tête est courte, le museau est. assez oftus. Les dentelures du preopercule arrondi sont fines, les épines de l'opercule sont aplaties.

L' ’eil est assez grand; car le diamètre de l'orbite mesure le tiers de la longueur de la tibe, qui est elle-mème comprise quatre fois dans celle du tronc. La bouche est de grandeur moyenne, les branches des màchoires sont nues et sans écailles. Il y a quatre dents coniques dirigées horizontalement en avant et au centre de l'arc supérieur, et en arrière quatre crochets un peu plus forts que les autres dents en carde. Je vois aussi quatre canines à la symphyse de la mandibule. Les dents palatines sont sur une bande tres-étroite, au contraire celles du chevron du vomer forment un petit groupe assez large.

La dorsale est longue, arrondie en arrière; l'anale est plus haute que la portion molle de la dorsale; la caudale est trés-profondément fourchue, car les lobes ne sont que deux fois et un tiers dans la longueur du tronc, ou, ce qui revient au même, trois fois et un tiers rlans la longueur totale. Les pectorales sont longues et pointues, les ventrales sont in peu plus courtes que les antres nageoires paires.

$$
\text { B. } 7 ; \text { D. } 9 / 18 ; \text { A. } 5 / 10 ; \text { C. } 17 ; \text { P. } 19 ; \text { V. } 1 / 5 \text {. }
$$

Les écailles sont petites, finement ciliees; j'en compte cent vingt rangées entre l'ouic et la caudale, et quelIChтHYoLoge. 
ques-unes s'avancent sur le haut de chaque lobe de la nageoire de la quene. Il y en a aussi sur la base de la pectorale. Celles de l'opercule sont semblables à celles du corps, mais celles du préopercule sont plus petites.

Tout le corps de ce poisson est d'un beau jaune de chrome, devenant plus foncé sur le dos, qui prend des teintes un peu vertes. On compte une trentaine do raies obliques sur chaque llanc.

La ligne latérale est tracée parallèlement au dos par le tiers de la hauteur. Elle est formée d'une suite de petits traits moiratres on rembrunis yui répondent ans: tubulures de ses pores efférents.

L'exemplaire que j'ai décrit est long de $0^{\mathrm{m}}, 218$.

La ressemblance que je lui ai trouvé avec le Serran créole m'a fait imaginer le nom de Serramus colonus.

\section{GENRE DIACOPE.}

Le genre des Diacopes est une de ces heureuses creations de M. Cuvier. Il est un de ceux que l'on peut donner conme exemple, pour montrer que l'écude des différences, même les plus légères en apparence, sert souvent à rapprocher les êtres selon leur plus grand degré de ressemblance; c'est ce qui conduit à éablir de bonnes familles naturelles.

La dentition des Diarcopes est celle des Serrans, des Plectropomes, of des Mesoprions; mais l'échancrure an préopercule pour resevoir le tuhercule articulaire de 
l'interopercule, araturise ce groupe grenérique. Il se compose d'espices qui ne sont comulues jusqulà présent. que dans le grand Océan Indien. Presque toutes les Diacopes sont rouges, et les raies dont le corps est orné sont jaunes et dorées; l'espèce dont il va être question est remarquable par sa couleur verte.

M. Darwin n'a pas trouvé cette espèce ni aucune autre de ce genre.

\section{PLANCHE I. - Fig. 2.}

\section{LA DIACOPE VER'TE.}

Diacope viridis, Val.

La Diacope que M. de Fréminville vient de donner au Muséum, offre une variation de couleur très-notable dans un genre aussi naturel que celui des Serrans et des Diacopes, dont le fond de la conleur, et surtout des dernières, est toujours du rouge plus ou moins vermillonné ou carminé. Dans cette espèce, le fond est vert et les raies se détachent par une nuance plus foncée; ces couleurs exceptionnelles sont rares dans un genre naturel. Nous voyons, au contraire, les coulcurs des genres naturels offrir de la constance, soit dans les teintes soit dans les distributions. Ainsi, presque tous les carnassiers de la famille des Félis ont du fauve et une tâche sur l'oreille; le vert, le rouge sont les couleurs les plus constantes chez les Porroquets; les moustaches rouges des pies mâles ef beaucoup d'autres exemples encore viendraient à l'appui de 
cette proposition. Nous retrouvons toutefois dans la Diacope que nous allons faire connaître une distribulion de couleurs semblable à celle de plusieurs autres espèces de ce genre, puisque le corps est rayé longitudinalement. Ln naturaliste (pui s'en tientrait is l'examen rapirle de la disposition des couleurs d'un individu de. coloré, le prendrait pour la Diacope à huit raies (Diacope octo-lineata').

Cette nouvelle espèce a la tête assez longue, elle est comprise trois fois et un tiers dans la longueur totale; la hauteur du corps y est contenue quatro fois et demie. L'wil est grand et le cerste de l'orhite touche presque à la ligne du profil. Le plus long liamedre de l'ail est If longitudinal, et il est du quart de la longueur de la tète. Il est éloigné du bout du museau d'une fois of demie is diametre. Les quatre canines anterieures de l'intermaxillaire sont assez fortes; les dents latérales sont faibles à la mâchoire inférieure, les latérales sont plus grandes que celles de devant. L'échancrure du préopercule et le tubereule du sous-opercule qui s'y articulent sont tris prononcés. Les dentelures du bord montant de l'angle arrondi de l'os sont tris-manifestes. L'opercule est anguleux, mais sans épines. Les premiers rayons épineux sont assez longs, les mous n'ont guère que la moitié de ceux-ci; la caudale est échancrée.

$$
\text { D. } 11 / 14 ; \text { A. } 5 / 9 ; \text { C. } 17 ; \text { P. } 15 ; \text { V. } 1 / 5 \text {. }
$$

Les écailles sont lisses et peu largers; jen compte plu:

8ur., Val., Hist. nal. Poiss, 1. II, p. 418 . 
de cinquante rangeces entre l'ouie et la caudale. La ligne latérale est un peu marquée, à peine visible; la couleur générale est verdàtre, devenant plus foncée sur le dos, et ayant le long des flance, de chaque còté, quatre raies longitudinales droites, vert-fonce; les nageoires sont verdâtres.

La longueur de l'individu est de $0^{\mathrm{m}}, 265$. Cette Diacope est peut-citre rare aux Galapagos, puisqu'elle a échappé aux recherches de M. Darwin.

\section{LES SHECTIQUES.}

Smecticus, Nob.

Nous avons séparé des Grammistes, Percoïdes à dents en velours et à deux dorsales, les Savonniers qui oni une seule nageoire étendue sur le dos. Cette nageoire est remarquable par le petit nombre de rayons épineux qui la soutiennent. L'absence d'épines à l'anale, si rares dans les poissons osseux, constituent les caractères génériques des deux seules espèces connues de l'Atlantique.

L'Archipel des Galapagos a donné un nouveau genre que l'on pourrait facilement confondre avec les Savonniers, si on n'attachait pas assez d'importance au nombre des rayons épineux de la dorsale et à l'absence des épines de l'anale.

En effet, le poisson que j'ai sous les yeux a des dents en velours, des épines à l'angle du préopercule et à l'opercule, caractères qui conviennent aux Rypticus et aux Grammistes. Nais ces derniers on 
deux dorsales; par conséquent le poisson, dont it va être parlé avec détail, ne peut être de ce genre.

Sa forme générale, la briéveté de sa dorsale conchée sur le dos, la forme de l'anale, la dentition l'appelleraient anpress des liypticus; mais ceux-ci n'ont que trois rayons à la dorsale et en manquent à l'anale, tandis que la nouvelle espèce en a dix à la dorsale et deux à l'anale. Je n'avais pas cru d'abord, à cause de cette difierence dans les rayons des deux nagreoires verticales, faire un genre de notre nouveau poisson, parce que tout le reste du poisson me paraissait le placer avec les Savomniers de l'Atlantique. Aussi c'est sous ce nom générique (qu’il a paru dans l'atlas, pl. II, fig. 2; mais en l'étudiant avec plus de soin, je trouve dans ces formes, d'un poisson de l'Mllanlique, phusicurs caracteres empruntés aux Grammistes du grand océan Indien. Il a, comme eux, deux épines à l'anale et une dorsale soutenue par plusieurs rayons ipineux; mais il n'a, comme les Savonniers, qu'une seule dorsale. C'est donc un assemblage des caractères des deux genres. L'importance de ces caractères m'a déterminé à considérer cette nouvelle espèce comme un genre nouveau, et elle servira à fixer d'une manière plus étroite et plus philosophirgue les caracteres les Rypticus, qui rentrent dans les formes générales des Percoïdes à une seule dorsale et à dents en velours.

Le nom de Shecticus que je donne il ce nouveau genre rappelle lestihites des Saromniers aver lespere que je vais décrire sous le nom suivant: 
PLANCHE II. - Fig. 2.

SMECTYUE AUX DEUX COULEURS.

Smecticus bicolor. Val.

(Ryplicus bicolor, pl. 11, lig. 2.)

Celte espèce a le corps allongé. La ligne du profil est assez soutenue sur la nuque, puis elle s'abaisse et se rend assez droit à la queue, celle du ventre est plus horizontale. La hauteur est a peu de chose près le cinquième de la longueur totale; la queue n'a pas tout i fait moitié de la hauteur prise à la nuque.

La tête est trois fois et quatre cinquièmes dans la longueur totale; le preopercule est arrondi, et le limber est armé vers le haut d'une épine plate, triangulaire, assez forte. L'opercule a deux pointes plates et plus petites que la précédiente; l'ail est dóigné du bout du musean d'une fois son diamètre, lequel est le cinquième de la longueur de la tête.

La bouche est peu fendue; les dents sont igales et en cardes fines, sur une bande ctroite aux deux mâchoires, aux palatins et sur le chevron du vomer; la langue est libre, étroite, pointue et lisse.

La dorsale s'élève à peu près au tiers de la longueur ; assez au delà de l'insertion de la pectorale; elle a dix rayons épineux, assez bas, et dont les trois premiers sont cependant un peu plus haut que les suivants; la dorsale, l'anale, la caudale et les pectorales sont arrondis, les ventrales sont petites.
B. 7 ; 1). 10/21;
A. $2 / 15 ;$ C. $17 ;$ P. $15 ;$ V. $1 / 5$. 
Les écailles sont très-petites, j'en compte cent vingt rangées au moins entre l'ouïe et la caudale.

La ligne latérale remonte en se courbant vers le dos, puis elle s'infléchit lentement, de manière ì se terminer par le milieu de la hauteur de la queue, tandis (fu'clle s'est maintenue sur le trone par le cinquieme ou le quart de la hautenr.

Ce poisson est d'un beau rouge vermillon, marbré plus clair, avec toutes les nageoires noires.

L'exemplaire qui est déposé dans les collections nationales du Muséum est long de $0^{\mathrm{m}}, 230$.

\section{GENRE CIRRHITE.}

Le genre des Cirrhites ne se compose jusqu'it présent que d'un petit nombre d'especes, presque toutes remarquables par la vivacité de leur couleur, mais les individus restent dans de petites dimensions. Le Cirrhite que je vais faire connaitre, sans itre encore très-grand, atteint à la taille d'une Carpe ordinaire. Pour ceux qui s'en tiendraient sans aucun examen à la diagnose du genre Cirrhite, je ne m'étonnerais pas de voir placer le poisson que je vais décrire dans un genre distinct des Cirrhites. Ceux-ci ont, entre autres caractères, le bord montant du préopereule dentelé; or, sur l'individu que j'ai sous les yeux, je ne puis apercevoir aucune dentelure le long du bord de cet os. Mais il fant faire attention que l'individu dont il s'agit a le double au moins de grandeur des autres Cirrhites connus, et il faut alors se rappeler que dans beancoup de Percoïdes, et no- 
tamment parmi les Serrans, nous voyons les dentelures s'évanouir avec l'âge du poisson. Notons aussi que dans le Cirrhites pantherinus ${ }^{\text {, }}$, les dentelures sont d'une finesse excessive, et qu'on ne les aperçoit qu'à l'aide d'une forte loupe. L'exception ou l'absence de dentelures au prépercule doit derenir un caractire peu important quand nous trouvons que ce poisson offre tous les autres caractieres du grenre aucquel nous le rapportons. Nous observons, en première ligne, les rayons simples de la pectorale, et les dents vomeriennes; puis la disposition des dents des mâchoires, et l'ensemble du facies ne peuvent laisser aucun doute.

\section{PLANCHE III.- Fig. 1.}

\section{CIRRHITE RIVULÉ.}

Cirrhites rivulatus. Val.

La nouvelle espèce de Cirrhites que je vais décrire est la plus grande et une des plus ornées dans ce genre.

Son corps épais est assez élevé à la nuque et au devant de la dorsale; puis il diminue assez promptement de hauteur, de façon que celle du tronçon de la queue derrière la dorsale n'est plus que du tiers de celle du tronc a la reigion des pectorales. Cette hauteur est comprise trois fois et cing sixièmes dans la longueur totale. Celle de la tête éggale la hauteur du tronc. Le front est crensé en une sorte de gonttiere entre les deux yeux. Ine crèle bifurquée arrite cette gouttiere, et forme

'Cuv., Val., Hist. nat. Poiss, t. III, P. Fo. 
une coulisse dans laquelle jouent les pédicules des intermaxillaires : l'œil est placé très-haut sur la joue; son orbite entame fortement la ligne du profil. Son diametre fait le quart de la hauteur, of plus du sixième. de la longueur de la tête. Il est éloigné du bout du museau de deux fois la longueur de son diamètre.

A peu de distance de l'wil sont les doux ouvertures de la narine. La postérieure est petite et rapprochée du cercle de l'orbite; la premicre, plus en avant, est munie d'une forte caroncule charnue. Le sous-orbitaire, étroit et oblong, est entièrement caché sous une peau épaisse. Le préopercule a le bord arrondi en demi-cercle, et recouvert aussi sur tout le limbe par un cuir très-épais. Le reste de la jone a de très-petites écailles. L'opercule a une pointe plate et mousse, son bord membraneux est large et anguleux. Le sous-operculc est petit, et l'interopercule est grand et nu. La gueule est large et bien fendue. La màchoire supérieure a quatre fortes canines, puis un rang de dents coniques le long du bord, et derrière, une bande de dents en velours. A l'inférieure on trouve un groupe de dents en velours, et une petite canine sur le rang externe; puis sur les côtés deux canines plus grosses, ensuite une quatrième grande, forte et conique, suivie d'une plus courte, et de plusieur's plus petites dans l'angle. Le groupe de dents vomériennes est très-sensible. La dorsale antéricure est basse, soutenue par de fortes épines; une échancrure bien prononcée la sépare de la seconde qui est beaucoup plus haute. La caudale est conpée carrément; l'anale est longue et étroite. La pectorale grande a ses rayons 
branchus plus longsque les simples. On en compte sept de ces derniers. De très-petites écailles montent sur la membrane des nageoires impaires, principalement sur la dorsale.

$$
\text { D. } 10 / 11 ; A .2 / 6 ; \text { C. } 17 ; \text { P. } 7-7 ; \text { V. } 1 / 5 \text {. }
$$

Le tronc est recouvert d'écailles solides, et beaucoup plus grosses que celles du préopercule. Eilles sont trèsfinement striées et chagrinées. J'en trouve environ quarante-cinc entre l'onie et la caudale. La ligne latérale est droite et par le tiers de la hauteur.

Ce poisson a le corps vert-olive plus ou moins foncé. sur ce fond on roit ales taches plus claires, bordées d'olive foncé, et liserées de beau bleu de cobalt. Ces márbrures et les rivulations qui les bordent forment cing grandes et larges bandes sur le trone. Quatre bandes olive rembruni, et liserées de bleu de cobalt traversent les joues, une sur le sous-orbitaire, deux sur le préopercule, et la quatrième à l'angle de l'opercule. Denx autres taches oblongues entoures d'une ellipse bleue, sont à la base de la pectorale; les nageoires olivatres ont des rivulations blenes. Elles forment sur la caudale une sorte de réseau hexagonal qui doit ètre fort agréable à voir pendant la vie de ce beau poisson.

La longueur de l'individu donné an Muséum d'histoire naturelle, par M. de Fréminville, est de $0^{\text {ma }}$, 4.5. Je ne vois pas de Cirrhite cité dans le travail de II. Jenyns. Est-ce une preuve que ce poisson soit rare aux Galapagos? 


\section{GENRE SCORPENE.}

La famille des Percoïdes à joues cuirassées par le sous-orbitaire articulé avec le préopercule, a donné, dans les mers des Galapagos, plusieurs espèces intéressantes pour l'Ichthyologie. M. Darwin y a trouvé un Prionote nouveau, que M. Jenyns a fait connaître sous le nom de Prionotus Miles.

Le genre des Scorpènes, limité par M. Cuvier aux espèces qui ont la tête grosse armée d'épines, et dont la dentition se developpe sur les maxillaires, les palatins et le chevron du vomer, a aussi fourni au mème naturaliste une espèce qui a pris rang, en Ichthyologie, sous le nom de Srorpana histrio. Elle est différente de celle que j'ai à décrire.

Les Scorpènes sont propres aux mers équatoriales ou a celles qui baignent les parties chaudes des zones tempérées: nous avons dimontré dans notre Ichthyologie que les poissons des mers du nord de l'Europe rangés, avant nous, parmi les Scorpènes appartiennent à des genres dillérents. Lne seule des Scorpènes d'Europe, le Scorpana Porcus se montre à de rares intervales dans la Manche. L'Allantique européen, et le grand bassin de cet Océan, compris entre l'Afrique et l'Amérique, en nourrit moitié moins d'espèces que la mer de l'Inde. Puisque les espieres sont plus communes dans le grand Océan Indien, il n'est pas surprenant que M. I)arwin et MI. l'amiral Dupetit-Thouars aient donné I'oreasion d'en faire enonaitreanx fehthyologisteste non- 
i elles, pendant la reliche qu'ils ont faite aux Galapagos.

A cause des conleurs rouges dont les côtés de la tête sont colorés, ce qui rend les joues du poisson comme fardées, je propose de désigner cette nouvelle espèce.

\section{PLANCHE III. - Fig. 2.}

\section{LA SCORPENE FARDÉE.}

Scorpona fucata, Val.

Cette Scorpene rappelle par ses marimures et par ses écailles notre Scorpann scrofa de la Véditerranée : mais elle n'a pas comme elle le corps couvert de lambeaux cutanés. Elle a les crètes des joucs et de la tìte plus prononcées. Le trone, gros et trapu, est à fueu près rond à la région pectorale, car le diamitre transtersal mesure les six septièmes de la hauteur, l'épaisseur de la queue est encore moitié de la hauteur. La tìte est grosse et longue comme dans toutes les espèces de ce genre; la longueur est à peu de chose près le tiers du corps entier. Les yeux sont gros et saillants. Le diamètre est compris trois fois et quatre cinquiemes dans la longueur de la tête. L'intervalle qui les sépare est égale à la moitié du diamètre. Il est profonclément creusé à cause de la hauteur des crètes surcillières. Chacune porte en avant une très-forte épine, en arrière deux très-courtes. Une petite carène frontale peu saillante s'avance jusqu'an delà de la narine, et elle est armée d'une forte épine. Derrière les épines postérieures de la crète de l’orbite, on voit une épine mastoüdienue réunie à celle du côté opposé par une crête transversale, et au delà il existe 
deus autres épines dont la derniere est la plus forte: elles sont reunies far une crête transversale occipitale concave, et moins élevée que celle qui précède. Je vois derrière l'neil deux petites épines comprimées, tranchantes, mais peu élevées, dont la plus grosse est au-

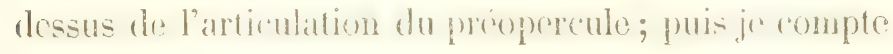
denx épines oblongues sur le suscapulaire, et enfin une épine termine le haut du scapulaire. L'intervalle entre toutes ces petites épines est semé de granulations osseuses quelquefois hérissées.

Le préopercule arrondi porte trois fortes épines à son angle, et deux sous le limbe horizontal.

La crête du premier sous-orbitaire qui traverse el cuirasse la joue, se rend à l'épine supérieure; le bord inférieur du sous-orbitaire est armé de quatre cipines.

L'opercule a deux fortes carènes divergentes et terminces dhacune par une pointe aiguei qui depasse le bord de l'os. Les dents des mâchoires sont en velours assez rare; celles des palatins et des chevrons du vomer sont en cardes; ces dernières sont un peu plus fortes.

La dorsale avance vers l'occiput de manière que le quatrieme layon épineux correspond à l'angle de l'opercule. La portion molle n'occupe en Iongueur que la moitié de la portion épineuse. Les rayons épineux de l'anale sont très-forts; la caudale est tronquée. La pectorale druasse un peu le tiers do la longueur du trone. Les ventrales sont petites.

B. 7; D. $12 / 10$; A. 3/5; C. 15 ; P. 19 , dont 9 simples; V. $1 / 5$.

Les écailles sont asscz grandes et lisses; j'en compte 
quarante-deux rangées entre l'onie et la caudale. La ligne latérale est presque droite.

Le fond général de la couleur est un vert d'ean plus clair sous le ventre, et plus vif et plus foncé sur la tête; les joues sont colorées d'un beau rouge marbré plus foncé sur le préopercule et sur les crêtes. Les taches du dos et des flanes sont lie de vin. La dorsale a le fond vert, un peu citrin, avec des taches lie de vin claires : le bord de la portion molle est rosé. La caudale a la base verte, et le bord libre rosé assez vif; je vois trois rangées de points rouges sur la base de la pectorale qui est verte avec le bord rosé.

L'anale et les ventrales vertes n'ont aucunes taches.

La longueur de l'individu est de 0, 223.

\section{GENRE MYRIPRISTIS.}

Parmi les Percoïdes nous avons signalé un certain nombre de poissons qui ont plus de cin'j rayons aux ventrales. Il est assez curieux de voir comment la nature offre tout d'un coup une déviation à un nombre aussi géné ralement adopte par elle pour presque tous les Acanthoptérygiens. On aurait pu croire ì l'établissement d'une loi qui n’anrail pas manqué d'itre soutenue par une comparaison naturelle. On considere les nageoires paires. comme los représentants des quatre menbres des vertébrés. Les pectorales correspondent aux membres antérieurs, ct les ventrales anx postérieurs. Trouver dans: la généralité des espèces cinq rayons articulés aux nageoires ventrales, donnait lien do croire que ce nombre 
variable pour les pectorales rappelait an moins celus des doigts des membres du plus grand nombre des vertébrés; mais une exception se présente dans les Holocentres, les Myripristis, les Beryx, dont le nombre est sourent de sept, of dipasise quelyuefois pour atteindre à celui de dix.

Les Myripristis sont, à l'exception d'un seul, originaires du grand Océan. L'Atlantique en nourrit un connu dans les Antilles sous le nom de Frère Jacques; c'est notre Myripristis Jacobus.

Celui des Galapagros a luaucoup de rapport avec cette espèce; je lui trouve un 'aractere saillant et distinctif dans ses mâchoires lisses; c'est ce qui me le fait appeler :

PLANCHE IV. - Fig. 1.

\section{I.E MYRTPRISTIS AUX MACHOIRES LISSES ov MYRIPRISTIS LEIGNATHE}

\section{Myripristis Leignathos}

Ce Myripristis est cigalement voisin de celui qui a été trouvé au port Praslin de la Nouvelle-Irlande, et que nous avons nommé M. Parvidens, à cause de la petitesse de ses dents. Celui dont nous nous occupons a les dents au moins aussi petites, et il offre comme lui, le caractere de manquer de dentelures à l'ancrle du maxillaire; mais il a l'intervalle entre les yeux plus étroit, l'oeil plus grand, et les nombres un peu différents.

Ce poisson a, comme la plupart de ses congénères, le corps en ovale régulier, jusqu'i la naissance du tronşon 
do la quene. La hauteur fait pres du tions de ha lonsucur totale. L'épaisscur n'est gucre que moitié de la hanteur.

La tête est courte, sa longueur est comprise trois fois et trois quarts dans la longueur totale. L'coil est granr ; cependant son diamètre est lan peu moindre de la moitié de la longueur de la têle. L'orbite n'entame pas la ligne du profil, mais il la touche.

L'intervalle entre les deux yeux égale lo quart de la longueur de la tête. Les deux ouvertures de la narine sont petites, rapprochées l'une de l'autre et de l'orbite, la postérieure a une fente oblongue plus grande que l'antérienre. Les palmures du crâne sont peu profondément ciselées, et précédées de deux arêtes divergentes entre les yeux. Sur le devant du crine est une échancrure pour recevoir les pédicules les iniermaxillaires. Le sous-orbitaire est étroit, et a ses deux bords faiblement dentefós. Le próperoule, l'opiercule, et l'interopercule sont aussi finement denteles: le hord du sous-opercule est lisse, et n'a aucme denteinre ni épine.

L'intermaxillaire a le bord étroit, le maxillaire a quelques stries très-fimes, son angle est arrondi, il n'a aucune épine ni dentelure. L'os de l'épaule est également lisse. La dorsale a des rayons grêles, dont le second, double du premier, est compris deux fois et demie dans la hauteur du tronc mesuré sous hui; les rayons commencent à diminuer à partir du cinquième; le remier est très-petit, et presque entiorement caché dans la rainure de la dorsale. Le premier rayon de lia 
seconde dorsale ast me épine assez forte; celte na. gooire est assez nettement séparée de la précédente. La ¿audale est peu échancrée; les épines de l’anale sont rourtes.

D. $111 / 12 ;$ A. $4 / 15 ;$ C. $6-17-5 ;$ P. $15 ;$ V. $1 / 7$.

Les écailles ont le bord strić et même dentelé, j’en compte trente-huit rangées cntre l'ouïe et le caudale. La ligne latúrale suit parallèlement à la courbure par le fuart supérjenr de la hauteur.

Iat coulcur est un beau rouge vermillon à reflets dorés, qui rendent le poisson très-brillant.

La longuem de l'individu que je décris est de $00^{\mathrm{m}}, 16 \%$.

\section{DU GENRE DOYDINODOS.}

La lamille des Sciénoïdes est représentée aux Galapagros par des espèces dont nous ne comnaissons encole qu'un très-petit nombre. M. Jenyns n'en a eu que trois à faire comnaitre, et la collection de l'amiral n'en fournit pas une seule. De ces trois espèces, l'une vient augmenter un genre qui compte déjà beaucoup d'espèces, celui des Pristipomes. Mon savant collègue l'a nommé Pristipoma cantharimam, parce gue ses teintes rappellent celles du Cantharus griseus de Ia Manche; les formes de ce Pristipome sont celles des cspèces de l'Atlanlique, et principalement du Pristipoma rubrum, it cause du peu de force du second rayon ápinenx de l'anale. II atependant le maseau un peu phus aromdi.

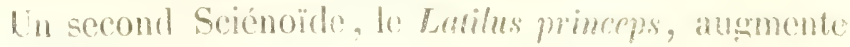


"ce greme peu nombreux, dont nous avons décril trois es. preces, Lat. argentatus ${ }^{4}$, Lat. doliutus ${ }^{2}$ el Lat. clrysops ${ }^{2}$; rar je ne parle pas ici de mon Latilus jugularis qui offre une légère différence avec celles-ci.

Je trouve encore dans le travail de M. Jenyns une intéressante découverte due à M. Darwin, dont le zoologiste dir Beagle a fait le genre Prionodes. II faut toute la juste confiance que l'on doit ajonter aux travaux de mon célèbre confrère, pour ne pas être plus étonné de la nouvelle conbinaison de caracties zooloniques que la nature a faite dans ce poisson. On pourrait dire, si l'on osait s'exprimer ainsi, que le Prionode est un Serran auquel la nature a enlevé les dents du palais. Les couleurs de la senle espèce vue par M. Jenyus sont également très-semblables à celles de nos Serrans communs.

Je n'ai trouvé aucune de ces espèces intéressantes, mais les naturalistes de la Vénus ont eu le bonheur de prendre deux autres poissons de la fanille des Squammipennes dont M. Darwin ne me parait avoir trouvé aucun représentant. L'in d'eux constitue dans les Squammipennes un genre nouveau des plus remarquables qui vient s'ajouter à la petite tribu des Squammipennes à flents tranchantes. Calles de l'esperce sur lapuelle nous établissons le genre Doydrodon sont différentes des dents des Piméleptreses of des Dipterodons, et à cause rle leur grand nombre et de leur finesse, 'clles fixen les rapports qui lient les deux genres Piméleptères et

1 Cuv., Val., List. nat. Poiss, t. Y, pag. 569 et 5z1.

"Eorumdem, ibid., 1. IX. Suppliment au volume V. mag. $4 g^{6}$. 
Diphendous de la sande fanille des siquammipenner. Les deux genres nommés plus haut me paraissent y représenter les Perches, les Bars, les Apogons et autres genres qui forment la premiere famille des Percoïdes à deux dorsales; je crois donc que la tribu dénommée d'après les dents dans notre Histoire des Poissons, 1. VIII, p. 刃̋z, serait caractérisée plas philosophiquement par le nom de Squammipennes à denx dorsales. Elle deviendrait sous ce nom le groupe parallèle a notre Perche et aux espèces voisines. Le genre Doydixodon devient le lien qui unit cette tribu à celle des autres Squammipennes à une senle dorsale; car la nageoire dorsale du poisson, dont il va être traité dans cet article, est unique, mais les derniers rayons heaucoup plus bas que ceux yui les precedent, laissent aisément croire à la présence de deux nageoires. Cependant on acquiert bientòt la prenve de leur connexite et de leur continuité en enlevant les écailles étendues repuis le troisieme rayon jusque sur la fin de la nageoire. Toutes les autres nageoires sont écailleuses, à la maniere des Chatodons, des Holacanthes et de tous les antres groupes génériques de la famille. Il n'y a done aucun doute possible, par l'examen de ce caractère, sur la place à assigner à notre nouveau genre.

Les dents, quoique difierentes, sont cependant faites d'après le plan de celui des Piméleptères, mais en marchant vers la figure des dents des Chrofodons. Elles sont en effet implantées sur plusieurs rangs. Elles paraissent d'autant plus longues sur le poisson desséché, que l'on en voit toute la racine, à cause du retrait des 
farties molles de la levre sur laquelle elies sont évidenment mobiles. Cette racine forme un talon dirigó presque à angle droit sous la couronne, comme dans le Piméleptère, mais elle est rejetée ou reculée en arrière; la dent est donc un peu coudée. La couronne est en forme de cuilleron. Les germes se développent au fond d'une gouttiere de l'os. Ces dents trè-nombreuses descendent après leur croissance, et sont prises par la lève avec layuelle elles resient mobiles; mais comme elles sont chassées par la succession incessante des nouveaux germes, elles fuissent par se souter sur plusieurs rangs sur le corps de l'os en dedans et hors de la lèvre, of elles font ainsi une seconde rangée interne que le retrait des parties molles rend encore plus sensible.

L'étude de cette curieuse dlentition fournit une nouvelle preuve de ce que j'ai souvent vu dans l'histoire de l'Ichthyologie. La nature, dans sa force créatrice, si puissante el si active, trouve toujours le moven, en employant les mêmes éléments dans dés conditions analogues, d'arriver à un résultat qui paraì trés-différent. Si l'os de la mâchoire ne contenait pas le germe des dents dans cette rainure, si la levre qui recouvre l'os ne venail pas les prendre, mais laissait ces germes arlhérer sur la face extérienre de l'os; nous aurions dans cette famille une mâchoire de Scare ou de Tétrodlon. La nature a employé le morle de formation et de suecessıon des dents de ces derniers, en conservant au produit le caractère des dents longues, grêles, serrées les unes contre les autres de la fimille des Squammipennes. fes chudes détallées des différenees établies par la nature font comprendre 
parfaitement la structure des màchoires des Scares ou des Tétrodons tels que Cuvier l'a expliquée.

Je n'ai pu compter que cinq rayons à la membrane branchiostége, les Pimcleptères en ont sept, dont quatre seules sont faciles à trouver; il faut pour observer les autres une dissertion altentive. J'ai cru que les Dipterodons n'en ont que six; on ne doit pas s'étonner que ce nombre vienne a varier flans un autre genre. Mais comme? je n’ai examiné qu'um exemplaire desseche, il ne serait fas impossible que des naturalistes plus heureusement placés que moi ne drouvassent un nombre plus grand de rayons sur un individu conservé dans l'alcool, et sur lequel on pourrait compter les xayons à l'aide du scalpel.

Il résulte donc de ces observations que les Doydixodous sont des Srfuammipennes a une seule dorsale fortement échancrée comme divisée aurlevant de la portion molle; que lus dents mobilessur les lives, at implanties sur plusieurs rangs, ont la couromne dilatée, arrondie en dehors et creusée en cuilleron en arrière; le palais est lisse et les opercules n'ont ni épines ni dentelures.

La membrane branchiostége est soutenue par cinc rayons.

Je ne connais encore qu'une seule espèce de ce genre remarquable, que je me fais un plaisir de dédier à M. de Freminville, officier de marine fort instruit, qui a rendu de grands services aux sciences naturelles sous la direction scientifique qu'il a recue de M. Alexandre Brongniart et aux soins colairés duguel les coller- 
fions du Muséum d'histoire naturelle sont redevables ales produits ichthyologiques de l'expédition de la Vémus.

\section{LE DOYDIXODON DE FREMINVILLE.}

Doydixodon Freminvillii, Val., Poiss. Vénus, pl. 5.

La régularité du profil, la grosseur et la largeur de la léte, rappelle la forme générale de nos larpess, en mème temps que la forme horizontale de la bouche, l'épaisseur des lèvres et des mâchoires et leur mouvement. rattacheraient cette singulière espèce au grroupe des Muges.

Ce poisson a des formes trapues. La hauteur di tronc est trois fois et un tiers dans la longueur totale; l'épaisseur mesure les deux tier's de la havteur; la lête est courte et large, le museau tròs-gros et obtus; l'échancrure de l'occiput, marquée par le sinus rentrant de l'attache des muscles du dos, atteint au moins au milieu de l'œil; l'intervalle qui sépare les deux yeux égale deux fois et demi leur diamètre. Il n'y a que deux diamètres au devant de l'œil, et encore cela dépend-il de l'épaisseur de la lèvre. L'orbite est tout à fait sur le haut de la joue; le diamètre de l'orbite est trois fois et un tiers dans la longueur de la tête; le sousorbitaire, cachésous une peau tris-ipaisse, estau derant, mais peu an dessous l'œil; son bord postérieur est ichancré en arrière; le préoperrule, légèrement courbé, est haut ef étroit; le limbe et la joue sont entierement fouverts d'ecailles, landis que les trois andes pioces de 
l’appareil operculaire en sont totalement dépourvues; eiles n'ont ni épinos ni dentelures; les lèves sont d'une épaisseur remarquable, ainsi que les intermaxillaires et la màchoire inférieure. Les premiers ont une branche montante assez prononcée pour rendre la bouche in peu protractile, l'os lui-mème est creux, et contient dans sa racine, reconverte par la levre, les germes nombreux des dents tres-singulieres de ee poisson. Il no m'est pas difficile d'en compter sept à huit rangées. Les dents mobiles dans l'épaisseur de la lèvre descendent au fur et à mesure qu'elles sont poussées par les rangées supéricures, et alors elles se soudent avec te corps aplati et élargi de l'intermaxillaire. Cet os est formé d'une lame arquée, étroite à l'angle de la bouche, montant en s'élargissant de manière à présenter une plaque triangulaire, dont le bord interne se courbe pour compléter l'axe dentaire en se rémissant à l'intermaxillaire opposé. Le bord externe de l'os se porte en arrière et forme une voùte à parois minces pour constituer la rainure ou sont les germes dont j’ai parlé plus haut, et ine recouvert par les lères épaisses qui sertissent les premières rangées de dents mobiles, avant qu'elles ne descendent se souder sur le corps de l'intermaxillaire. Le naxillaire mince est, aplati en lames cachées derrière la levre, sous lo bord libre du sous-orbitaire, mais il se plie en se contournant sur lui-même en formant une large palette que l'on voit a l'angle de la bouche le long des branches de la mùchoire inferieure. Celles-ci sont faites comme l'intermaxillaire, c'est-a-dire que leur branche s'elargit en avant el cn dedans on unc palette triangu- 
laire qui vient se poser sur la plaque supérieure de l'inturmaxillaire quand la bouche est former. Elle porte de nòme les dents fixées à l'os, mais en dehors l'os se creuse en une large gontlière recouverte par une lèvre "maisse garnic de dents. La description deces différents os et leur mouvement prouvent que j'ai eu raison de les comparer à coux de la bouche des Muges; ils en diff'irent cependant beaucoup par la dentition. Le palais est lisse et sans dents; l'ethmoïde forme à l'extrémité au devant du cherron du romer deux grosses eminences boursoutlées; les palatins sont dilargis en deux lames latérales et presque verticales.

Les dents ont wne forme très-particulière. Portée sur un très-long talon, la couroune ressemble à un petit cuilleron, pl. š-C., redressé et faisant un angle avec la tige radicale. Elles sont serres par lignes ohliques variant de six à quatre même à deux seulement près de l'angle externe; ces dents gencivales sont stivies d'autres semblables, mais beancoup plus petites, qui sont fixes sur le corps de l'os. Sur les côtés de chaque màchoire, il n'y a qu'un seul rang de petites dents de mème forme; la dessiccation et le retrait de la lèvre paraissent distribucr ces dents sur deux rangées distinctes, ainsi que cela est représenté pl. "̈, A-B.

La dorsale épineuse est beaucoup plus basse que la portion molle, ce qui peut faire croire ì deux dorsales; mais comme les écailles remontent sur la base de la partie postérieure, on retrouve ici ce que présente un grand nombre de Squammipennes. Le premier rayon repond an second fiers de la pectorale; ces espèces 
sont courtes et fortes. L'angle de parties molles est arrondi, le postérieur est aigu, la caudale et l'anale sonı échancrées; la pectorale, pointue à son extrémité, a le bord inférieur arrondi.

$$
\text { B. } 5 \text {; D. } 12 / 20 ; \Lambda .5 / 15 \text {; C. } 25 ; \text { P. } 18 ; \text { V. } 1 / 5 \text {. }
$$

Je compte de quarante à quarante-cinq rangées

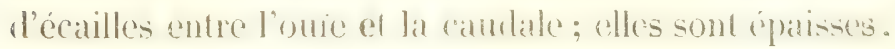
solides, plus hautes que larges, à surface sculptée ou

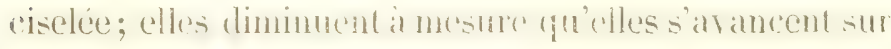
les nageoires, el sur la mupue of lessoupulaires; celles fles préopercules sont aussi très-petites; le reste de la tète en est tout à fait dépourvu.

La couleur est un vert rembruni et presque noirâtre sur le dos et le sommet de la tête. Les nageoires et surtout les portions épineuses de la dorsale sont claires.

La longueur de l'individn est de $0^{\text {ni }}, 4.30$.

\section{GENRE IIOLACANTIIE.}

Le second Squammipenne rapporté par la Vénus ap. partient à un genre bien déterminé par Lacépède, mais il est d'une espèce nouvelle, que M. Darwin n'a pas trouvée.

Le genre des Holacanthes, représenté dans l'Atlantique par deux espèces seulement (Hot. ciliaris ot Hol. tricolor, Lac.), est nombreux dans tout le grand Océan indien. L'espece nouvelle que j’ai à fiure conmaitre ressemble le plus par la distribulion des couleurs an Che uodon asfur de Forskal, originaire to la mer liouge. 
Elle en diffère par les teintes des couleurs et par un caractère plus important. Le préopercule du Ilol. asfur n'a pas de dentelures sensibles; elles sont au contraire tris-prononcées le long du bord montant du préopercule de notre poisson.

Comme le mot asfur, que les Lrabes de Lohaja donnent a cet Holacanthe, signitie Moinean, j’ai voulu rapueler, par l'épithète de l'espèce nouvelle, son affinité avec l'Holacanthe de la mer Rouge.

\section{PLANCHE VI.}

LHOLACANTHE MOINEAU.

Holacunthus passer, Val.

Cette espèce nouvelle d'Holacanthe est, comme je viens de le dire, voisine de l'Iolactmthus asfur du Forskal.

Son corps est comprimé, et le profil du tronc en ovale régulier assez allongé. La hauteur est deux fois et demie dans la longueur totale; la tète est courte, le museau fait une courte saillie. La bouche est petite, peu fendue, ses dents longues et fines sont serrées les unes contre les autres. L'œil est au haut de la joure. Il est écarté de l'autre d'une fois le diamètre qui mesure un peu du tiers de la longueur de la tête; celle-ci est comprise cing fois dans la longueur totale du corps. Le sous-orbitaire, aussi haut que l'œil est larye, porte quatre épines courtes, mais fortes sur le bord libre. Le préopercule a son bord obligue fortement dentele. II domme 
de son angle un long et grand aiguillon strié qui atteint jusqu'à la pectorale, et au-devant de lui il y a sur le bord horizontal une épine courte et forie. L'interopercule porte trois fortes pointes aiguës; les deux autres os cachés sous les écailles n’ont aucunes épines ni dentelures.

La dorsale est étendue tout le long du dos, en partie cachée à partir du troisième rayon sous les écailles nombreuses qui la revetent. L'angre de la portion molle se prolonge en un lobe pointu, très-aigu, ce qui rend, ̀̀ cause de la brièveté de l'angle postérieur, le bord de cette natreoire tris-fortemont ichaneri. L'anale ressemble à la dorsale, mais elle est plus courte; la caudale est tronquée, la pectorale secondaire arrondie en dessous, les veutrales sont un peu plus longues.

B. $7 ;$ D. $14 / 18 ; A .5 / 18 ; C .17 ;$ P. $17 ;$ V. $1 / 5$.

Ce poisson est comvert d'érailles épaisses, solides, et tris-profontement striés, ce qui rend sa surface trèsrude. Je compte soixante-quinze à quatre-vingts rangées d'écailles entre l'ouïe et la caudale qui est comme les autres nageoires, et toute la tête couverte d'écailles semblables à celles du corps, mais beaucoup plus petites. La ligne latérale suit la courbure du dos par le quart de la hauteur.

La couleur est un brun uniforme traversé par une large bandelette argentée descendant sur le tronc ¿ la hauteur de la sixième épine dorsale. La partie épineuse de cette nageoire, la caudale, les pectorales of les ventrales, sont d'un jaune soufre. La dorsale 
et l'anale sont bordées d'un trait jaune liséré de brun.

La longueur de l'exemplaire est de $0^{\mathrm{m}}, 2650$.

\section{DU GENRE DAURADE.}

Le Sparoüde que j’ai à faire connaìtre dans cet article pourrament tout aussi bien ètre placés parmi les Pagres que dans le genre des Chrysophrys. C'est une de ces espèces intermédiaires difliciles à placer, parce qu'elles ont des caractères qui tiennent de plusieurs groupes. Ce Sparoüde a en effet, comme les Pagres, des dents molaires sur deux rangs; comme les Pagcls, des dents en cardes assez funes derriore les incisives, et il a, comme les Daurades, des molaires rondes ou ovales, à couronne tellement large, que jai trouvé dans le développement de ce caractore un trait plus saillant des Chrysophrys que de tout autre genre.

Je concerrais cependant que des naturalistes vinssent critiquer la place que j’ai assignée à ce poisson; cependant je rois avec plaisir que je tromve le mème sentiment dans M. Jenyns. M. Darwin a rapporté cette mème Daurade. Elle est décrite est figurée, dans la zoologie du Beayle ${ }^{4}$, sous le nom que je m’empresse ici d'adopter, afin de ne pas augmenter la liste des synonymes, déjà trop nombreux. M. Jenyns n'a eu, comme moi, que cette seule espèce de la famille des Sparoïdes.

Jenyus, Fish. of Beagle, pag. 56, pl. 12. 
PLANCHE IV. - Fir. 2.

\section{A DAUHADE CHAYOPTBRE.}

Chrysophrys Taurina, Jen. ${ }^{1}$.

Ce poisson a le profil de nos Spares à museau tron. qué, la courbe du dos arquée et très-soutenue vers la nuque, la ligne du ventre presque droite.

La hauteur est comprise trois fois et quatre cinquièmes dans la longueur totale. La tète, à peu près aussi courte qu'elle est haute, est aussi comprise quatre fois et un tiers dans la longueur totale. L'oil est grand sur le haut de la joue. Le sous-orbitaire forme sur le devant de la joue $u$ large trapèze recouvert par une peau adipeuse. Le préopercule est étroit et couvert de cinq à siz rangées d'écailles. Tout l'opercule, le sous-opercule et l'inter-

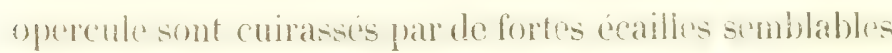
à celles du tronc. Les deux premiers de ces os sont confondus sous les écailles qui les cachent. Il y a trois rangées de dents molaires à la mâchoire d'en haut, les dernières du rang interne sont rondes et les plus grandes. La mâchoire inférieure n'en a que deux rangées. Le devant de la bouche a quelques dents coniques, et derrière elles des dents en carde. Le palais est entièrement lisse. La dorsale répond à pou près au tiers do la pectorale : elle est basse. La caudale est fourchue, a lobes larges, et celui d'en bas est moins pointu que

1 Indiqué sur la planche de untre allas chrysophrys cyanopierel. Val. 
le supérieur. La pectorale est longue et aiguë; elle se termine à peu près sous le onzième rayon épinenx.

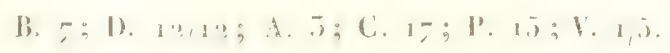

Les écailles sont grandes, osseuses et lisses. Il y on a cinquante le long de la ligne latérale, et quelques-unes sur la base de la caudale.

La ligne latérale est marquée par une série de petites tubulures; elle s'infléchit vers la queue. La couleur est. jaune pâle, avec du rougeâtre sur le devant de la face. Les nageoires sont bleuâtres.

La longueur de notre exemplaire est de $0^{\mathrm{m}}, 360$.

\section{GENRE GARANT.}

Le genre des Caranx est un des plus nombreux, nonstulement de la fantle des Scombere obles, mais de la classe entière des poissons.

L'espèce nouvelle que j'ai à décrire appartient à cetie uribu, qui a une seule fausse nageoire libre derrière la dorsale et l'anale. Elle a, comme tous ces Caranx, les formes allongées, la côte longue, et l'armure latérale te la queue cuirassée par de petits boucliers qui ne so montrent qu'au delà de la moitié du trone. Les pectorales sont plus longues que celles des autres. Ces formes lomnent à ce poisson une assez grande ressemblance avec nos Maquereaux, et que j’ai signalée en nommant eette espere. 
PANCHE VII.-- FIG.

\section{LE CARANA SCOHBWIN}

C"aranx scombrimus, Ial.

Le Caranx que j’ai à faire connaitre ici ressemble beaucoup a notre Marpuereau conmun par la forme asrondie de son eorps par la grandeur of la longrour die sa tète; mais il tient des Caranx par la petilesiede sa houchr et par les boudiers latéraus de la quenc. Il doit prendre place à côté des espèces qui ont une petite pinnule libre dessus et dessous la quene. C'est donc près des Caranx Alexandrimus, C. tela el autres espèces voisines qu'il faut le placer.

Le corps est rond, fusiforme. Sa hauteur est moindre que le sixième de la longueur totale; celle de la tête $\mathrm{y}$ est comprise quatre fois et quelque chose. L'œil est grand, éloigné du bout du museau d'une fois son diamètre, et contenu trois fois et un tiers dans la longuneur de la tête. Le sous-orbilaire est caverneux, les dents sont en velours ras. La première dorsale triangulaire touche a la seconde, qui est longue ef basse vers la fin. L'anale a la mème forme; la caudale pet bilobée ou peu profondément fourchue. La pinnule libre, supérieure ou inférieure, est élargie en palettes égales.

B. $7 ;$ D $99^{1}-1 / 27 ;$ A. $2 / 25 ; C, 17 ; 2,25 ;$ V. $1 / 5$.

Tout le corps est couvert de petites éculles très-

${ }^{1}$ N. B. Que le dessinateur at unblic la neuvieme et les pelits rayous rles nagenires. 
minces. Je compte jusqu'a quarante-deux petits bou. cliers, à partir de ceux de l'oxtrémité de la quene où ils sont encore visibles.

La ligne latérale n'a qu'une bien faible courbure.

La pectorale en faux atteint ì la fin de la dorsale. Le dos est vert foncé, devenant argenté sous le ventre. Les pectorales el les ventrales sont phus foncéen que les autres nageoires.qui sont tramsparentrs avee unn légère teinte verdàtre.

L'exemplaire a $0^{\mathrm{m}}, 4.00$ de long.

Cette espèce manque à l'Iehthyologric du Bragle, qui n’a d'ailleurs aucun autre Scombéroïle.

GENRE PRIONURE.

La famille des Teuthies se compose, comme on le sait, de trois groupes distincts : celui des Amphacanthes caractérisé par les deux épines de chaque ventrale, celui des Acanthures qui ont de chaque còté de la queue un aiguillon mobile que le poisson pent redresser à volonté, et relui des Nasons dont la queue. porte des boucliers osseux fixes, immobiles et sur lesquels il y a une carène relevée et tranchante.

La peau de tous ces poissons est dénuée d'écailles, même quand elle est chargée d'àpretís plus ou moins rudes au toucher.

Les dents, disposées sur un seul rang, sont lisses, coniques on quelquefois crénelées, el leurs caractères se combinent avec cenx que je viens de rappeler. J'ai établi des genres faciles à reconnaitre danc cette famille. 
Le's Jasons et leurs congénères sont tous originaires du grand Occan indien; je n'en connais encore aucunt espèce dans l'Atlantique.

Le Prionure que j'ai à faire connaître a l'aspect des poissons de ce genre, et comme j’ai publié ur Nason a trois hourliers, j'avais d'abord cru que le Teubie a trois ceussous, oriminaire des Galapagos, devait cure placé pris du Vison a trois boucliers d'Amboine, deja connu de Valenty et de Renard. Mais en étudiant cette nouvelle espèce avec plus de soin pour la décrire, je n’ai pas tardé à reconnaître que les dents avec leur rouronne dentelée placaient cette nouvello espece parmi les Prionures, et non pas parmi les Nasons qui ont leurs dents coniques et sans dentelures.

Elle prend alors un nouveau caractère d'intérèt, 'ar clle ajoute une troisc̀me espèce à un genre fondé sur une première due aux recherches de Péron. Elle confirme la justesse de nos vues, lorsque nous avons établi le grenre des Prionures. Nous voyons ce sonce répandu dans tout le grand Gcean, depuis la Nouvelle-Hollande jusqu'aux còtes du Japon, et en dernier lieu sur ce petit archipel des Galapagos rapproché du continent américain.

Cette famille des Teuthies doit être signalée aux recherches des voyageurs; elle est presque exclusivement confinée daus les mers de l'Océan indien; car à l'exception de quelques espèces d'Acanthures, et cn très-petit nombre, du grand bassin de l'Atlantique, toutes les autres si nombreuses viennent du grand

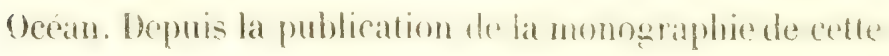


limille, donnce dans la grande histoire des ponsons, les collections nationales et étrangères se sont accrues d'un assez bon nombre d'espèces nouvelles.

\section{PLANCHE VII. - FIg. 2.}

LE PRIONURE A LARGE RAIE.

Prionurus laticlavius, Val. '.

Nous avons à ajouter aux Prionures déjà connus celui-ci, qui n'a pas de cornes avancées sur le front, ni même de tubérosité au devant des yeux.

Le profil, un peu soutenu et convexe entre les yeux, descend par une concavite profonde vers le museau qui fait une petite saillie par suite du rétrécissement de la face sur le derant des ycux. Au-dessus de ces organes la ligne monte par une courbe régulière pour former, avec la courbure un peu moins forte du ventre, l'ellipse du corps. La quene sort de cet ellipse. La hauteur fait le tiers de la longueur totale, jusqu'au centre du bord concave de la caudale. Le tronçon de la queue est trèsétroit, car sa hauteur ne fait que le cinquicme de celle du tronc. La longueur de la tète égale le cinquième de la longueur totale du corps. L'ocil est placé haut et en arrière sur la joue, éloigné ciu bout du museau de trois fois la longueur de son diamètre, et de la ligne du profil de moitié de ce diamètre. On ne voit rien de son orbitaire

* A tort sur la pl. VII : Naseus laticlavius. 
au devant de l'oeil; il est entièrement caché par la pear. Le bord du préoperenle se dessine par un ligue oblique assez haute; son angle est arrondi, et le bord inférieur est lćgèrement concave; l'operculc est faiblement strié et étroit, car il est deux fois plus haut que large, et sa largeurégale le diamètre de l'oeil. La bouche est peu fendue; le maxillaire est presque en entier reconvert par la peau qui entoure le sous-orbitaire el les côtés de la joue. L'intermaxillaire a peu de mobilité; il porte huit dents, ce qui en fait seize à la mâchoire supérieure. Elles sont élargies, comprimées, tranchantes, à émail ciselé, et à bord dentelé ou festomé de: quatre a cimy dentelures mousses et arrondies, et seulement sur le tranchant de la couronne et du côté externe ou postérieur. Le bord interne est lisse et sans aucune dentelure. La mâchoire infericure a seize on dix-huit dents comprinces of dont la couronne seule a le bord festonné. Le suscapu-

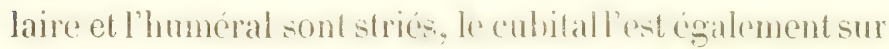
les bords do sa plaque qui est triangulaire, of chagrincé dans le milieu. La pectorale est obtuse et épaisse; elle est comprise cing fois et domie dins la longuem totale. Les ventrales ne sont pas très-longues; leur épine est forte et à peu près de moitié de la longueur du rayon.

La dorsale commence en arrière de l'aplomb de l'insertion de la pectorale; elle a huit rayons épineux, dont le promier est très-court. Les autres restent assez bas, car la hauteur de la nageoire ne mesure que le cinquième de la hauteur du corps. L'anale n'est pas plus élevée et de même forme; ello commene̊ sous les 
premiers rayons mous de la dorsale. La caudale a les tobes arroudis, whtur, an prolonges en pointe; le bord est concave.

$$
\text { D. } 8 / 28 ; A .7 / 25 ; \text { (. . 17; P. } 17 ; \mathrm{V}, 00 .
$$

Le corps n'a pas d'écailles, mais une sorte de cuir herissé de petites asperités tres-fines. De chaque còté de la queue, il y a trois boucliers ossenx. La couleur est jaune, aver une largo bande brune ou bistre éendue sur le côté. La tête est un peu plus foncée que le dos. La dorsale et l'anale ont les rayons jaunes et la membrane roussàtre; la caudale et la pectorale ont plus de jaune.

L'individu qui a fait le sujet de cette description a $0^{\mathrm{m}}, 390$ de longueur.

\section{GENRE LABRE.}

Les Labroïdes sont, comme on le sait, abondants et presque coralement répandus dans toutes les mers, depuis les deux pôles jusqu'à I'équateur. Les naturalistes explorateurs de l'archipel des Galapagos ne nous en ont fait connaitre que peu d'espèces. Je n'en vois qu'une seule décrite dans l'excellente publication des Poissons du Beagle. Elle appartient au genre des Cossyphes.

L'amiral Dupetit-Thouars a été plus heureux dans les recherches qu'il a fait faire. La petite collection que je fais connaitre en avait deux. L'une d'elles est aussi un Cossyphe.

L'autre est du genre Labre, róluit aux caractères que je lui ai assignés. Elle est nouvelle et doit prendre 
place a cóte du Labrus scrofia de l'Atlantique. J'indique' celte affinité en la nommant:

\section{PLANCHE VIII. - FIg. 1.}

\section{LE LABRE SANGLIER.}

Labrus aper, Val.

Ce grand ef beau Labre a la plus grande ressemblance avec le Labrus Scrofu, découvert dans le grand bassin de l'Atlantique, depuis les C'anaries et Madire, juscfu'aux ìles du cap Vert. La disposition des dents et la distribution des couleurs montrent le voisinage de ces espèces.

Ce poisson a le port et la forme régulière de nos Labres. Le profil est un peu soutenu sur le front jusqu'à la nuque. La longueur comprend trois fois et un quart la hauteur. La tête est un peu plus courte que la hauteur du tronc. L'oeil est ćloigné du bout du nuseau de deux fois le liamitre. Le sous-orbitaire est large et aché sous une peau épaisse. Le préopercule a le bord vertical lisse, sans dentelures; l'angle et le bord inférieur arrondi. Les dents de l'extrémitó et de l'angle de la mâchoire sont saillantes el comme des défenses; le reste de l'os porte des dents coniques égales, et le côté interne est couvert de petites granulations.

La dorsale et l'anale sont prolongées en pointes aisués; la caudale est peu échancrée à quelıues rayons externes prolongés en un lobe court; la pectorale est large et triangulaire.

B. $7 ; 0,12 / 12 ; 1,7 / 12 ; C ; 1,5 ; P, 18 ; V, 1 / 5$. 
Les ecailles sont de grandeur médiocre, jen compte soixante-deux rangées entre l'ouie et la caudale. Celle de l'opercule sont semblables à celles du trone, celles du préopercule sont heaucoup plus pelites; il y en a dix rangees sur un espace qui en contiendrait, à peine six de celles du tronc.

La couleur générale est un rouge brique plus ou moins vif, avec quelques ravures longitudinales qui se montrent par reflets. Une large tache jaune est sur chaque flanc an-(lessus de la pectorale. Toutes les nageoires sont blenâtres, la dorsale a une tache noire sur les quatre premiers rayons épineux, comme cela se trouve sur plusieurs autres Labres, ef notamment sur k" Labrus scrofa.

La ligne latérale n'est pas interrompue

La longueur de l'individu est de $0^{\mathrm{m}}, 4 \cdot 30$.

\section{DES COSSYPIIES.}

Ce genre n'est jusqu'à présent représenté dans I'Atlantique que par une seule espèce signalée déjà par Vargrave, mais dont les caracteres et les synonymies avaient été si mal élablis que j'ai eu beancoụ de peine à fixer la place de ce curieux Labroïde. Aujourd'hui que ce genre et ses espèces sont caractérisées et limitées, il m'a été facile d'ajouter à celle qui sont connues le nouveau Cossyphe que je vais décrire.

II a les formes du Cossyphe du Brésil, et en général de ces mers américaines; mais il rappelle par la disposition des couleurs nos espèces indiennes. 
Je ne conforme au désir qui m’a été exprimé par II. de Freminville, donateur de cette précieuse collection au Muséum, et dédiant celte belle en nouvelle espèce de Cossyphe à M. L'Éclancher.

J'ai dit plus haut que M. Jenyns avait publié un nouveau Cossyphe; il l'a nomme Cossuphus Darumi, en le dédiant au zoologiste habile ot distingue par ses helles observations sur les Coraux ef lis Zoophytes du srand Ucéan indien, comme je le fais pour l'oflicier du service de santé de l'expédition qui a formé cette collection.

PLANCHE VIII. - FIG. 2.

I,E COSSYPIIE DE I'ÉCLANCHER.

Cossyphus Eclancheri, Val.

Ce brillant poisson a l'aspect des autres Cossyphes; des ceailles reconreni la base des la dorsale, la portion molle de cette nageoire, l'anale et les ventrales prolongrées en pointes aiguës; de grandes écailles sur les opercules et font aisément reconnaitre ce poisson. La longueur de la tête égale à la hauteur du corps fait le quart de la longueur totale. Le front est assez sontenu entre les yeux, le museau est aigu; l'œil de grandenr médiocre a un diamctre qui ne fait que le sixième dé la longueur de la tête, et a une fois ce diamètre au-dessous de la ligne du profil. Le préopercule est couvert de petites écailles, mais celles de l'opercule ressemblent presque à celles du corps par leur srandeur. 
La pectorale est large et insérée sous l'aplomb de l'angle de l'opercule. Le premier rayon de la corsale est un peu plus reculé; sa hauteur égale les deux tiers de la dernière épine, et n'est pas le tiers du rayon le plus long de la dorsale. La caudale a le bord coupé carré. ment, et ses angles arrondis n'ont aucun espace de prolongement. Les ventrales égalent en longueur près des quatre cinguiemes de la hauteur correspondante du trone.

$$
\text { D. } 12 / 10 ; \text { A. } 5 / 12 ; \text { C. } 12 ; \text { P. } 18 ; \text { V. } 1 / 5 \text {. }
$$

Les écailles sont minces et assez grandes, j'en trouve trente-deux rangées entre l'ouie et la caudale. La ligne latérale est tracée parallèlement an dos par une suite de tubaclures, sous l'extrémité postérieure se redresse un peu vers la dorsale. Tout le corps est peint d'un beau rouge rermillon, avec de grambes maculatures noires, étendues sur les six premiers rayons de la dorsale, et descendant sur le dos, puis couvrant toute la base de la dorsale et te dos de la queue sams atteindre la caudale. Ces taches noires remontent sur toute la portion molle de la dorsale en laissant sur les deux bords un liséré rouge vif.

Ce poisson est grand comme nos Tourds ordinaires (Labrus Turdus, Nob.). Il a $0^{\mathrm{m}}, 4.39$ de longueur.

\section{GENRE TURBOT.}

$$
\text { Passer, Cuv. }
$$

Le nom de Rhombus, sous lequel M. Cuvier a ctabli, aver rason, la coupe génerique des Turbots de la fa- 
mulle des Pleuronectes avait éte déjà emplose par Lacepède pour un poisson voisin des Stromatées, et que Linné a fait comnaitre sous le nom de Chetodon Alepidotus. Il lo tenait de Garden. En faisant la monographie de ce genre (Hist. nat. Poissons, tome IX, p. 4.00), j'ai dit que je reprenais les dénominations consacrees par Lacépède, et que M. Cuvier avait eu tort de ne pas accepter diuss le Rigme animul. N'ayant pas recomnu le grenre ctabli par iacépede, il n'en avait pas moins hien recherché et déterminé le poisson de Garden, et crovant qu'il fallait en faire un genre distinct, M. Cuvier le désigna par un nom nouveau, celui de Peprilus. Lorsqu'il en vint a parler, dins la famille de.. Pleuronectes, du geme des Turbots, il trouvar la sramble espere de nos mers, désignée par Linné sous le nom de Pl. Rhombus. C'est bien, en effet, le Rhombus des naturalistes grees et latins; il faut cependant remarcjuer qu'Athénée et Naurate, cité par le premier de ces deux savants ont

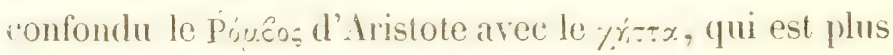
spécialement notre Barbue. Faisant aver raison un getnre de ces Pleuroncetes à dorsale avance jusque vers le bord de la mâchoire supérieure, l'auteur du Règne animal désigna sa nouvelle coupe gencingue par l'épithete que Linné avait empruntée aux anciens. Mais si M. Cuvier avait d'abord cherché à reconnaître le genre désigné par Lacépède, sous le nom de Rhombus, il n'aurait pas employé, quoiqu'à regret sans doute, cette dénomination pour le nouveau genre de la famille des Pleusonectes. Jusqu'au travail que j'ai donné sur la famille sles Stromatées, les tehthyologistes ont admis le nom 
Ae Rnumbs pour designer le genre de Pleuronectes établi par Cuvier. J'avoue qu'après avoir reconnu le genre de Lacépède qui était resté presque ignoré, tandis que celui de Cuvier avait été accepté; j’ai eu beaucoup de peine à me déterminer à rahlir les noms suivant leur chronologie. Je sentais bien, et je sens encore que ce changement de nom va faire une petite perturbation dans la nomenclature; et la langue des zoologistes el surtout celle des Ichthyologistes est dijà assez surchargee de noms inutiles, faisant double emploi ou confusion, porir ne pas y ajouter encore en voulant rectifier. D'un autre còté, j'ai cru qu'il était de tonte justice, surtont dans un ouvrage, comme celui auquel II. Cuvier m'a fait consacrer une partie de ma vie, en recherches littéraires et synonymiques, la dénomination qu'un homme aussi illustre que Lacépède avait donnée à un genre parfaitement établi. Ce grand zoologgiste s'est servi du nom de Psettis cmployé deja dans les manuscrits de Commerson pour un genre bien déterminé de la famille des Squamipennes. Je n'aurais pas hésité à donner au genre des Turbots le nom latin tiré de la dénomination de la Barlue. Mais je ne pouvais pas le faire. J'ai pris alors dans les noms latins imposés aux Pleuronectes un de ceux que nos prédécesseurs n'avaient encore employé; celui de Passer, l'un des plus connus, et que par lequel tant d'auteurs ont designe les Pleuronectes, m’a paru convenable. Quand j’aurai publié, soit en entier, soit par extrait, la Monographie des Pleuronectes dans la secoude séric de 
l'Histoire des Poissons, josprere établir définitrement cette nouvelle dénomination, et qu'elle sera acceptée par les zoologistes.

D’ailleurs je ne serais pas éloigné de croire que le poisson que je décris ici sous le nom de Passer. Marchionessarum ne constiluera aver le Pl. Podas, et quelques especes voisines, un grenre distinct du Turbot ef de la Barbue des mers d'Luropre; mats ces coupes ne peuvent ètre faites qu'a lat suited'un examen détaillé et critique de toutes les espèces de cette grande famille, et ce n'est pas ici le lieu d'établir cette discussion.

\section{PLANCHE IX.}

\section{LE TURBOT DES ILES MARQQUISES.}

Passer Marchionessarum, Val.

L'espèce nouvelle din grand Océan a de la ressemblance avec les espèces de l'Atlantique, et même avec le Plenronectes Podas de la Méditerrannée. Elle tient cette aflinité du grand écartement des yeux de la saillie des crètes surcilières et de l'épine relevée qui termine la maxillaire.

Ce Turbot a le corps allongé; la largeur des flanes fait le tiers de la longueur totale. La tête y est comprise quatre fois et trois quarts. Le profil est un peu sinueux entre les deux yeux; l'œil gauche est inférieur, répond à l'angle supérieur de la bouche, le droit ou le supérieur est éloigné du premier de deux fois le diamètre; a l'intervalle un peu crenx qui les sépare est 
lisse, le préopercule a son bord vertical droit, l'autre descend tres-obliquenent vers l'angle de ha màchoire: il est tout couvert d'écailles comme les autres pièces de l'appareil operculaire. Les dents sont en cardes trèsfines. Il y en a aussi sur le chevron du vomer. Outre lá grande espèce du maxillaire, il y en a une seconde plus courte et cannelée le long de l'os. La dorsale est étendue depuis le devant de l'œil jusqu'auprès de la queue. L'anale commence à l'aplomb de l'épaule, la caudale est arrondie. La pectorale gauche est longue, et les trois premiers rayons sont prolongés en filaments déliés; leur longueur entière portée sur le corps y est égale à la hauteur du tronc, ou est comprise trois fois el trois quarts dans la longueur totale.

La longueur de la pectorale droite est du tiers de la gauche.

$$
\text { B. } 7 \text {; D } 9^{n} ; A .70 ; \text { C. } 16 ; \text { P. } 11 ; \text { V. } 15 .
$$

II y a environ cent rangées de petites écailles le long des flanes; elles sont toutes marquies de stries concentriques ef grenues. La ligne latcrale est fortement courbée à son origine au-dessus de l'insertion de la pectorale, elle se rend ensuite droite à la quene par le milieu du corps.

La couleur est brune du còté gauche, et blanche de l'autre côté.

Ce poisson m'a élé indiqué comme provenant des îles Marquises, il est long de $0^{\mathrm{m}} \cdot 4.00$. Il serait le senl de cetle collection qui fuit étranger aux Galapagos. 
GLNRE MURENE.

II. Darwin a trouvé aux Galapagos un Gobiésoce que les navigateurs sur la Vémus n'y ont pas observé. Ce poisson, décrit par M. Jenyns, est devenu le Gobiesox precilophlihatmus. Je citre cetto jolic espece pour completer ici la revue des Poissons déjà connus de l'archipel.

La famille des Anguilliformes a fourni au contraire des espires aux deux expeditions. Elles appartiennent toutes deux au genre Murène.

Les Ichthyologistes ont fous sonti la nócessité de subdiviser le grand genre Mun wa de Limne. Déjà Thunberğ, sous le nom de Murcena, Bloch, sous celui de Gymnothorax, Lacépede, sous celui de Muranophis, ont réuni les espèces sans pectorales, pour les séparer des Anwuilles qui ont les nageoires thoraciques plus on moins developpés. L’on trouvera dans l'analyse de la famille des Anguilliformes que j'ai raison d'employer une expression douteuse sur la valeur du caractère fourni par la présence ou l'absence de la pectorale; car ces nageoires diminuent par degrés insensibles. L'étude des dents foumira des caractires bien plus positifs; elle démontrera que les premieres modiilicalions apportées par l'autcur du Regne animal au quenre Murena de Linne. n'étaient que l'ébauche d'un travail plus considérable, qui doit être précédé de la description et de la dissection des nombreuses especes de cette famille très-nombreuse et répandue dans toutes les mers.

1I. Darwin a pris à l'ile Chatam et à l'île Charles les 
deux exemplaires du Muranophis nomme par M. Jenyns Muranophis lentiginosa. Il n'en a pas publié cle figure. La description que j'ai comparée à mon poisson ne lui convient pas assez pour que je le regarile comme de la même espèce.

Celle que je décris ici appartient au groupe des Murxnophis, dont la gueule est armée de dents pointues et tranchantes sur un seul rang, et qui ont les nageoires impaires assez élevées pour en énumérer facilement le nombre des rayons. Il me parait prohable qu'elle restera daus le genre qui sera plus spécialement désigné par le nom générique sous lequel je vais la faire connaître.

PLANCHE X. - Fig. 1.

LA MURÉNE MARBREE.

Murenophis marmoreus, Val.

Cette Murène élégante par ses couleurs, appartient à cette division des espèces à dents triangulaires, pointues, tranchantes sur les côtés, sans dentelures ni crochets. Le vomer en porte aussi une séric longitudinale; mais les palatins sont lisses. Les veux sont au milieu de la longneur de la fente de la gueule, l'occiput est peu soutenu, les narines antérieures sont tubuleuses, l'ouverture postérieure est une fonte longitudinale sous l'œil. Il n'y a comme dans les espèces de ce genre aucunes nageoires paires, le nombre des rayons des trois 
autres rémis, et de quatre cents et quelques, car j'en compte

$$
\text { D. } 164 ; \text { A. } 127 ; \text { C. } 12 \text {. }
$$

Tout le corps est marbré de brum sur un fond jaune.

Longueur $0^{\mathrm{m}}, 530$.

\section{GENRE TETPODON.}

Deux espèces du genre Tétrodon ont été observées aux Galapagos. L'une a été rapportée par M. l'amiral Dupetit-Thouars; mais l'exenplaire, en assez mauvais état, n'a pu être figuré; ct je n'en aurais pas même parlé, si MI. de Freminville n'avait joint à l'envoi de cette collection le dessin de cette espèce. Il m'a servi à reconnaitre le Tetrodon angusticeps de M. Jenyns.

La scconde ne m'est connue que par la description du Beagle : c'est le T'rodon annulatus. C'est une des espèces dont la peau est couverte de fines épines.

\section{I.E TETRODON A TETE ETROITE.}

Tetrodon angusticeps, Jenyns, Foy. du Beagle, pl. 28.

Celui-ci est du petit nombre des Tétrodons à peau lisse et sans ćpines. Sous re rapport, elle tieut plus des Tétrodons de l'Alantique que de ceux de la mer dos Indes.

La tête est oblongue et ćtroite. Les yeux sont trèsrapprochés; l'intervalle qui les sépare égale leur diamètre. Le dos est droit, le ventre assez renflé, et l'animal peut se gonfler beaucoup. I a dorsale cst pe- 
tite et répond à l'anale, qui est aussi étroile. La caudale est coupcée carrément. La ligne latérale est infléchie sur le corps et rameuse sur la tête; une branche fait une grande anse sur la narine; deux autres vont, en travers, se perdre sur la nuque et sur le commencement du dos, sous la première de celle-ci, un rameau descend sur la joue.

Le dessin, coloré par M. de Freminville, peint le dos en brun, ainsi qu'une large bande qui court sur le bas des côtés. Une autre bande de couleur jaune sépare le brun du dos de celui des côtés. Tout le dessous du corps est également jaune, ainsi que les dorsales, l'anale et les pectorales. La caudale est brune. Une lachefoncere, presque noire, est a la base de la pectorale.

M. Jenyns indique des teintes un peu différentes. Suivant ce zoologiste, le dos serait verdâtre, un peu rembruni; la base de la pectorale et la dorsale seraient noires. Na-t-il pas dit, par inadvertance, dorsale pour caudale? I'ailleurs .I. Jenyns reconnaît que les couleurs du poisson ont été altérés par l'action de l'alcool.

Malgré ces légères différences, je ne doute pas de l'identitespécifique des poissons recueillis par M. Darwin, sur le Beagle, et par M. L'Éclancher, sur la Vénus.

\section{GENRE CESTRACION.}

Le genre des Cestracions est encore une des excellentes créations de Cuvier. Elle a fixé la place d'un Squale des mers de la Nouvelle-Hollande, qui paraissait tout a fait étranger dans cette grande famille, à cause de la

ICUTHYOLOGIE. 
-ingularifi de sa dentition. Los caracteres ef les formes de ses dents sont, en eflet, tellement différentes de celles des autres Squales, que l'on ne peut hésiter sur la fixité que donne l'étude de ces dents. Elle a un autre avantage, e'est de faire connaître d'une manière plus précise les rapports des Hybodes et autres Squalides connus par lcurs dents fossiles et communes dans la période crayeuse.

\section{PLANCIIE X. - Fig..}

\section{IE CESTRACION PANTHERIN. \\ Cestracion pantherinus, Val.}

Ce poisson de la famille des Squales a le port d'une Monssette : lo dessus de la tête est plus crenx, mais il l'est beancoup moins que celui du Cestracion Phillipi, qui habite les eaux de la Nouvelle-IIollande. Ce peu de profondeur de la gouttière occinitale dépend du pou de redressement des arcades surcilières.

La première dorsale l'éleve au tiors du corps, ot la seconde au milieu de l'intervalle entre l'épine de cette première dorsale of la caudale. Les espèces sont grosses, un peu renfíes, et n'atteignent qu'à moitié de la hill!tenr de la nageoire. L'angle de ces nageoires est arrondi, la membrane est trapézoïdale. L'anale est étroite arrondie. La caudale a le lobe supérieur tronqué, large et à angles mousses; lo lobe inférieur est triangulaire.

La pectorale est insérée au milicu de l'espace entre 
le bout du muscau et la ventrale, et coiles-ci sont aux deux cinquièmes du corps. Ces nageoires paircs sont assez larges, à bords arrondis.

La troisième des cinq fentes branchiales est à l'angle de la pectorale; je vois sous l'œil un petit avant.

La bouche n'est pas très-large, elle porte en avant cinq à six rangs de potites dents aiguës, ayant à la base deux petits talons épineux, puis viennent sur les cotés des màchoires six rangées de molaires arrondies et carénées sur le milieu.

Toute la peau est couverte d'aspérités mousses it l'extrémité du museau, sur les nageoires et mòme sur le palais; mais sur le tronc et au delà, ce sont des tubercules à trois pointes aiguës, les deux latérales comme de petits talons, et implantées en quinconces serrés, etc.

La couleur est un gris roussâtre, avec de grosses laches noires arrondies éparses sur les corps et sur les nageoires.

L'individu est un mìle, ses appendices sexuels sont gros, coniques monsses, et dépassant la ventrale; ils sont presque aussi longs qu'elle.

La longueur est de $0^{\mathrm{m}}, 17 \%$. 



\section{TABLE DES HATIPBES}

M A M M I F E RS.

Praeses

Introluction $\ldots \ldots \ldots \ldots \ldots \ldots \ldots \ldots \ldots \ldots \ldots \ldots \ldots \ldots \ldots \ldots \ldots$

Première section. - Primates...................

Denxième section. - Carnassiers................... 110

Troisième section. - Rongeurs ..................... 154

Explication des planches de Mammifères................ 169

Index des espèces de Mammifères décrites ou indiquées, nt des noms génériques et spécifiques cités............... 175

OISEA UX.

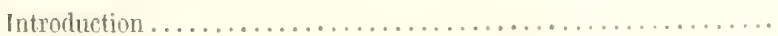

Index des espèces l'Oiseaux décrites ou indiquées, of des noms génériques et spécifiques cités.

Z EPTLES.

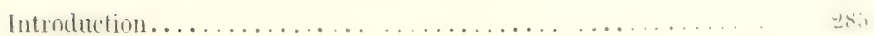

Proctotrète du Chili............ Pl. 1, fig. 1 et $2 . . . \ldots$. .

- intermédiaire........ Pl. 2, fig. 1......... gو

- ventre bletr......... Pl. 2, fig. 2......... gy

- it taches noires....... Pl. 3, fig. 1......... 293

- de Wiegmann........ Pl. 3. lis. 2... .... .. 245 
ICHTHYOLOGIE

Pages.

Introduction ............................... 297

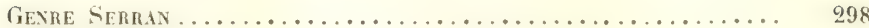

I, Serran Psittaciu............ Pl. : tig. 1.......... 299

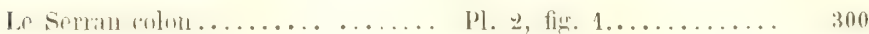

Gexne Diacofe................................. 302

La Diacope verte ............... Pl. 1, fig. 2.......... 303

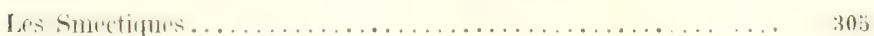

Smectique aux deux couleurs....... Pl. 2, fig. 2.......... 307

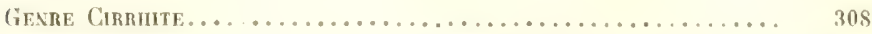

Cimbite rivulé ................ Pl. 3, fig. 1.......... 309

Gente Scorpène.................................. 312

La scorpène fardée............. Pl. 3. tig. Q.......... 313

Gienre Myripristis............................... 315

Le Myripristis anx màchoires lisses,

ou Myripristis Leignathe........ Pl. 4, fig. 1......... 316

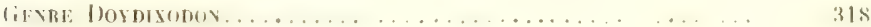

le Doydixodon de Freminville.... Pl. 5 .............. 39.3

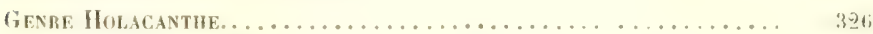

L'Holacanthe moineau.......... Pl. f..............

Genre Daurade................................. 329

La Daurade cyanoptère......... P. 4, fig. $4 \ldots . . . . . .330$

Gexne Caranx,.................................. 331

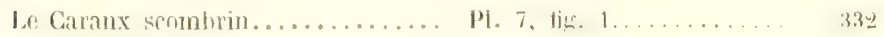

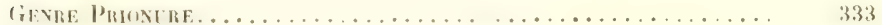

Le prionure a large raie.......... Pl. 7, fic. $2 \ldots . . . . . .$. . $33 ;$

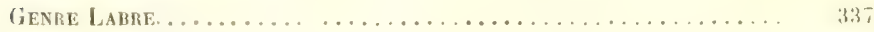

Le Labre sanglier............. Pl. 8, tig. 1......... 33s

Drs Cossyphes............................... 339

Le Cossyphe de l'Éclancher........ Pl. 8, fig. 2......... 340

Gente T'trbot................................. 341 
Le Turbot des lles Marquises..... 1'l. $9 \ldots \ldots \ldots \ldots \ldots \ldots$.......

Genre Munène ............................... $3 ; 6$

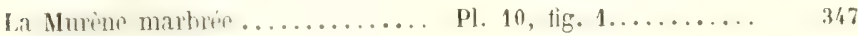

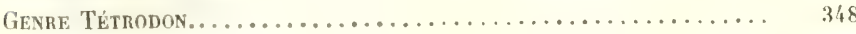

Le Tétrodon à tète étroite......................... $34 \mathrm{~s}$

Genre Cestracion............................. 349

L.e Cestracion panthérin......... Pl. 10, fig. 2........ 3,

EIX DE I,A TABLE DES MATIËRES. 







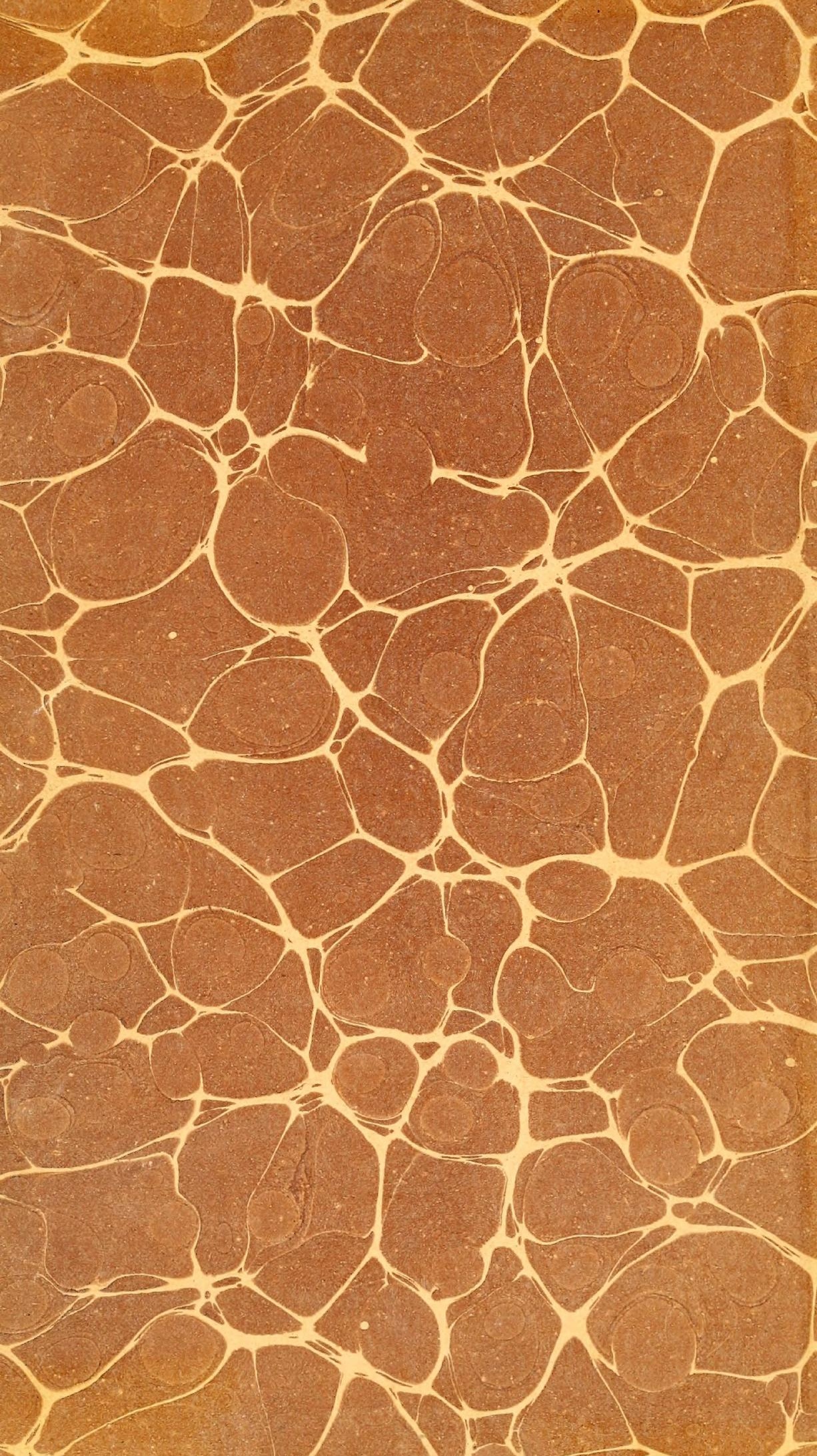


